Florida International University

FIU Digital Commons

6-6-2019

\title{
Affective States and Work Attitudes Linking Abusive Supervision to Employee Performance and the Impact of Ethical Climate on Abusive Supervision and Work Attitudes
}

Armando Falcon

Florida International University, afalc008@fiu.edu

Follow this and additional works at: https://digitalcommons.fiu.edu/etd

Part of the Industrial and Organizational Psychology Commons

\section{Recommended Citation}

Falcon, Armando, "Affective States and Work Attitudes Linking Abusive Supervision to Employee Performance and the Impact of Ethical Climate on Abusive Supervision and Work Attitudes" (2019). FIU Electronic Theses and Dissertations. 4263.

https://digitalcommons.fiu.edu/etd/4263

This work is brought to you for free and open access by the University Graduate School at FIU Digital Commons. It has been accepted for inclusion in FIU Electronic Theses and Dissertations by an authorized administrator of FIU Digital Commons. For more information, please contact dcc@fiu.edu. 


\title{
FLORIDA INTERNATIONAL UNIVERSITY \\ Miami, Florida
}

\begin{abstract}
AFFECTIVE STATES AND WORK ATTITUDES LINKING ABUSIVE
SUPERVISION TO EMPLOYEE PERFORMANCE AND THE IMPACT OF

ETHICAL CLIMATE ON ABUSIVE SUPERVISION AND WORK ATTITUDES
\end{abstract}

\author{
A dissertation submitted in partial fulfillment of \\ the requirements for the degree of \\ DOCTOR OF PHILOSOPHY \\ in \\ PSYCHOLOGY
}

by

Armando Falcon

2019 
To: Dean Michael R. Heithaus

College of Arts, Sciences and Education

This dissertation, written by Armando Falcon, and entitled Affective States and Work Attitudes Linking Abusive Supervision to Employee Performance and the Impact of Ethical Climate on Abusive Supervision and Work Attitudes, having been approved in respect to style and intellectual content, is referred to you for judgment.

We have read this dissertation and recommend that it be approved.

Valentina Bruk-Lee

Asia Eaton

Hock-peng Sin

Chockalingam Viswesvaran, Major Professor

Date of Defense: June 06, 2019

The dissertation of Armando Falcon is approved.

Dean Michael R. Heithaus

College of Arts, Sciences and Education

Andrés G. Gil

Vice President for Research and Economic Development and Dean of the University Graduate School

Florida International University, 2019 
(C) Copyright 2019 by Armando Falcon

All rights reserved. 


\section{DEDICATION}

To my loving parents, Olga and Armando. 


\section{ACKNOWLEDGMENTS}

I would like to acknowledge Dr. Valentina Bruk-Lee, for introducing me to the field of industrial/organizational psychology and for her continued guidance and support. I would also like to thank Dr. Chockalingam Viswesvaran for his enduring patience with me and for providing consistent technical support and advice throughout my studies. Finally, I thank the Florida Education Fund for their financial support—via the McKnight Doctoral Fellowship — during the initial three years of my graduate studies. 


\begin{abstract}
OF THE DISSERTATION
AFFECTIVE STATES AND WORK ATTITUDES LINKING ABUSIVE

SUPERVISION TO EMPLOYEE PERFORMANCE AND THE IMPACT OF

ETHICAL CLIMATE ON ABUSIVE SUPERVISION AND WORK ATTITUDES

by
\end{abstract}

Armando Falcon

Florida International University, 2019

Miami, Florida

Professor Chockalingam Viswesvaran, Major Professor

Prior research indicates that abusive supervision (AS, Tepper, 2000) is associated with employee job performance. On the other hand, ethical organizational climate (EOC; Victor \& Cullen, 1988) has yet to be investigated in the context of AS. The objective of the present study is to evaluate, within a general sample of US employees, (1) the relative strength of specific indirect effects of AS on performance via positive (PA) and negative (NA) affect, and via work-related attitudes (Leader-member exchange, LMX; Interactional justice, IJ; Perceived organizational support, POS; Affective organizational commitment, AOC), and (2) the relationship between EOC, AS, and the same work-related attitudes. It was expected that the indirect effects of AS on performance would be stronger via affect (PA, NA) than via attitudes referencing the supervisor (LMX, IJ), which would in turn be stronger than the indirect effects via attitudes referencing the organization (AOC, POS). Moreover, EOC was expected to show a negative association with AS, a positive association with each work-related attitude, and to moderate the effects of AS on the workrelated attitudes. Data was collected using web-based questionnaires. Employees 
completed attitude measures and rated how frequently they experienced AS and affective states in the prior month. Coworkers rated job performance. Analyses take a nonparametric approach due to departures from multivariate normality. Indirect effects and pairwise contrasts between specific indirect effects were evaluated using bootstrap confidence intervals. Hypotheses involving moderation were analyzed using moderated multiple regression. Mediation analyses generally confirm prior findings when indirect effects were analyzed separately. When modeled in parallel however, only the indirect effects via NA and AOC are significant. Pairwise contrast generally failed to show significant differences between specific indirect effects. Consistent with predictions, EOC was negatively associated with AS and positively associated with each work-related attitude. Interestingly, EOC also moderated the effects of AS on work-related attitudes, albeit in a direction inconsistent with predictions. Implications for future research are discussed. 


\section{TABLE OF CONTENTS}

CHAPTER

PAGE

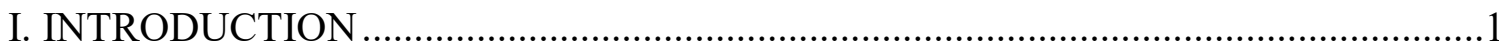

Organizational/Workplace Aggression ..............................................................

Subordinate Job Performance ...........................................................................

Mechanisms Linking Abusive Supervision to Subordinate Job

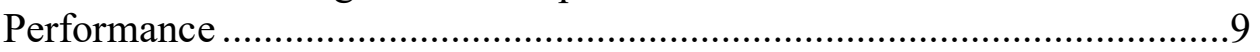

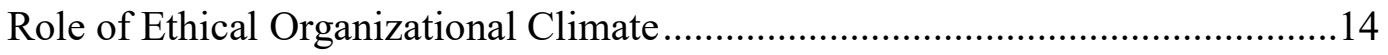

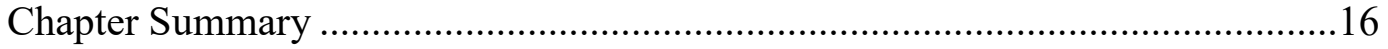

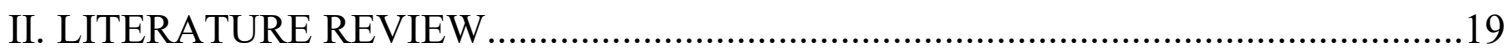

Abusive Supervision: Subordinate-targeted Aggression .....................................19

Defining Abusive Supervision.................................................................20

Conceptual and Methodological Issues ..................................................21

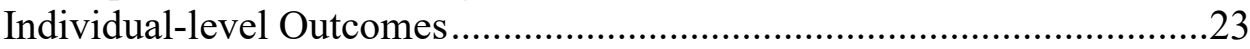

Abusive Supervision and Subordinate Job Performance........................................24

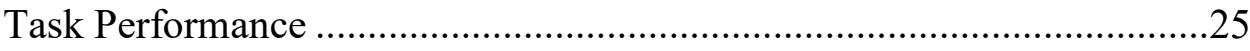

Organizational Citizenship Behaviors ...................................................26

Counterproductive Work Behaviors …………………............................27

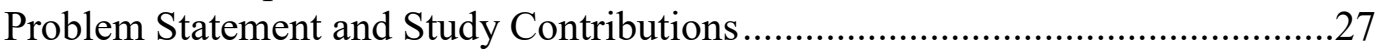

State Affect versus Attitudes .....................................................................31

Supervisor- versus Organization-referenced Attitudes.................................33

Affective Mechanisms Linking AS to Job Performance …………….....................35

Abusive Supervision and State Affect.....................................................36

Supervisor-referenced Attitudes Linking AS to Job Performance …………...........39

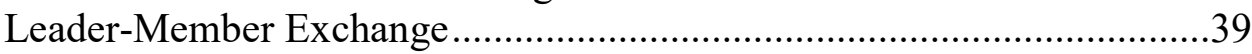

Interactional Justice ……………….................................................... 41

Organization-referenced Attitudes Linking AS to Job Performance .....................42

Perceived Organizational Support .........................................................42

Affective Organizational Commitment.....................................................43

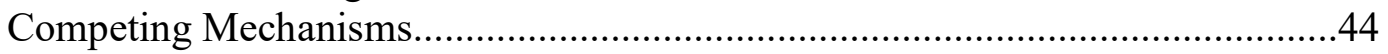

Ethical Organizational Climate ........................................................................46

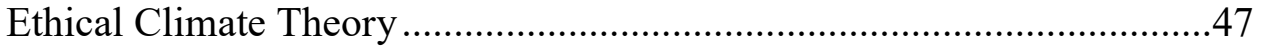

Moderating Role of PEC...................................................................5

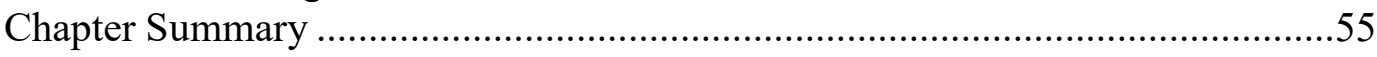

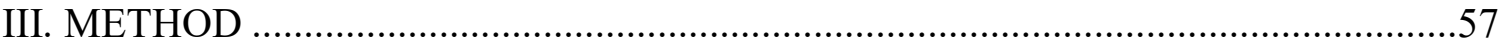

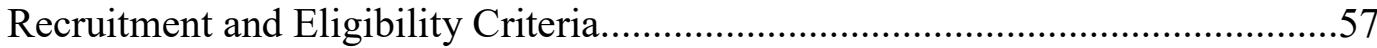

Participants and Procedure........................................................................58

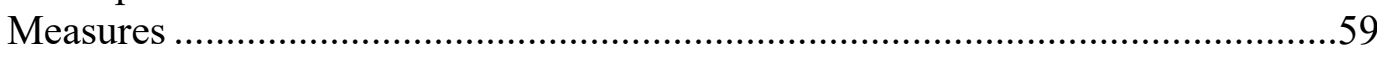

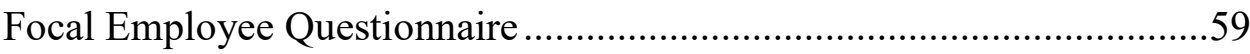

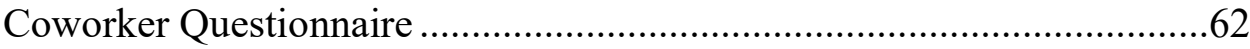

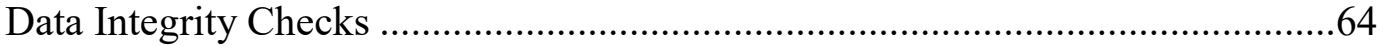




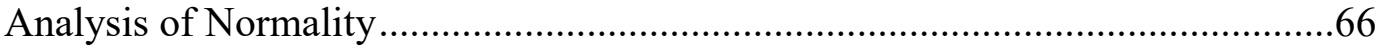

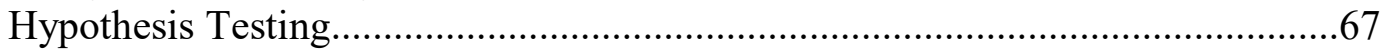

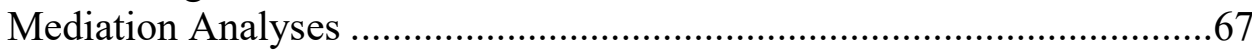

Moderated Multiple Regression..............................................................74

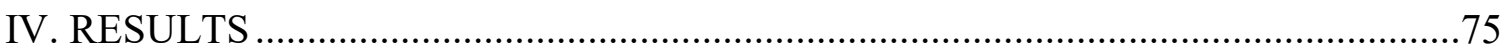

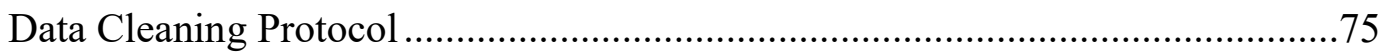

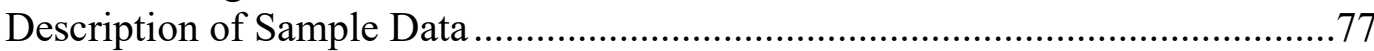

Matched-only Dataset .............................................................................

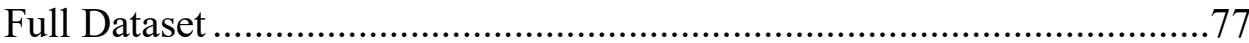

Comparison of Matched and Unmatched Samples ...............................................78

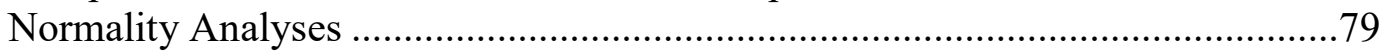

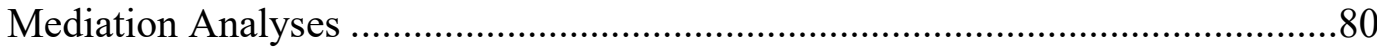

Mediating Effect of Positive Affect.......................................................8

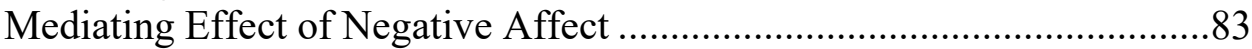

Mediating Effect of Leader-Member Exchange .........................................85

Mediating Effect of Interactional Justice ..................................................8 87

Mediating Effect of Perceived Organizational Support..............................89

Mediating Effect of Affective Organizational Commitment ......................91

Pairwise Contrasts Between Specific Indirect Effects................................93

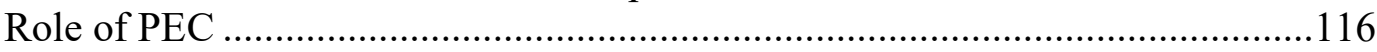

Correlational Analyses .........................................................................116

Confirmatory Factor Analyses ..........................................................117

Moderated Multiple Regression..........................................................118

Structural Equation Modeling ...............................................................121

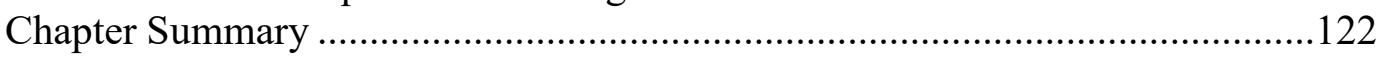

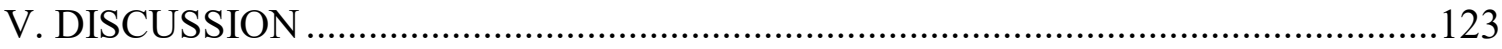

Background and Purpose ……………………………….............................123

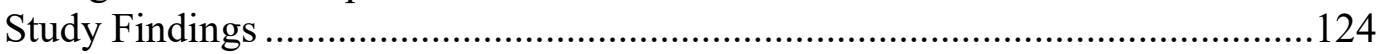

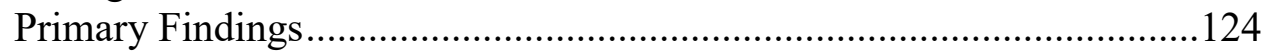

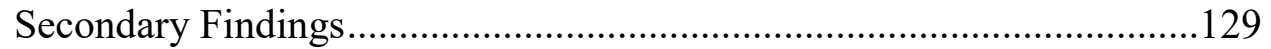

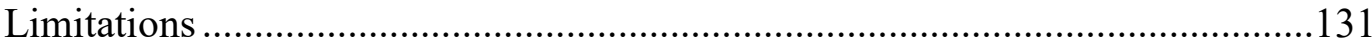

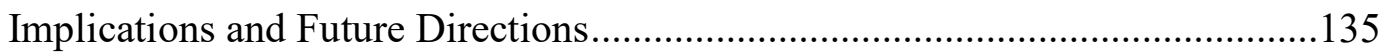

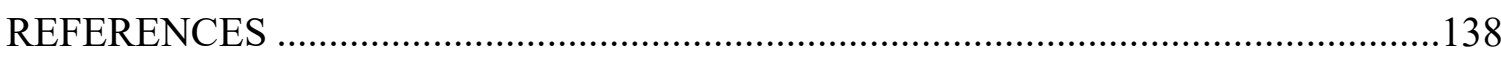

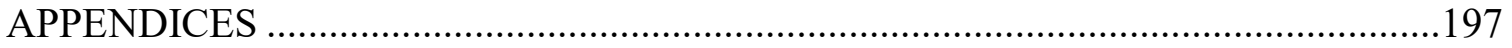

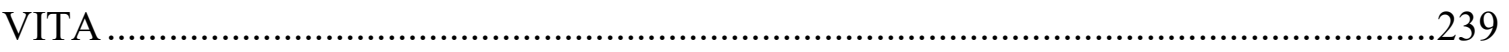




\section{LIST OF TABLES}

TABLE

PAGE

Table 1. Meta-analytic relationships between abusive supervision and study variables 156

Table 2. Corrected correlations between mediators and job performance variables 157

Table 3a. Data cleaning efforts for focal employee participants ................................158

Table 3b. Data cleaning efforts for coworker participants ......................................159

Table 4. Variable distributions across matched and unmatched groups (continuous variables) 160

Table 5. Demographic characteristics across study datasets and US labor force .161

Table 6a. Pearson zero-order correlations and internal reliability estimates (full dataset; $\mathrm{N}=2738$ ). 162

Table 6b. Pearson zero-order correlations and internal reliability estimates (matched-only dataset; $\mathrm{N}=281$ ) 163

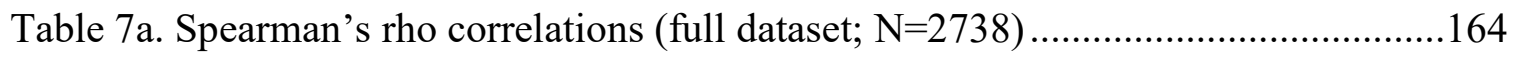

Table 7b. Spearman's rho correlations (matched-only group; $\mathrm{N}=281$ ) ..........................165

Table 8a. Univariate normality tests for all study variables (full dataset) ......................166

Table $8 \mathrm{~b}$. Univariate normality tests for all study variables (matched-only dataset) ......169

Table 9a. Direct and Indirect Effects of AS on Task Performance via PA

(Hypothesis 1a)

Table 9b. Direct and Indirect Effects of AS on OCBO via PA (Hypothesis 1b)............172

Table 9c. Direct and Indirect Effects of AS on OCBI via PA (Hypothesis 1c)..............172

Table 9d. Direct and Indirect Effects of AS on CWBO via PA (Hypothesis 1d)............173

Table 9e. Direct and Indirect Effects of AS on CWBI via PA (Hypothesis 1e).............173

Table 10a. Direct and Indirect Effects of AS on Task Performance via NA (Hypothesis 2a) 
Table 10b. Direct and Indirect Effects of AS on OCBO via NA (Hypothesis 2b) ..........174

Table 10c. Direct and Indirect Effects of AS on OCBI via NA (Hypothesis 2c) ............174

Table 10d. Direct and Indirect Effects of AS on CWBO via NA (Hypothesis 2d) .........174

Table 10e. Direct and Indirect Effects of AS on CWBI via NA (Hypothesis 2e) ...........175

Table 11a. Direct and Indirect Effects of AS on Task Performance via LMX (Hypothesis 3a)

Table 11b. Direct and Indirect Effects of AS on OCBO via LMX (Hypothesis 3b).......175

Table 11c. Direct and Indirect Effects of AS on OCBI via LMX (Hypothesis 3c).........176

Table 11d. Direct and Indirect Effects of AS on CWBO via LMX (Hypothesis 3d) ......176

Table 11e. Direct and Indirect Effects of AS on CWBI via LMX (Hypothesis 3e) ........176

Table 12a. Direct and Indirect Effects of AS on Task Performance via IJ (Hypothesis 4a)

Table 12b. Direct and Indirect Effects of AS on OCBO via IJ (Hypothesis 4b) .............177

Table 12c. Direct and Indirect Effects of AS on OCBI via IJ (Hypothesis 4c) ................177

Table 12d. Direct and Indirect Effects of AS on CWBO via IJ (Hypothesis 4d) ............178

Table 12e. Direct and Indirect Effects of AS on CWBI via IJ (Hypothesis 4e) ..............178

Table 13a. Direct and Indirect Effects of AS on Task Performance via POS

(Hypothesis 5a) .178

Table 13b. Direct and Indirect Effects of AS on OCBO via POS (Hypothesis 5b) ........179

Table 13c. Direct and Indirect Effects of AS on OCBI via POS (Hypothesis 5c) ..........179

Table 13d. Direct and Indirect Effects of AS on CWBO via POS (Hypothesis 5d)........179

Table 13e. Direct and Indirect Effects of AS on CWBI via POS (Hypothesis 5e)..........180

Table 14a. Direct and Indirect Effects of AS on Task Performance via AOC (Hypothesis 6a) 
Table 14b. Direct and Indirect Effects of AS on OCBO via AOC (Hypothesis 6b) .......180

Table 14c. Direct and Indirect Effects of AS on OCBI via AOC (Hypothesis 6c) .........181

Table 14d. Direct and Indirect Effects of AS on CWBO via AOC (Hypothesis 6d) ......181

Table 14e. Direct and Indirect Effects of AS on CWBI via AOC (Hypothesis 6e) ........181

Table 15a. Specific indirect effects of AS on task performance.......................................182

Table 15b. Specific indirect effects of AS on OCBO ………........................................182

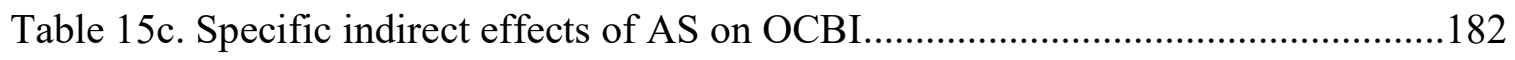

Table 15d. Specific indirect effects of AS on CWBO ..................................................183

Table 15e. Specific indirect effects of AS on CWBI...................................................183

Table 16. Contrasts between specific indirect effects associated with PA and AOC (Hypotheses 7a-7e)

Table 17. Contrasts between specific indirect effects associated with PA and POS (Hypotheses 8a-8e) 184

Table 18. Contrasts between specific indirect effects associated with PA and LMX (Hypotheses 9a-9e) 184

Table 19. Contrasts between specific indirect effects associated with PA and IJ (Hypotheses 10a-10e)

Table 20. Contrasts between specific indirect effects associated with NA and AOC (Hypotheses 11a-11e)

Table 21. Contrasts between specific indirect effects associated with NA and POS (Hypotheses 12a-12e) 185

Table 22. Contrasts between specific indirect effects associated with NA and LMX (Hypotheses 13a-13e)

Table 23. Contrasts between specific indirect effects associated with NA and IJ (Hypotheses 14a-14e) 186 
Table 24. Contrasts between specific indirect effects associated with AOC and LMX (Hypotheses 15a-15e)

Table 25. Contrasts between specific indirect effects associated with AOC and IJ (Hypotheses 16a-16e)

Table 26. Contrasts between specific indirect effects associated with POS and LMX (Hypotheses 17a-17e)

Table 27. Contrasts between specific indirect effects associated with POS and IJ (Hypotheses 18a-18e)

Table 28a. Moderating effects of PEC on relationship between AS and LMX (Hypothesis 20a)

Table 28b. Moderating effects of PEC on relationship between AS and IJ (Hypothesis 20b) 188

Table 29a. Moderating effects of PEC on relationship between AS and POS (Hypothesis 21a)

Table 29b. Moderating effects of PEC on relationship between AS and AOC (Hypothesis 21b)

Table 30a. Maximum Likelihood Fit Statistics for eight-factor measurement models $(\mathrm{N}=2738)$.

Table 30b. Multiple regression equations estimated with latent variable scores 189

Table 31. Status of study hypotheses. 190

Table 32. Effect sizes and bootstrap standard errors in single and multiple mediator models 


\section{LIST OF FIGURES}

\section{FIGURE}

PAGE

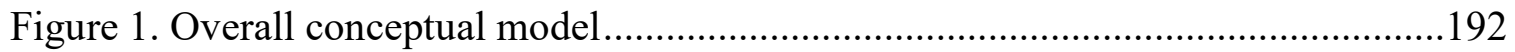

Figure 2. Standardized solution for higher order confirmatory factor analyses of PEC (initial)

Figure 3. Standardized solution for higher order confirmatory factor analyses of PEC (final)......

Figure 4. Conditional effects of AS on LMX at high (1+SD) and low (-1SD) values of PEC

Figure 5. Conditional effects of AS on IJ at high (1+SD) and low (-1SD) values of PEC

Figure 6. Conditional effects of AS on AOC at high (1+SD) and low (-1SD) values of PEC

Figure 7. Abusive supervision item scores by rating source .196 


\section{INTRODUCTION}

\section{Organizational/Workplace Aggression}

Organizational/workplace aggression refers to a broad set of behaviors characterized by the actor's intent to harm (Baron \& Neuman, 1998). Organizational aggression ranges from subtle (negative eye contact, silent treatment) to relatively overt expressions of hostility (homicides, physical assaults; Hershcovis, 2011). Despite having devastating consequences, physical aggression (acts that breach a spatial barrier between perpetrator and victim, i.e., violence; Nixon \& Spector, 2015), represent only a tiny fraction of the aggressive behaviors that occur within US organizations (Schat \& Kelloway, 2003; Schat, Frone, \& Kelloway, 2006). Moreover, acts of physical aggression tend to be perpetrated by persons external to the organization (robbers, relatives of employees) rather than employees (Barling, Dupre, \& Kelloway, 2009).

Until recently (1990s), little research attention was given to the subtle and nonphysical forms of organizational aggression that involve employees and have been shown to be most prevalent in the workplace (Barling et al., 2009; Bowling \& Beehr, 2006). Notable exceptions are sexually and racially motivated aggression (Berdahl, 2007), which in addition to research attention, has also been the subject of intense judicial (Oncale $v$. Sundowner Offshore Service, Inc., 1997) and legislative scrutiny (Civil Rights Act of 1964).

Empirical evidence has consistently shown nonphysical (and nonsexual) forms of aggression (verbal and/or psychological abuse) to be most prevalent in American workplaces (Greenberg \& Barling, 1999; U.S. Postal Service Commission, 2000). For instance, less than $1 \%$ of persons employed in the US become victims of homicide while 
at work and only $6 \%$ of workers in the US report a prior experience of violence (Schat et al., 2006). On the other hand, 1 in 3 American workers report at least one experience of nonphysical organizational aggression (Schat et al.).

In a survey of Human Resource (HR) professionals conducted by the Society for Human Resource Management (SHRM, 2012), most organizations (51\%) had experienced an incident of workplace bullying, defined by the researchers as "persistent, offensive, abusive, intimidating or insulting behavior or unfair actions directed at another individual...". What's more, the most frequently reported type of bullying behavior was verbal abuse (e.g., shouting, yelling, swearing, name- calling), reported by $73 \%$ of HR professionals whose organization had dealt with bullying.

Other studies find that between 71\% (Cortina, Magley, Williams, \& Langhout, 2001 ) and $98 \%$ of workers report experiencing workplace incivility (low intensity [aggression] with ambiguous intent to harm; Anderssen \& Pearson, 1999) in the past year, with half of the workers reporting weekly experiences (Porath \& Pearson, 2013). Overall, the data clearly show the prevalence of less overt forms of aggression in American workplaces far exceeds the prevalence of more extreme acts of workplace aggression (e.g., homicide, physical assault). Moreover, these "mild" acts of aggression have been shown to have a negative impact on the individuals, the organizations they staff (Cortina et al., 2001; Estes \& Wang, 2008; Penney \& Spector, 2005; Welbourne \& Sariol, 2016), and the customers who merely witness these behaviors (Porath, MacInnis, \& Folkes, 2011). The present dissertation is primarily concerned with abusive supervision, a type of workplace aggression characterized by subordinate's perceived aggression from their supervisor. 
Although Tepper (2000) defines abusive supervision as expressions of hostility (unfriendliness, antagonistic), he does not regard abusive supervision as a type of aggression. Tepper clarifies this point by noting that many of the behaviors constituting abusive supervision - because they do not require the actor's intent to harm-are inconsistent with traditional definitions of aggression (Tepper, 2007). The examples he provides however, "a supervisor may mistreat subordinates to elicit high performance or to send the message that mistakes will not be tolerated", appear to fit well within the scope of aggression. Anderson and Bushman (2002) distinguish between proximate (immediate) and ultimate (higher order, distal) goals and suggest it is only necessary that actors have the proximate intent to cause the target harm. Further, they define instrumental aggression as a "premeditated means of obtaining some goal other than harming the victim" (Anderson \& Bushman, 2002, p.121).

Applying Anderson and Bushman's (2002) conceptualization of instrumental aggression to Tepper's (2000) example, supervisors who "mistreat subordinates to elicit high performance" can be said to have a proximate goal of causing harm to the subordinate and an ultimate goal of improving their performance (or promoting rule adherence). This suggests that supervisors may use (instrumental) aggression, in the form of verbal abuse and other non-verbal behaviors consistent with abusive supervision (silent treatment, invasions of privacy, credit taking; Tepper, 2000), to harm the subordinate (proximal goal) and as a means to improve that subordinate's performance (ultimate goal). Consistent with this notion, a recent study by Watkins, Fehr, and He (in press) finds that leaders differ in their instrumentality beliefs regarding abusive supervision and that leaders who believe abusive supervision has a positive impact on employee performance engaged in more 
abusive supervision. Moreover, several researchers (e.g., Mitchell \& Ambrose, 2007; Steinert, 2015; Whitman, Halbesleben, \& Holmes IV, 2014) have conceptualized abusive supervision as interpersonal aggression.

Unlike interpersonal aggression in general-which can occur between coworkers at any level of the organization - the concept of abusive supervision is specific regarding the organizational roles of the parties involved. Indeed, a key aspect of abusive supervision is the inherent power imbalance between the parties that arises from the organizational roles of the victim (subordinate) and perpetrator (supervisor). Research suggest that aggression from supervisors is associated with greater psychological strain than aggression from those perceived to be of equal power (Nixon \& Spector, 2015). Relative to coworkers, supervisors "are uniquely positioned to make available outcomes that many employees find attractive" (Tepper, Carr, Breaux, Geider, Hu, \& Hua, 2009, p. 156). For instance, the supervisor's legitimate position allows him or her to control access to promotions and desirable work assignments (Rupp \& Cropanzano, 2002). Consistent the notion that aggression within supervisor-subordinate relationships produces particularly detrimental outcomes, meta- analysis of organizational aggression from various sources shows aggression from supervisors has stronger (negative) associations with work-related attitudes (job satisfaction, organizational commitment) and work behaviors than aggression from other sources (e.g., outsiders; Hershcovis \& Barling, 2010).

Workplace relationships, however, do not exist in a vacuum. These relationships are shaped by the attitudes and behaviors of each party, and by situational forces internal (e.g., leadership, HR policies, culture, climate) and external (e.g., labor market demands, prestige, natural disasters) to organizations. While organizational culture represents 
implicit values, climate represents shared perceptions of "the way things are done around here" (Vardi, 2001, p. 327).

Organizational climate broadly refers to shared perceptions of formal and informal organizational policies, practices and procedures (Vardi, 2001). Prior research indicates a positive association between organizational aggression and organizational climates marked by hostility and violence (Spector, Coulter Stockwell, \& Matz, 2007). Moreover, aggressive workplace norms and perceived organizational sanctions against aggression have also been positively associated with employee aggression (Inness, LeBlanc, \& Barling, 2008). The impact of ethical organizational climate (EOC) in the context of abusive supervision, however, remains largely unexplored and is the subject of the present investigation.

In the following sections I introduce Ethical Climate Theory (ECT; Victor \& Cullen, 1987) and argue that subordinate's perceived ethical climate (PEC) differentially affects the workplace attitudes under consideration. I then describe various ways in which abusive supervision affects subordinate job performance indirectly and argue for a complimentary approach that reflects the reality that employees hold these various workrelated attitudes simultaneously, and that each has a unique role in predicting shaping subordinate's job performance. Finally, it is argued that PEC moderates the relationship between abusive supervision and these same workplace attitudes to varying degrees.

Employees often develop a shared perception of the criteria organizational members (coworkers, supervisors) use to make ethical decisions (Martin \& Cullen, 2006). ECT (Victor \& Cullen, 1987, 1988) crosses three levels of analysis (individual, local, cosmopolitan) with three stages from Kohlberg's (1984) theory of moral development 
(egoism, benevolence, principle) to arrive a nine possible EOC types. For instance, an EOC characterized by a strong adherence to Rules (i.e., compliance-based) involves the cross between the local level of analysis (where ethical concerns are framed in reference to the team and organization) and the Principle level of moral development (where ethical concerns are based on concern for adherence to personal moral codes).

Subsequent empirical research has identified five of the nine EOCs originally proposed-instrumental, caring, independence, rules and law-and-codes. Moreover, a number of studies show that ethical climate perceptions are associated with work-related attitudes (Cullen et al., 2003; DeConinck, 2011; Hsieh \& Wang, 2016, Mulki, Jaramillo, \& Locander, 2006) and behaviors (Bollman \& Krings, 2016; Leung, 2008; Vardi, 2001).

EOCs differ in how consistent they are with abusive supervision. Simply put, supervisory abuse is more consistent with (and perhaps expected in) organizations with climates characterized by self-serving behaviors and decision-making (e.g., Instrumental EOC) than in organizations with EOCs characterized by mutual goodwill and a consideration of others (e.g., Benevolence EOCs). Therefore, it seems reasonable to expect that when abusive supervision is inconsistent with the organization's ethical climate, attributions of responsibility (causation, control, intentions; Mikula, 1993) for the abuse are more likely to be ascribed to the supervisor, and thereby have a stronger effect on supervisor-referenced attitudes than on organization-referenced attitudes. When the organization's ethical climate is consistent with abusive supervision however, subordinates should be more likely to hold the organization responsible and abusive supervision will have a stronger effect on organization-referenced attituded than on supervisor-referenced attitudes. 
Prior research suggests attributions of responsibility play a role in shaping subordinates' behavioral and attitudinal responses to abusive supervision (Bowling \& Beehr, 2006; Bowling \& Michel, 2011; Wang \& Jiang, 2015). Moreover, Nixon and Spector (2015) found that, among employees who reported frequent experiences of aggression, attributions (of intent to harm) moderated the negative impact of aggression on indicators of psychological strain (i.e., stress-response). Together, these findings suggest that EOC, to the extent they affect how subordinates attribute ambiguous supervisory actions, will moderate the impact of abusive supervision on work-related attitudes.

\section{Subordinate Job Performance}

Although many employee actions (and inactions) can affect organizational functioning, job performance—which is conceptually distinct from "results" (e.g., number of sales, number of publications) —includes three broad dimensions: task performance, organizational citizenship behaviors (OCBs), and counterproductive work behaviors (CWBs). Task (in-role) performance refers to behaviors formally prescribed for an organizational role differs from contextual (extra-role) performance, which subsumes OCBs and CWBs (Miles, Borman, Spector, \& Fox, 2002; Motowidlo \& van Scotter, 1994).

Scholarly interest in abusive supervision has increased dramatically over the last 10 years, with over a dozen investigations reporting on the impact of abusive supervision on subordinate's job performance (Tepper et al., 2017). Prior studies find positive associations between abusive supervision and CWBs (Hussain \& Sia, 2017; Mitchell \& Ambrose, 2007), and negative associations between abusive supervision and OCBs (Decoster, Camps, \& Stouten, 2014) and task performance (Walter, Lam, van der Vegt, Huang, \& Miao, 2015). 
In the following sections I describe six mechanisms, each of which partly explain the effects of abusive supervision on subordinate job performance. These mechanisms do not represent an exhaustive list of all mechanisms advanced in the literature, but instead capture those that are reasonably grounded in theory and have received empirical support. With few exceptions, these mechanisms have been tested in isolation. The contributions of this study are thus twofold: First, the study seeks to determine the extent to which each affect- and attitude-driven mechanism uniquely accounts for the (total) effects of abusive supervision on each job performance dimension, when the other mechanisms are also considered. Second, this study aims to show how well these mediators, as a set, account for the effects of abusive supervision on employee job performance. An additional study contribution will be to establish the role of EOC (as perceived by the subordinate) as a moderator of the relationships between abusive supervision and the subordinate's workrelated attitudes.

In his review of the abusive supervision literature, Tepper (2007) noted that while abusive supervision has been investigated in a variety of settings (e.g., healthcare, paramilitary, military), researchers have yet to investigate how abusive supervision differs across industries. In other words, empirical evidence regarding the contextual factors (e.g., job characteristics, industry effects) associated with abusive supervision is lacking (Martinko et al., 2013). More importantly, there is no evidence to suggest that the relative strength of these mechanisms will be affected by industry factors. That said, the present aim is to determine the relative importance of attitudinal and affective mechanisms in a general population of US workers. 


\section{Mechanisms Linking Abusive Supervision to Subordinate Job Performance}

Abusive supervision has been shown to affect subordinate job performance indirectly through (1) positive affective states, (2) negative affective states, (3) leader-member exchange, (4) interactional justice, (5) perceived organizational support, and (6) affective organizational commitment. These six mechanisms are viewed as complimentary and broadly classified into affective- and judgment/attitude-driven reactions to abusive supervision. Mechanisms 1 and 2 (via PA and NA) represent emotional reactions to abusive supervision while the remaining mechanisms represent the effects of abusive supervision on work-related attitudes related to the supervisor (Mechanisms 3 and 4) and to the organization (Mechanisms 5 and 6). The distinction between affect- and attitude-driven behaviors is a key feature of Affective Events Theory (AET; Weiss \& Cropanzano, 1996).

AET frames workplace events ("things that happen to people in work settings," p. 11) - rather than environmental work features, as the proximal causes of affective reactions. Affective reactions, in turn, shape work attitudes and behaviors. Nonetheless, structural/environmental work features influence affective reactions indirectly (via work events) and directly impact work attitudes. AET proposes that while affect-driven behaviors are a direct result of affective experiences, judgement-driven behaviors are mediated by work attitudes. In other words, while the behavioral effects of abusive supervision, as mediated by affective states, occur relatively immediately, the behavioral effects of AS, as mediated by work-related attitudes, have a longer onset and stem from subsequently thinking about the abuse (Lee \& Allen, 2002; Weiss \& Cropanzano, 1996). It should be noted that job performance includes affect- and judgment-driven behaviors. 
Inherent in the AET framework is the construct of time. That is, AET views work behaviors as shaped by discrete occurrences (i.e., events), rather than by stable environmental factors. While acts of abusive supervision can be observed as discrete events, the persistence of abusive supervision may also come to be seen, at least by some employees, as a stable characteristic of their work environment. AET suggests that while discrete acts of abuse will influence job behavior through affective reactions, persistent/on-going abusive supervision, to the extent it comes to be viewed as a stable job characteristic, will influence job behavior through its effects on work attitudes. As discussed in the following sections, the distinction between affect- and attitude- driven behaviors is a crucial consideration in hypotheses related to the role of ethical climate.

State Affect. The concept of state affect refers to ephemeral emotional experiences and differs from the concept of trait affect (i.e., trait affectivity), which reflects individual differences in disposition to experience affective states that are of a similar valence (e.g., positive, negative) across a variety of contexts. Although plausible, it seems unlikely that abusive supervision will produce changes in individual dispositions, as these tend to remain relatively stable across the lifetime and are likely to already be in place by the time individuals are placed in an organizational role. That said, the present focus is on the momentary experiences of emotions at work (job-related state affect) that are likely to cooccur alongside experiences of supervisory abuse. I operationalize state affect as a selfreported retrospective account of the frequency with which target employees experienced key emotional states at work in the prior 30 days. Importantly, abusive supervision and job performance measures are also operationalized to reflect the same retrospective interval (prior 30 days). 
Acts of abusive supervision represents workplace events which are associated with less frequent positive affective states (e.g., joyful, happy, at ease; PA; Harvey et al., 2007) and more frequent negative affective states (e.g., anxiety, anger, fear; NA; Hoobler \& Hu, 2013; Michel, Newness, \& Duniewicz, 2016). Moreover, PA and NA vary in their relationship to CWBs and OCBs (Lyons \& Scott, 2012; Spector \& Fox, 2002). Specifically, PA and NA are associated with engagement in prosocial behaviors (e.g., OCBs) and behaviors that detract from organizational goals (e.g., CWBs), respectively (Bauer \& Spector, 2015; George, 1991; Khan, Quratulain, \& Crawshaw, 2013).

The decrease in PA and increase in NA that result from abusive supervision are expected to have detrimental effects on subordinates' extra-role performance (decrease in PA decrease OCBs, increase in NA increases CWBs). While PA has been shown to benefit task performance (Tsai, Chen, \& Liu, 2007), the relationship between negative affect and task performance is equivocal. Paradoxically, negative emotional experiences can (1) hinder performance by creating additional cognitive demands (i.e., appraisal, rumination), thereby shifting attentional focus away from the task at hand (Beal, Weiss, Barros, \& MacDermid, 2005) and (2) improve performance by serving as signals (feedback) that things are not going well, thereby mobilizing additional cognitive resources to the task at hand (George \& Zhou, 2002).

Leader-Member Exchange (LMX). Research in organizational leadership has identified key qualities of leader-follower relationships (mutual trust, respect, and perceived obligation) that serve to promote the attainment of organizational goals (Gerstner \& Day, 1997). Specifically, employees that report low LMX view their employment relationship in strictly transactional terms. These employees may refrain from performing 
the extra-role behaviors that help their organizations succeed because they do not trust their supervisor to "return the favor" at a later date. Abusive supervision reflects ongoing downward hostility and is unlikely to be conducive to the development of high-quality relationships between a leader and his or her subordinates. Predictably, the (low-quality) social exchange relationships that follow from persistent supervisory abuse are in turn negatively associated with in-role and extra-role performance.

Interactional Justice (IJ). IJ refers to the fairness of the treatment received from organizational agents (Moorman, 1991). Meta-analytic studies show that while abusive supervision is negatively related to all dimensions of organizational justice (i.e., distributive, procedural, interactional; Colquitt, 2001), it has the strongest (negative) association with IJ (Mackey, Frieder, Brees, \& Martinko, 2017; Zhang \& Liao, 2015). Interactional (in)justice that is experienced as a result of abusive supervision will lead employees to react in ways that diminish their in-role and extra-role performance. As predicted by social exchange theory—an important framework for understanding the effects of justice (Colquitt et al., 2013) — these employees will respond to interactional injustice by reducing their work output (task performance), behaving less generously towards coworkers (OCBs) and engaging in other retaliatory activities (CWBs).

Perceived Organizational Support (POS). Consistent with the idea that supervisors embody the organization, the favorability of the treatment subordinates receive from their supervisors is associated with POS, a general belief regarding how much the organization values and cares about them (Rhoades \& Eisenberger, 2002). In the context of ongoing and persistent abuse from supervisors, some employees may come to view their organization as either incompetent (i.e., unaware and/or unable to provide assistance) or 
complicit (i.e., unwilling to provide assistance) in the abuse. Employees who take this position are unlikely to believe their organization cares about them.

Prior research shows that when employees believe their organization cares about them, they reciprocate by behaving in ways that help organizations meet their goals. Specifically, this includes engaging in interpersonal helping behaviors (OCBs) and refraining from behaviors that detract from organizational functioning (e.g., shirking of duties, CWBs; Eisenberger, Armeli, Rexwinkel, Lynch, \& Rhoades, 2001). Likewise, employees who feel mistreated will also engage in reciprocity. Overall, a negative relation is expected between abusive supervision and POS. In turn, POS is expected to have a negative relationship with CWBs and a positive relationship with OCBs and task performance.

Affective Organizational Commitment (AOC). The favorability of interpersonal treatment received from one's supervisor serves as an indication of one's organizational worth and is associated with AOC (desire to remain with the organization; Meyer \& Allen, 1990). Consistent with prior research (e.g., Tepper et al., 2008), abusive supervision is expected to have a negative relationship with AOC. In turn, AOC is expected to have a positive relationship with task performance and OCBs and a negative relationship with CWBs. AOC has a positive effect on in- role and extra-role performance because individuals emotionally attached to their organization will see the organization's mission and goals as compatible with their own and be willing to behave in ways that align with organizational interests (Cetin, Gurbuz, \& Sert, 2015; Meyer, Paunonen, Gellatly, Goffin, \& Jackson, 1989; Meyer, Stanley, Herscovitch, \& Topolnytsky, 2002; Riketta, 2002). 


\section{Role of EOC}

Past studies also find positive associations between EOC and (1) AOC (Cullen et al., 2003), (2) POS (Hsieh \& Wang, 2016), and (3) trust in the supervisor (i.e., LMX, DeConinck, 2011; Mulki, Jaramillo, \& Locander, 2006). Results consistently show a negative relation between these attitudes and instrumental climates and a positive relation between principled and Benevolent EOCs the same (Martin \& Cullen, 2006). Despite a lack of empirical evidence, IJ is expected to show a similar pattern. That is, Principle and Benevolent EOCs (e.g., Caring, Rules-based) — because they often reference values consistent with the fair treatment of others (i.e., society, coworkers) - should have a positive association with IJ perceptions.

The distinction between affect- (emotional) and cognition-driven (attitudinal) reactions to workplace events (Weiss and Cropanzano, 1996) is key to understanding the moderating role of EOC. Emotional responses to workplace events (e.g., anger) occur quickly and involve less mental deliberation. Responses that are driven by cognition, such as attitude formation/modification, occur more slowly and thus allow individuals to consider additional information. It is precisely when individuals consider additional information that perceived ethical climate (i.e., PEC) will play a role in shaping their response. Stated differently, responses to abusive supervision will be affected by ethical considerations only when individuals have had an opportunity to process the abuse.

Consequently, PEC is expected to interact with abusive supervision to predict attitudinal mediators (i.e., LMX, IJ, AOC, POS) but not affective mediators (PA, NA). Moreover, within attitudinal mediators I further distinguish between attitudes related to the supervisor (LMX, IJ) and attitudes related the organization (AOC, POS). Principled and 
benevolent ethical climates will have an invariably positive association with each of the proposed attitudinal mediators and instrumental climates will have a negative association with the same. Moreover, the relative impact of ethical climate on each work-related attitude will depend on whether the target attitude reflects the organization or the supervisor.

EOC affects how subordinate view the unethical treatment they receive from their supervisors (i.e., abusive supervision). When the organization's ethical climate is perceived to be guided by the care for well-being and the adherence to rules, laws, and a code of ethics (i.e., benevolent and principled climates) abusive supervision (persistent unethical supervisory behaviors) is more likely to blamed on the hostile intentions of the supervisor - thereby enhancing the negative effects of abuse on attitudes with a supervisor focus (e.g., LMX, IJ) to a greater degree than attitudes with an organization focus (e.g., AOC, POS).

In contrast, when the organization's ethical climate is seen as being driven by instrumental concerns, abusive supervision enhances the negative impact of abusive supervision on attitudes towards the organization to a greater degree than attitudes towards the supervisors. Consistent with these expectations, Wang and Jiang (2015) found that when abusive supervision was attributed to the organization, it had no impact on IJ (supervisor-directed attitude). On the other hand, when organizational attributions for abuse were low (i.e., supervisor was blamed), abusive supervision had a negative relationship to IJ.

To my knowledge, only a single study (Biron, 2010) has investigated the effects of abusive supervision and ethical climate simultaneously. In a sample of workers from seven 
Israeli organizations, abusive supervision and organizational ethical climate (operationalized as ethical orientation within the firm) showed a strong negative correlation (-.51; Biron, 2010). Moreover, the generally negative relationship between ethical values and negative work behaviors directed at the organization was attenuated at higher levels of abusive supervision. Biron proposed that the attenuating effect is the result of the supervisor's behavioral inconsistency with the ethical values espoused by the organization. In other words, the lack of supervisor behavioral integrity reduces the salience of the ethical values. As Biron notes, "the degree to which the [supervisor's] words and deeds are consistent may have substantial implications for employee behavior" (p. 890).

In light of prior studies, PEC is expected to moderate the relationship between abusive supervision and work-related attitudes that reference the supervisor (i.e., LMX, IJ), such that the (negative) effects of abusive supervision are stronger when individuals view their coworkers as deferring to principle and benevolence values in their ethical decisionmaking (i.e., benevolence ethical climate) than when they perceive that their coworkers' ethical decisions are guided by self-interest (i.e., instrumental ethical climate). In contrast, abusive supervision is expected to have a stronger (negative) impact on attitudes that reference the organization (i.e., AOC, POS) when an instrumental ethical climate is perceived than when a principle or benevolence ethical climate is perceived.

\section{Chapter Summary}

The research on abusive supervision has matured in the last decade, as marked by increased model complexity (Tepper, et al., 2017) and theoretical developments (Chan \& McAllister, 2014; Klaussner, 2014). With few exceptions (Lian, Ferris, \& Brown, 2012a, 2012b; Priesemuth, Schminke, Ambrose, \& Folger, 2014; Rafferty \& Restubog, 2011; Xu, 
Huang, Lam, \& Miao, 2012; Yu, Lin, Wang, Ma, Wei, et al., 2016), mechanisms linking abusive supervision to subordinate job performance have been studied in isolation and there have been few attempts to evaluate them relative to each other. Despite acknowledging the insights gained from the dozens of studies that have investigated mechanisms linking abusive supervision to distal outcomes (e.g., job performance), Tepper et al. (2007) note,

What is problematic is that the mediation frameworks scholars test typically account for one and rarely more than two mechanisms underlying the effects of supervisory abuse. Consequently, the research being conducted is not able to shed light on the relative explanatory power of the theoretical perspectives that seem relevant to understanding abusive supervision. [emphasis added] (p. 134)

Moreover, although studies that test one or two mechanisms at a time have their use, they allow little to be inferred in terms of the relative explanatory power of the underlying mechanisms and may lead to increased theoretical fragmentation (Tepper et al.).

As the preceding discussion illustrates, there is extant research showing abusive supervision affects subordinate job performance, in part, because (1) abuse decreases the frequency of PA, (2) abuse increases the frequency of NA, (3) abuse is detrimental to subordinate's relationship his or her supervisor (LMX), (4) subordinate's view abuse as a form of unfair interpersonal treatment (IJ), (5) abuse reduces how much subordinates perceive their organization cares about them (POS), and (6) abuse reduces subordinate's affection for, and desire to stay with, their organization (AOC). The primary contribution of this study will be to evaluate the relative strength of these mechanisms.

Finally, PEC is postulated to have a direct effect on work-related attitudes that reference the supervisor (LMX, IJ) and on work-related attitudes that reference the 
organization (AOC, POS). Moreover, PEC is expected to moderate the effects of abusive supervision on work-related attitudes, such that "strong" ethical climates (i.e., characterized by Principle, Benevolence) enhance the negative effects of abusive supervision on supervisor-referenced attitudes (i.e., LMX, IJ) and "weak" ethical climates (e.g., Instrumental EOC) enhance the negative effects of abusive supervision on organization- referenced attitudes (i.e., AOC, POS). 


\section{LITERATURE REVIEW}

This chapter begins with a review of the abusive supervision literature, followed by the development of hypotheses related to the indirect effects of abusive supervision on subordinate task and contextual performance, the latter of which includes organizational citizenship behaviors (OCBs), and counterproductive work behaviors (CWBs) directed at the organization and directed at individuals within the organization (e.g., coworkers). Study hypotheses are derived from evidence that links abusive supervision to each of the proposed mediators and evidence that links each mediator to one or more performance outcomes. Finally, I discuss the concept of ethical organizational climate (EOC; Victor \& Cullen, 1987) and develop hypotheses regarding the impact of subordinates' ethical climate perceptions on the relationship between abusive supervision and key work-related attitudes. Specifically, EOC is expected to influence the strength of the relationships between abusive supervision and work-related attitudes.

\section{Abusive Supervision: Subordinate-targeted Aggression}

Organizational aggression differs from the related concept of occupational violence by being "organization-motivated" (precipitated by factors within the control of the organization; O'Leary-Kelly, Griffin, \& Glew, 1996) and can have a variety of sourcesincluding those that are internal (e.g., coworkers) and external (e.g., general public, customers; Barling et al., 2009) to the organization. Relative to other sources, it can be argued that aggression from the immediate supervisor is more concerning to employees because it threatens harm to employee's organizational status. Unlike coworkers and subordinates, supervisors are uniquely positioned to harm through their control over work

schedules, assignments, and access to organizational resources and developmental 
opportunities (mentoring, training). Consistent with this notion, meta-analytic evidence shows aggression from supervisor (i.e., targeting subordinates) to be more strongly associated with the subordinate outcomes of interest (job performance) than aggression from coworkers or outsiders (Hershcovis \& Barling, 2010).

\section{Defining Abusive Supervision}

The concept of abusive supervision (Tepper, 2000) reflects organizational aggression "from above" (Hershcovis \& Barling, 2010; Mitchell \& Ambrose, 2007). Specifically, abusive supervision refers to "the sustained display of hostile verbal and nonverbal behaviors, excluding physical contact" (Tepper, 2000, p. 178). Although not explicit in the definition, abusive supervision notably reflects the subordinate's subjective assessment of the supervisor's behavior rather than an objective indicator of the supervisor's behavior.

As Tepper (2000) notes, “The same individual could view a supervisor's behavior as abusive in one context and as non-abusive in another context, and two subordinates could differ in their evaluations of the same supervisor's behavior" (p.178). Indeed, in some organizational contexts (e.g., police agencies), many of the behaviors included in Tepper's (2000) scale could be interpreted as "tough love" rather than "abusive" (Simon Hurst, Kelley, \& Judge, 2015). Throughout this manuscript, “abusive supervision" refers to perceptions of (having been the target of) and/or perceived abusive supervision. Following the tradition of Tepper and colleagues (2017), the words "perceived" and "perceptions of" are omitted to produce a less cumbersome prose. 


\section{Conceptual and Methodological Issues}

Extant research on interpersonal aggression has identified many constructs that are conceptually distinct yet tend to be operationalized in similar ways (Hershcovis, 2010). A partial list includes interpersonal deviance (Bennett \& Robinson, 2000) social undermining (Duffy, Ganster \& Pagon, 2002), incivility (Andersson \& Pearson, 1999), rudeness (Porath \& Erez, 2007), abusive behavior (Keashly, Trott, \& MacLean, 1994), emotional abuse (Keashly, 1997), workplace harassment (Bowling \& Beehr, 2006), victimization (Aquino \& Bradfield, 2000) and workplace mistreatment (Cortina \& Magley, 2003). In addressing theoretical fragmentation and cross-proliferation concerns, several researchers (e.g., Hershcovis, 2010; Aquino \& Thau, 2009) suggest the need to measure the precise dimensions that differentiate the target concept from similar and broader concepts (i.e., organizational aggression, workplace mistreatment).

Baillen, Escartin, Gross, and Zapf (2017) differentiated between workplace bullying (Leymann, 1996) and interpersonal conflict (Spector \& Jex, 1998) on the basis of frequency, duration, and power imbalance. Indeed, many related interpersonal aggression concepts can be distinguished (at least theoretically) based on their inclusion of a physical or sexual component, visibility (extent to which they are witnessed by non-targets), intensity, frequency, duration, and relationship power between victim and perpetrator (cf. Hershcovis, 2010). In their investigation of workplace aggression, Nixon and Spector (2015) found that greater psychological strain (psychosomatic complaints, depression) was reported by persons who experienced aggression more frequently, more intensely, perceive the aggression to be visible to others, and when the aggressor has greater relationship power. Using Nixon and Spector's framework, abusive supervision could be described as 
a type of nonsexual, non-physical aggression which occurs with high frequency and duration, and is characterized by a power imbalance between parties.

Several methodological issues arise when the relationship between abusive supervision and subordinate job performance are investigated. One methodological issue concerns the validity of supervisor ratings of performance in the context of abusive supervision is questionable. Tepper et al. (2017) argued that supervisors can express hostility by rating the subordinate lower than he or she deserves, such that abusive supervision and supervisor performance ratings may represent two indications of the same construct. Although dozens of investigations have found associations between abusive supervision and job performance, only two studies (described below) have followed Tepper et al.'s recommendations to employ more objective measures of performance (e.g., Yu, Lin, Wang, Ma, \& Wei, et al. 2016; Walter, Lam, van der Vegt, Huang, \& Miao., 2015).

An additional methodological concern arises from the use of cross-sectional designs, which despite being unable to support definitive causal claims, comprise a large portion of the abusive supervision literature. That said, while subordinate performance is presently framed as an outcome of abusive supervision, the temporal precedence of abusive supervision and subordinate job performance remains controversial. Moreover, a diverse set of theoretical perspectives (Victimization Theory; Aquino \& Thau, 2009; Employee Maintenance Theory; Tepper \& Simon, 2015) and empirical investigations (e.g., Eissa, Lester, \& Gupta, 2019; Khan, Moss, Quratulain, \& Hameed 2016; Lian, Ferris, Morrison, \& Brown, 2014; Tepper, Moss, \& Duffy, 2011) have subordinate's characteristics and behaviors as causes of abusive supervision. 
Yet a third possibility is that abusive supervision and subordinate behaviors (such as job performance) are reciprocally related, such that the supervisor's abusive reactions to the subordinate's (poor) performance precipitates further declines in performance. Indeed, this reciprocal relationship has been described as "cycles of abuse" (Simon et al, 2015) and "escalating spirals" (Klaussner, 2014) and is made explicit in two recent theories of abusive supervision (e.g., Chan \& McAllister, 2014; Klaussner, 2014). However, there exists no theoretical rationale to suggest what factors influence the tempo of these cycles. For instance, Lian et al. (2014) found that CWBs directed at the organization (CWBO) and abusive supervision were reciprocally related at six months but not at twenty months. More research is clearly needed to understand the influence of time in the dynamic interaction between subordinate job performance and abusive supervision.

\section{Individual-level Outcomes}

The concept of abusive supervision has generated hundreds of investigations in the nearly two decades since Tepper's (2000) introduction. Arguably, the most active area of the literature concerns the (mostly negative; cf. Lee, Yun, \& Srivastava, 2013) subordinatelevel consequences of abusive supervision (Tepper et al., 2017). Abusive supervision has been linked to negative personal outcomes including problems with alcohol (Bamberger, 2006), conflict with family members (Carlson, Ferguson, Hunter \& Whitten, 2012; Carlson, Ferguson, Perrewé, \& Whitten, 2011), spousal undermining (Restubog, Scott \& Zagenczyk, 2011), subjective well-being (Bowling \& Michel, 2011), psychological distress (Li, Wang, Yang, \& Liu, 2016), emotional exhaustion (Aryee et al., 2008; Wu \& $\mathrm{Hu}, 2009$; Yagil, 2006), and a variety of subclinical psychological complaints such as anxiety and depression (Tepper, Moss, Lockhart, \& Carr, 2007). This non-exhaustive list 
suggests that the negative consequences of abusive supervision extend beyond the workplace - affecting personal health and relationships with persons outside of work.

\section{Abusive Supervision and Subordinate Job Performance}

In addition to personal and family outcomes, abusive supervision also shapes attitudes and behaviors at work. Job performance refers to employee behaviors "... that are linked with and contribute to organizational goals" (Viswesvaran \& Ones, 2000, p.216). Job performance includes three broad dimensions: task performance, organizational citizenship behavior (OCBs), and counterproductive work behaviors (CWBs). Task performance supports the organization's technical core and contributes to organizational effectiveness. Task performance is further distinguished from contextual performance (extra-role performance), which includes OCBs and CWBs (Miles, Borman, Spector, \& Fox, 2002; Motowidlo \& van Scotter, 1994).

While OCBs make positive contributions to organizational effectiveness, CWBs contribute negatively to organizational effectiveness. It is crucial to note that OCBs and CWBs represent theoretically and empirically distinct performance dimensions rather than opposite poles of a single dimension (Dalal, 2005; Dalal, Lam, Weiss, Welch \& Hulin, 2009; Spector, Bauer, \& Fox, 2010). For instance, Dalal (2005) reported a population estimate regarding the relationship between OCB and CWB of $p=-.32(\mathrm{k}=49, \mathrm{n}=16)$, a magnitude that many organizational researchers would consider as evidence of their independence. Moreover, an experimental study conducted by Spector et al. (2010) suggests that many of the strong negative correlations reported in the literature may be the product of measurement artifacts (e.g., use of overlapping item sets, agreement response format, supervisor- [vs. employee-] rated). 
Interestingly, both the CWB and OCB literatures distinguish between extra-role behaviors that target the organization $(\mathrm{OCBO}, \mathrm{CWBO})$ and extra-role behaviors that target individuals within the organization (OCBI, CWBI; Berry, Ones, \& Sackett, 2007; Coleman \& Borman, 2000; Podsakoff, Whiting, Podsakoff, \& Blume, 2009; Robinson \& Bennett, 1995). Consistent with these theoretical developments and prior empirical findings, the present study distinguishes between, and makes separate predictions for, the interpersonaland organization-directed dimensions of contextual performance.

\section{Task Performance}

Relatively few studies have investigated the effects of abusive supervision on subordinate task performance. Indeed, Tepper's (2007) review of abusive supervision literature revealed a single study of US automotive employees (Harris, Kacmar, \& Zivnuska, 2007) in which it was reported that the negative effects of abusive supervision on subordinate task performance (supervisor-rated) manifested only in persons that attached high meaning to their work.

Walter et al.'s (2015) study found a negative relationship between abusive supervision and objective job performance (Chinese garment workers production output). Interestingly, the effects of perceived subordinate performance (supervisor-rated) predicted abusive supervision only under conditions of low outcome dependence (supervisor-rated). In addition, the negative effect of abusive supervision on subsequent objective performance (1 month after) was not significant after controlling for prior objective performance.

Further, Nandkeolyar et al. (2014) observed an interaction between abusive supervision and employee conscientiousness such that the negative relationship between 
abusive supervision and job performance existed only for employees with low (i.e., lower than .76 SD below mean) conscientiousness. Two studies (e.g., Jian, Kwan, Qiu, Liu et al., 2012; Xu, Huang, Lam, \& Miao, 2012) have explored the relationship between abusive supervision and task performance using supervisor-subordinate dyads.

Jian et al.'s (2012) study of 354 dyads working in various Chinese hotels showed a negative relationship between abusive supervision and service performance. Similarly, Xu et al.'s (2012) study of 366 dyads working for a Chinese garment company also revealed a negative relationship between abusive supervision and subordinate task performance. Finally, Mackey, Frieder, Brees, and Martinko's (2017) meta analytic study estimated a population correlation of between abusive supervision and task performance of $p=-.19$.

\section{Organizational Citizenship Behaviors}

Several investigations have reported negative associations between abusive supervision and general measures of OCBs (Chu, 2014), citizenship behaviors directed towards organizational members (OCBI; Aryee, Chen, Sun, \& Debrah, 2007; Decoster, Camps, \& Stouten, 2014), the organization proper (OCBO; Aryee et al., 2007; Decoster, et al, 2014) and customers of the organization (Lyu, Zhu, Zhong, \& Hu, 2016). Interestingly, Zellars, Tepper, and Duffy (2002) found that the relationship between abusive supervision and OCBs was stronger for those who defined OCB as extra-role behavior.

Abusive supervision has also been linked to narrower facets of OCBs such as helping coworkers (Peng, Schaubroeck, \& Li, 2014), prosocial voice and silence behaviors (Rafferty \& Restubog, 2011), civic virtue (Shoss et al., 2013), and interpersonal facilitation and job dedication (Aryee, Sun, Chen, \& Debrah, 2008). Furthermore, meta-analytic 
studies have observed a negative relationship between abusive supervision and OCBs (Mackey et al., 2017) and between abusive supervision and OCBI and OCBO (Zhang \& Liao, 2015).

\section{Counterproductive Work Behaviors}

Relative to the other job performance dimensions currently investigated (task, OCBs), a much larger portion of the research on abusive supervision has focused on its links to counterproductive behaviors (Martinko et al., 2013). For instance, in Martinko et al.'s (2013) review of the abusive supervision literature, fifty percent of the thirty-four studies that examined outcomes of abusive supervision investigated aggressive (i.e., counterproductive) responses (attitudes and behaviors). Overall, evidence from over a dozen empirical investigations suggest a positive link between abusive supervision and CWBs (Tepper et al., 2017).

The overarching conclusions gleaned from these studies is that abusive supervision has a positive association with CWBs that target the organization and with CWBs that target individuals within the organization (CWBI/CWBO; Tepper et al., 2015; Martinko et al., 2013). Meta-analytic estimates (Mackey et al., 2017; Zhang \& Liao, 2015) of the association between abusive supervision and CWBI suggest a population correlation ranging from .35 from .37 and a population correlation between abusive supervision and CWBO ranging from .38 to .41 .

\section{Problem Statement and Study Contributions}

Scholarly interest in abusive supervision has increased dramatically over the last 10 years (see Tepper, et al., 2017), with over a dozen investigations reporting on the impact of abusive supervision on subordinate's extra-role performance. The following sections 
describe three types of variables - affective states, attitudes towards supervisor, attitudes towards organization - that account for the effects of abusive supervision on subordinate job performance. This study makes the following contributions: (1) provides a mediation framework to test the relative influence of these mediators and, (2) establishes the role of EOC (Victor \& Cullen, 1987) as a boundary condition vis-a-vis the strength of the relationship between abusive supervision and attitudes directed at the supervisor and organization.

In their review of studies that have examined the effects of abusive supervision on distal outcomes (e.g., behaviors), Tepper et al. (2017) suggest that progress in this area has been impeded piecemeal examination of explanatory mechanisms. Specifically, the authors note that,

the mediation framework scholars test typically account for one and rarely more than two mechanism underlying the effects of supervisory abuse. Consequently, the research being conducted is not able to shed light on the relative explanatory power of the theoretical perspectives that seem relevant to understanding abusive supervision (p.134)

The connections between abusive supervision and each mediator discussed below are theoretically grounded and have varying degrees of empirical support. However, with few exceptions (e.g.,, Peng, Schaubroeck, \& Li, 2014; Rafferty \& Restubog, 2011; Xu, Huang, Lam, \& Miao, 2012, Yu, Lin, Wang, Ma, Wei, et al., 2016), theoretically similar mechanisms have been evaluated separately, with few attempts to compare their relative strength (i.e., explanatory power) within a single sample. Determining the relative contribution of mechanisms that rely on similar processes by testing them simultaneously will provide evidence of their unique contribution and add clarity to the abusive supervision literature. 
The present study addresses these concerns by testing the relative explanatory power of six mechanisms that represent diverse theoretical perspectives. The diversity of mechanisms involved in shaping behavioral reactions to abusive supervision suggests that a variety of interventions are possible to reduce the occurrence of abusive supervision and/or mitigate the negative effects of abuse. Therefore, the knowledge gained from this study — in terms of the relative importance of affect and attitudinal mechanisms - is precisely the type of information organizational decision makers need to design and implement the most effective interventions. The full conceptual model is presented in Figure 1.

The second contribution is extending ECT (Victor \& Cullen, 1987) to the study of abusive supervision by showing that EOC moderates the relative impact of abusive supervision on supervisor and organizational attitudes. Existing empirical evidence links abusive supervision to EOC and to attitudinal mediators under present investigation. This study is the first to investigate how attitudinal behavioral reactions to abusive supervision are shaped by subordinate's perceptions of various EOC types. As discussed below, distinguishing mechanisms which are the product of affective reactions from those driven primarily by cognition (i.e., attitudes) is crucial to understanding the moderating effects of EOC.

The present study examines the extent to which specific behavioral consequences of abusive supervision, namely job performance, are the result of (1) affective experiences (PA, NA) at work, (2) attitudes that develop towards the organization (AOC, POS) and (3) attitudes that develop towards the supervisor (LMX, IJ). Moreover, I measure job performance via coworker (peer) ratings, which have been shown to converge with 
supervisor ratings at the construct level (Viswesvaran, Schmidt, \& Ones, 2002) and raise fewer objectivity concerns than supervisory ratings of subordinate performance when it is assessed in the context of an abusive supervisor (see Tepper et al., 2017).

Meta-analytic estimates of the relationship between abusive supervision and each attitudinal mediator and job performance outcome under investigation are presented in Table 1. As shown on Table 1, all work-related attitudes under investigation have a negative relationship with abusive supervision, and abusive supervision has a stronger relationship with attitudes that reference the supervisor (LMX, IJ) than with attitudes that reference the organization (AOC, POS). This suggests that all things other being equal, abusive supervision should have a stronger influence on attitudes towards the supervisor than on attitudes towards the organization. Moreover, Table 2 lists meta-analytic estimates of the relationships between each mediator and employee job performance. Overall, the pattern of correlations shown on Tables 1 and 2 suggest the effects of abusive supervision are stronger for contextual performance than for task performance.

In the sections that follow, I review the empirical and theoretical support for each mechanism linking abusive supervision to the subordinate job performance outcomes. This is followed by a review of ECT and prior investigations that link EOC to abusive supervision and to the attitudinal mediators under present investigation. As previously noted, abusive supervision is negatively related to subordinate in-role and extra-role performance, and these negative relationships are mediated by (1) the experience of affective states (PA, NA), (2) attitudes towards the supervisor (LMX, IJ), and (3) attitudes towards the organization (POS, AOC). Moreover, EOC is related to abusive supervision 
and work-related attitudes (POS, AOC, LMX, IJ) and (2) interacts with abusive supervision to shape the same work-related attitudes.

Based on the arguments articulated in the sections that follow, abusive supervision and LMX and IJ should have a stronger negative relationship when subordinates perceive their firm's EOC is characterized by a concern for others (e.g., Principle, Benevolence) than when EOCs are characterized by a focus on individual's self-interest (e.g., philosophy of Egoism). On the hand, the negative relationships between abusive supervision and POS and AOC should be stronger when EOCs focus on individual's self-interest than when they focus on the interests of others.

\section{State Affect versus Attitudes}

The distinction between state affect and attitudes-summary evaluations of a psychological object along attributes such as good-bad, harmful-beneficial (Ajzen, 2001) - is not without controversy. Addressing prior research in job satisfaction, Weiss (2002) argued that while attitudes have both an affective and a cognitive component (i.e., beliefs), they are distinct from pure affective states. He then summarized the empirical support for the distinction between affect and attitudes in measures of job performance into three categories: (1) factor-analytic studies showing attitudes and affect measures load on separate factors, (2) studies showing that affect and beliefs independently predict overall job performance (i.e., global attitude), and (3) evidence that affective states, but not beliefs, mediate the relationship between trait affectivity and job satisfaction. Indeed, the leading view of attitudes is that they have distinct cognitive and affective components (Ajzen, 2001; Eagly \& Chaike, 1993; Petty, Wegener, \& Fabrigar, 1997). 
More importantly, while the behavioral consequences of affective states are immediate and unmitigated by cognitions, the behavioral consequences of attitudes reflect planning and deliberation (Ajzen, 1991). Weiss (2002) further states that "many, if not most, relationships between affect and work behaviors are not mediated by any overall evaluation of the job" (p. 185). Although his comments referred specifically to the affective component of job satisfaction — researchers have used similar logic to distinguish between the affective and cognitive bases of reactions to injustice (Weiss, Suckow, \& Cropanzano, 1999), and between the cognitive and affective antecedents of organizational citizenship behaviors (Lee \& Allen, 2002; Lyons \& Scott, 2012) and workplace deviance (Judge, Scott, \& Ilies, 2006; Lee \& Allen, 2002). Results from these studies do not provide a clear answer to which types of mechanisms play a stronger role in shaping behaviors and instead suggests a complex interplay between affect and cognition in shaping specific behavioral outcomes. For instance, Lee and Allen (2002) found that the relative contribution of affect and job-related cognitions (justice, pay) depended on the contextual performance outcome investigated (OCBs, CWBs) and how affect was conceptualized.

Affective experiences are distinguished based on valence (positive vs. negative), a distinction which is prevalent in the organizational behavior literature (Tellegen, Watson, \& Clark, 1999), and is practical considering the outcomes of interest. An important feature of affective experiences is that they take control precedence, which refers to "the phenomenon whereby affective states consume cognitive resources, diverting attention, reasoning, and memory to the affective state being experienced" (Lyons \& Scott, 2012, p.69). Lyons and Scott further note that affective experiences disrupt the rational tracking of inputs and outcomes that characterize exchange relationships, leading to behaviors that 
may or may not align with reciprocity norms. These findings suggest that (1) affective experiences resulting from abusive supervision are the product of action-tendencies and will have immediate behavioral consequences, and (2) neither the affective experience itself nor its behavioral consequences should be moderated by subordinate's EOC perceptions. In other words, while EOC perceptions are expected to moderate the effect of abusive supervision on work-related attitudes, they should not moderate the effects of abusive supervision on the experience of affective states (PA, NA).

\section{Supervisor- versus Organization-referenced Attitudes}

Attitudes can be formed regarding any attitude object (Ajzen, 2001). Attitudes are not mere descriptions of the work environment, nor are they the same as perceptions (Judge \& Kammeyer-Mueller, 2012). As noted by Judge et al. (2012), "these constructs [perceptions, descriptions] are not attitudes if they do not include an explicit appraisal or evaluation of the object in question" (p. 346). In the workplace, individuals can develop attitudes towards virtually any facet of their job-ranging from objects in their physical environment (e.g., office furniture, factory equipment) to the more abstract features of organizational life (e.g., promotional opportunities, task meaningfulness). AOC and POS reflect employee attitudes towards (i.e., summary evaluations of) the organization. Specifically, POS captures evaluations regarding how much employee believe the organization values them and AOC reflects how much employees value their organization.

Although AOC represents the most affective component of organizational commitment, its attitudinal properties are readily apparent in widely used measures of AOC. For instance, Allen and Meyer's (1990) measure of AOC asks respondents to indicate the extent to which they endorse items such as, "This organization has a great deal 
of personal meaning for me." and "I enjoy discussing my organization with people." These items have a clear cognitive component and suggest AOC can conceptualized as an attitude that captures employees' evaluations of their organization.

In contrast to AOC and POS however, the attitude object referenced by LMX and IJ is the supervisor. It is important to note that attitudes towards the organization and attitudes towards the supervisor may share a variety of antecedents and thus likely to covary. Moreover, prior research (e.g., Rupp \& Cropanzano, 2002) and intuition suggests that the actions of the supervisor influence not only evaluations of the supervisor, but also evaluations of the organization. Eisenberger et al. 's (2010) concept of supervisor organizational embodiment (perceived similarity between characteristics of supervisor and organization; SOE) is particularly useful to understanding why supervisory behaviors affect employee attitudes towards the organization.

Supervisory functions often include carrying out tasks on behalf of the organization (e.g., staffing, discipline), which lead employees to view supervisors as organizational agents. When subordinates view their supervisors as organizational agents, the supervisor's actions - even those harmful to employees - are seen as being aligned with the organization's objectives. Indeed, mistreatment by the supervisor is interpreted as mistreatment by the supervisor on behalf of the organization. Shoss et al. (2013) found that POS mediated the relationship between abusive supervision and subordinate extra-role performance (OCBs, CWBs). More importantly, the effect of abusive supervision on extrarole behaviors was conditional on SOE, such that subordinates were more likely to hold the organization accountable for the mistreatment (by engaging in negative extra-role 
behaviors) when supervisors embodied the organization (i.e., high SOE) than when they did not (i.e., low SOE).

\section{Affective Mechanisms Linking AS to Job Performance}

There is considerable debate regarding the structure and dimensionality of affect (Carver \& Harmon-Jones, 2009; Shockley, Ipsas, Rossi, \& Levine, 2012). Although much of the discussion is beyond the current scope, the reader should note that affect is a broad umbrella term used to describe a range of emotions, moods, and dispositions. Affect differs from affectivity however, with the latter term referring to individual dispositions to experience specific emotions consistently across time and situations (Barsky \& Kaplan, 2007; Kaplan, Bradley, Luchman, \& Haynes, 2009).

The hierarchical model of affect (Tellegen, Watson, \& Clark, 1999) positions dimensional state affect (PA and NA) below a general "hedonic-evaluative" component. Prior research indicates that abusive supervision is (1) positively associated with the experience of negative affective states such as anger, fear, anxiety, frustration, and embarrassment (Atwater, Kim, Witt, Latheef, Callison, Elkins, \& Zheng, 2016; Hoobler \& Hu, 2013; Michel et al., 2016) and (2) negatively associated with the experience of positive affective states such as interested, enthusiastic, and attentive (Harvey, Stoner, Hochwarter, \& Kacmar, 2007). Interestingly, a recent study by Peng, Schaubroeck, Chong, and Li (in press) reports that while abusive supervision is associated with discrete emotions of anger, shame, and fear, only the behavioral action tendencies associated with anger were in turn related to higher employee CWBI. Although these studies demonstrate the merits of conceptualizing discrete emotions, relatively few discrete emotions have received research attention in the context of abusive supervision - with efforts heavily skewed in favor of 
emotions with a negative valence (e.g., anger, fear; Oh \& Fahr, 2017). Hypotheses are therefore formulated at the broader level of dimensional state affect (i.e., PA and NA).

\section{Abusive Supervision and State Affect}

As Tepper and Almeda (2012) note, instances of abusive supervision can be viewed as affective events that "evoke momentary fluctuations in state negative affect and distal reactions in the form of redress" (p. 87). The present expectations - that PA and NA mediate the effects of abusive supervision on employee performance - are consistent with AET (Weiss and Cropanzano, 1996) and with Spector and Fox's (2002) Theory of Voluntary Work Behavior-which independently propose that affective states mediate the effect of work events/environmental conditions on employee performance. Note that in contrast to AET, the voluntary work behavior model explicitly adopts the two-factor (independence) view of affect.

The implications of the two-factor approach for the present investigation concern expectations regarding the relationship of PA and NA with CWBs and OCBs. Specifically, Spector and Fox's (2002) model predicts that while NA primarily influences CWBs, PA primarily influences OCBs. Miles et al. (2002) found general support for the dual mechanisms proposed by Spector and Fox's model, as have later investigations (e.g., Dalal et al., 2009) reporting differences in the antecedents and consequences of PA and NA (and the discrete emotions associated with each). Interestingly, Yagil, Ben-Zur, and Tamir (2011) found abusive supervision to be related to high NA but unrelated to low PA.

PA and Job Performance. PA is thought to influence task performance through motivational, interpersonal (Tsai, Chen, \& Liu, 2007), and self-regulatory processes (Beal, Weiss, Barros, \& MacDermid, 2005). The motivational benefits of PA on task performance 
are primarily the result of employees' expectancy, instrumentality, and valence considerations (Erez \& Isen, 2002). Erez and Isen's experimental study showed a connection between PA, three components of motivation — valence, instrumentality, and expectancy - and task performance. Specifically, results suggest that employees who experience PA will feel greater attraction towards organizationally-relevant rewards (valence), perceive a clearer connection between their behavioral efforts and success on the task (expectancy) and between their success on a task organizationally-relevant rewards (instrumentality).

PA also exerts a positive influence on in-role and extra-role performance through interpersonal processes. Employees who experience positive moods are more likely to elicit similar emotions in others (e.g., coworkers) and as a result they are more likely receive help from those persons when they need it (Lyons \& Scott, 2012). Lyon and Scott, guided by social exchange theory, argue that employees who feel they have received help from others (or have been harmed by others) are likely to reciprocate helping (and harming) behaviors. Finally, PA may negatively influence task performance because it pulls cognitive resources away from the task at hand (Beal et al., 2005).

Empirical investigations have shown a positive link between PA and task performance and between PA and OCBs (George, 1991; Miner \& Glomb, 2010; Shockley et al., 2012). Interestingly, several studies (e.g., Dalal, et al., 2009; Fisher, 2002; Lyons \& Scott, 2012; Shockley et al., 2012) find that while PA predicts OCBs well, it has a weak relationship to CWBs. The existing research supports the following expectation:

H1: PA mediates the relationship between AS and (a) Task Performance, (b) OCBO, (c) OCBI, (d) CWBO, (e) CWBI. 
NA and Job Performance. NA affects task performance through motivational, interpersonal, and self-regulatory processes. McColl-Kennedy and Anderson (2002) provide evidence that feelings of frustration due to the supervisor's behavior reduce task performance because they lead to feelings of hopelessness and the subordinate essentially gives up trying to perform well (i.e., motivational process). Moreover, action-tendencies that accompany NA are generally antisocial—suggesting that employees who experience NA are likely to push others away and thus less likely to receive help from coworkers when needed (Lyons \& Scott, 2012).

Finally, Self-Regulatory Theory (Muraven \& Baumeister, 2000) suggests that the experience of emotional states inhibits task performance because processing emotions draws upon cognitive resources (e.g., attention, memory) that could otherwise be allocated towards the task at hand (Beal, Weiss, Barros, \& MacDermid, 2005; Collins \& Jackson, 2015). Shockley et al., (2012) meta-analysis also found a negative relationship between NA and the negative discrete emotions that characterize NA and task performance.

Shockley et al.'s (2012) meta-analysis also link NA to employee contextual performance. Specifically, NA, and the discrete emotions that characterize NA, have positive relationships with CWBs and negative relationships with OCBs (Shockley et al.). Additional research has shown that the effects of NA on contextual performance are the product of specific action-tendencies associated with the discrete emotions that characterize NA (e.g., anger is associated with attack-tendency; Colquitt et al., 2013), which facilitates engagement in CWBs but hinders engagement in OCBs because it reduces interpersonal attraction towards (i.e., liking for) coworkers and the organization. Consistent with Spector and Fox's (2002) model of voluntary work behavior, simultaneous 
investigations of PA and NA and contextual performance have shown that while NA predicts CWBs well, it predicts OCBs weakly (Dalal, 2005; Shockley et al., 2012) or not at all (Dalal et al., 2009; Fisher, 2002).

NA has been shown to mediate the relationship between abusive supervision and CWBs (Avey, Wu, \& Holley, 2015; Fox, Spector, \& Miles, 2002; Michel, Newness, \& Duniewicz, 2017). Similarly, state hostility (discrete emotion characteristic of NA) was found to mediate the effect of interpersonal justice (i.e., unfair treatment) on CWBs (Judge, Scott, \& Ilies, 2006). The indirect effects of abusive supervision on OCBs and task performance (through NA) have yet to be shown empirically. However, these effects are theoretically congruent with prior studies demonstrating that the experience of negative affective states (frustration, contempt) mediates leaders' behaviors (e.g., leadership style) on subordinate task performance (McColl-Kennedy \& Anderson, 2002) and OCBs (Sund $\&$ Lines, 2017). The state of the literature suggests the following:

H2: Negative Affect mediates the relationship between AS and (a) Task Performance, (b) OCBO, (c) OCBI, (d) CWBO, (e) CWBI.

\section{Supervisor-referenced Attitudes Linking AS to Job Performance}

\section{Leader-Member Exchange}

At the core of Leader-Member Exchange Theory (LMX; Graen \& Schiemann, 1978 ) is the notion that the quality of the leader-follower relationships varies across followers. While high quality exchanges are marked by high levels of trust, respect, and obligation, low quality exchanges are characterized by low levels of the same dimensions (Graen \& Uhl-Bien, 1995). Abusive supervision refers to specific supervisory behaviors (e.g., verbal, nonverbal hostility). In contrast, LMX characterizes the overall quality of the 
supervisor-subordinate relationship (Xu, Loi, \& Lam, 2015). That said, abusive supervision can occur within the context of an otherwise high-quality relationship (Lian, Ferris, \& Brown, 2012b; cf. Martinko, Harvey, Sikora, \& Douglas, 2011).

Prior studies show that LMX has a positive relationship with task performance (Gerstner \& Day, 1997) and mediates the relationship between abusive supervision and task performance (Xu et al., 2012) —although a one recent study failed to find an indirect effect of LMX once affective commitment and emotional exhaustion were accounted for as competing mediators (Yu et al., 2016). LMX has a direct positive effect on OCBs (Ilies, Nahrgang, \& Morgeson, 2007), and mediates the effects of abusive supervision on OCBs and task performance (Xu et al., 2012). In a replication of Xu et al.'s (2012) study however, Decoster, Camps and Stouten (2014) confirmed that LMX mediated the effects of abusive supervision OCBI and OCBO but failed to find the mediating role of LMX for the abusive supervision-task performance relationship.

Empirical evidence to support the expected indirect effects of abusive supervision on task performance (via LMX) is equivocal. However, such a prediction is congruent with social exchange and justice- based explanations, which tend to suggest that a lack of trust and mutual respect for the supervisor will lead workers to exert less effort on their jobspresumably because they lack confidence that the organization or the supervisor will repay them later. Finally, the mediating role of LMX in the abusive supervision-CWB relationship remains untested, but is suggested by meta-analytic studies that show negative associations between (1) abusive supervision and LMX (Mackey et al., 2017), and (2) LMX and CWBs (Martin, Guillaume, Thomas, Lee, \& Epitropaki, 2016). 
H3: Leader-member exchange mediates the relationship between AS and

(a) Task Performance, (b) OCBO, (c) OCBI, (d) CWBO, (e) CWBI.

\section{Interactional Justice}

Organizational justice theorists (Colquitt, 2001; Moorman, 1991) have suggested that individuals form distinct justice evaluations regarding (1) the favorability of their resource exchange with the organization relative to others (distributive justice), fairness in the procedures used by the organization to make allocation decisions (procedural), and fairness in the way the decisions are implemented and are treated by the organizational agents who implement the procedures (interactional justice). Malatesta and Byrne (1997) suggest that immediate supervisors - because they administer policies and procedure (i.e., ensuring rule compliance) — represent a primary source of interactional justice. Consistent with this notion, a study by Aryee et al. (2007) found that interactional justice (but not procedural justice) mediated the effect of abusive supervision on OCBs.

Abusive supervision violates the rules of conduct and workplace norms regarding equitable treatment (Tepper, 2000). Empirical support for this claim comes from metaanalyses that find strong negative associations between interactional justice and abusive supervision (Mackey et al., 2017; Zhang \& Liao, 2015; see Table 1). Moreover, metaanalytic studies have also shown that interactional justice has (1) a negative association with CWBs, (2) a positive association with task performance, and (3) a positive association with OCBs (Berry, Ones, \& Sackett, 2007; Cohen-Charash \& Spector, 2001; Colquitt, Scott, Rodell, Long, Zapata, et. al., 2013; Dalal, 2005; Viswesvaran \& Ones, 2002). Overall, the extant literature suggests subordinate's interactional justice helps shape the effects of abusive supervision on their performance. 
H4: Interactional justice mediates the relationship between AS and (a) Task

Performance, (b) OCBO, (c) OCBI, (d) CWBO, (e) CWBI.

\section{Organization-referenced Attitudes Linking AS to Job Performance}

\section{Perceived Organizational Support}

Central to organizational support theory (Eisenberger, 1986) are the ideas that employees tend to personify (assign humanlike qualities to) their organization and view the motives of its agents (decision-makers) as those of the organization proper. Perceived organizational support (POS) refers to employees' "global beliefs concerning the extent to which the organization values their contributions and cares about their well-being" (Rhoades \& Eisenberger, 2002, p. 698). Abused subordinates are likely to infer that their employer, having placed the supervisor in a position of formal authority and failed to take corrective action, cares little about them (low POS). Low POS should in turn reduce subordinate task performance and OCBs and increase CWBs.

Relative to the other mediators under present investigation, there is less direct empirical evidence supporting the mediating role of POS — and the strength of the indirect effect appears to be moderated by how much blame subordinates assign to the organization. For instance, Bowling and Michel (2011) found that subordinates who attributed abusive supervision to the organization were more likely engage in CWBOs (Bowling \& Michel, 2011). Moreover, POS was found to mediate the relationship between abusive supervision and task performance, $\mathrm{OCBs}$ and $\mathrm{CWBs}$ - but the indirect effect was observed only among persons who viewed their supervisor as highly embodying the organization (Shoss, Eisenberger, Restubog, \& Zagencyzk, 2013). In addition, prior meta-analytic studies link 
abusive supervision to POS (Mackey et al., 2017) and link POS to in-role performance (task performance) and extra-role performance (OCBs; Rhoades \& Eisenberger, 2002).

H5: Perceived Organizational Support mediates the relationship between AS and (a) Task Performance, (b) OCBO, (c) OCBI, (d) CWBO, (e) CWBI.

\section{Affective Organizational Commitment}

Organizational commitment theory (Allen \& Meyer, 1990) proposes three components to explain why employees stay with an organization: (1) Affective commitment (desire to remain with organization), (2) Continuance commitment (unwillingness to forfeit side-bets associated with leaving), and (3) Normative commitment (sense of loyalty and obligation to organization; Allen \& Meyer, 1990). Consistent with the idea that some subordinates blame the organization for their abusive supervisors are observations of a negative association between abusive supervision and subordinates' affective attachment to the organization (Duffy et al., 2002; Tepper, 2000; Tepper, Henle, Lambert, Giacalone, \& Duffy, 2008). Moreover, affective commitment is negatively associated with task performance and OCBs (Cetin, Gurbuz, \& Sert, 2015; Riketta, 2002).

Researchers have observed the mediating role of affective commitment in the relationship between abusive supervision and (1) task performance (Yu, Lin, Wang, Ma, Wei, et al., 2016), and (2) CWBOs (Tepper et al., 2008). On the other hand, the mediating role of affective commitment in the abusive supervision- OCB relationship has not been previously reported. The indirect effects of abuse on OCBs (via affective commitment) can be inferred from meta-analytic evidence of a positive relationship between (1) satisfaction with one's supervision and affective commitment (Meyer et al., 2002) and (2) affective commitment and OCBs (Cetin et al., 2015; Riketta, 2002). The negative effects of abusive 
supervision on employees' desire to remain with the organization should in turn reduce their willingness to engage in discretionary acts beneficial to the organization. Indeed, employees' limited discretion over task performance relative to contextual performance suggests the indirect effects of abuse (via affective commitment) should more pronounced for OCBs and CWBs than for task performance.

H6: Affective Organizational Commitment mediates the relationship between AS and (a) Task Performance, (b) OCBO, (c) OCBI, (d) CWBO, (e) CWBI.

\section{Competing Mechanisms}

It is not my intention to propose yet another mechanism linking abusive supervision to job performance, as I believe each of the mechanisms presented above likely explains a portion of the overall variance in employee job performance. Instead, the primary contribution of this study is to evaluate the relative importance (i.e., proportion of variance explained) of these mechanisms in a single sample. That said, I perform a simultaneous test of the mediators discussed above to investigate how much each contributes to the relationship between abusive supervision and job performance when they are all considered. Until now, the abusive supervision literature features few empirical investigations that include multiple mediators, and fewer still that have used a general sample of employees. I leverage the wide reach of Amazon's Mechanical Turk (MTurk) to recruit a large sample of full-time and part-time workers located in the United States.

MTurk is an online crowdsourcing platform developed and operated by Amazon.com, which claims to have over 500,000 registered workers across 190 countries (Amazon.com, 2016). The term crowdsourcing — a blend of "crowd" and "out-sourcing"- 
refers to the procurement of information and/or services from a collection of individuals (Lovett, Bajaba, Lovett, \& Simmering, 2018). Arguably, much of the popularity of MTurk lies in the platform's ability to remove logistical challenges regarding the procurement of human labor across a geographically dispersed group of individuals. In addition, MTurk protects user privacy by employing a set of unique - yet anonymous - identifiers which allow human resources to be managed without the need to reveal the identity of its users.

Due to their temporal immediacy, affective mediators (PA, NA) should have a stronger indirect effect on the relationship between abusive supervision and the performance outcomes of interest. Moreover, because abusive supervision involves interactions with the supervision, supervisor-directed attitudes should have stronger indirect effects than organization-related attitudes. The following hypotheses formalize these general expectations.

H7: PA has a stronger indirect effect than AOC in the relationship between AS and (a) Task Performance, (b) OCBO, (c) OCBI, (d) CWBO, (c) CWBI. H8: PA has a stronger indirect effect than POS in the relationship between AS and (a) Task Performance, (b) OCBO, (c) OCBI, (d) CWBO, (e) CWBI. H9: PA has a stronger indirect effect than LMX in the relationship between AS and (a) Task Performance, (b) OCBO, (c) OCBI, (d) CWBO, (e) CWBI. H10: PA has a stronger indirect effect than IJ in the relationship between AS and (a) Task Performance, (b) OCBO, (c) OCBI, (d) CWBO, (e) CWBI. H11: NA has a stronger indirect effect than $A O C$ in the relationship between AS and (a) Task Performance, (b) OCBO, (c) OCBI, (d) CWBO, (c) CWBI. 
H12: NA has a stronger indirect effect than POS in the relationship between AS and (a) Task Performance, (b) OCBO, (c) OCBI, (d) CWBO, (e) CWBI. H13: NA has a stronger indirect effect than LMX in the relationship between AS and (a) Task Performance, (b) OCBO, (c) OCBI, (d) CWBO, (e) CWBI.

H14: NA has a stronger indirect effect than IJ in the relationship between AS and (a) Task Performance, (b) OCBO, (c) OCBI, (d) CWBO, (e) CWBI. H15: AOC has a stronger indirect effect than LMX in the relationship between AS and (a) Task Performance, (b) OCBO, (c) OCBI, (d) CWBO, (c) CWBI.

H16: AOC has a stronger indirect effect than IJ in the relationship between AS and (a) Task Performance, (b) OCBO, (c) OCBI, (d) CWBO, (e) CWBI. H17: POS has a stronger indirect effect than LMX in the relationship between AS and (a) Task Performance, (b) OCBO, (c) OCBI, (d) CWBO, (e) CWBI.

H18: POS has a stronger indirect effect than IJ in the relationship between AS and (a) Task Performance, (b) OCBO, (c) OCBI, (d) CWBO, (e) CWBI.

\section{Ethical Organizational Climate}

Organizational climate research often rests on the assumption that individuals develop summary perceptions of the organization based on their observation of the work environment and organizational practices and procedures (Schneider, 1975). Schneider and Reichers (1983) identify three sources of organizational climate, which broadly refers to descriptions of the work environment that are shared among organizational members. 
Specifically, they propose that organizational climates emerge from (1) observations of the physical environment (structural), (2) organizational processes such as hiring and selection, and (3) interactions that occur during socialization. That is, organizational members share similar perceptions of organizational events because (1) they are objective observers and actually share a similar environment, (2) organizational processes have resulted in an organization whose members are similar and thus judge the environment in similar ways (organizational processes; i.e., Attraction-Selection-Attrition framework), and (3) social interactions during newcomers' socialization period result in them adopting the views of the organization held by the existing membership.

Researchers have identified a variety of organizational climates-including innovation climates (Klein \& Sorra, 1996), safety climates (Zohar, 2010), and justice climates (Liao \& Rupp, 2005) — and have shown that these climates are associated with important organizational outcomes. In addition, meta-analysis of the antecedents of abusive supervision has shown a positive relationship (mean $r=.38, k=7$ ) between organizational climates that promote aggressive norms and abusive supervision (Zhang \& Bednall, 2016).

\section{Ethical Climate Theory}

ECT (Victor \& Cullen, 1987) draws from Kohlberg's (1984) work on moral development and Schneider's (1975) work on organizational work groups- to propose a two-dimensional model of EOC types (Simha \& Cullen, 2012). The first dimension is ethical philosophy. Kohlberg (1984) argued that as individuals develop, they progress through distinct stages of moral reasoning. Stages of moral reasoning have been shown in 
prior research to be incompatible with each other, suggesting that true qualitative differences exist among the various stages (Cullen, Victor, \& Bronson, 1993).

Whereas initial stages of moral reasoning are governed by concerns for rewards and the fear of punishment (egoism), later stages are governed by concern for maximizing joint rewards (utilitarianism) and by concerns related to one's moral obligations to society (deontology). Cullen et al., (1993) argue that, "organizations, like individuals, may be characterized as instrumental, caring, or principled" (p. 668), which correspond to the ethical philosophies of egoism, utilitarianism, and deontology, respectively. This argument is consistent with research showing that individuals ascribe personal qualities to organizations (Eisenberger et al., 2010).

Drawing upon Schneider's (1983) work, ECT also proposes that ethical criteria are considered at three levels-individual, local, and cosmopolitan-corresponding to the three referent groups used in ethical decision-making, the self, the organization, and society, respectively (Martin \& Cullen, 2006). At the individual level, ethical criteria are considered in relation to the self- and interpersonal relationships involving the self (i.e., friendships). At the local level of analysis, ethical criteria focus on groups within the social system (team, department, organization). Finally, at the cosmopolitan level of analysis, the referent group is broad and may include humanity and/or society at large.

ECT crosses three ethical philosophies (egoism, utilitarianism, deontology) with three loci of analysis to hypothesize nine possible EOC types. When ethical criteria are characterized by egoism, a focus on the self (i.e., individual locus of analysis) creates an EOC of self-interest. Organizational members are viewed as making ethical decisions in ways that maximize their own gains, even at the expense of others (Martin \& Cullen, 2006). 
The same ethical philosophy (egoism) yield EOCs based on company profit and efficiency when analyzed at the local and cosmopolitan levels, respectively.

When ethical criteria are characterized by concern for the well-being of others (i.e., benevolence), a focus on the individual locus of analysis creates an EOC of friendship. An ethical philosophy of utilitarianism yields a climate of team interest when analysis takes local level and a climate of social responsibility when analysis takes a cosmopolitan level. Utilitarianism views the maximization of utility (i.e., hypothetical sum of all parties' wellbeings minus sum of all parties' suffering) as the primary determinant of whether a given behavior is ethical. Finally, an ethical philosophy of principle (i.e., deontology) results in the EOCs of independence, rules, and law-and-code at the individual, local, and cosmopolitan locus of analysis, respectively.

Deontology emphasizes individual's sense of duty and obligation. In contrast to philosophies of egoism and utilitarianism, deontology emphasizes the process over the outcome (i.e., means justify ends). That is, egoism and utilitarianism are concerned with whether behaviors result in the maximization of self-interest (egoism) or in the maximization of the interests of all involved (utilitarianism). Deontology on the other hand, is concerned with whether the process applied to judge ethical dilemmas adheres to external codes. Behaviors are ethical when they conform with external codes and unethical when they do not.

Empirical research has confirmed several predictions from ECT (Simha \& Cullen, 2012). In a study of salespersons, Barnet and Vaicy (2000) found that EOC moderated the relationship between behavioral intentions to engage in unethical behavior (questionable sales practices) and actual engagement in unethical behavior such that it was weaker for 
employees who reported an EOC of benevolence (and marginally weaker for those who reported a principle climate). Moreover, an EOC of egoism seems to enhance the relationship between unethical intentions and unethical behaviors, although the effect was not significant (Barnet \& Vaicy, 2000).

ECT research suggest that in addition to behavioral intentions, EOCs influence employee reactions to work events. For instance, studies have linked EOCs to employee attitudes towards their organization, attitudes towards the supervisor, and to in-role and extra-role performance (Bollman \& Krings, 2016; Bulutlar \& Oz, 2009; Cullen et al., 2003; DeConinck, 2011; Hsieh \& Wang, 2016; Leung, 2008; Mulki, Jaramillo, \& Locander, 2006; Schwepker, 2001; Vardi, 2001). In addition, Mayer, Kuenzi, and Greenbaum's (2009) review of the EOC literature outlines significant evidence linking EOCs to workrelated attitudes.

Bulutlar and Oz (2009) reported a positive relationship between interpersonal mistreatment (bullying) and EOCs marked by egoism in a sample of full-time employees. However, their study did not discern across sources of the bullying, making it impossible to isolate the relationship between EOC and supervisory mistreatment. Moreover, a study by Brown, Treviño, and Harrison (2005) investigated the role of leaders in shaping EOC. They distinguished between ethical leadership and related leadership concepts (e.g., transformational leadership) and noted that abusive supervision "contrast sharply with the care and concern ethical leaders display" (p. 122). Consistent with this reasoning, they observed a strong negative correlation $(r=-.61)$ between ethical leadership and abusive supervision in a sample of working MBA students (Brown et al., 2005; Study 5). 
Despite the strength of this relationship, Brown et al. (2005) concluded that abusive supervision and ethical leadership were conceptually distinct. Moreover, Detert et al. (2007) showed that abusive supervision but not ethical leadership predicted unit-level food theft (i.e., counterproductivity). A recent study by Lin, Ma, and Johnson (2016) further suggests that the relationship between abusive supervision and ethical leadership depends on whether a within-subject approach is taken. Lin et al. (2016) assessed leaders four times over a period of three days and observed that behaving ethically was associated with an increase in abusive supervision on the following day. Results were consistent across two samples suggest that the relationship between abusive supervision and ethical leadership is mediated by supervisor's cognitive resource depletion and moral licensing (i.e., "moral credits" accumulated from earlier displays of ethical behavior allow actor to behave unethically without loss of status). Ironically, a leader's attempt to behave ethically may produce - at least in the short-run - higher levels of abusive supervision.

In addition, numerous investigations (e.g., Grojean, Resick, Dickson, \& Smith, 2004; Neubert, Carlson, Kacmar, Roberts \& Chonko, 2009; Wimbush \& Shepard, 1994) show that supervisors (1) play a unique role in establishing EOCs and (2) are a source of employees' EOC perceptions (Martin \& Cullen, 2006). Zhang and Bednall's (2016) metaanalysis yielded a similar conclusion. Specifically, they report a strong negative association (mean $r=-.57 ; k=7$ ) between abusive supervision and ethical leadership and a strong positive association (mean $r=.58, k=10$ ) between abusive supervision and unethical leadership. Although studies that investigate ethical leadership have yielded interesting insights, the impact of EOC has yet to be investigated in the context of abusive supervision. 
Employee's perceived ethical climate (PEC) reflects "holistic impressions of the organizations' ethical context” (Hsieh \& Wang, 2016, p. 3602). Hsieh and Wang (2016) measured PEC using a combination of the five empirically derived EOC types (instrumental, caring, independence, rules, and law-and- code). Instrumental EOC items were reverse-scored. Fit indices from a confirmatory factor analysis for five first order factors and one second-order factor were within an acceptable range—which the authors took as evidence that PEC formed a global construct. Although taking this measurement approach runs the risk of ignoring important differences across the EOC types predicted by ECT, it is pragmatic given the present state of the EOC literature and the focus on employee outcomes. In a recent review of the research related to the effects of EOC on organizational outcomes, Simha and Cullen (2012) note the following:

The essential theme emerging from this stream of research is that benevolent and principled climates (i.e., caring, independence, rules, and law and code) are the climates associated with positive outcomes, and egoistic climates (i.e., instrumental) are associated with a whole host of negative outcomes (p. 27).

In light of these observations, PEC reflects an adequate means of conceptualizing the ethical climate perceptions. To my knowledge, no prior study has analyzed the relationship between abusive supervision and EOC (or PEC). A study that comes close however, Biron (2010), reports a negative correlation $(\mathrm{r}=-.25)$ between perceived organizational ethical values (ethical orientation of organizational members) and perceptions of abusive supervision. In addition, many of the hostile behaviors that characterize abusive supervision are, arguably, more tolerated egoistic EOCs (i.e., low PEC) than under benevolent or principled EOCs (i.e., high PEC). PEC is therefore expected to have a negative relationship with abusive supervision. 
In contrast to the numerous studies linking ethical climate to organizationreferenced attitudes, direct empirical support for the relationship between ethical climate and the supervisor-referenced attitudes considered in this study (LMX, IJ) is lacking. Nonetheless, two prior studies (e.g., DeConinck, 2011; Mulki, Jaramillo, \& Locander, 2006) observe a strong positive correlation between ethical norms and supervisory trusta key element of LMX (see Gerstner \& Day, 1997). These studies suggest that EOCs that emphasize concern for others (e.g., Benevolence, Principle) are more likely to promote fair interpersonal treatment among organizational members than EOCs that emphasize personal gains. Thus, PEC should have a positive association with LMX and IJ.

Finally, extensive empirical evidence links EOC to work-related attitudes that reference the organization (for reviews see Arnaud \& Schminke, 2012; Martin \& Cullen, 2006; Simha \& Cullen, 2012). Results from several investigations (e.g., Ambrose et al., 2008; Cullen et al., 2003; Neubert et al., 2009; Schwepker, 2001) on the effects of EOC on organizational commitment suggest that EOCs dominated by an egoism philosophy reduce while benevolent and principled climates promote organizational commitment. Overall, the results of several studies suggest a positive association between an organization's ethical context and work-related attitudes that reference the organization (e.g., AOC; DeConinck, 2011, Hsieh \& Wang, 2016; Neubert et al., 2009; Parboteeah et al., 2003), and lead to the following expectations:

H19: PEC has a negative association with (a) AS and a positive association with (b) LMX, (c) IJ, (d) AOC, and (e) POS. 


\section{Moderating Role of PEC}

Prior research (Eisenberger et al., 1997) indicates that employees view their organization as having control over their relationship with their supervisor. Indeed, the duties and functions of the supervisor (e.g., staffing, discipline) lead employees to view them as organizational agents and to see their actions as representative of the organization proper (i.e., high SOE; Eisenberger et al., 2010). In addition, Wang and Jiang (2015) report that abusive supervision has a negative impact on IJ only when organizational attributions for abusive supervision is low (vs. high; indicating that supervisors received greater blame).

It is proposed that PEC affects how subordinates assign blame for the abuse. When PEC is low, subordinates may view their organizations as promoting (or at the very least failing to sanction) ethical decision making that regards self-interests and personal gain as the primary criteria. Abusive supervision in this context-so long as it benefits the supervisor-would not be inconsistent with the values of the organization may allow supervisors to "save face" at the expense of the organization. Stated differently, employees are likely to place a greater share of the blame for the abuse on the supervisor at high levels of PEC (i.e., climates characterized by greater benevolence).

Among employees who have these beliefs regarding the ethical decision making of their fellow organizational members, abusive supervision may simply reflect "the way things are done around here." In contrast, when employees perceive that their firm's EOC espouses a concern for the well-being of others and rejects self-interested behaviors (high PEC), the hostile actions of their supervisors are less likely to be viewed as being aligned 
with the organization's ethical values and/or as reflecting the organization's motives and intentions.

In other words, increasing levels of PEC should correspond with supervisors being ascribed a greater share of the blame for their hostile actions. In the present study, this shift in blame and responsibility for the hostility is reflected by pattern of interactions between abusive supervision and PEC such that the (negative) relationships between abusive supervision and attitudes that reference the supervisor (LMX, IJ) are stronger when PEC is high as opposed to low, while the negative relationships between abusive supervision and attitudes that reference the organization (POS, AOC) are stronger when PEC is low as opposed to high.

H20: PEC moderates the relationship between abusive supervision and (a)

LMX and (b) IJ, such that each is stronger when PEC is HIGH (vs LOW).

H21: PEC moderates the relationship between abusive supervision and (a) POS and (b) AOC, such that each is stronger when PEC is LOW (vs HIGH).

\section{Chapter Summary}

The research reviewed clearly demonstrates a link between abusive supervision three aspects of subordinate's job performance - task performance, OCBs, and CWBs. The relative contribution of affective (PA, NA) and attitudinal (LMX, IJ, POS, AOC) mediators

of the relationship between abusive supervision and job performance is presently investigated. Evidence supporting each mechanism, which can be found in prior theoretical work and in empirical investigations, is reviewed. Finally, the concepts of EOC and PECwhich reflects employees' holistic assessment of their organization's ethical context—are discussed and prediction are made regarding the association between PEC and the work- 
related attitudes (LMX, IJ, POS, AOC) framed as mediators of abusive supervisionsubordinate job performance relationships. The chapter concludes with a discussion regarding the moderating role of PEC in the relationship between abusive supervision and these same work-related attitudes. 


\section{METHOD}

\section{Recruitment and Eligibility Criteria}

The present study was described to prospective participants as a "Survey of workplace experiences," and was made visible only to persons residing within in the United States. The posting also contained privacy disclosures that explicitly guaranteed the confidentiality of participant data. The length to complete the questionnaires was stated as 20 and 10 minutes for the participant and coworker questionnaires, respectively. These estimates are based on a reasonable pace of 4 items per minute and total item counts of 86 (participant survey) and 34 (coworker survey). Moreover, the posting stated that individuals whom are invited to participate must meet the conditions listed. Eligibility status was determined through responses to the demographic items appearing at the beginning of the questionnaires.

To be included in the study, focal employees had to meet the following eligibility criteria (listed conspicuously within the study posting). Specifically, at the time of the study, he or she must have been (1) gainfully employed (not volunteering or interning), (2) working 20 or more hours per week (on average), (3) working for the same organization for at least one year, (4) working under the same (and current) supervisor for at least the prior six months, and (5) willing to invite one or more eligible coworkers to the study. As noted in the posting, coworkers are eligible to participate if they (1) work at the same organization as the focal participant, (2) have contact with the focal participant on a consistent basis (at least once per week) and are at least moderately familiar with the focal participant's formal job duties and obligations. In addition, all study participants had to be 18 years or older. 
In both the focal employee and coworker questionnaires, the demographic items are presented first in order to determine participant eligibility. In the focal employee questionnaire, the demographic items are followed by instructions on how to invite one or more coworkers to the study. Upon meeting eligibility criteria, participants are presented with the abusive supervision scale, the PEC instrument, and the state affect and attitude measures. On the coworker's questionnaire, self-rated abusive supervision and PEC are presented immediately after the demographic questionnaire, and this is followed by the task and contextual job performance measures, which are presented in a random order to reduce concerns of order effects.

\section{Participants and Procedure}

Approval for the recruitment and research protocol was obtained from the author's university research ethics review board prior to the start of recruitment efforts. Participant recruitment efforts are conducted on Mechanical Turk (MTurk) and limited to adult participants whom are US residents. The MTurk platform caters to two types of usersrequesters and workers. The latter group can browse this "labor marketplace" and select among the posted tasks those they wish to complete - most often remotely-in exchange for cash payment. Although not designed as a research participant management pool, researchers can assume the role of "requesters" and post tasks (e.g., questionnaires, experiments, opinion polls) for "workers" to complete.

Participants were screened out based on their responses to the demographic questionnaire, questionnaire completion times, and/or their failure to provide the correct response to the attention check items. Recruitment proceeded until data was collected from over 250 coworker dyads. Interested participants received a link to the survey landing page 
(hosted on Qualtrics.com), which displayed the adult informed consent form approved by the university's research ethics board.

In accordance with the protocol approved by the ethics board, viewing any section of the questionnaire (including the eligibility section) required each individual (focal employee, coworker) to provide their informed consent. Focal employees were asked to invite one or more eligible coworkers by providing them with a Study Link (survey URL/QR code) and a unique (randomly generated) Invitation Code. The Study Link led interested coworkers to the informed consent landing page of the coworker questionnaire. The Invitation Code allowed the focal participant and coworker questionnaires to be matched.

\section{Measures}

\section{Focal Employee Questionnaire}

In addition to demographic items, the employee's questionnaire contains measures of abusive supervision, perceived ethical climate, positive affective states, negative affective states, leader-member exchange, interactional justice, perceived organizational support, and affective organizational commitment. Unless otherwise stated, response alternatives range from 1 (Strongly disagree) to 5 (Strongly agree). The focal employee and coworker questionnaires appear in Appendices A and B, respectively.

Demographic Items. Focal participants are asked to provide their age (in years), gender, ethnicity, educational achievement (highest degree earned), and location of employment (State). Another set of items inquire about participant's employment, including employment status (part-time, full-time, self-employed, unemployed), whether the participant reports to someone whom they would consider a boss or supervisor, their 
tenure with current organization, tenure at current position/job classification, and the number of hours worked per week (on average).

Participants are also asked to self-identify their job industry sector using the North American Industry Classification System (NAICS; Bureau of Labor Statistics, 2018), which has three levels comprising ten super-sectors (e.g., Natural Resources and Mining), twenty sectors (e.g., Agriculture, Forestry, Fishing and Hunting, Mining, Quarrying, Oil and Gas Extraction) and over 100 sub-sectors (e.g., Crop Production, Animal Production, Forestry and Logging, Fishing, Hunting and Trapping). Finally, participants were provided with a list of common job activities sourced from the Occupational Network $\left(\mathrm{O}^{*}\right.$ NET, 2018), and had to check whether each of the 40 generalized work activities listed (e.g., Getting Information, Developing Objectives and Strategies, Interacting with Computers, etc.) applied to them.

Abusive Supervision. Abusive supervision is measured using 15 items from Tepper's (2000) scale. Respondents are asked to rate the frequency of abusive supervisory behaviors in the prior 30 days. Response alternatives range from 0 (Never) to 5 (Very often). Sample items include "ridicules me", "invades my privacy," and "makes negative comments about me to others." Higher scores are interpreted as more frequent abuse.

Perceived Ethical Climate. PEC is measured using Hsieh and Wang's (2016) PEC instrument, which borrows items from the five ethical climate subscales developed by Victor and Cullen (1988). Specifically (1) six items are used to measure Instrumental climate, (2) four items are used to measure Rules climate, (3) four items are used to measure Law and Code climate, (4) four items are used to measure Caring climate, and (5) four items are used to measure an Independence climate. Instrumental climate scores are 
reversed and added to the sum of the remaining four subscales to arrive at an overall score for PEC.

Positive Affective States. PA is measured using 10 items from van Katwyk, Fox, and Spector's (1999) job-related affective well-being scale (JAWS). Respondents are asked to indicate how often any part of their job has made them feel each target emotion during the prior 30 days. Responses alternatives range from 1 (Never) to 5 (Extremely often). Sample items include "at ease," "calm," and "content." Higher scores indicate more frequently experienced PA.

Negative Affective States. NA is measured using 10 items from van Katwyk, et al.'s (1999) job-related affective well-being scale (JAWS). Respondents are asked to indicate how often any part of their job has made them feel each target emotion during the prior 30 days. Responses alternatives range from 1 (Never) to 5 (Extremely often). Sample items include "angry," "anxious," and "discouraged." Higher scores indicate more frequently experienced PA.

Leader-Member Exchange. LMX is measured using seven items from the LMX7 (Graen \& Uhl-Bien, 1995). Response alternatives vary across items and range from 1 to 5. The word leader in each item is substituted for the word "supervisor" for consistency. For instance, the item, "How well does your supervisor understand your job problems and needs?", has the following response alternatives: (1) Not a bit, (2) A little, (3) A fair amount, (4) Quite a bit, and (5) A great deal. The very next item, "How well does your supervisor recognize your potential?", has the following response alternatives: (1) Not at all, (2) A little, (3) Moderately, (4) Mostly, (5) Fully. The wording and anchoring of each item are such that higher ratings are invariably associated with higher LMX. 
Interactional Justice. IJ is measured using Moorman's (1991) six-item scale. Sample items include, "Your supervisor considered your viewpoint," and "Your supervisor took steps to deal with you in a truthful manner." Higher scores correspond to higher IJ.

Perceived Organizational Support. POS was assessed using eight items from the Survey of Perceived Organizational Support (SPOS; Eisenberger, Cummings, Armeli, \& Lynch, 1997). Sample items include, "My organization would forgive an honest mistake on my part", and "My organization really cares about my well-being." Higher scores indicate greater POS.

Affective Organizational Commitment. AOC is measured with eight items from the Affective Commitment Scale (Allen \& Meyer, 1990). Sample items include, "I enjoy discussing about my organization with people outside it," and "This organization has a great deal of personal meaning for me." Higher scores on the ACS indicate greater AOC.

\section{Coworker Questionnaire}

The coworker's questionnaire will contain the same demographic items as the employee survey (see above) with the addition of three items - the first two of which shall be used to determine coworker eligibility. The first asks the coworker to rate how frequently they work with the target employee (i.e., share the same shift). Response alternatives are anchored as follows: (1) less than once per week, (2) once per week, (3) two or more times per week, and (4) every shift. The second asks coworkers to rate the extent to which he or she is familiar with the formal duties and responsibilities of the target participant's job. Response alternative are: (1) Not at all familiar, (2) Somewhat familiar, (3) Very familiar, and (4) Extremely familiar. To be eligible, coworkers must respond with 3 or higher to both items. 
Finally, the third additional demographic item asks coworker respondents to rate how often he or she has had the opportunity to observe the work behaviors of the target employee during the prior 30 days. Response alternatives are anchored as follows: (1) Never, (2) Less than once per shift, (3) Once per shift, and (4) Two or more times per shift. Confidentiality and privacy notices are prominently displayed above the instructions, which clearly state that participants are to refer to the target employee when responding to each item. All items are rated on the same frequency scale — which ranges from 1 (Never) to 5 (Extremely often).

Coworker-rated Abusive Supervision. This instrument was adapted from Tepper's (2000) 15-item self-report Abusive Supervision Scale described above. Items were framed for coworkers. For instance, coworkers were asked how often they have witnessed the focal employee's supervisor express each target behavior (e.g., was ridiculed, invaded their privacy, did not give them credit for jobs requiring a lot of effort, etc.) towards the focal employee (i.e., the coworker that invited them to participate). The frequency scale used in the original measure was retained.

Task Performance. Task performance is measured using four items from Williams and Anderson's (1991) In-Role Performance Scale. Respondents rate how often their coworker "Meets formal performance requirements of the job." Higher scores indicate more frequent engagement in behaviors associated with high task performance.

Organizational Citizenship Behaviors. OCBs are measured using Fox, Spector, Goh, Bruursema, and Kessler's (2009) scale, which includes six items that measure OCBI (e.g., "Helps a less capable co-worker lift a heavy box or other object.") and six items that 
measure OCBO (e.g., "Volunteers for extra work assignments."). Higher scores indicate more frequent OCBs.

Counterproductive Work Behaviors. CWBs are measured using Bennett and Robinson's (2000) Workplace Deviance Scale, which includes seven items to measure CWBI (e.g., "Says something hurtful to someone at work.") and twelve items to measure CWBO (e.g., "Puts little effort into his or her work."). The wording of items was adapted for coworker report. For instance, "Littered your work environment" was changed to "Litters your work environment" One item, "Falsified receipts to get reimburse for more money than you spent on business expenses" was dropped because it is very narrow in scope (i.e., applicable only to select jobs) and has a very low endorsement rate (Bennet \& Robinson, 2000). Higher scores indicate more frequent CWBs.

\section{Data Integrity Checks}

The present protocol (i.e., remote un-proctored administration) poses concerns regarding data quality. Relative to "standardized" laboratory conditions, the environment/setting of participants completing a web-based questionnaire that is mobileenabled (i.e., functional on devices with smaller screens) may vary considerably. The potential for distractions during administration can also be a source of careless responding and a cause for validity concerns. To detect insufficient effort and careless (i.e., inattentive, low effort) responding, I measure completion times and include instructed-response ("attention check") items scattered throughout the questionnaires. Participants with survey completion times under 400 seconds (or 240 seconds for coworker questionnaire) are removed from the dataset. 
Inattentive responders are detected using a set of four (two for the coworker questionnaire) attention check items, a strategy that has been shown to be effective without compromising scale validity (Kung, Kwok, \& Brown, 2018). In addition, these items have a relatively straightforward interpretation. Consider the following attention check item, "Please choose strongly agree." It is reasonable to assume that responses to this item other than "strongly agree" is evidence that the participant is responding carelessly and/or randomly. Data from respondents that fail more than one attention check item (or any attention check item in coworker questionnaire) is discarded.

The full employee sample ("full dataset") is contrasted with the usable sample (i.e., containing only matched dyads) to assess the extent to which the two groups vary across key variables (demographic and substantive). According to Cohen's (1988) classification of effect sizes for the social sciences, values between .1 and .23 are considered small, values between .24 and .36 are considered moderate (medium), and values greater than .36 suggest a large effect. Note that values below .1 are considered negligible. Hedge's $g$ statistic is used to estimate the effect of matching on each continuous variable. The computation and interpretation of values for Hedge's $g$ is performed in much the same way as for Cohen's $d$.

In contrast to Cohen's $d$ however, the formula for Hedge's $g$ allows for the pooled standard deviation (Eq. 1 denominator) to be weighed by sample size. Since the unmatched group is expected to be more than 10 times greater than the matched group, it seems appropriate to reflect this reality by computing the pooled standard deviation using sample size weighed SDs. Thus, Hedge's g is computed shown in Equation 1 below, where 
$\bar{y}_{\text {Matched }}, n_{\text {Matched }}$, and $s_{\text {Matched }}$ refer to the mean, sample size, and standard deviation of the matched group, respectively.

$$
g=\frac{\bar{y}_{\text {Unmatched }}-\bar{y}_{\text {Matched }}}{\sqrt{\frac{\left(n_{\text {Unmatche }}-1\right) s_{\text {Unmatched }}^{2}+\left(n_{\text {Matched }}-1\right) s_{\text {Matched }}^{2}}{\left(n_{\text {Unmatche }}-1\right)+\left(n_{\text {Match }}-1\right)}}}
$$

\section{Analysis of Normality}

The characteristics of the distribution of the sample data (e.g., skewness, kurtosis) affect many types of hypothesis tests, including tests of (difference between) means, variance, and covariance (Mardia, Kent, Bibby, 1979). Although univariate normality may be evaluated by visual means (e.g., graph of Quantile-Quantile plots), two distinct, albeit related numerical indicators - skewness and kurtosis - may be tested statistically (Cain, Zhan, \& Yuan, 2016). Skewness is a measure of the symmetry of the distribution. Kurtosis roughly measures the "tailedness" (i.e., mass of tails) of the distribution (DeCarlo, 1997).

Normal distributions are symmetrical (skewness $=0$ ) and have a kurtosis of 3 (DeCarlo, 1997). Deviations from normality at the univariate level is indicated by observed values of skewness (and/or kurtosis) that significantly differ from values that would be expected if the sample data is MVN. The Shapiro-Wilk test (SW; Shapiro \& Wilk, 1965) is a univariate normality test that detects departures from normality due to skewness and/or kurtosis. The $\mathrm{S}-\mathrm{W}$ test has been shown to perform well with simulated data and has higher power than other "mainstream" univariate normality tests (e.g., Kolmogorov-Smirnov Test, Lilliefors Test) for sample sizes under 2000 (Razali \& Wah, 2011). S-W tests are performed on each study variable, including single items and scale means-leading to a total of 133 tests. As recommended by DeCarlo (1997), a Bonferroni correction (alpha/number of variables $=.05 / 133)$ was made to control for Type 1 error. 
Univariate normality is a necessary (but insufficient) condition for demonstrating MVN. Therefore, two formal tests of MVN are provided by Mardia (1970). Mardia's measures contrast the observed skewness (and kurtosis) in a joint distribution comprised of multiple variables against the values of skewness (and kurtosis) expected if the same number of variables had a joint distribution that is indeed MVN (Cain et al., 2016). Mardia's measures of multivariate skew $\left(b_{1}\right)$ and kurtosis $\left(b_{2}\right)$ are computed using the "Mardia" SPSS macro developed by DeCarlo (1997). Values of $b_{1}$ greater than 0 and/or values of $b_{2}$ greater than $p(p+2)$; where $p$ is the number of variables; indicate departure from MVN. Test statistics (e.g., $b_{1 z} ; b_{2 z}$ ) are formed based on their expected distributions (i.e., chi-square distribution with for $b_{1}$; normal distribution for $b_{2}$ ).

\section{Hypothesis Testing}

\section{Mediation Analyses}

Covariance-based structural equation modeling (SEM) is a general framework for testing causal relationships and allows researchers to distinguish between manifest (observed) and latent (unobserved) variables. In the analysis of questionnaire/survey data for instance, constructs of interest (e.g., abusive supervision) can be represented as latent factors and the survey items associated with these constructs (e.g., abuse 1, abuse 2, abuse 2, etc.) as their indicators. Although SEM is an extension of regression analyses, by integrating the techniques of confirmatory factor analysis, it has the advantage of allowing measurement errors to be estimated rather than relying on the assumption that constructs

are measured with perfect reliability (Iacobucci, 2009). Nonetheless, the validity of inferences made with SEM also depend on numerous assumptions (e.g., no misspecification, MVN; Kline, 2011). 
Moreover, SEM has sample size requirements that should be considered a priori. Past research suggests sample size requirements vary as a function of the magnitude of the effects to be detected, the desired power and level of confidence, the complexity of the model (number of latent factors, number of indicators, the ratio of factors to indicators), measurement qualities (factor loadings, scale of measurement), and the distributional properties of the population and sample data (Westland, 2010; Wolf, Harrington, Clark, \& Miller, 2015). In general, sample size requirements increase as the effects to be detected become smaller, the structural model(s) to be tested increase in complexity (number of factors/indicators), and/or the sample data departs from MVN. Finally, the choice of estimator (e.g., maximum-likelihood, least-squares) and the presence of missing data also plays a role in determining minimum sample size (Wolf et al., 2015).

Some widely cited suggestions regarding the minimum sample size required for SEM analyses range from at least 100 (Anderson \& Gerbing, 1984) to 200-400 (Jackson, 2001). It is crucial to note that suggestions in the literature are applicable only to relatively simple models and in situations where the sample data approximates MVN. Indeed, sample size requirements can be several orders of magnitude larger if departures from MVN occur and/or the indicators are not on a continuous scale of measurement (Jöreskog \& Sörbom, 1996). In the present study, an SEM to test the mediation hypotheses would need to include 13 latent factors and a total of 120 indicators measured on ordinal scales.

Assuming no departures from MVN, achieving power of .80 to detect an effect of .15 (at the .05 level of significance) in a model of with 13 latent factors and 120 indicators requires a sample size of at least 850 (Westland, 2010). In contrast, achieving the same statistical power to detect an effect of equal magnitude (at the .05 level of significance) in 
a multiple regression model with seven predictors (six mediators plus abusive supervision) requires only 103 cases (Soper, 2019). If the present sample does not meet the requirements for SEM, hypothesis testing will focus exclusively on manifest variables and proceed using either a normal-theory (if data is MVN) or a nonparametric approach.

Normal Theory. Classical mediation theory (i.e., "causal steps approach"), as outlined by Baron and Kenny (1986), requires the existence of "an effect to be mediated." In other words, the first requirement to test for mediation is the existence of a significant relationship exists between the independent and dependent variable (Path $c^{\prime}$ ). In situations where the direct (Path $c$ ) and indirect effect(s) (Path $a *$ Path $b$ ) are of opposite signs (e.g., competitive mediation) however, Path $c^{\prime}$ (total effect) approaches zero and becomes exceedingly difficult to demonstrate-leading some (e.g., Zhao, Lynch, \& Chen, 2010) to view this initial requirement to test for mediation as inappropriate. The relationships among the Paths a, b, c, and c' are illustrated by the two equations shown below. The total effect of Predictor $\mathrm{X}$ on Outcome $\mathrm{Y}$ is the sum of its direct and indirect effects (Eq. 2). Alternatively, the indirect effect is equal to the difference between the total and direct effects (Eq. 3; MacKinnon, Warsi, \& Dwyer, 1995).

Total effect $($ of $X$ on $Y)=$ path $c^{\prime}=($ path $a *$ path $b)+$ path $c$

Indirect effect $($ of $\mathrm{X}$ on $\mathrm{Y}$, via $\mathrm{M})=$ path $\mathrm{a} *$ path $\mathrm{b}=$ path $\mathrm{c}^{\prime}-$ path $\mathrm{c}$

Accordingly, a variable functions as a mediator when variations in the levels of an independent variable significantly account for variations in the mediator (Path $a$ is significant) and variations in the mediator significantly account for variations in the dependent variable (Path $b$ is significant). Note that an additional requirement to show mediation described by Baron and Kenny - that the previously significant relationship 
between the independent and dependent variable is no longer significant in when Paths $a$ and $b$ are controlled for-is obviated when the initial requirement of a significant total effect is not imposed on the mediation analyses.

If the data is normally distributed (at the univariate and multivariate level), I will test the indirect effect directly using the "product-of-coefficients approach" to test the significance of the indirect effect. The product of coefficients approach requires estimating a set of two regression equations (using ordinary-least-squares; OLS-criterion). In the first equation, the mediator is regressed on the independent variable. In the second equation, the dependent variable is simultaneously regressed on both the independent variable and the mediator. Finally, the indirect effect is tested by estimating the product of the coefficient associated with the predictor (from the first equation) and the coefficient associated with the mediator (from second equation) and dividing this quantity by its standard error to arrive at a critical ratio (Equation 4). It is important to note that this approach assumes the indirect effect $a b$ follows an MVN distribution - an untenable assumption in some situations (MacKinnon, Lockwood, Hoffman, West, \& Sheets, 2002; Preacher \& Hayes, 2004). That said, an alternative approach is followed if the present data does not meet these criteria.

$$
\text { Critical Ratio }=\frac{a b}{s_{a b}}=\frac{a * b}{\sqrt{b^{2} s a^{2}+a^{2} s b^{2}+s a^{2} s b^{2}}}
$$

MacKinnon et al. (1995) found that the standard error estimate used in Equation 4, which adds a third term $\left(\mathrm{sa}^{2 *} \mathrm{sb}^{2}\right)$ to Sobel's (1982) standard error estimator, performed better than standard error estimates that subtracted or omitted the term. In Equation 4, $a$ is the unstandardized regression coefficient associated with the predictor (in the first regression equation), $b$ is the unstandardized regression coefficient associated with the 
mediator (in second regression equation), and $s a$ and $s b$ are their standard errors, respectively. The null hypothesis is rejected if the critical ratio exceeds 1.96 in magnitude (at .05 level of confidence).

Nonparametric Approach. Although MacKinnon et al. found the standard error estimates made using Equation 4 as superior to other "normal theory" estimators of the mediated effect, nonparametric methods are preferred when sample/population distributions deviate from MVN or are unknown (MacKinnon et al., 2002; Preacher \& Hayes, 2004).

Bootstrapping is a nonparametric approach to effect size estimation and theory testing (Preacher \& Hayes, 2004) that, rather than relying on assumptions about the shape of the distribution, requires the analyst to construct an empirical approximation of the distribution of interest (e.g., indirect effect, pairwise contrast) via resampling from the initial sample. Assumptions regarding the distributional properties of the indirect effects (Preacher \& Hayes, 2008) can be relaxed. Once the bootstrap distribution of the effect is obtained through resampling, several methods (e.g., percentile, bias-corrected [BC], accelerated and bias corrected $[\mathrm{BCa}])$ can be used to create bootstrap confidence intervals (Fritz, Taylor, \& MacKinnon, 2012).

In their review of the more widely used methods, Fritz et. al. (2012) concluded that the percentile method had more accurate Type 1 error rates for sample sizes under 2500 than the bias-corrected and accelerated bias-corrected methods. Moreover, recent simulation studies suggest that the, albeit less powerful, percentile method is less prone to elevated Type 1 error rates and therefore the "more widely recommended method for inference about the indirect effect in mediation analyses" (Hayes, 2017, p. 107). 
In the percentile method, the bootstrap distributions that are generated through resampling are sorted in ascending order and values corresponding to the 2.5 th and 97.5 th percentile are selected to create the $95 \%$ bootstrap confidence interval. The null hypothesis is rejected if the interval excludes zero. For instance, to bootstrap the difference between two indirect effects using the percentile method with 1000 resamples, values of this difference are randomly sampled (with replacement) from the current dataset to create one thousand datasets of equal size $(\mathrm{n}=281)$. An estimate of the mean difference is recorded for each dataset and these 1000 estimates-collectively referred to as the bootstrap sampling distribution — are sorted in ascending order. The $25^{\text {th }}$ and $976^{\text {th }}$ values correspond to the lower and upper limits of the $95 \%$ confidence interval, respectively.

Hypotheses regarding competing mechanisms are supported if the two effects differ significantly in magnitude. Specifically, multiple mediator analyses are performed using PROCESS, a free-license computational tool for SPSS developed by Hayes (2012) to facilitate mediation testing for behavioral researchers. PROCESS provides bootstrap standard errors (and confidence intervals) for specific indirect effects, which represent the unique abilities of each mediator to account for the effect of abusive supervision on job performance, controlling for the other mediators in the model (Preacher \& Hayes, 2008).

In addition to providing bootstrapped confidence intervals for specific indirect effects, PROCESS facilitates pairwise contrast between specific indirect effects by providing bootstrap confidence intervals for the difference (or absolute difference, see below) between two specific indirect effects. As Hayes (2017) notes regarding tests involving pairwise contrasts, "a bootstrap confidence interval is derived by estimating the difference between specific indirect effects over repeated bootstrap sampling and model 
estimation" (p. 165). Significant differences between specific indirect effects allows for statistical inferences regarding whether one mechanism is stronger (accounts for more of the effect of $\mathrm{X}$ on $\mathrm{Y}$ ) than another. Five multiple mediator models are estimated, corresponding to the job performance outcomes of interest (task performance, OCBO, OCBI, CWBO, CWBI).

Special care must be taken when the specific indirect effects to be contrasted differ in signs. In these situations, taking the difference between the effects instead of the difference between their absolute values may lead to the false conclusion that one effect is stronger than the other. Consider specific indirect effect $1, a_{1} b_{1}=.10$, and specific indirect effect $2, a_{2} b_{2}=-.10$. Although these values differ by .20 , it would be wrong to conclude that specific indirect effect 1 is stronger than specific indirect effect 2. To avoid these issues, pairwise contrasts focus on differences between the absolute values of specific indirect effects.

If the data is normally distributed, hypotheses involving a single mediator (H1aH6e) are tested using a critical z-ratio test (i.e., Sobel Test; Eq. 4) and supported if the ratio exceed $+/-1.96$. Alternatively, $\mathrm{H} 1-\mathrm{H} 6$ are evaluated using the bootstrapped confidence interval about the indirect effect if data is not normally distributed. Further, $\mathrm{H} 7 \mathrm{a}-\mathrm{H} 18 \mathrm{e}$ are evaluated using multiple mediator models (with all mediators operating in parallel) using PROCESS v3.1 (Model 4; Hayes, 2017) and supported if the bootstrapped confidence interval for the (absolute) difference between the indirect effects excludes zero. Hypothesis 19 (a-e) is are confirmed if PEC has a significant bivariate correlation with AS (negative) and target attitude (positive). 


\section{Moderated Multiple Regression}

The remaining hypotheses (20a-21b) are tested using moderated multiple regression (MMR) and significant interactions are probed at values of PEC equal to plus and minus one standard deviation from the mean, corresponding to high and low values, respectively. Since the current PEC measure is purported to represents a global construct that subsumes five ethical climate types (Hsieh \& Wang, 2016), a confirmatory factor analysis is required to evaluate the dimensional structure of the PEC instrument. Hypotheses 20a-21b are confirmed if (1) interactive effect of abusive supervision and PEC (AS*PEC) significantly predicts each work-related attitude after controlling for the main effects of abusive supervision and PEC and (2) the direction of the interaction is consistent with the stated predictions. 


\section{RESULTS}

\section{Data Cleaning Protocol}

Data was collected between July and September 2018. As shown on Table 3a, a total of 8557 persons agreed to participate in the focal employee survey, from which 6905 completed the eligibility section. I removed 1652 participants did not complete eligibility section and an additional 3613 whom did not meet eligibility criteria. Specifically, one case was removed for age (under 18), 1660 cases were removed because they completed the questionnaire in under 400 seconds, 522 cases were removed for employment status (selfemployed, unemployed, temporary employment), 546 cases were removed for working less than 20 hours per week (on average), 384 were removed because participant indicated they did not have a "boss" or supervisor, and 493 cases were removed because organizational tenure was less than 1 year.

A total of 3292 participants met eligibility criteria. Approximately $17 \%$ of these participants did not provide usable questionnaires, including 554 cases that had multiple sections (i.e., instruments) missing entirely, suggesting the participant quit the questionnaire $(N=543)$. Further, an additional eleven participants were removed because they failed more than one of the four attention check items. The remaining 2738 participants provided usable data.

Data collection began concurrently for focal employees and coworkers. However, the coworker questionnaire remained active for an additional month after the closing of the focal employee questionnaire to allow for the focal participants to successfully recruit a coworker and thereby improve the response rate for matched pairs. As shown on Table 3b, 785 persons agreed to participate in the coworker questionnaire, from which 572 provided 
a valid invitation code. From these, 511 completed the eligibility section and 300 met eligibility criteria. Specifically, four cases were removed for employment status, 29 cases were removed because they completed the questionnaire in under 240 seconds, 10 cases were removed for working less than 20 hours per week (on average), 20 cases were removed for having less than 1 year of organizational tenure, 13 cases were removed because they indicated having contact with the focal participant less than once per week, 24 cases were removed because the participant was less than "Moderately familiar" with the focal employee's job, one case was removed because the participant indicated working outside the US, and 110 cases were removed because coworker indicated their relationship to focal participant was anything other than peer-to-peer (i.e., at the same organizational level; e.g., supervisory relationship, subordinate relationship).

Of the three hundred persons initially eligible to participate, 17 failed one or more of the two attention check items. Although coworker data was collected from 283 focal participants, this included two participants whom each recruited two coworkers. In each case, only the data from the first coworker to complete the questionnaire was retained. Thus, a total of 281 focal participants had a matching coworker whom also provided usable data.

To reiterate, from the 2738 participants who provided usable data, $281(10.3 \%)$ had at least one coworker whom also provided usable data. Two datasets were created. The first is the "full dataset", which includes both matched and unmatched participants and is used to test hypotheses $\mathrm{H} 19-\mathrm{H} 21$ regarding the effects of employee-rated PEC). The second is the "matched-only" dataset, which includes only cases with usable data from both the focal employee and his or her coworker and is used to test hypotheses regarding the indirect 
effects of self-rated abusive supervision on the (focal) employee's job performance, as rated by their peer $(\mathrm{H} 1-\mathrm{H} 18)$.

\section{Description of Sample Data}

Table 4 shows the distribution of participant demographic characteristics and study variables across the full and matched-only dataset for variables measured on continuous scales. Further, Table 5 displays the demographic characteristics across the datasets for categorical/nominal variables. In addition, Table 5 displays data from the Bureau of Labor Statistics (BLS.gov, 2018) showing how these demographic characteristics were distributed across persons employed in the United States (i.e., US labor force) at the time of the study (2018).

\section{Matched-only Dataset}

Participants in the matched-only dataset reported a median age of 32 (Mean $=34$ $\mathrm{SD}=9)$ and $45 \%$ identified as male. Most of this group identifies as Caucasian $(75 \%)$, although a significant portion identify as Asian (7\%), African/Black (9\%), and Hispanic (8\%). The mode response for educational achievement was bachelor's degree (49\%). Moreover, the matched group includes participants from 45 of the 50 US States (90\%) and from all 20 major industrial sectors in the US. Approximately ninety-one percent reported working "full-time", and the average number of hours worked per week was $40(\mathrm{SD}=7)$. This group's average organizational tenure was 6 years $(\mathrm{SD}=5)$.

\section{Full Dataset}

Participants included in the full dataset reported a median age of 31 (Mean $=34$, $\mathrm{SD}=9)$ and $52 \%$ identified as male. Most of this group identifies as Caucasian $(74 \%)$, although a significant portion identify as Asian (8\%), African/Black (16\%), and Hispanic 
(6\%). The mode response for educational attainment was bachelor's degree (45\%). In addition, participants in the full dataset represent all 50 States and all 20 major industrial sectors in the US. Ninety percent reported working "full-time", with an average of 40 hours per week $(\mathrm{SD}=8)$. The average organizational tenure of the unmatched group was 6 years $(\mathrm{SD}=5)$.

Although several study variables show significant mean differences across matched and unmatched groups, the effect size estimates shown in the last column of Table 4 (and discussed below) dispels concerns that these differences are substantial. Moreover, the Pearson correlation coefficients for the study variables appear on Table 6a (unmatched group) and Table $6 \mathrm{~b}$ (matched group). Considering the outcome of the normality analyses (see below), it is prudent to use Spearman's rho correlations as our measure of association. Spearman's rho examines rank order, has same range as the Pearson correlation $(0,1)$, and indicates the strength of a monotonic — but not necessarily linear-association. Tables 7a and $7 \mathrm{~b}$ display Spearman's rho correlations across study variables for the full and matchedonly datasets, respectively.

\section{Comparison of Matched and Unmatched Samples}

The Hedge's $g$ effect size estimates for the difference in means across unmatched and matched groups appear in Table 4. The differences are significant (at the .05 level) for four of the eleven variables. The average effect size across these four variables is .30, which is considered moderate based on Cohen's (1988) classification. Overall, participants in the full dataset tended to report higher levels of PEC, abusive supervision, PA, and NA, as evidenced by the (positive) sign of the effect size estimates. 


\section{Normality Analyses}

The full and matched-only datasets were evaluated for normality at the univariate and multivariate levels. Results of univariate normality $(\mathrm{S}-\mathrm{W})$ tests for the full and matched-only datasets are shown on Tables $8 \mathrm{a}$ and $8 \mathrm{~b}$, respectively. The first column and second columns of Tables $8 \mathrm{a}$ and $8 \mathrm{~b}$ show the name of the variable and the corresponding S-W test statistic, which has values that ranging between 0 and 1 , with values closer to 1 indicating normality (Razali \& Wah, 2011). The third and fourth columns correspond to the degrees of freedom and p-values, respectively, for the S-W statistic. Recall that a Bonferroni correction, which imposes a "penalty" proportional to the number of individual tests performed (i.e., 94 in the full dataset; 133 in the matched-only dataset) is made to control for Type 1 errors.

In the full dataset, the null hypotheses associated with the S-W tests are rejected at the $\mathrm{p}<.0005$ level (Bonferroni-corrected alpha) in all cases, suggesting none of the variables in the matched dataset exhibit univariate normality. MVN tests were performed separately on individual items (86 items) and on scale means ( 8 factors/scales). When the individual items are considered, Mardia's multivariate skew $\left(b_{1}=609.51, \mathrm{p}<.0001\right)$ and multivariate kurtosis $\left(b_{2}=9814.50, \mathrm{p}<.0001\right)$ lead to the rejection of the null hypothesis (at .0001 level) that the data is MVN. Mardia's tests for skew $\left(b_{1}=5.59, \mathrm{p}<.0001\right)$ and kurtosis $\left(b_{2}=59.36, \mathrm{p}<.0001\right)$ also lead to the rejection of the null hypothesis when the set of scale means is considered. These results suggest the full dataset severely departs from MVN.

In the matched-only dataset, the null hypotheses associated with the S-W tests are rejected at the $\mathrm{p}<.0004$ level (Bonferroni-corrected alpha) in all but one case (mean of PA 
items), suggesting none of the variables in the matched dataset exhibit univariate normality. MVN tests were performed separately on individual items (120 items) and on scale means (13 factors/scales). When the individual items are considered, Mardia's multivariate skew $\left(b_{1}=8297.77, \mathrm{p}<.0001\right)$ and multivariate kurtosis $\left(b_{2}=15867.61, \mathrm{p}<.0001\right)$ lead to the rejection of the null hypothesis (at .0001 level) that the data is MVN. Mardia's tests for skew $\left(b_{1}=1847.31, \mathrm{p}<.0001\right)$ and kurtosis $\left(b_{2}=248.38, \mathrm{p}<.0001\right)$ also lead to the rejection of the null hypothesis when the set of scale means is considered. In sum, the data in the matched-only dataset severely departs from MVN.

\section{Mediation Analyses}

The modest sample size $(N=281)$ and distributional properties of the matched-only dataset support the decision to proceed with a manifest variable analysis (rather than covariance-based SEM for hypotheses H1-H18. Overall, it seems unlikely that the present data will yield stable parameter estimates in a structural model involving 13 latent factors and 120 indicators. Therefore, mediation hypotheses are tested using bootstrapped confidence intervals about the indirect effect $(\mathrm{H} 1-\mathrm{H} 6)$ and bootstrapped confidence intervals about the difference between two specific indirect effects (H7-H18). All hypotheses are tested at the .05 level of confidence and all bootstrap standard errors and confidence intervals are based on 5000 samples unless otherwise noted. Moreover, all bootstrap confidence intervals are constructed using the percentile method.

\section{Mediating Effect of Positive Affect}

Tables 9a-9e display a summary of the OLS regression models estimated to test Hypotheses 1a-1e, respectively. These hypotheses reference the mediating effects of PA on each of the five job performance outcomes. The first column shows the name of the path 
in reference. The second and third columns show the unstandardized regression coefficients $(B)$ and their bootstrap standard errors (SE), respectively. The fourth and fifth columns show the lower and upper limits of the $95 \%$ bootstrap confidence interval for the unstandardized coefficients, respectively.

Hypothesis 1a (H1a). As shown on Table 9a, the unstandardized regression coefficient associated with abusive supervision $(B=-0.150)$ is significant in Model A1 and has a bootstrap standard error of 0.078. In addition, the coefficient associated with PA ( $B$ $=$ less than .001) is not significant in Model A2 and has a bootstrap standard error of 0.044 . The product of these coefficients, less than 0.001 , reflects a point estimate of the indirect effect of abusive supervision on task performance via PA and has a bootstrap standard error of 0.008 . The bootstrap confidence interval about the indirect effect has a lower limit of 0.014 and an upper limit of 0.018 , which includes zero. The indirect effect in Model A3 is not significant and thus H1a is not supported.

Hypothesis 1b (H1b). As shown on Table 9b, the unstandardized regression coefficient associated with abusive supervision $(B=-0.150)$ is significant in Model B1 and has a bootstrap standard error of 0.078 . In addition, the coefficient associated with PA ( $B$ $=0.274)$ is significant in Model B2 and has a bootstrap standard error of 0.058 . The product of these coefficients, -0.041 , reflects a point estimate of the indirect effect of abusive supervision on OCBO via PA and has a bootstrap standard error of 0.022 . The bootstrap confidence interval about the indirect effect has a lower limit of -0.090 and an upper limit of -0.004 , which excludes zero. The indirect effect in Model B3 is significant and thus H1b is supported. 
Hypothesis 1c (H1c). As shown on Table 9c, the unstandardized regression coefficient associated with abusive supervision $(B=-0.150)$ is significant in Model $C 1$ and has a bootstrap standard error of 0.079. In addition, the coefficient associated with PA ( $B$ $=0.195$ ) is significant in Model C2 and has a bootstrap standard error of 0.057 . The product of these coefficients, -0.029 , reflects a point estimate of the indirect effect of abusive supervision on OCBI via PA and has a bootstrap standard error of 0.017 . The bootstrap confidence interval about the indirect effect has a lower limit of -0.068 and an upper limit of -0.002 , which excludes zero. The indirect effect in Model $\mathrm{C} 3$ is significant and thus $\mathrm{H} 1 \mathrm{c}$ is supported.

Hypothesis 1d (H1d). As shown on Table 9d, the unstandardized regression coefficient associated with abusive supervision $(B=-0.150)$ is significant in Model D1 and has a bootstrap standard error of 0.077 . In addition, the coefficient associated with PA ( $B$ $=0.149)$ is significant in Model D2 and has a bootstrap standard error of 0.049. The product of these coefficients, -0.022 , reflects a point estimate of the indirect effect of abusive supervision on CWBO via PA and has a bootstrap standard error of 0.014 . The bootstrap confidence interval about the indirect effect has a lower limit of -0.056 and an upper limit of -0.001 , which excludes zero. The indirect effect in Model D3 is significant and thus H1d is supported.

Hypothesis 1e (H1e). As shown on Table 9e, the unstandardized regression coefficient associated with abusive supervision $(B=-0.150)$ is significant in Model E1 and has a bootstrap standard error of 0.080 . In addition, the coefficient associated with PA ( $B$ $=0.157$ ) is significant in Model E2 and has a bootstrap standard error of 0.043 . The product of these coefficients, -0.024 , reflects a point estimate of the indirect effect of abusive 
supervision on CWBI via PA and has a bootstrap standard error of 0.015 . The bootstrap confidence interval about the indirect effect has a lower limit of -0.058 and an upper limit of -0.001 , which excludes zero. The indirect effect in Model E3 is significant and thus H1e is supported.

\section{Mediating Effect of Negative Affect}

Tables 10a-10e display a summary of the models estimated to test Hypotheses $2 \mathrm{a}-$ $2 e$, respectively. Hypotheses $2 a-2 e$ reference the mediating effects of NA on each of the five job performance outcomes.

Hypothesis 2a (H2a). As shown on Table 10a, the unstandardized regression coefficient associated with abusive supervision $(\mathrm{B}=0.608)$ is significant in Model A1 and has a bootstrap standard error of 0.075. In addition, the coefficient associated with NA (B $=0.026)$ is not significant in Model A2 and has a bootstrap standard error of 0.051 . The

product of these coefficients, 0.016 , reflects a point estimate of the indirect effect of abusive supervision on task performance via NA and has a bootstrap standard error of 0.032. The bootstrap confidence interval about the indirect effect has a lower limit of 0.041 and an upper limit of 0.085 , which includes zero. The indirect effect in Model A3 is not significant and thus H2a is not supported.

Hypothesis 2b (H2b). As shown on Table 10b, the unstandardized regression coefficient associated with abusive supervision $(B=0.608)$ is significant in Model B1 and has a bootstrap standard error of 0.074 . In addition, the coefficient associated with NA ( $B$ $=0.048)$ is not significant in Model B2 and has a bootstrap standard error of 0.067. The product of these coefficients, 0.029 , is point estimate of the indirect effect of abusive supervision on OCBO via NA and has a bootstrap standard error of 0.042 . The bootstrap 
confidence interval about the indirect effect ranges from -0.049 to 0.116 , which includes zero. The indirect effect in Model B3 is not significant and thus H2b is not supported.

Hypothesis 2c (H2c). As shown on Table 10c, the unstandardized regression coefficient associated with abusive supervision $(B=0.608)$ is significant in Model $C 1$ and has a bootstrap standard error of 0.076 . In addition, the coefficient associated with NA ( $B$ $=0.060)$ is not significant in Model $\mathrm{C} 2$ and has a bootstrap standard error of 0.068 . The product of these coefficients, 0.037 , reflects a point estimate of the indirect effect of abusive supervision on OCBI via NA and has a bootstrap standard error of 0.043 . The bootstrap confidence interval about the indirect effect has a lower limit of -0.045 and an upper limit of 0.124, which includes zero. The indirect effect in Model C3 is not significant and thus $\mathrm{H} 2 \mathrm{c}$ is not supported.

Hypothesis 2d (H2d). As shown on Table 10d, the unstandardized regression coefficient associated with abusive supervision $(B=0.608)$ is significant in Model D1 and has a bootstrap standard error of 0.076 . In addition, the coefficient associated with NA ( $B$ $=0.054)$ is not significant in Model D2 and has a bootstrap standard error of 0.064 . The product of these coefficients, 0.033 , reflects a point estimate of the indirect effect of abusive supervision on CWBO via NA and has a bootstrap standard error of 0.038 . The bootstrap confidence interval about the indirect effect has a lower limit of -0.042 and an upper limit of 0.106, which includes zero. The indirect effect in Model D3 is not significant and thus $\mathrm{H} 2 \mathrm{~d}$ is not supported.

Hypothesis 2e (H2e). As shown on Table 10e, the unstandardized regression coefficient associated with abusive supervision $(B=0.608)$ is significant in Model E1 and has a bootstrap standard error of 0.075 . In addition, the coefficient associated with NA ( $B$ 
$=0.059)$ is not significant in Model E2 and has a bootstrap standard error of 0.056. The product of these coefficients, 0.036 , reflects a point estimate of the indirect effect of abusive supervision on CWBI via NA and has a bootstrap standard error of 0.033 . The bootstrap confidence interval about the indirect effect has a lower limit of -0.034 and an upper limit of 0.097, which includes zero. The indirect effect in Model E3 is not significant and thus H2e is not supported.

\section{Mediating Effect of Leader-Member Exchange}

Tables 11a-11e display a summary of the models estimated to test Hypotheses $3 a-$ 3e, respectively. These hypotheses reference the mediating effects of LMX on each of the five job performance outcomes.

Hypothesis 3a (H3a). As shown on Table 11a, the unstandardized regression coefficient associated with abusive supervision $(B=-0.538)$ is significant in Model A1 and has a bootstrap standard error of 0.073 . In addition, the coefficient associated with LMX $(B=0.041)$ is not significant in Model A2 and has a bootstrap standard error of 0.048 . The product of these coefficients, -0.022 , reflects a point estimate of the indirect effect of abusive supervision on task performance via LMX and has a bootstrap standard error of 0.026. The bootstrap confidence interval about the indirect effect has a lower limit of 0.074 and an upper limit of 0.029 , which includes zero. The indirect effect in Model A3 is not significant and thus H3a is not supported.

Hypothesis 3b (H3b). As shown on Table 11b, the unstandardized regression coefficient associated with abusive supervision $(B=-0.538)$ is significant in Model B1 and has a bootstrap standard error of 0.070 . In addition, the coefficient associated with LMX $(B=0.305)$ is significant in Model B2 and has a bootstrap standard error of 0.065 . The 
product of these coefficients, -0.164 , reflects a point estimate of the indirect effect of abusive supervision on OCBO via LMX and has a bootstrap standard error of 0.041 . The bootstrap confidence interval about the indirect effect has a lower limit of -0.252 and an upper limit of -0.089 , which excludes zero. The indirect effect in Model B3 is significant and thus $\mathrm{H} 3 \mathrm{~b}$ is supported.

Hypothesis 3c (H3c). As shown on Table 11c, the unstandardized regression coefficient associated with abusive supervision $(B=-0.538)$ is significant in Model C1 and has a bootstrap standard error of 0.072. In addition, the coefficient associated with LMX $(B=0.254)$ is significant in Model C2 and has a bootstrap standard error of 0.066 . The product of these coefficients, -0.137 , reflects a point estimate of the indirect effect of abusive supervision on OCBI via LMX and has a bootstrap standard error of 0.041 . The bootstrap confidence interval about the indirect effect has a lower limit of -0.224 and an upper limit of -0.065 , which excludes zero. The indirect effect in Model C3 is significant and thus $\mathrm{H} 3 \mathrm{c}$ is supported.

Hypothesis 3d (H3d). As shown on Table 11d, the unstandardized regression coefficient associated with abusive supervision $(B=-0.538)$ is significant in Model D1 and has a bootstrap standard error of 0.070. In addition, the coefficient associated with LMX $(B=0.199)$ is significant in Model D2 and has a bootstrap standard error of 0.052 . The product of these coefficients, -0.107 , reflects a point estimate of the indirect effect of abusive supervision on CWBO via LMX and has a bootstrap standard error of 0.030 . The bootstrap confidence interval about the indirect effect has a lower limit of -0.170 and an upper limit of -0.050 , which excludes zero. The indirect effect in Model D3 is significant and thus H3d is supported. 
Hypothesis 3e (H3e). As shown on Table 11e, the unstandardized regression coefficient associated with abusive supervision $(B=-0.538)$ is significant in Model E1 and has a bootstrap standard error of 0.072 . In addition, the coefficient associated with LMX $(B=0.185)$ is significant in Model E2 and has a bootstrap standard error of 0.056 . The product of these coefficients, -0.100 , reflects a point estimate of the indirect effect of abusive supervision on CWBI via LMX and has a bootstrap standard error of 0.032 . The bootstrap confidence interval about the indirect effect has a lower limit of -0.166 and an upper limit of -0.039 , which excludes zero. The indirect effect in Model E3 is significant and thus H3e is supported.

\section{Mediating Effect of Interactional Justice}

Tables 12a-12e display a summary of the models estimated to test Hypotheses 4a $-4 \mathrm{e}$, respectively. These hypotheses reference the mediating effects of IJ on each of the five job performance outcomes.

Hypothesis 4a (H4a). As shown on Table 12a, the unstandardized regression coefficient associated with abusive supervision $(B=-0.582)$ is significant in Model A1 and has a bootstrap standard error of 0.083 . In addition, the coefficient associated with IJ ( $B=$ 0.037) is not significant in Model A2 and has a bootstrap standard error of 0.059. The product of these coefficients, -0.022 , reflects a point estimate of the indirect effect of abusive supervision on task performance via IJ and has a bootstrap standard error of 0.034 . The bootstrap confidence interval about the indirect effect has a lower limit of -0.090 and an upper limit of 0.045, which includes zero. The indirect effect in Model A3 is not significant and thus H4a is not supported. 
Hypothesis $4 \mathbf{b}$ (H4b). As shown on Table 12b, the unstandardized regression coefficient associated with abusive supervision $(B=-0.582)$ is significant in Model B1 and has a bootstrap standard error of 0.083 . In addition, the coefficient associated with IJ ( $B=$ 0.314) is significant in Model B2 and has a bootstrap standard error of 0.077 . The product of these coefficients, -0.183 , reflects a point estimate of the indirect effect of abusive supervision on OCBO via IJ and has a bootstrap standard error of 0.050 . The bootstrap confidence interval about the indirect effect has a lower limit of -0.288 and an upper limit of -0.092 , which excludes zero. The indirect effect in Model B3 is significant and thus $\mathrm{H} 4 \mathrm{~b}$ is supported.

Hypothesis $4 \mathbf{c}(\mathbf{H 4 c})$. As shown on Table 12c, the unstandardized regression coefficient associated with abusive supervision $(B=-0.582)$ is significant in Model $C 1$ and has a bootstrap standard error of 0.084 . In addition, the coefficient associated with $\mathrm{IJ}$ ( $B=$ 0.195) is significant in Model C2 and has a bootstrap standard error of 0.076 . The product of these coefficients, -0.113 , reflects a point estimate of the indirect effect of abusive supervision on OCBI via IJ and has a bootstrap standard error of 0.046 . The bootstrap confidence interval about the indirect effect has a lower limit of -0.206 and an upper limit of -0.030 , which excludes zero. The indirect effect in Model $\mathrm{C} 3$ is significant and thus $\mathrm{H} 4 \mathrm{c}$ is supported.

Hypothesis 4d (H4d). As shown on Table 12d, the unstandardized regression coefficient associated with abusive supervision $(B=-0.582)$ is significant in Model D1 and has a bootstrap standard error of 0.081 . In addition, the coefficient associated with IJ ( $B=$ 0.242) is significant in Model D2 and has a bootstrap standard error of 0.046 . The product of these coefficients, -0.141 , is an estimate of the indirect effect of abusive supervision on 
CWBO via IJ and has a bootstrap standard error of 0.032 . The bootstrap confidence interval about the indirect effect ranges from- 0.205 to -0.080 , which excludes zero. The indirect effect in Model D3 is significant and thus H4d is supported.

Hypothesis 4e (H4e). As shown on Table 12e, the unstandardized regression coefficient associated with abusive supervision $(B=-0.582)$ is significant in Model E1 and has a bootstrap standard error of 0.083 . In addition, the coefficient associated with IJ ( $B=$ 0.201 ) is significant in Model E2 and has a bootstrap standard error of 0.051 . The product of these coefficients, -0.117 , reflects a point estimate of the indirect effect of abusive supervision on CWBI via IJ and has a bootstrap standard error of 0.034 . The bootstrap confidence interval about the indirect effect has a lower limit of -0.188 and an upper limit of -0.054 , which excludes zero. The indirect effect in Model E3 is significant and thus H4e is supported.

\section{Mediating Effect of Perceived Organizational Support}

Tables 13a-13e display a summary of the models estimated to test Hypotheses 5a $-5 \mathrm{e}$, respectively. These hypotheses reference the mediating effects of POS on each of the five job performance outcomes.

Hypothesis 5a (H5a). As shown on Table 13a, the unstandardized regression coefficient associated with abusive supervision $(B=-0.404)$ is significant in Model A1 and has a bootstrap standard error of 0.068 . In addition, the coefficient associated with POS ( $B$ $=-0.020)$ is not significant in Model A2 and has a bootstrap standard error of 0.043 . The

product of these coefficients, 0.008 , reflects a point estimate of the indirect effect of abusive supervision on task performance via POS and has a bootstrap standard error of 0.018. The bootstrap confidence interval about the indirect effect has a lower limit of - 
0.026 and an upper limit of 0.047 , which includes zero. The indirect effect in Model A3 is not significant and thus H5a is not supported.

Hypothesis 5b (H5b). As shown on Table 13b, the unstandardized regression coefficient associated with abusive supervision $(B=-0.404)$ is significant in Model B1 and has a bootstrap standard error of 0.068 . In addition, the coefficient associated with POS $(B=0.191)$ is significant in Model B2 and has a bootstrap standard error of 0.070 . The product of these coefficients, -0.077 , reflects a point estimate of the indirect effect of abusive supervision on OCBO via POS and has a bootstrap standard error of 0.030 . The bootstrap confidence interval about the indirect effect has a lower limit of -0.140 and an upper limit of -0.023 , which excludes zero. The indirect effect in Model B3 is significant and thus $\mathrm{H} 5 \mathrm{~b}$ is supported.

Hypothesis 5c (H5c). As shown on Table 13c, the unstandardized regression coefficient associated with abusive supervision $(B=-0.404)$ is significant in Model $C 1$ and has a bootstrap standard error of 0.069. In addition, the coefficient associated with POS ( $B$ $=0.176$ ) is significant in Model C2 and has a bootstrap standard error of 0.070 . The product of these coefficients, -0.071 , reflects a point estimate of the indirect effect of abusive supervision on OCBI via POS and has a bootstrap standard error of 0.030 . The bootstrap confidence interval about the indirect effect has a lower limit of -0.135 and an upper limit of -0.017 , which excludes zero. The indirect effect in Model $\mathrm{C} 3$ is significant and thus $\mathrm{H} 5 \mathrm{c}$ is supported.

Hypothesis 5d (H5d). As shown on Table 13d, the unstandardized regression coefficient associated with abusive supervision $(B=-0.404)$ is significant in Model D1 and has a bootstrap standard error of 0.068 . In addition, the coefficient associated with POS ( $B$ 
$=0.076$ ) is not significant in Model D2 and has a bootstrap standard error of 0.069. The product of these coefficients, -0.031 , reflects a point estimate of the indirect effect of abusive supervision on CWBO via POS and has a bootstrap standard error of 0.030 . The bootstrap confidence interval about the indirect effect has a lower limit of -0.090 and an upper limit of 0.028, which includes zero. The indirect effect in Model D3 is not significant and thus $\mathrm{H} 5 \mathrm{~d}$ is not supported.

Hypothesis 5e (H5e). As shown on Table 13e, the unstandardized regression coefficient associated with abusive supervision $(B=-0.404)$ is significant in Model E1 and has a bootstrap standard error of 0.068. In addition, the coefficient associated with POS ( $B$ $=0.048)$ is not significant in Model E2 and has a bootstrap standard error of 0.069. The product of these coefficients, -0.019 , reflects a point estimate of the indirect effect of abusive supervision on CWBI via POS and has a bootstrap standard error of 0.029 . The bootstrap confidence interval about the indirect effect has a lower limit of -0.074 and an upper limit of 0.038, which includes zero. The indirect effect in Model E3 is not significant and thus H5e is not supported.

\section{Mediating Effect of Affective Organizational Commitment}

Tables $14 a-14$ e display a summary of the models estimated to test Hypotheses $6 \mathrm{a}$ $-6 e$, respectively. These hypotheses reference the mediating effects of AOC on each of the five job performance outcomes.

Hypothesis 6a (H6a). As shown on Table 14a, the unstandardized regression coefficient associated with abusive supervision $(B=-0.274)$ is significant in Model A1 and has a bootstrap standard error of 0.059. In addition, the coefficient associated with AOC $(B=-0.069)$ is not significant in Model A2 and has a bootstrap standard error of 
0.037. The product of these coefficients, 0.019 , reflects a point estimate of the indirect effect of abusive supervision on task performance via AOC and has a bootstrap standard error of 0.012 . The bootstrap confidence interval about the indirect effect has a lower limit of -0.002 and an upper limit of 0.045 , which includes zero. The indirect effect in Model A3 is not significant and thus H6a is not supported.

Hypothesis 6b (H6b). As shown on Table 14b, the unstandardized regression coefficient associated with abusive supervision $(B=-0.274)$ is significant in Model B1 and has a bootstrap standard error of 0.058 . In addition, the coefficient associated with AOC $(B=0.186)$ is significant in Model B2 and has a bootstrap standard error of 0.061 . The product of these coefficients, -0.051 , reflects a point estimate of the indirect effect of abusive supervision on OCBO via AOC and has a bootstrap standard error of 0.021. The bootstrap confidence interval about the indirect effect has a lower limit of -0.099 and an upper limit of -0.016 , which excludes zero. The indirect effect in Model B3 is significant and thus H6b is supported.

Hypothesis 6c (H6c). As shown on Table 14c, the unstandardized regression coefficient associated with abusive supervision $(B=-0.274)$ is significant in Model $C 1$ and has a bootstrap standard error of 0.059 . In addition, the coefficient associated with AOC $(B=0.148)$ is significant in Model C2 and has a bootstrap standard error of 0.061 . The product of these coefficients, -0.040 , reflects a point estimate of the indirect effect of abusive supervision on OCBI via AOC and has a bootstrap standard error of 0.020 . The bootstrap confidence interval about the indirect effect has a lower limit of -0.085 and an upper limit of -0.008 , which excludes zero. The indirect effect in Model C3 is significant and thus H6c is supported. 
Hypothesis 6d (H6d). As shown on Table 14d, the unstandardized regression coefficient associated with abusive supervision $(B=-0.274)$ is significant in Model D1 and has a bootstrap standard error of 0.058 . In addition, the coefficient associated with AOC $(B=0.072)$ is not significant in Model D2 and has a bootstrap standard error of 0.049 . The product of these coefficients, -0.020 , reflects a point estimate of the indirect effect of abusive supervision on CWBO via AOC and has a bootstrap standard error of 0.015 . The bootstrap confidence interval about the indirect effect has a lower limit of -0.052 and an upper limit of 0.009, which includes zero. The indirect effect in Model D3 is not significant and thus H6d is not supported.

Hypothesis 6e (H6e). As shown on Table 14e, the unstandardized regression coefficient associated with abusive supervision $(B=-0.274)$ is significant in Model E1 and has a bootstrap standard error of 0.059 . In addition, the coefficient associated with AOC $(B=0.075)$ is not significant in Model E2 and has a bootstrap standard error of 0.048. The product of these coefficients, -0.021 , reflects a point estimate of the indirect effect of abusive supervision on CWBI via AOC and has a bootstrap standard error of 0.015. The bootstrap confidence interval about the indirect effect has a lower limit of 0.052 and an upper limit of 0.007, which includes zero. The indirect effect in Model E3 is not significant and thus H6e is not supported.

\section{Pairwise Contrasts Between Specific Indirect Effects}

Testing $\mathrm{H} 7 \mathrm{a}-\mathrm{H} 18 \mathrm{e}$ requires estimating five multiple mediator models-one for each job performance outcome of interest—whereby the six mediators operate in "parallel" and the significance of the difference between two specific indirect effects can be formally tested (Hayes, 2017). The specific indirect effects, bootstrap standard errors, and 
confidence intervals about each specific indirect effect, are estimated for each job performance outcome and appear on Tables $15 \mathrm{a}-15 \mathrm{e}$. Recall that in multiple mediator models, the specific indirect effect is interpreted as that mediator's unique ability to account for the effects of abusive supervision on job performance, controlling for the other mediators in the model.

Hypotheses $7 \mathrm{a}-18 \mathrm{e}$ however, reference pairwise contrast across mechanisms postulated in the study. In other words, the difference between two specific indirect effects is estimated and a confidence interval is constructed about the difference. Contrasts with confidence intervals that exclude zero provide evidence that the mechanisms being contrasted differ significantly in strength (i.e., $\mathrm{a}_{1} \mathrm{~b}_{2}-\mathrm{a}_{2} \mathrm{~b}_{2} \neq 0$ ). Therefore, Hypotheses $8 \mathrm{a}$ to $17 \mathrm{e}$ are confirmed if the bootstrap confidence interval (95\%) excludes zero. As noted previously, it is necessary to use the absolute values of the indirect effects (i.e., $\left|a_{1} b_{2}\right|-$ $\left.\left|a_{2} b_{2}\right|\right)$ when the contrast involves specific indirect effects that differ in sign. Otherwise, one may erroneously conclude that two effects of similar magnitude but of different signs (e.g., -.10 vs .10) differ from each other in strength when they do not.

\section{Hypotheses Contrasting the Mediating Effects of PA and Attitudinal}

Mediators. Table 16 displays the contrasts between specific indirect effects associated with PA and AOC. Each row represents the job performance outcome listed in the first column. The point differences between the absolute value of the specific indirect effects associated with PA and the absolute value of the specific indirect effects associated with AOC (i.e., $\left.\left|a_{\mathrm{PA}} b_{\mathrm{PA}}\right|-\left|a_{\mathrm{AOC}} b_{\mathrm{AOC}}\right|\right)$ appear on the second column. The third column displays the bootstrap (5000 samples) standard error associated with the point estimate of 
the difference. The fourth and fifth columns indicate the lower and upper limits of the bootstrap confidence interval for the difference, respectively.

The first row of Table 16 shows the difference between the magnitude of the specific indirect effect associated with PA and the magnitude of the specific indirect effect associated with AOC, in the relationship between abusive supervision and task performance. As shown, the point estimate of the difference is -0.04 (Boot SE $=0.02$ ). The bootstrap confidence interval about the difference — which has a lower limit of -0.07 and an upper limit of 0.01 -includes zero and indicates that the specific indirect effect associated with PA is not significantly weaker than the specific indirect effect associated with AOC. Results from this pairwise contrast fail to support H7a.

The second row of Table 16 shows the difference between the magnitude of the specific indirect effect associated with PA and the magnitude of the specific indirect effect associated with AOC, in the relationship between abusive supervision and OCBO. As shown, the point estimate of the difference is 0.04 (Boot $\mathrm{SE}=0.03$ ). The bootstrap confidence interval about the difference — which has a lower limit of -0.02 and an upper limit of 0.08 -includes zero and indicates that the specific indirect effect associated with PA is not significantly stronger than the specific indirect effect associated with AOC. Results from this pairwise contrast fail to support $\mathrm{H} 7 \mathrm{~b}$.

The third row of Table 16 shows the difference between the magnitude of the specific indirect effect associated with PA and the magnitude of the specific indirect effect associated with AOC, in the relationship between abusive supervision and OCBI. As shown, the point estimate of the difference is 0.02 (Boot $\mathrm{SE}=0.02$ ). The bootstrap confidence interval about the difference - which has a lower limit of -0.03 and an upper 
limit of 0.06 -includes zero and indicates that the specific indirect effect associated with PA is not significantly stronger than the specific indirect effect associated with AOC. Results from this pairwise contrast fail to support $\mathrm{H} 7 \mathrm{c}$.

The fourth row of Table 16 shows the difference between the magnitude of the specific indirect effect associated with PA and the magnitude of the specific indirect effect associated with AOC, in the relationship between abusive supervision and CWBO. As shown, the point estimate of the difference is 0.01 (Boot $\mathrm{SE}=0.02$ ). The bootstrap confidence interval about the difference - which has a lower limit of -0.02 and an upper limit of 0.04 -includes zero and indicates that the specific indirect effect associated with PA is not significantly stronger than the specific indirect effect associated with AOC. Results from this pairwise contrast fail to support $\mathrm{H} 7 \mathrm{~d}$.

The fifth row of Table 16 shows the difference between the magnitude of the specific indirect effect associated with PA and the magnitude of the specific indirect effect associated with AOC, in the relationship between abusive supervision and CWBI. As shown, the point estimate of the difference is 0.03 (Boot $\mathrm{SE}=0.02$ ). The bootstrap confidence interval about the difference - which has a lower limit of -0.02 and an upper limit of 0.07 -includes zero and indicates that the specific indirect effect associated with PA is not significantly stronger than the specific indirect effect associated with AOC. Results from this pairwise contrast fail to support H7e.

The first row of Table 17 shows the difference between the magnitude of the specific indirect effect associated with PA and the magnitude of the specific indirect effect associated with POS, in the relationship between abusive supervision and task performance. As shown, the point estimate of the difference is less than 0.01 (Boot $\mathrm{SE}=$ 
0.02). The bootstrap confidence interval about the difference - which has a lower limit of -0.05 and an upper limit of 0.02 -includes zero and indicates that the specific indirect effect associated with PA is not significantly stronger than the specific indirect effect associated with POS. Results from this pairwise contrast fail to support H8a.

The second row of Table 17 shows the difference between the magnitude of the specific indirect effect associated with PA and the magnitude of the specific indirect effect associated with POS, in the relationship between abusive supervision and OCBO. As shown, the point estimate of the difference is 0.01 (Boot $\mathrm{SE}=0.03$ ). The bootstrap confidence interval about the difference - which has a lower limit of -0.07 and an upper limit of 0.07 -includes zero and indicates that the specific indirect effect associated with PA is not significantly stronger than the specific indirect effect associated with POS. Results from this pairwise contrast fail to support H8b.

The third row of Table 17 shows the difference between the magnitude of the specific indirect effect associated with PA and the magnitude of the specific indirect effect associated with POS, in the relationship between abusive supervision and OCBI. As shown, the point estimate of the difference is 0.01 (Boot $\mathrm{SE}=0.03$ ). The bootstrap confidence interval about the difference - which has a lower limit of - 0.08 and an upper limit of $0.06-$ includes zero and indicates that the specific indirect effect associated with PA is not significantly stronger than the specific indirect effect associated with POS. Results from this pairwise contrast fail to support H8c.

The fourth row of Table 17 shows the difference between the magnitude of the specific indirect effect associated with PA and the magnitude of the specific indirect effect associated with POS, in the relationship between abusive supervision and CWBO. As 
shown, the point estimate of the difference is -0.04 (Boot $\mathrm{SE}=0.04)$. The bootstrap confidence interval about the difference - which has a lower limit of - 0.12 and an upper limit of 0.03 -includes zero and indicates that the specific indirect effect associated with PA is not significantly weaker than the specific indirect effect associated with POS. Results from this pairwise contrast fail to support $\mathrm{H} 8 \mathrm{~d}$.

The fifth row of Table 17 shows the difference between the magnitude of the specific indirect effect associated with PA and the magnitude of the specific indirect effect associated with POS, in the relationship between abusive supervision and CWBI. As shown, the point estimate of the difference is -0.05 (Boot $\mathrm{SE}=0.04)$. The bootstrap confidence interval about the difference - which has a lower limit of - 0.14 and an upper limit of 0.03 -includes zero and indicates that the specific indirect effect associated with PA is not significantly weaker than the specific indirect effect associated with POS. Results from this pairwise contrast fail to support H8e.

The first row of Table 18 shows the difference between the magnitude of the specific indirect effect associated with PA and the magnitude of the specific indirect effect associated with LMX, in the relationship between abusive supervision and task performance. As shown, the point estimate of the difference is -0.04 (Boot $\mathrm{SE}=0.04)$. The bootstrap confidence interval about the difference - which has a lower limit of -0.14 and an upper limit of 0.01 -includes zero and indicates that the specific indirect effect associated with PA is not significantly weaker than the specific indirect effect associated with LMX. Results fail to support H9a.

The second row of Table 18 shows the difference between the magnitude of the specific indirect effect associated with PA and the magnitude of the specific indirect effect 
associated with LMX, in the relationship between abusive supervision and OCBO. As shown, the point estimate of the difference is -0.01 (Boot $\mathrm{SE}=0.05)$. The bootstrap confidence interval about the difference - which has a lower limit of -0.11 and an upper limit of 0.07 -includes zero and indicates that the specific indirect effect associated with PA is not significantly weaker than the specific indirect effect associated with LMX. Results fail to support $\mathrm{H} 9 \mathrm{~b}$.

The third row of Table 18 shows the difference between the magnitude of the specific indirect effect associated with PA and the magnitude of the specific indirect effect associated with LMX, in the relationship between abusive supervision and OCBI. As shown, the point estimate of the difference is -0.07 (Boot $\mathrm{SE}=0.06$ ). The bootstrap confidence interval about the difference - which has a lower limit of -0.19 and an upper limit of 0.03 - includes zero and indicates that the specific indirect effect associated with PA is not significantly weaker than the specific indirect effect associated with LMX. Results fail to support H9c.

The fourth row of Table 18 shows the difference between the magnitude of the specific indirect effect associated with PA and the magnitude of the specific indirect effect associated with LMX, in the relationship between abusive supervision and CWBO. As shown, the point estimate of the difference is -0.01 (Boot $\mathrm{SE}=0.04$ ). The bootstrap confidence interval about the difference - which has a lower limit of -0.11 and an upper limit of 0.04 -includes zero and indicates that the specific indirect effect associated with PA is not significantly weaker than the specific indirect effect associated with LMX. Results fail to support H9d. 
The fifth row of Table 18 shows the difference between the magnitude of the specific indirect effect associated with PA and the magnitude of the specific indirect effect associated with LMX, in the relationship between abusive supervision and CWBI. As shown, the point estimate of the difference is -0.01 (Boot $\mathrm{SE}=0.04)$. The bootstrap confidence interval about the difference- which has a lower limit of -0.12 and an upper limit of 0.05 -includes zero and indicates that the specific indirect effect associated with PA is not significantly weaker than the specific indirect effect associated with LMX. Results fail to support H9e.

The first row of Table 19 shows the difference between the magnitude of the specific indirect effect associated with PA and the magnitude of the specific indirect effect associated with IJ, in the relationship between abusive supervision and task performance. As shown, the point estimate of the difference is -0.02 (Boot $\mathrm{SE}=0.04)$. The bootstrap confidence interval about the difference- which has a lower limit of -0.13 and an upper limit of 0.01 -includes zero and indicates that the specific indirect effect associated with PA is not significantly weaker than the specific indirect effect associated with IJ. Results fail to support H10a.

The second row of Table 19 shows the difference between the magnitude of the specific indirect effect associated with PA and the magnitude of the specific indirect effect associated with IJ, in the relationship between abusive supervision and OCBO. As shown, the point estimate of the difference is -0.04 (Boot $\mathrm{SE}=0.06$ ). The bootstrap confidence interval about the difference - which has a lower limit of - 0.18 and an upper limit of $0.06-$ includes zero and indicates that the specific indirect effect associated with PA is not 
significantly weaker than the specific indirect effect associated with IJ. Results fail to support H10b.

The third row of Table 19 shows the difference between the magnitude of the specific indirect effect associated with PA and the magnitude of the specific indirect effect associated with IJ, in the relationship between abusive supervision and OCBI. As shown, the point estimate of the difference is $-0.01(\mathrm{Boot} \mathrm{SE}=0.05)$. The bootstrap confidence interval about the difference - which has a lower limit of - 0.14 and an upper limit of $0.04-$ includes zero and indicates that the specific indirect effect associated with PA is not significantly weaker than the specific indirect effect associated with IJ. Results fail to support H10c.

The fourth row of Table 19 shows the difference between the magnitude of the specific indirect effect associated with PA and the magnitude of the specific indirect effect associated with IJ, in the relationship between abusive supervision and CWBO. As shown, the point estimate of the difference is $-0.12($ Boot $\mathrm{SE}=0.05)$. The bootstrap confidence interval about the difference - which has a lower limit of -0.22 and an upper limit of 0.01-excludes zero and indicates that the specific indirect effect associated with PA is significantly weaker than the specific indirect effect associated with IJ. Results fail to support H10d.

The fifth row of Table 19 shows the difference between the magnitude of the specific indirect effect associated with PA and the magnitude of the specific indirect effect associated with IJ, in the relationship between abusive supervision and CWBI. As shown, the point estimate of the difference is -0.06 (Boot $\mathrm{SE}=0.05)$. The bootstrap confidence interval about the difference - which has a lower limit of - 0.16 and an upper limit of $0.03-$ 
includes zero and indicates that the specific indirect effect associated with PA is not significantly weaker than the specific indirect effect associated with IJ. Results fail to support H10e.

\section{Hypotheses Contrasting the Mediating Effect of NA to Attitudinal Mediators.}

The first row of Table 20 shows the difference between the magnitude of the specific indirect effect associated with NA and the magnitude of the specific indirect effect associated with AOC, in the relationship between abusive supervision and task performance. As shown, the point estimate of the difference is -0.03 (Boot $\mathrm{SE}=0.03$ ). The bootstrap confidence interval about the difference-which has a lower limit of -0.06 and an upper limit of 0.06 - includes zero and indicates that the specific indirect effect associated with NA is not significantly weaker than the specific indirect effect associated with AOC. Results fail to support H11a.

The second row of Table 20 shows the difference between the magnitude of the specific indirect effect associated with NA and the magnitude of the specific indirect effect associated with AOC, in the relationship between abusive supervision and OCBO. As shown, the point estimate of the difference is 0.13 (Boot $\mathrm{SE}=0.05$ ). The bootstrap confidence interval about the difference-which has a lower limit of 0.01 and an upper limit of 0.22 - excludes zero and indicates that the specific indirect effect associated with NA is significantly stronger than the specific indirect effect associated with AOC. Results support H11b.

The third row of Table 20 shows the difference between the magnitude of the specific indirect effect associated with NA and the magnitude of the specific indirect effect associated with AOC, in the relationship between abusive supervision and OCBI. As 
shown, the point estimate of the difference is 0.12 (Boot $\mathrm{SE}=0.05$ ). The bootstrap confidence interval about the difference - which has a lower limit of 0.00 and an upper limit of 0.21 —excludes zero and indicates that the specific indirect effect associated with NA is significantly stronger than the specific indirect effect associated with AOC. Results support H11c.

The fourth row of Table 20 shows the difference between the magnitude of the specific indirect effect associated with NA and the magnitude of the specific indirect effect associated with $\mathrm{AOC}$, in the relationship between abusive supervision and CWBO. As shown, the point estimate of the difference is 0.06 (Boot $\mathrm{SE}=0.04)$. The bootstrap confidence interval about the difference - which has a lower limit of -0.02 and an upper limit of 0.13 -includes zero and indicates that the specific indirect effect associated with NA is not significantly stronger than the specific indirect effect associated with AOC. Results support H11d.

The fifth row of Table 20 shows the difference between the magnitude of the specific indirect effect associated with NA and the magnitude of the specific indirect effect associated with AOC, in the relationship between abusive supervision and CWBI. As shown, the point estimate of the difference is 0.08 (Boot $\mathrm{SE}=0.04$ ). The bootstrap confidence interval about the difference- which has a lower limit of -0.01 and an upper limit of 0.14 -includes zero and indicates that the specific indirect effect associated with NA is not significantly stronger than the specific indirect effect associated with AOC. Results fail to support H11e.

The first row of Table 21 shows the difference between the magnitude of the specific indirect effect associated with NA and the magnitude of the specific indirect effect 
associated with POS, in the relationship between abusive supervision and task performance. As shown, the point estimate of the difference is less than 0.01 (Boot SE $=$ 0.03). The bootstrap confidence interval about the difference-which has a lower limit of -0.04 and an upper limit of 0.08 - includes zero and indicates that the specific indirect effect associated with NA is not significantly stronger than the specific indirect effect associated with POS. Results fail to support H12a.

The second row of Table 21 shows the difference between the magnitude of the specific indirect effect associated with NA and the magnitude of the specific indirect effect associated with POS, in the relationship between abusive supervision and OCBO. As shown, the point estimate of the difference is 0.09 (Boot $\mathrm{SE}=0.06$ ). The bootstrap confidence interval about the difference - which has a lower limit of - -0.02 and an upper limit of 0.21 -includes zero and indicates that the specific indirect effect associated with NA is not significantly stronger than the specific indirect effect associated with POS. Results fail to support H12b.

The third row of Table 21 shows the difference between the magnitude of the specific indirect effect associated with NA and the magnitude of the specific indirect effect associated with POS, in the relationship between abusive supervision and OCBI. As shown, the point estimate of the difference is 0.10 (Boot $\mathrm{SE}=0.06)$. The bootstrap confidence interval about the difference- - which has a lower limit of - 0.02 and an upper limit of $0.20-$ includes zero and indicates that the specific indirect effect associated with NA is not significantly stronger than the specific indirect effect associated with POS. Results fail to support H12c. 
The fourth row of Table 21 shows the difference between the magnitude of the specific indirect effect associated with NA and the magnitude of the specific indirect effect associated with POS, in the relationship between abusive supervision and CWBO. As shown, the point estimate of the difference is 0.01 (Boot $\mathrm{SE}=0.06$ ). The bootstrap confidence interval about the difference — which has a lower limit of -0.12 and an upper limit of 0.12 -includes zero and indicates that the specific indirect effect associated with NA is not significantly stronger than the specific indirect effect associated with POS. Results fail to support H12d.

The fifth row of Table 21 shows the difference between the magnitude of the specific indirect effect associated with NA and the magnitude of the specific indirect effect associated with POS, in the relationship between abusive supervision and CWBI. As shown, the point estimate of the difference is less than 0.01 (Boot SE $=0.07)$. The bootstrap confidence interval about the difference - which has a lower limit of -0.14 and an upper limit of 0.12 -includes zero and indicates that the specific indirect effect associated with NA is not significantly stronger than the specific indirect effect associated with POS. Results fail to support H12e.

The first row of Table 22 shows the difference between the magnitude of the specific indirect effect associated with NA and the magnitude of the specific indirect effect associated with LMX, in the relationship between abusive supervision and task performance. As shown, the point estimate of the difference is -0.04 (Boot SE $=0.05$ ). The bootstrap confidence interval about the difference - which has a lower limit of -0.12 and an upper limit of 0.06 -includes zero and indicates that the specific indirect effect 
associated with NA is not significantly weaker than the specific indirect effect associated with LMX. Results fail to support H13a.

The second row of Table 22 shows the difference between the magnitude of the specific indirect effect associated with NA and the magnitude of the specific indirect effect associated with LMX, in the relationship between abusive supervision and OCBO. As shown, the point estimate of the difference is 0.08 (Boot $\mathrm{SE}=0.07$ ). The bootstrap confidence interval about the difference- which has a lower limit of -0.07 and an upper limit of 0.21 -includes zero and indicates that the specific indirect effect associated with NA is not significantly stronger than the specific indirect effect associated with LMX. Results fail to support H13b.

The third row of Table 22 shows the difference between the magnitude of the specific indirect effect associated with NA and the magnitude of the specific indirect effect associated with LMX, in the relationship between abusive supervision and OCBI. As shown, the point estimate of the difference is 0.02 (Boot $\mathrm{SE}=0.08)$. The bootstrap confidence interval about the difference- - which has a lower limit of -0.14 and an upper limit of 0.17 -includes zero and indicates that the specific indirect effect associated with NA is not significantly stronger than the specific indirect effect associated with LMX. Results fail to support H13c.

The fourth row of Table 22 shows the difference between the magnitude of the specific indirect effect associated with NA and the magnitude of the specific indirect effect associated with LMX, in the relationship between abusive supervision and CWBO. As shown, the point estimate of the difference is 0.04 (Boot $\mathrm{SE}=0.05$ ). The bootstrap confidence interval about the difference- which has a lower limit of -0.09 and an upper 
limit of 0.12 -includes zero and indicates that the specific indirect effect associated with NA is not significantly stronger than the specific indirect effect associated with LMX. Results fail to support H13d.

The fifth row of Table 22 shows the difference between the magnitude of the specific indirect effect associated with NA and the magnitude of the specific indirect effect associated with LMX, in the relationship between abusive supervision and CWBI. As shown, the point estimate of the difference is 0.04 (Boot $\mathrm{SE}=0.06$ ). The bootstrap confidence interval about the difference - which has a lower limit of -0.10 and an upper limit of 0.13 -includes zero and indicates that the specific indirect effect associated with NA is not significantly stronger than the specific indirect effect associated with LMX. Results fail to support H13e.

The first row of Table 23 shows the difference between the magnitude of the specific indirect effect associated with NA and the magnitude of the specific indirect effect associated with IJ, in the relationship between abusive supervision and task performance. As shown, the point estimate of the difference is $-0.02($ Boot $\mathrm{SE}=0.04)$. The bootstrap confidence interval about the difference - which has a lower limit of - 0.11 and an upper limit of 0.06-includes zero and indicates that the specific indirect effect associated with NA is not significantly weaker than the specific indirect effect associated with IJ. Results fail to support H14a.

The second row of Table 23 shows the difference between the magnitude of the specific indirect effect associated with NA and the magnitude of the specific indirect effect associated with IJ, in the relationship between abusive supervision and OCBO. As shown, the point estimate of the difference is 0.05 (Boot $\mathrm{SE}=0.08$ ). The bootstrap confidence 
interval about the difference - which has a lower limit of - 0.11 and an upper limit of $0.19-$ includes zero and indicates that the specific indirect effect associated with NA is not significantly stronger than the specific indirect effect associated with IJ. Results fail to support H14b.

The third row of Table 23 shows the difference between the magnitude of the specific indirect effect associated with NA and the magnitude of the specific indirect effect associated with IJ, in the relationship between abusive supervision and OCBI. As shown, the point estimate of the difference is 0.08 (Boot $\mathrm{SE}=0.07$ ). The bootstrap confidence interval about the difference — which has a lower limit of -0.09 and an upper limit of $0.19-$ includes zero and indicates that the specific indirect effect associated with NA is not significantly stronger than the specific indirect effect associated with IJ. Results fail to support H14c.

The fourth row of Table 23 shows the difference between the magnitude of the specific indirect effect associated with NA and the magnitude of the specific indirect effect associated with IJ, in the relationship between abusive supervision and CWBO. As shown, the point estimate of the difference is -0.07 (Boot $\mathrm{SE}=0.07$ ). The bootstrap confidence interval about the difference - which has a lower limit of -0.20 and an upper limit of $0.07-$ includes zero and indicates that the specific indirect effect associated with NA is not significantly weaker than the specific indirect effect associated with IJ. Results fail to support H14d.

The fifth row of Table 23 shows the difference between the magnitude of the specific indirect effect associated with NA and the magnitude of the specific indirect effect associated with IJ, in the relationship between abusive supervision and CWBI. As shown, 
the point estimate of the difference is -0.01 (Boot $\mathrm{SE}=0.06$ ). The bootstrap confidence interval about the difference — which has a lower limit of - 0.14 and an upper limit of $0.11-$ includes zero and indicates that the specific indirect effect associated with NA is not significantly weaker than the specific indirect effect associated with IJ. Results fail to support $\mathrm{H} 14 \mathrm{e}$.

\section{Hypotheses contrasting the mediating effect of AOC to supervisor-referenced}

mediators. The first row of Table 24 shows the difference between the magnitude of the specific indirect effect associated with AOC and the magnitude of the specific indirect effect associated with LMX, in the relationship between abusive supervision and task performance. As shown, the point estimate of the difference is -0.01 (Boot SE =0.04). The bootstrap confidence interval about the difference - which has a lower limit of -0.11 and an upper limit of 0.05 -includes zero and indicates that the specific indirect effect associated with AOC is not significantly weaker than the specific indirect effect associated with LMX. Results fail to support H15a.

The second row of Table 24 shows the difference between the magnitude of the specific indirect effect associated with AOC and the magnitude of the specific indirect effect associated with LMX, in the relationship between abusive supervision and OCBO. As shown, the point estimate of the difference is 0.05 (Boot $\mathrm{SE}=0.08$ ). The bootstrap confidence interval about the difference - which has a lower limit of -0.11 and an upper limit of 0.19 - includes zero and indicates that the specific indirect effect associated with AOC is not significantly stronger than the specific indirect effect associated with LMX. Results fail to support H15b. 
The third row of Table 24 shows the difference between the magnitude of the specific indirect effect associated with $\mathrm{AOC}$ and the magnitude of the specific indirect effect associated with LMX, in the relationship between abusive supervision and OCBI. As shown, the point estimate of the difference is $-0.09(\mathrm{Boot} \mathrm{SE}=0.06)$. The bootstrap confidence interval about the difference - which has a lower limit of -0.20 and an upper limit of 0.01 -includes zero and indicates that the specific indirect effect associated with AOC is not significantly weaker than the specific indirect effect associated with LMX. Results fail to support $\mathrm{H} 15 \mathrm{c}$.

The fourth row of Table 24 shows the difference between the magnitude of the specific indirect effect associated with $\mathrm{AOC}$ and the magnitude of the specific indirect effect associated with LMX, in the relationship between abusive supervision and CWBO. As shown, the point estimate of the difference is -0.02 (Boot $\mathrm{SE}=0.04)$. The bootstrap confidence interval about the difference - which has a lower limit of -0.11 and an upper limit of 0.03 -includes zero and indicates that the specific indirect effect associated with AOC is not significantly weaker than the specific indirect effect associated with LMX. Results fail to support H15d.

The fifth row of Table 24 shows the difference between the magnitude of the specific indirect effect associated with $\mathrm{AOC}$ and the magnitude of the specific indirect effect associated with LMX, in the relationship between abusive supervision and CWBI. As shown, the point estimate of the difference is -0.04 (Boot SE $=0.04)$. The bootstrap confidence interval about the difference- which has a lower limit of - 0.14 and an upper limit of 0.02 - includes zero and indicates that the specific indirect effect associated with 
AOC is not significantly weaker than the specific indirect effect associated with LMX. Results fail to support H15e.

The first row of Table 25 shows the difference between the magnitude of the specific indirect effect associated with AOC and the magnitude of the specific indirect effect associated with $\mathrm{IJ}$, in the relationship between abusive supervision and task performance. As shown, the point estimate of the difference is $0.01($ Boot SE $=0.04)$. The bootstrap confidence interval about the difference - which has a lower limit of -0.10 and an upper limit of 0.05 -includes zero and indicates that the specific indirect effect associated with AOC is not significantly stronger than the specific indirect effect associated with IJ. Results fail to support H16a.

The second row of Table 25 shows the difference between the magnitude of the specific indirect effect associated with AOC and the magnitude of the specific indirect effect associated with IJ, in the relationship between abusive supervision and OCBO. As shown, the point estimate of the difference is -0.08 (Boot $\mathrm{SE}=0.06$ ). The bootstrap confidence interval about the difference - which has a lower limit of -0.20 and an upper limit of 0.02 -includes zero and indicates that the specific indirect effect associated with AOC is not significantly weaker than the specific indirect effect associated with IJ. Results fail to support H16b.

The third row of Table 25 shows the difference between the magnitude of the specific indirect effect associated with AOC and the magnitude of the specific indirect effect associated with IJ, in the relationship between abusive supervision and OCBI. As shown, the point estimate of the difference is -0.04 (Boot $\mathrm{SE}=0.05)$. The bootstrap confidence interval about the difference- which has a lower limit of - 0.16 and an upper 
limit of 0.03 -includes zero and indicates that the specific indirect effect associated with AOC is not significantly weaker than the specific indirect effect associated with IJ. Results fail to support H16c.

The fourth row of Table 25 shows the difference between the magnitude of the specific indirect effect associated with AOC and the magnitude of the specific indirect

effect associated with IJ, in the relationship between abusive supervision and CWBO. As shown, the point estimate of the difference is -0.13 (Boot $\mathrm{SE}=0.05$ ). The bootstrap confidence interval about the difference — which has a lower limit of -0.23 and an upper limit of -0.02 - excludes zero and indicates that the specific indirect effect associated with AOC is significantly weaker than the specific indirect effect associated with IJ. Results fail to support H16d.

The fifth row of Table 25 shows the difference between the magnitude of the specific indirect effect associated with $\mathrm{AOC}$ and the magnitude of the specific indirect effect associated with IJ, in the relationship between abusive supervision and CWBI. As shown, the point estimate of the difference is -0.09 (Boot $\mathrm{SE}=0.05$ ). The bootstrap confidence interval about the difference - which has a lower limit of -0.18 and an upper limit of 0.00 -includes zero and indicates that the specific indirect effect associated with AOC is not significantly weaker than the specific indirect effect associated with IJ. Results fail to support H16e.

\section{Hypotheses Contrasting the Mediating Effect of POS to Supervisor-} Referenced Mediators. The first row of Table 26 shows the difference between the magnitude of the specific indirect effect associated with POS and the magnitude of the specific indirect effect associated with LMX, in the relationship between abusive 
supervision and task performance. As shown, the point estimate of the difference is -0.04 $($ Boot $\mathrm{SE}=0.04)$. The bootstrap confidence interval about the difference-which has a lower limit of -0.13 and an upper limit of 0.03 - includes zero and indicates that the specific indirect effect associated with POS is not significantly weaker than the specific indirect effect associated with LMX. Results fail to support H17a.

The second row of Table 26 shows the difference between the magnitude of the specific indirect effect associated with POS and the magnitude of the specific indirect effect associated with LMX, in the relationship between abusive supervision and OCBO. As shown, the point estimate of the difference is -0.01 (Boot $\mathrm{SE}=0.05$ ). The bootstrap confidence interval about the difference - which has a lower limit of -0.12 and an upper limit of 0.08 -includes zero and indicates that the specific indirect effect associated with POS is not significantly weaker than the specific indirect effect associated with LMX. Results fail to support $\mathrm{H} 17 \mathrm{~b}$.

The third row of Table 26 shows the difference between the magnitude of the specific indirect effect associated with POS and the magnitude of the specific indirect effect associated with LMX, in the relationship between abusive supervision and OCBI. As shown, the point estimate of the difference is -0.08 (Boot $\mathrm{SE}=0.06$ ). The bootstrap confidence interval about the difference- which has a lower limit of -0.19 and an upper limit of 0.05 -includes zero and indicates that the specific indirect effect associated with POS is not significantly weaker than the specific indirect effect associated with LMX. Results fail to support H17c.

The fourth row of Table 26 shows the difference between the magnitude of the specific indirect effect associated with POS and the magnitude of the specific indirect effect 
associated with LMX, in the relationship between abusive supervision and CWBO. As shown, the point estimate of the difference is 0.03 (Boot $\mathrm{SE}=0.04$ ). The bootstrap confidence interval about the difference- - which has a lower limit of -0.07 and an upper limit of 0.10 -includes zero and indicates that the specific indirect effect associated with POS is not significantly stronger than the specific indirect effect associated with LMX. Results fail to support H17d.

The fifth row of Table 26 shows the difference between the magnitude of the specific indirect effect associated with POS and the magnitude of the specific indirect effect associated with LMX, in the relationship between abusive supervision and CWBI. As shown, the point estimate of the difference is 0.04 (Boot $\mathrm{SE}=0.05$ ). The bootstrap confidence interval about the difference - which has a lower limit of -0.06 and an upper limit of 0.12 -includes zero and indicates that the specific indirect effect associated with POS is not significantly stronger than the specific indirect effect associated with LMX. Results fail to support H17e.

The first row of Table 27 shows the difference between the magnitude of the specific indirect effect associated with POS and the magnitude of the specific indirect effect associated with IJ, in the relationship between abusive supervision and task performance. As shown, the point estimate of the difference is -0.02 (Boot $\mathrm{SE}=0.04$ ). The bootstrap confidence interval about the difference - which has a lower limit of -0.12 and an upper limit of 0.04 -includes zero and indicates that the specific indirect effect associated with POS is not significantly weaker than the specific indirect effect associated with IJ. Results fail to support H18a. 
The second row of Table 27 shows the difference between the magnitude of the specific indirect effect associated with POS and the magnitude of the specific indirect effect associated with IJ, in the relationship between abusive supervision and OCBO. As shown, the point estimate of the difference is -0.04 (Boot $\mathrm{SE}=0.06$ ). The bootstrap confidence interval about the difference — which has a lower limit of -0.17 and an upper limit of $0.05-$ includes zero and indicates that the specific indirect effect associated with POS is not significantly weaker than the specific indirect effect associated with IJ. Results fail to support H18b.

The third row of Table 27 shows the difference between the magnitude of the specific indirect effect associated with POS and the magnitude of the specific indirect effect associated with IJ, in the relationship between abusive supervision and OCBI. As shown, the point estimate of the difference is $-0.02($ Boot $\mathrm{SE}=0.05$ ). The bootstrap confidence interval about the difference - which has a lower limit of - 0.14 and an upper limit of $0.06-$ includes zero and indicates that the specific indirect effect associated with POS is not significantly weaker than the specific indirect effect associated with IJ. Results fail to support H18c.

The fourth row of Table 27 shows the difference between the magnitude of the specific indirect effect associated with POS and the magnitude of the specific indirect effect associated with IJ, in the relationship between abusive supervision and CWBO. As shown, the point estimate of the difference is -0.08 (Boot $\mathrm{SE}=0.05$ ). The bootstrap confidence interval about the difference - which has a lower limit of - 0.19 and an upper limit of $0.02-$ includes zero and indicates that the specific indirect effect associated with POS is not 
significantly weaker than the specific indirect effect associated with IJ. Results fail to support H18d.

The fifth row of Table 27 shows the difference between the magnitude of the specific indirect effect associated with POS and the magnitude of the specific indirect effect associated with IJ, in the relationship between abusive supervision and CWBI. As shown, the point estimate of the difference is -0.01 (Boot $\mathrm{SE}=0.05)$. The bootstrap confidence interval about the difference - which has a lower limit of - 0.12 and an upper limit of $0.09-$ includes zero and indicates that the specific indirect effect associated with POS is not significantly weaker than the specific indirect effect associated with IJ. Results fail to support H18e.

\section{Role of PEC}

The remaining hypotheses reference the role of PEC and are tested using the unmatched sample $(N=2738)$. As noted above, the sample data distribution exhibits strong departures from normality at the univariate and multivariate levels. Therefore, nonparametric correlations (Spearman's rho) are used to test hypothesis 19 and the PROCESS (Hayes, 2018) application is used to bootstrap the standard errors and construct bootstrap confidence intervals for the coefficients in the OLS moderated multiple regression models used to test hypotheses 20 and 21 .

\section{Correlational Analyses}

Hypotheses 19a - 19e are tested via bivariate (zero-order) correlations and confirmed if the correlation is significantly different from zero in the expected direction. As shown on Table 7a, the (Spearman's $\rho$ ) correlation coefficient associated with the relationship between PEC and AS $(\rho=-.35, p<.05)$ is negative and statistically significant. 
Similarly, the correlation coefficients associated with the relationship between employeerated PEC and (1) AOC ( $\rho=.55),(2) \operatorname{POS}(\rho=.68)$, (3) LMX $(\rho=.52)$, and (4) IJ ( $\rho=$ .62 ), are positive and significantly greater than zero (i.e., $p<.05$ ). Results from the nonparametric correlational analyses support hypothesis 19 (a-e).

\section{Confirmatory Factor Analyses}

Hypotheses $20 \mathrm{a}-21 \mathrm{~b}$ refer to the moderating effects of employee's perceived ethical climate (PEC). The 22-item PEC instrument used in the present study was developed by Hsieh and Wang (2016) based on items written by Victor and Cullen (1988). The instrument purports to measure PEC as a "global construct [second-order]" composed of five first order factors — caring, law and code, rules, independence, and instrumental— that represent the distinct EOCs articulated by ECT. It is necessary to evaluate the extent to which this instrument conforms with the expected higher order structure. Higher-order CFAs were conducted in LISREL (v.10; Jöreskog \& Sörbom, 2018) with the maximum likelihood (ML) estimator. Although items are measured on ordinal scales, they are treated as continuous indicator in the present analyses. As noted by Iaccobucci (2007), this is common practice in management research when items have 5 or more response categories.

An initial second-order CFA (five first-order factors plus one second order factor), which included the full set of items, yields a poor fit $(\chi 2[\mathrm{df}=204]=4251.31$, RMSEA $=$ $.09, \mathrm{CFI}=.85, \mathrm{SRMR}=.12$ ). Two modifications are made to bring the select fit indices within an acceptable range. First, items 19 and 21 are dropped from the Instrumental subscale. Second, the residuals of (1) Independence and Caring and (2) Independence and Instrumental, are allowed to correlate (rather than specified as zero). These two modifications yielded a model with an acceptable fit $(\chi 2[\mathrm{df}=163]=2272.56, \mathrm{RMSEA}=$ 
$.07, \mathrm{CFI}=.92, \mathrm{SRMR}=.06)$. Moreover, the average variance extracted (AVE) exceeds Fornell and Larcker's (1984) conservative .50 threshold for all but one first-order factor and the mean AVE across the first-order factors is .53.

Figures 2 and 3 display the standardized solutions for the initial and final secondorder CFAs, respectively. The results confirm expectations regarding the hierarchical structure of PEC. The Cronbach's alpha are $.80, .83, .84, .77$, and .82 , for the (non) instrumental, caring, independence, rules, and law and code climates, respectively. The Cronbach's alpha for the overall (modified) scale is .82 . Further, the original and modified PEC scales have a positive zero-order correlation that approaches unity (Pearson $r=.98$, Spearman $\rho=.98)$.

\section{Moderated Multiple Regression}

Moderated multiple regression (MMR) analyses were conducted to evaluate Hypotheses 20 (a-b) and $21(a-b)$, which stipulate the moderating effect of PEC in the relationship between abusive supervision and each employee attitude of interest (e.g., organization-related, supervisor-related). Across MMR models, the attitude of interest is treated as the outcome variable. The models specified to test $\mathrm{H} 20 \mathrm{a}-\mathrm{H} 21 \mathrm{~b}$ are summarized below and the results are displayed on Tables $28 \mathrm{a}-29 \mathrm{~b}$, respectively.

Two OLS regression models are estimated to test each hypothesis. In the first model (Model 1), the variables corresponding to abusive supervision and PEC variables are entered and a multiple $\mathrm{R}^{2}$ is calculated. The second model (Model 2) includes the same variables as the first model, with the addition of the variable representing the product of abusive supervision and PEC. The significance of the difference between the multiple $\mathrm{R}^{2}$ of the second model and the multiple $\mathrm{R}^{2}$ of the first model (i.e., increase in multiple $\mathrm{R}^{2}$ ) is 
tested using an F-ratio test. A significant F-test suggests the second model explains a significantly greater amount of variance in the dependent variable than the first model. Since the only difference between the models is the addition of the interaction term, a significant test provides evidence that a nontrivial portion of the variance in the dependent variable is explained by the interaction of abusive supervision and PEC.

\section{Hypotheses Related to the Moderating Effects of PEC on Supervisor-related}

Attitudes. As shown under Model 1 on Table 28a, the unstandardized regression coefficient associated with $\mathrm{AS}(B=-0.20, \mathrm{p}<.05 ; 95 \%$ Boot $\mathrm{CI}=[-0.22,-0.17])$ is significant, as is the unstandardized regression coefficient associated with PEC $(B=0.79$, $\mathrm{p}<.05 ; 95 \%$ Boot $\mathrm{CI}=[0.73,0.86])$. When the interaction term is added the model (Model 2 ), the coefficient associated with this term is significant $(B=0.16, \mathrm{p}<.05 ; 95 \%$ Boot CI $=[0.10,0.13])$. Moreover, the addition of the interaction term produces a significant increase in the variance explained by the model (increase in Multiple $\mathrm{R}^{2}=.01, \mathrm{p}<.05$ ). To probe the interaction, simple slopes of the relationship between abusive supervision and LMX were plotted at values of PEC one standard deviation above and one standard deviation below the mean of PEC, which corresponds to high and low values, respectively (see Figure 4). Despite evidence supporting the moderating role of PEC, the pattern of simple effects is contrary to predictions and fails to support H20a.

As shown under Model 1 on Table 28b, the unstandardized regression coefficient associated with AS $(B=-0.11, \mathrm{p}<.05 ; 95 \%$ Boot $\mathrm{CI}=[-0.14,-0.09])$ is significant, as is the unstandardized regression coefficient associated with $\operatorname{PEC~}(B=1.01, \mathrm{p}<.05 ; 95 \%$ Boot $\mathrm{CI}=[0.95,1.08])$. When the interaction term is added the model (Model 2), the coefficient associated with this term is significant $(B=0.34, \mathrm{p}<.05 ; 95 \%$ Boot $\mathrm{CI}=[0.29,0.39])$. 
Moreover, the addition of the interaction term produces a significant increase in the variance explained by the model (increase in Multiple $\mathrm{R}^{2}=.05, \mathrm{p}<.05$ ). To probe the interaction, simple slopes of the relationship between abusive supervision and IJ were plotted at high and low values of PEC (see Figure 5). Despite evidence to support the moderating role of PEC, the pattern of simple effects is contrary to predictions and fails to support H20b.

\section{Hypotheses Related to the Moderating Effects of PEC on Organization-related}

Attitudes. As shown under Model 1 on Table 29a, the unstandardized regression coefficient associated with $\mathrm{AS}(B=-0.10, \mathrm{p}<.05 ; 95 \%$ Boot $\mathrm{CI}=[-0.11,-0.08])$ is significant, as is the unstandardized regression coefficient associated with PEC $(B=1.06$, $\mathrm{p}<.05 ; 95 \%$ Boot $\mathrm{CI}=[1.02,1.11])$. When the interaction term is added the model (Model $2)$, the coefficient associated with this term is not significant $(B=-.00, \mathrm{p}>.05 ; 95 \%$ Boot $\mathrm{CI}=[-0.04,0.04])$. Moreover, the addition of the interaction term does not produce a significant increase in the variance explained by the model (increase in Multiple $\mathrm{R}^{2}=.00$, $\mathrm{p}>.05)$. Results fail to support H21a.

As shown under Model 1 on Table 29b, the unstandardized regression coefficient associated with AS $(B=-0.13, \mathrm{p}<.05 ; 95 \%$ Boot $\mathrm{CI}=[-0.15,-0.11])$ is significant, as is the unstandardized regression coefficient associated with PEC $(B=0.85, \mathrm{p}<.05 ; 95 \%$ Boot $\mathrm{CI}=[0.80,0.90])$. When the interaction term is added the model (Model 2), the coefficient associated with this term is significant $(B=-0.16, \mathrm{p}<.05 ; 95 \%$ Boot $\mathrm{CI}=[-0.20,-0.11])$. Moreover, the addition of the interaction term produces a significant increase in the variance explained by the model (increase in Multiple $\mathrm{R}^{2}=.01, \mathrm{p}<.05$ ). To probe the interaction, simple slopes of the relationship between abusive supervision and AOC were 
plotted at high and low values of PEC (see Figure 6). Despite evidence to support the moderating role of $\mathrm{PEC}$, the pattern of simple effects is contrary to predictions and fails to support H21b.

\section{Structural Equation Modeling}

The larger sample size of the unmatched dataset $(N=2738)$ makes a latent variable approach feasible for hypotheses 20 and 21. Eight-factor measurement models (i.e., CFAs) are estimated using ML The latent factors in each measurement model include (1) the modified PEC measure and its five first order factors, (2) AS, and (3) the outcome of interest (e.g., LMX, IJ, AOC, POS). As shown on Table 30a, Models 1 and 2, corresponding to LMX and IJ, respectively, showed acceptable fit statistics (given the characteristics of this sample).

Models 3 and 4 however, were initially a poor fit to the data and thus required modifications. Model 3 was modified by reducing the number of manifest indicators of POS from eight to six (removed items \#6 and \#7; see Appendix). Similarly, Model 4 showed an acceptable fit once four of the eight indicators of AOC (items \#1 - \#4) were removed. Invariably, the items removed from each factor were those with the lowest loadings. No other modifications were made.

Latent variable scores were then estimated and used to construct the interaction term (Joreskog, Sorbom, \& Wallentin, 2006). Finally, the attitude of interest (outcome variable) is regressed on the latent variable scores of (1) AS, (2) PEC, and (3) the product

of AS and PEC. The estimated regression equations are presented in Table 30b. Results of the latent variable analyses yield identical conclusions to the manifest variable analyses reported above. 


\section{Chapter Summary}

The data cleaning protocol and characteristics of the final datasets are described. Usable data was obtained for 2738 individuals, from which 281 had a matching coworker who also provided usable data. Severe departures from normality, as well as the modest size of the matched sample, provided the impetus for taking a nonparametric (bootstrap) approach.

Results show that task performance is not indirectly related to abusive supervision through any of the proposed mediators. On the other hand, the indirect effects of abusive supervision on OCBOs/OCBIs are significant for all but one mediator (NA). The hypotheses related to CWBOs/CWBIs fared less favorably, such that a significant indirect effect was observed for three of the six mediators (PA, LMX, IJ). When all mediators are included in the same analysis, NA has a significantly stronger indirect effect than AOC. No other significant differences between specific indirect effects are observed.

As expected, PEC showed a negative association with AS and a positive association with the work-related attitudes (LMX, IJ, POS, AOC) proposed to mediate the effects of AS on employee performance. Although PEC moderates the relationship between AS and three of the four work-related attitudes investigated (LMX, IJ, AOC) the direction of these effects is contrary to predictions. An analysis of the nonlinear effects of PEC using a latent variable approach (covariance-based SEM) yields identical findings. Thus, hypotheses regarding the moderating role of PEC are not supported. The status of study hypotheses are listed on Table 31. 


\section{DISCUSSION}

\section{Background and Purpose}

The study of organizational/workplace aggression has evolved considerably over the last few decades and has shed light the relative prevalence and nature of these behaviors. An important theme emerging from this field is that while acts of physical violence (e.g., homicides, battery) represent rare occurrences, subtle forms of aggression (e.g., social undermining, incivility, rudeness) are relatively common in American workplaces (Cortina et al., 2001; Porath \& Pearson, 2013; Schat et al., 2006; SHRM, 2012).

Of interest in the present study is the concept of abusive supervision (AS; Tepper, 2000), which broadly refers to sustained nonphysical (e.g., verbal) aggression from one's immediate supervisor. AS is distinct from similar forms of nonphysical workplace aggression in that it specifies the formal relationship between the perpetrator and target.

The present study advances the AS literature in two ways. First, by integrating across similar mechanisms linking AS (indirectly) to five dimensions of job performancetask performance, citizenship behaviors $(\mathrm{OCBO} / \mathrm{OCBI})$, and counterproductive behaviors $(\mathrm{CWBO} / \mathrm{CWBI}$ - the present study is able to shed light on the relative impact of these related processes. In so doing, it directly answers Tepper et al.'s (2017) call for AS research to incorporate multiple mechanisms under a single mediational framework and evaluate their relative contribution in the same sample. Specifically, the study aims to investigate these various mechanisms using a general sample of US employees. Second, by investigating employee perceptions regarding their firm's EOC as a potential boundary condition vis-à-vis the relationship between $\mathrm{AS}$ and work-related attitudes, the present study not only advances the AS literature, but also extends ethical organizational climate 
theory (Victor \& Cullen, 1988). The remaining sections discuss the findings of this investigation, highlight important limitations arising from aspects of the design and data collection protocol, and offer theoretical and practical implications.

\section{Study Findings}

\section{Primary Findings}

Three sets of hypotheses were formulated with regards to: (1) the indirect effects of AS on subordinate's job performance, (2) the relative contribution (i.e., strength/magnitude) of each specific indirect effect, and (3) the relationships among EOC and AS and the work-related attitudes framed as mediators of the AS-job performance relationship. Employees provided ratings of their work-related attitudes and rated how frequently they experienced AS and affective states during the prior month. Job performance ratings were provided by a coworker whom, in all cases, reported being

familiar with the duties and formal responsibilities of the focal respondent's job and working with the focal respondent on at least a weekly basis. Coworkers that have knowledge of the respondent's formal work role and have regular contact with the respondent are, presumably, more likely to provide valid ratings of the respondent's onthe-job behaviors. That said, the first two sets of hypotheses were tested using the subset of respondents for whom coworker data was available.

AS and Employee Job Performance. The estimated zero-order correlations show significant associations between AS and each employee performance dimension (see Table 7b). AS has a negative association with task performance and citizenship behavior (i.e., OCBO/OCBI) and a positive association with counterproductive behavior (i.e., $\mathrm{CWBO} / \mathrm{CWBI})$. AS is also significantly associated with the affective states and work- 
related attitudes proposed to mediate its effects on performance. Overall, the pattern of correlations among affective states, work-related attitudes, and job performance is consistent with published meta-analytic estimates (see Tables 1 and 2). In line with these estimates, AS shows a stronger association with CWB than with either task performance or OCB.

As noted above, the first set of hypotheses focused on the indirect effects of AS on job performance. Specifically, AS was expected to affect subordinate's job performance indirectly through (1) an decrease in the frequency of positive affective states (PA), (2) an increase in the frequency of negative affective states (PA, NA), (2) its negative effects on leader member exchange (LMX), (3) its negative effects on interactional justice (IJ), (5) its negative effects on affective organizational commitment (AOC), and (6) its negative effects on perceived organizational support (POS). The mediational analyses were somewhat consistent with expectations. Overall, significant indirect effects were observed in $16(53 \%)$ of the 30 single-mediation models tested. These are now discussed, in turn, for each job performance dimension.

Indirect Effects of AS on Employee Job Performance. The hypothesized indirect effects of AS on task performance were not observed for any of the proposed mediators when each was examined separately. The indirect effect of AS on task performance via AOC is observed however, when the mediators were modeled simultaneously. The generally null findings surrounding the indirect effect of AS on task performance suggest attitudinal and affective processes represent poor explanations for the significant negative association observed between AS and task performance. It is possible that the effects of AS on task performance could be due to motivational processes. For instance, the 
experience of AS may push some employees to work harder (and thus escape AS) while pushing others to decrease their work efforts (and thus disengage from work altogether).

The observed indirect effects of AS on contextual performance were generally consistent with expectations. Moreover, each time a specific indirect effect of AS on citizenship and/or counterproductive behavior was observed, findings were consistent across organizational $(\mathrm{OCBO}, \mathrm{CWBO})$ and interpersonal (OCBI, CWBI) dimensions of contextual behavior (see Table 31). AS was indirectly related to OCB through all but one of the proposed mediators (NA) and indirectly related to CWB via PA, LMX, and IJ. When the all mediators are modeled parallel however, the only significant indirect effects of AS on OCB are via NA and PA (OCBO only). Similarly, the indirect effects of AS on CWB are observed via PA (CWBI only) and IJ when modeled in parallel.

Mediation hypotheses supported by the planned analyses were further evaluated to exclude competing explanations. In particular, the observed indirect effects AS on contextual performance through positive affective states (PA) could be explained by trait positive affect (TPA), which predisposes individuals to experience positive affective states. Similarly, it was crucial to show that the observed indirect effects of AS on contextual performance occur through the specific work-related attitudes proposed (i.e., LMX, IJ, AOC, POS), as opposed to work-related attitudes in general. Job satisfaction (JS) refers to “...evaluative judgment one makes about one's job or job situation” (Weiss, 2002, p.175). As an overall evaluation towards multiple aspects of work, JS could pose an alternative explanation for these indirect effects. TPA and JS were included as covariates in analyses involving affect and attitudinal mediators, respectively. 
When the mediation analyses are rerun with the inclusion of relevant covariates, the indirect effects of AS on CWB remain significant. On the other hand, only a third of the indirect effects of AS on OCB - those through LMX and IJ (OCBO only) - remain statistically significant when relevant covariates are included in the model. These findings provide greater confidence in the conclusions reached regarding the indirect effects of AS on CWB. In addition, they highlight the multicollinearity issues that make attempts to discern the specific influence of affective states and conceptually related attitudes a challenging_-yet necessary endeavor.

Relative Contribution of Specific Indirect Effects. An objective of this study was to perform a simultaneous test of the mediators described above, which despite sharing conceptual similarities, had yet to be tested in a manner that allowed their relative contributions to be assessed. This was accomplished by modeling the six mediators, in parallel, and contrasting specific indirect effects pairwise to determine whether they differed in magnitude by a nontrivial amount. Expectations that the specific indirect effects of affective states (PA, NA) would be stronger than the specific indirect effects of supervisor-referenced attitudes (LMX, IJ), which would in turn be stronger than the indirect effects of organization-referenced attitudes (POS, AOC) were generally unsupported by the analyses, which were performed separately for each job dimension (outcome).

Significant differences between specific indirect effects were observed for only three $(5 \%)$ of the sixty pairwise contrasts, and all significant differences involved the contrast between the specific indirect effects of NA and AOC. Specifically, the specific indirect effects of AS on both dimensions of citizenship behaviors (OCBO, OCBI) and on 
CWBI, were stronger for NA than for AOC. The findings regarding citizenship behaviors are surprising given that AOC, but not NA, mediates the relationships between AS and citizenship behaviors (OCBO, OCBI) when these indirect effects are analyzed separately. Overall, results of the pairwise contrast are underwhelming, especially considering the nominal Type 1 error rate for the analyses is also five percent (i.e., null hypotheses rejected at p-values $<.05)$. Ironically, the most likely explanation for the overall absence of significant differences across specific indirect effects is the low statistical power associated with the modest sample size.

Lastly, results of the single and multiple mediation analyses lead to remarkably different conclusions regarding the statistical significance of these indirect effects (see Table 32). Approximately half of the indirect effects that were significant when analyzed in isolation were no longer significant when the indirect effects were modeled as a set. These difference likely stem from the high inter-correlation of affective states and workrelated attitudes. Moreover, they suggest that evidence of mediation presented in prior studies should be interpreted with caution, particularly when competing mechanisms were not included in the analyses.

AS and EOC and Subordinate's Work-related Attitudes. The negative association observed between AS and PEC is consistent with prior research (e.g., Biron, 2010) and with the notion that supervisory behaviors associated with AS are, arguably, more consistent with the behavioral prescriptions from EOCs associated with low PEC (e.g., Instrumental) than from EOCs associated with high PEC (e.g., Caring). The expected positive associations between PEC and each work-related attitude were also observed and 
are consistent with existing research (Mayer, Kuenzi, \& Greenbaum, 2009; Simha \& Cullen, 2012).

Although PEC was found to moderate the relationship between AS and workrelated attitudes, the direction the interactive effect is contrary to predictions. In contrast to predictions, the relationship between AS and AOC is stronger (i.e., more negative) when PEC is high (vs. low). These findings are noteworthy but require an alternative interpretation. It is possible that AS may be more likely to be viewed as being motivated by the supervisor's self-interests when Egoism climates are perceived. On the other hand, EOCs that emphasizes a concern for the well-being of others (e.g., Caring) may lead subordinates to interpret the behaviors associated with AS as "tough love", thus leading to the observed positive relationship between AS and IJ.

\section{Secondary Findings}

This section describes observations regarding the (1) prevalence of AS in the overall sample and across respondent subgroups, (2) consistency between employee- and coworker-rated AS, and (3) viability of operationalizing EOC using the PEC measure. These observations are not specific to any single hypothesis but have a direct bearing on the overall interpretability of the present findings and on the generalizability of study conclusions.

Prevalence of AS. The overwhelming majority of individuals surveyed (70\%) report experiencing AS during the prior 30 days. Items scores further suggest that nonverbal abusive behaviors, such as the failure to give proper credit (52\%) and keep promises (49\%), are more prevalent than verbally abusive behaviors, such as "told me my thoughts

or feeling were stupid" (31\%) and "told me I'm incompetent" (34\%). Moreover, there are 
differences in AS reporting across respondent subgroups based on age, gender, educational attainment, and employment status. Overall, greater AS is reported by respondents whom are male, are under 31 years old (vs. 31 or more), work full-time (vs. part-time), and have greater educational attainment. Combined, these demographic characteristics account for approximately $10 \%$ of the total variance in self-rated AS.

Self- and Peer- rated AS. Measures of AS were rated by focal employees (selfreport) and by their coworkers. Recall that coworkers were asked to rate how frequently they observed AS directed at the focal employee. Although many of the behaviors associated with AS occur in the presence of others, these behaviors vary in their visibility. Whereas abusive behaviors such as ridicule (item \#1), expressions of anger (item \#10), and/or put-downs in front of others (item \#4) are readily observable, behaviors that reference the supervisor's "broken promises" (item \#9) and/or his or her failures to give the subordinate proper credit (item \#7) are somewhat less visible to coworkers.

Although a reasonable degree of consistency across rating sources is expected, ratings of AS provided by the focal employee are virtually indistinguishable from those provided by their coworkers (see Figure 7). Moreover, the degree of consistency between sources is uniform across behaviors which vary in visibility. In other words, focal employees and their coworkers rated abusive behaviors with greater visibility as consistently as they rated abusive behaviors with less visibility. This uniform consistency raises concerns about whether these two sets of ratings were provided by different individuals and suggests that the correlation between employee- and coworker-rated measures of AS shown on Table 6b (Pearson $r=.86$ ) may represent an estimate of consistency across time (e.g., test-retest reliability) rather than across raters. 
Viability of Operationalizing EOC Using PEC. Prior literature reviews note that EOC tends to be operationalize with discrete measures of each climate type (Martin \& Cullen, 2006; Simha \& Cullen, 2012). In contrast, EOC was presently assessed using a single measure-PEC (Hsieh \& Wang, 2016) - purported to reflect employee's global assessment of EOC. The expected higher order structure (five first-order factors plus one second-order factor) was observed—but only after two items (\#19, \#21) were dropped and the residuals between two sets of first-order factors (Independence and Caring, Independence and Instrumental) were allowed to correlate. The present findings suggest that PEC represents a viable option for future research on EOC. Nevertheless, the benefits of assessing EOC along a continuum (i.e., high/low), such as reducing the number of hypotheses (and tests) to be formulated (and evaluated), must be weighed against the risks of obscuring meaningful differences across climate types that may occur as a result collapsing across them.

\section{Limitations}

Confidence in the results of the present study is primarily limited by (1) an inability to establish temporal precedence, (2) artifactual covariance among constructs, and (3) the idiosyncrasies of the sample. The study's cross-sectional design does not support causal claims. To be sure, the use of cross-sectional designs limits the ability to draw causal inferences and is generally condemned by researchers (e.g., Maxwell, Cole, \& Mitchell, 2011) as a means of testing mediation hypotheses. However, considering the lack of guidance currently available regarding the timing of these processes, the added value of anything short of an experimental (or-quasi-experimental) design is debatable (Spector, 2019). Justifications for the proposed temporal precedence among study constructs thus 
rely squarely on theory. Specifically, the present mediational framework is consistent with theoretical arguments that conceptualize (1) AS (e.g., Tepper \& Almeda, 2012), (2) affective states (Affective Events Theory; Weiss \& Cropanzano, 1996), and (3) attitudes (e.g., Theory of Planned Behavior; Ajzen, 1991), as antecedents of employee behaviors.

The second major limitation noted above - the presence of artifactual covariance among study constructs - is a direct result of the data collection protocol. Common method variance (CMV; i.e., variance attributable to the measurement method; Podsakoff, MacKenzie, Lee, \& Podsakoff, 2003) is cause for concerns whenever predictor and criterion measures (1) are obtained from the same individual, (2) contain items that share certain characteristic (e.g., scale formats, anchors), and/or (3) share a common measurement context (e.g., administration medium, location/timing of data collection). CMV due to a common rating source should therefore pose relatively fewer concerns for conclusions related to the indirect effects of AS on employee performance, which utilized data from multiple sources, than for conclusions regarding the effects of PEC, which relied on a single source for all measures.

In contrast to common rater variance, $\mathrm{CMV}$ stemming from the use of similar items and/or a common measurement context is likely to represent a threat to all conclusions. Efforts to reduce respondents' cognitive burden during administration led to using scales with identical format (agreement), number of response alternatives (five), and anchors (strongly disagree-strongly agree) across large sections of the questionnaires. Unfortunately, these efforts may have had the unintended consequence of introducing CMV to the measurement model. CMV generally leads to inflated relationships among constructs, and qualify study conclusions. 
The desire to reach a geographically diverse set of respondents in a cost-effective manner guided most decisions regarding measurement context. Features of the measurement context (e.g., research purposes, administration medium) give rise to additional threats to internal validity. In the relatively low-stakes context of a research study, some respondents may lack the motivation to remain attentive as they complete multiple questionnaire measures. These concerns were addressed however, using survey completion time and instructed items (e.g., Please select never.) scattered throughout the questionnaire to screen out cases that suggested inattentive/low effort responding (based on a priori criteria). Data screening practices have been shown to improve the quality and trustworthiness of survey data (DeSimone \& Harms, 2018).

Relative to administering a questionnaire in the laboratory, remote administration affords researchers less control over basic participant management functions. At times, it may be impossible to determine whether a single individual is the source of multiple questionnaires and/or whether multiple individuals from completed (different parts of) the same questionnaire. Although the compensation protocol was specifically designed discourage multiple entries from the same individual, it is far from a guarantee that each case in the present dataset represents a unique participant.

Moreover, the uniform consistency observed across employee- and coworker-rated AS items (see above) calls into question the proportion of respondent who followed study instructions to recruit a coworker relative to those whom may have elected to complete the coworker questionnaire themselves. While the inability to ascertain the independence of data from ostensibly different sources threaten conclusions regarding employee 
performance, it bears little influence on findings related to the effects of PEC, which by design, involved only self-rated constructs.

The third major limitation to study conclusions arises from characteristics of the recruitment platform and study participants. Recall that study participants were recruited using Amazon's Mechanical Turk (MTurk). Investigations regarding the viability of conducting psychological research on MTurk conclude that the data quality is comparable to undergraduate students (e.g., Behrend, Sharek, Meade, \& Wiebe, 2011) and to data collected from other internet sources (e.g., Buhrmester, Kwang, \& Gosling, 2011). MTurk has also been shown to provide more diverse samples than university subject pools (Behrend et al., 2011; Buhrmester, Kwang, \& Gosling, 2011; Gosling, Vazire, Srivastava \& John, 2004). Moreover, Highhouse and Zhang (2015) note that in comparison with university subject pool samples, samples collected on MTurk samples are more similar to organizational samples and thus preferable in terms of their generalizability.

Nevertheless, the unique characteristics of study participants threaten the generalizability of conclusions to the intended population of interest (i.e., full and part-time employees in the US). Despite approximating the US labor force in terms of gender and race (see Table 5; note that the BLS assesses Hispanic ethnicity separately from race), participants in the study are markedly younger (sample median age $=31$ vs. 42 in labor force) and better educated ( $45 \%$ vs. $25 \%$ have college degree). Participants represent all 20 major industrial sectors. Lastly, some industries are overrepresented (e.g., Finance \& Insurance, Information, Management) and others are underrepresented (e.g., Agriculture, Forestry, Fishing \& Hunting) in relation to their levels in the labor force. These sample 
characteristics suggest the present findings should be generalized only to US employees whom are relatively young, highly educated, and hold "office jobs."

\section{Implications and Future Directions}

Undoubtedly, organizations should be committed to the prevention and reduction of AS. It is clear from the present study and from prior research in this area (see Tepper et al., 2017), that AS is both prevalent in American workplaces and that its effects on employee attitudes and work behaviors are largely detrimental. The present study suggests however, that in addition to focusing on the actor's behavior, organizations should be mindful of the ethical climate created and perpetuated by their leadership staff and/or implied by their policies. Interestingly, the present research shows that under some EOCs, abused employees may nonetheless hold favorable work attitudes. That said, interventions targeting organizational climate may yield favorable results in efforts to reduce the detrimental effects of supervisory abuse. Finally, in light of the numerous ways in which AS appears to impact employee performance, human resource practitioner may find it worthwhile to develop interventions which, in addition to targeting the source of abusive supervision (i.e., the supervisor's hostility), also target multiple employee work attitudes.

The present findings also highlight subgroup differences regarding the prevalence of AS and suggest that abuse is not evenly distributed among employees. Indeed, male employees, as well as younger and more educated employees tend to report a higher degree of AS than their counterparts. Moreover, corroborating data from coworkers suggests these subgroup differences are not merely due to differences in the perception of abuse or the result of reporting biases. These findings imply that organizations may find value in targeting specific employee groups for interventions. 
The mediators hypothesized to explain the effects of AS on employee performance performed somewhat better against contextual performance than against task performance. While many of the hypothesized indirect effects of AS on contextual performance were supported by the data, there was an overall failure to detect significant difference across the various mechanisms. The most probable explanation, as noted earlier, concerns the lack of statistical power. On this note, researchers seeking to use MTurk as a research platform should be cognizant of its limitation with regards to multi-source samples. For instance, less than a third of the more than 8,500 individuals whom initially agreed to participate actually provided usable data. Further, only a tenth of the individuals whom provided usable data recruited an eligible coworker. While the present study benefits from ecological validity by virtue of a large heterogenous sample, future research may benefit from the use of experimental designs. In particular, there is a clear need to establish the temporal primacy of abusive supervision and/or abusive actions in shaping employee behaviors.

Although this study conceptualized all mediators operating in parallel, future studies may benefit from exploring sequential mediator models. Indeed, AET (Weiss \& Cropanzano, 1996) specifically suggests that the behavioral effects of affective states are further mediated by work attitudes. It should be noted however, that the present lack of understanding regarding the tempo of affective states and attitude formation resulting from AS continues to be an impediment to testing sequential models. That said, more research is clearly needed to determine the timing of the psychological processes currently investigated.

Lastly, findings regarding the interactive effects of AS and PEC need to be explored in future research. At the present, these observations suggest that when EOCs emphasize a 
concern for others, increasing levels of AS are associated with more favorable perceptions of IJ. As suggested, this could be due to a tendency on the part of some subordinates to view these behaviors as an indication of their supervisor's concern towards them. Supervisors vary in the extent to which they view AS instrumentally (i.e., to improve employee outcomes) and these beliefs predict engagement in AS (Watkins, Fehr, \& He, in press). Thus, investigation regarding the role of EOC (if any) in shaping supervisor's AS instrumentality beliefs represents a viable avenue for future research. 


\section{REFERENCES}

Allen, N. J., \& Meyer, J. P. (1990). The measurement and antecedents of affective, continuance and normative commitment to the organization. Journal of Occupational Psychology, 63(1), 1-18.

Amazon.com. (2016). Mechanical Turk, Retrieved June 2016 from http://www.mturk.com/.

Anderson, C. A., \& Bushman, B. J. (2002). Human aggression. Annual Review of Psychology, 53, 27-51.

Anderson, J. C., \& Gerbing, D. W. (1984). Structural equation modeling in practice: A review and recommended two-step approach. Psychological Bulletin, 103(3), 411-423.

Andersson, L. M., \& Pearson, C. M. (1999). Tit for tat? The spiraling effect of incivility in the workplace. Academy of Management Review, 24(3), 452-471.

Aquino, K., \& Bradfield, M. (2000). Perceived victimization in the workplace: The role of situational factors and victim characteristics. Organization Science, 11(5), 525-537.

Aquino, K., \& Thau, S. (2009). Workplace victimization: Aggression from the target's perspective. Annual Review of Psychology, 60, 717-741.

Doi:10.1146/annurev.psych.60.110707.163703.

Aryee, S., Chen, Z. X., Sun, L., \& Debrah, Y. A. (2007). Antecedents and outcomes of abusive supervision: Test of a trickle-down model. Journal of Applied Psychology, 92(1), 191-201.

Aryee, S., Sun, L. Y., Chen, Z. X. G., \& Debrah, Y. A. (2008). Abusive supervision and contextual performance: The mediating role of emotional exhaustion and the moderating role of work unit structure. Management and Organization Review, 4(3), 393-411.

Atwater, L., Kim, K. Y., Witt, A., Latheef, Z., Callison, K., Elkins, T. J., \& Zheng, D. (2016). Reactions to abusive supervision: Examining the roles of emotions and gender in the usa. The International Journal of Human Resource Management, 27(16), 1874-1899. DOI:10.1080/09585192.2015.1088887.

Avey, J. B., Wu, K., \& Holley, E. (2015). The influence of abusive supervision and job embeddedness on citizenship and deviance. Journal of Business Ethics, 129(3). 721-731.

Ajzen, I. (2001). Nature and operation of attitudes. Annual Review of Psychology, 52, $27-$ 58.

Baillen, E., Escartin, J., Gross, C., \& Zapf, D. (2017). Towards a conceptual and empirical differentiation between workplace bullying and interpersonal conflict, 
European Journal of Work and Organizational Psychology, 26(6). 870-881.

Doi:10.1080/1359432X.2017.1385601.

Bamberger, P. A., \& Bacharach, S. B. (2006). Abusive supervision and subordinate problem drinking: Taking resistance, stress and subordinate personality into account. Human Relations, 59(6), 723-752. Doi:10.1177/0018726706066852.

Barling, J., Dupre, K. E., \& Kelloway, E. K. (2009). Predicting workplace aggression and violence. Annual Review of Psychology, 60, 671-692.

Barnet, T. \& Vaicys, C. (2000). The moderating effect of individuals' perception of ethical work climate on ethical judgments and behavioral intentions. Journal of Business Ethics, 27(4), 351-362.

Baron, R. M., \& Kenny, D. A. (1986). The moderator-mediator variable distinction in social psychological research: Conceptual, strategic, and statistical considerations.

Journal of Personality and Social Psychology, 51(6), 1173-1182.

Barsky, A. \& Kaplan, S. A. (2007). If you feel bad, it's unfair: A quantitative synthesis of affect and organizational justice perceptions. Journal of Applied Psychology, 92(1). 286295.

Bauer, J.A. \& Spector, P. E. (2015). Discrete negative emotions and counterproductive work behavior. Human Performance, 28, 307-331. Doi:

10.1080/08959285.2015.1021040.

Beal, D. J., Weiss, H. M., Barros, E., \& MacDermid, S. M. (2005). An episodic process model of affective influences on performance. Journal of Applied Psychology, 90(6), 1054-1068. Doi:10.1037/0021-9010.90.6.1054.

Behrend, T. \& Sharek, D., Meade, A., \& Wiebe, E., (2011). The viability of crowdsourcing for survey research. Behavior Research Methods, 43, 800813.Doi:10.3758/s13428-011-0081-0.

Berdahl, J. L. (2007). Harassment based on sex: Protecting social status in the context of gender hierarchy. The Academy of Management Review, 32(2), 641-658.

DOI:10.5465/AMR.2007.24351879.

Berry, C.M., Ones, D.S., \& Sackett, P.R. (2007). Interpersonal deviance, organizational deviance, and their common correlates: A review and meta-analysis. Journal of Applied Psychology, 92(2). 410-424. Doi:10.1037/0021-9010.92.2.410.

Biron, M. (2010). Negative reciprocity and the association between perceived organizational ethical values and organizational deviance. Human Relations, 63(6), 875897. 
Bollman, G., \& Krings, F. (2016). Workgroup climates and employees' counterproductive work behaviors: A social-cognitive perspective. Journal of Management, 53(2). 184-209. Doi:10.1111/ joms.12167.

Bowling, N. A., \& Beehr, T. A. (2006). Workplace harassment from the victim's perspective: A theoretical model and meta-analysis. Journal of Applied Psychology, 91(5), 998-1012. Doi:10.1037/0021-9010.91.5.998.

Bowling, N. A., \& Michel, J. S. (2011). Why do you treat me badly? The role of attributions regarding the cause of abuse in subordinates' responses to abusive supervision. Work \& Stress, 25(4), 309-320. Doi:10.1080/02678373.2011.634281.

Brown, M. E., Treviño, L. K., \& Harrison, D. A. (2005). Ethical leadership: A social learning perspective for construct development and testing. Organizational Behavior and Human Decision Processes, 97(2), 117-134.

Buhrmester, M., Kwang, T., \& Gosling, S. D. (2011). Amazon's mechanical turk: A new source of inexpensive, yet high-quality, data. Perspectives on Psychological Science, $6(1), 3-5$.

Bulutlar, F., \& Oz, E. U. (2009). The effects of ethical climate on bullying behavior in the workplace. Journal of Business Ethics, 86(3). 273-295.

Cain, M. K., Zhang, Z., \& Yuan, K. (2016). Univariate and multivariate skewness and kurtosis for measuring nonnormality: Prevalence, influence and estimation. Behavior Research Methods, 49(5). 1716-1735.

Carlson, D. S., Ferguson, M., Hunter, E., \& Whitten, D. (2012). Abusive supervision and work-family conflict: The path through emotional labor and burnout. The Leadership Quarterly, 23(5). 849-859.

Carlson, D. S., Ferguson, M., Perrewé, P. L., \& Whitten, D. (2011). The fallout from abusive supervision: An examination of subordinates and their partners. Personnel Psychology, 64(4), 937-961.

Carver, C. S., \& Harmon-Jones, E. (2009). Anger is an approach related affect: Evidence and implications. Psychological Bulletin, 135(2). 183-204.

Cetin, S. Gurbuz, S, \& Sert, M. (2015). A meta-analysis of the relationship between organizational commitment and organizational citizenship behavior: Test of potential moderator variables. Employee Responsibility and Rights Journal, 27(4). 281-303. Doi:10.1007/s10672-015-9266-5.

Chan, M. E., \& McAllister, D. J. (2014). Abusive supervision through the lens of employee state paranoia. The Academy of Management Review, 39(1), 44-66. 
Chu, L. C. (2014). Mediating toxic emotions in the workplace: The impact of abusive supervision. Journal of Nursing Management, 22(8), 953-963.

Cohen, J. (1988). Statistical power analysis for the behavioral sciences (2e.). Hillsdale, NJ: Erlbaum.

Cohen-Charash, Y., \& Spector, P. E. (2001). The role of justice in organizations: A metaanalysis. Organizational Behavior and Human Decision Processes, 86(2), 278-321.

Coleman, V., \& Borman, W.C. (2000). Investigating the underlying structure of the citizenship performance domain. Human Resource Management Review, 10(1). 25-44.

Collins, M. D., \& Jackson, C. J. (2015). A process of self-regulation and leadership: How attentional resource capacity and negative emotions influence constructive and destructive leadership. The Leadership Quarterly, 26(3). 386-401.

Doi:10.1016/j.leaqua.2015.02.005.

Colquitt, J. A. (2001). On the dimensionality of organizational justice: A construct validation of a measure. Journal of Applied Psychology, 86(3), 386-400.

Colquitt, J. A., Scott, B. A., Rodell, J. B., Long, D. M., Zapata, C. P., Conlon, D. E., \& Wesson, M. J., (2013). Justice at the millennium, a decade later: A meta-analytic test of social exchange and affect-based perspectives. Journal of Applied Psychology, 98(2), 199-236. Doi:10.1037/a0031757

Cortina, L. M., \& Magley, V. J. (2003). Raising voice, risking retaliation: Events following interpersonal mistreatment in the workplace. Journal of Occupational Health Psychology, 8(4). 247-265. Doi:10.1037/1076-8998.8.4.247.

Cortina, L. M., Magley, V. J., Williams, J. H., \& Langhout, R. D. (2001). Incivility in the workplace: Incidence and impact. Journal of Occupational Health Psychology, 6(1), 6480. Doi:10.1037//1076-8998.6.1.64.

Cullen, J. B., Parboteeah, K. P., \& Victor, B. (2003). The effects of ethical climates on organizational commitment: A two-study analysis. Journal of Business Ethics, 46(2), 127-141.

Cullen, J. B., Victor, B., \& Bronson, J. W. (1993). The ethical climate questionnaire: An assessment of its development and validity. Psychological Reports, 73(2), 667-674.

Dalal, R. S. (2005). A meta-analysis of the relationship between organizational citizenship behavior and counterproductive work behavior. Journal of Applied Psychology, 90(6), 1241-1255. Doi:10.1037/0021-9010.90.6.1241.

Dalal, R. S., Lam, H., Weiss, H. W., Welch, E. B., \& Hulin, C. L. (2009). A withinperson approach to work behavior and performance: Concurrent and lagged citizenship- 
counterproductivity associations, and dynamic relationships with affect and overall job performance. The Academy of Management Journal, 52(5), 1051-1066.

DeCarlo, L. T. (1997). On the meaning and use of kurtosis. Psychological Methods, 2(3), 292-307.

DeConinck, J. B. (2011). The effects of ethical climate on organizational identification, supervisory trust, and turnover among salespeople. Journal of Business Research, 64(6), 617-624. Doi:10.1016/j.jbusres.2010.06.014.

Decoster, S., Camps, J., \& Stouten, J. (2014). The mediating role of LMX between abusive supervision and work behaviors: A replication and extension. American Journal of Business, 29(1). 61-75.

Detert, J. R., Treviño, L. K., Burris, E. R., \& Andiappan, M. (2007). Managerial modes of influence and counterproductivity in organizations: A longitudinal business-unit-level investigation. Journal of Applied Psychology, 92(4): 993-1005.

Duffy, M. K., Ganster, D., \& Pagon, M. (2002). Social undermining and social support in the workplace. The Academy of Management Journal, 45(2). 331-351.

Eagly, A. H., \& Chaiken, S. (1993). The psychology of attitudes. Orlando, FL, US: Harcourt Brace Jovanovich College Publishers.

Eisenberger, R. Cummings, J., Armeli, S., \& Lynch, P. D. (1997). Perceived organizational support, discretionary treatment, and job satisfaction. Journal of Applied Psychology, 82(5), 812-820.

Eisenberger, R., Armeli, S., Rexwinkel, B., Lynch, P. D., \& Rhoades, L. (2001). Reciprocation of perceived organizational support. Journal of Applied Psychology, 86(1), 42-51.

Eisenberger, R., Huntington, R., Hutchison, S., \& Sowa, D. (1986). Perceived organizational support. Journal of Applied Psychology, 71(3), 500-507.

Eisenberger, R., Karagonlar, G., Stinglhamber, F., Neves, P., Becker, T. E., GonzalezMorales, M. G., \& Steiger-Mueller, M. (2010). Leader-member exchange and affective organizational commitment: The contribution of supervisor's organizational embodiment. Journal of Applied Psychology, 95(6), 1085-1103. DOI: 10.1037/a0020858.

Erez, A., \& Isen, A. M. (2002). The influence of positive affect on the components of expectancy motivation. Journal of Applied Psychology, 87(6). 1055-1067.

Estes, B., \& Wang, J. (2008). Integrative literature review: Workplace incivility: Impacts on individual and organizational performance. Human Resource Development Review, $7(2), 218-240$. 
Fisher, C. D. (2002). Antecedents and consequences of real-time affective reactions at work. Motivation and Emotion, 26 (1), 3-30.

Fox, S., Spector, P. E., Goh, A., Bruursema, K., \& Kessler, S. R. (2009). The deviant citizen: Clarifying the measurement of organizational citizenship behavior and its relation to counterproductive work behavior. Loyola University Chicago.

Fritz, M.S., Taylor, A.B. \& MacKinnon, D.P. (2012). Explanation of two anomalous results in statistical mediation analysis. Multivariate Behavioral Research, 47 (1), 61-87.

George, J. M. (1991). State or trait: Effects of positive mood on prosocial behaviors at work. Journal of Applied Psychology, 76(2), 299-307.

George, J. M., \& Zhou, J. (2002). Understanding when bad moods foster creativity and good ones don't: The role of context and clarity of feelings. Journal of Applied Psychology, 87(4), 687-697.

Gerstner, C. R., \& Day, D. V. (1997). Meta-analytic review of leader-member exchange theory: Correlates and construct issues. Journal of Applied Psychology, 82(6), 827-844.

Gosling, S. D., Vazire, S., Srivastava, S., \& John, O. P. (2004). Should we trust webbased studies? A comparative analysis of six preconceptions about internet questionnaires. American Psychologist, 59(2), 93-104.

Graen, G. B., \& Uhl-Bien, M. (1995). Relationship-based approach to leadership: Development of leader-member exchange $(\operatorname{lmx})$ theory of leadership over 25 years: Applying a multi-level multi-domain perspective. The Leadership Quarterly, 6(2), 219247.

Graen, G., \& Schiemann, W. (1978). Leader-member agreement: A vertical dyad linkage approach. Journal of Applied Psychology, 63(2). 206-212.

Greenberg, L., \& Barling, J. (1999). Predicting employee aggression against coworkers, subordinates and supervisors: The roles of person behaviors and perceived workplace factors. Journal of Organizational Behavior, 20(6), 897-913.

Grojean, M. W., Resick, C. J., Dickson, M. W., \& Smith, D. B. (2004). Leaders, values, and organizational climate: Examining leadership strategies for establishing an organizational climate regarding ethics. Journal of Business Ethics, 55(3), 223-241.

Harris, K. J., Kacmar, K. M., \& Zivnuska, S. (2007). An investigation of abusive supervision as a predictor of performance and the meaning of work as a moderator of the relationship. The Leadership Quarterly, 18(3), 252-263.

Harvey, P., Stoner, J., Hochwarter, W., \& Kacmar, C. (2007). Coping with abusive supervision: The neutralizing effects of ingratiation and positive affect on negative employee outcomes. The Leadership Quarterly, 18(3), 264-280. 
Hayes, A. F. (2012). PROCESS: A versatile computational tool for observed variable mediation, moderation, and conditional process modeling [White paper]. Retrieved June 2018 from http://www.afhayes.com/public/process2012.pdf.

Hayes, A. F. (2017). Introduction to mediation, moderation, and conditional process analysis, second edition: A regression-based approach (2e). Guilford Press. New YorkLondon.

Hershcovis, M. S. (2011). "Incivility, social undermining, bullying. . Oh my!": A call to reconcile constructs within workplace aggression research. Journal of Organizational Behavior, 32(3), 499-519. DOI:10.1002/job.689.

Hershcovis, M. S., \& Barling, J. (2010). Towards a multi-foci approach to workplace aggression: A meta-analytic review of outcomes from different perpetrators. Journal of Organizational Behavior, 31(1), 24-44. Doi:10.1002/job.621.

Hoobler, J.M., \& Hu, J. (2013). A model of injustice, abusive supervision, and negative affect. The Leadership Quarterly, 24(1), 256-69.

Hsieh, H., \& Wang, Y. (2016). Linking perceived ethical climate to organizational deviance: The cognitive, affective, and attitudinal mechanism. Journal of Business Research, 69(9). 3600-3608. Doi:10.1016/j.jbusres.2016.01.001.

Hussain, I., \& Sia, S. K. (2017). Power distance orientation dilutes the effect of abusive supervision on workplace deviance. Management and Labour Studies, 42(4), 1-13. Doi:10.1177/0258042X17731981.

Iacobucci, D. (2009) Everything you always wanted to know about sem (structural equations modeling) but were afraid to ask. Journal of Consumer Psychology. 19(4). 673680.

Ilies, R., Nahrgang, J. D., \& Morgeson, F. P. (2007). Leader-member exchange and citizenship behaviors: A meta-analysis. Journal of Applied Psychology, 92(1), 269-277.

Inness, M., LeBlanc, M. M., \& Barling, J. (2008). Psychosocial predictors of supervisor-, peer-, subordinate-, and service-provider-targeted aggression. Journal of Applied Psychology, 93(6), 1401-1411.

Jackson, D. L. (2001). Sample size and number of parameter estimates in maximum likelihood confirmatory factor analysis: A monte carlo investigation. Structural Equation Modeling, 8(2), 205-223.

Jian, Z., Kwan, H. K., Qiu, Q., Liu, Z. Q., \& Kim, F. H.-K. (2012). Abusive supervision and frontline employees' service performance. The Service Industries Journal, 32(5): 683-698. 
Jöreskog, K.G. \& Sörbom, D. (2018). LISREL 10 [Computer software]. Scientific Software International, Inc. Chicago, IL.

Judge, T. A., \& Kammeyer-Mueller, J. D. (2012). Job attitudes. Annual Review of Psychology, 63, 341-367.

Judge, T. A., Scott, B. A., \& Ilies, R. (2006). Hostility, job attitudes, and workplace deviance: Test of a multilevel model. Journal of Applied Psychology, 91(1), 126-138.

Kaplan, S., Bradley, J. C., Luchman, J. N., \& Haynes, D. (2009). On the role of positive and negative affectivity in job performance: A meta-analytic investigation. Journal of Applied Psychology, 94(1), 162-176. Doi: 10.1037/a0013115.

Keashly, L. (1997). Emotional abuse in the workplace: Conceptual and empirical issues. Journal of Emotional Abuse, 1(1), 85-117.

Keashly, L., Trott, V., \& MacLean, L. M. (1994). Abusive behavior in the workplace: A preliminary investigation. Violence and Victims, 9(4): 341-357.

Khan, A. K., Moss, S., Quratulain, S., \& Hameed, I. (2018). When and how subordinate performance leads to abusive supervision: A social dominance perspective. Journal of Management, 44(7), 2801-2826. Doi:10.1177/0149206316653930.

Khan, A. K., Quratulain, S., \& Crawshaw, J. R. (2013). The mediating role of discrete emotions in the relationship between injustice and counterproductive work behaviors: A study in pakistan. Journal of Business Psychology, 28(1), 49-61.

Klaussner, S. (2014). Engulfed in the abyss: The emergence of abusive supervision as an escalating process of supervisor-subordinate interaction. Human Relations, 67(3), 311332. Doi:10.1177/0018726713493027.

Klein, K. J., \& Sorra, J. S. (1996). The challenge of innovation implementation. Academy of Management Review, 21(4), 1055-1080.

Kline, R. B. (2011). Principles and practice of structural equation modeling (3e). Guilford Press. New York, NY.

Kohlberg, L. (1984) The psychology of moral development: The nature and validity of moral stages. V.2. Harper-collins. College Div.

Kung, F. Y., Kwok, N., \& Brown, D. J. (2018). Are attention check questions a threat to scale validity? Applied Psychology: An International Review. 67(2). 264-283.

Lee, K., \& Allen, N. J. (2002). Organizational citizenship behavior and workplace deviance: The role of affect and cognitions. Journal of Applied Psychology, 87(1), 131142. 
Lee, S., Yun, S., \& Srivastava, A. (2013). Evidence for a curvilinear relationship between abusive supervision and creativity in south korea. The Leadership Quarterly, 24(5), 724731.

Leung, A. S. (2008). Matching ethical work climate to in-role and extra-role behaviors in a collectivistic work setting. Journal of Business Ethics, 79(1/2), 43-55.

Doi:10.1007/s10551-007-9392-6.

Leymann, H. (1996). The content and development of mobbing at work. European Journal of Work and Organizational Psychology 5(2), 165-184.

DOI:10.1080/13594329608414853.

Li, Y., Wang, Z., Yang L. Q., \& Liu, S. (2016). The crossover of psychological distress from leaders to subordinates in teams: the role of abusive supervision, psychological capital, and team performance. Journal of Occupational Health Psychology, 21(2), 142153.

Lian H., Ferris D. L., Morrison, R., \& Brown, D. J. (2014). Blame it on the supervisor or the subordinate? Reciprocal relations between abusive supervision and organizational deviance. Journal of Applied Psychology, 99(4), 651-664.

Lian H., Ferris, D. L., \& Brown, D. J. (2012b). Does taking the good with the bad make things worse? How abusive supervision and leader-member exchange interact to impact need satisfaction and organizational deviance. Organizational Behavior and Human Decision Processes, 117(1), 41-52.

Lian, H., Ferris, D. L., \& Brown, D. J. (2012a). Does power distance exacerbate or mitigate the effects of abusive supervision? It depends on the outcome. Journal of Applied Psychology, 97(1), 107-123.

Liao, H., \& Rupp, D. E. (2005). The impact of justice climate and justice orientation on work outcomes: A cross-level multifoci framework. Journal of Applied Psychology, 90(2), 242-256. Doi:10.1037/0021-9010.90.2.242.

Lin, S., Ma, J., \& Johnson, R. E. (2016). When ethical leader behavior breaks bad: how ethical leader behavior can tuÍrn abusive via ego depletion and moral licensing. Journal of Applied Psychology, 101(6), 815-830.

Lovett, M., Bajaba, S. Lovett, M., \& Simmering, M.J., (2018). Data quality from crowdsourced surveys: A mixed method inquiry into perceptions of amazon's mechanical turk masters. Applied Psychology: An International Review. 67(2). 339-366.

Lyons, B. J., \& Scott, B. A., (2012). Integrating social exchange and affective explanations for receipt of help and harm: A social network approach. Organizational Behavior and Human Decision Processes, 117(1), 66-79.

Doi:10.1016/j.obhdp.2011.10.002. 
Lyu, Y., Zhum H., Zhong, H., \& Hu, L. (2016). Abusive supervision and customeroriented organizational citizenship behavior: The roles of hostile attribution bias and work engagement. Journal of Hospitality Management, 53, 69-80.

Mackey, J. D., Frieder, R. E., Brees, J. R., \& Martinko, M. J. (2017). Abusive supervision: A meta-analysis and empirical review. Journal of Management, 43(6), 19401965.

MacKinnon, D. P., Lockwood, C. M., Hoffman, J. M., West, S. G., \& Sheets, V. (2002). A comparison of methods to test mediation and other intervening variable effects. Psychological Methods, 7(1), 83-104.

MacKinnon, D. P., Warsi, G., \& Dwyer, J. H. (1995). A simulation study of mediated effect measures. Multivariate Behavioral Research, 30(1), 41-62.

Malatesta, R. M., \& Byrne, Z. S. (1997). The impact of formal and interactional procedures on organizational outcomes. Paper presented at the 12th annual conference of the Society for Industrial and Organizational Psychology, St. Louis, MO.

Mardia, K.V., Kent, J.T., \& Bibby, J.M., (1979). Multivariate Analysis. Academic Press, London.

Martin, K. \& Cullen, J. (2006). Continuities and extensions of ethical climate theory: A meta analytic review. Journal of Business Ethics 69(2), 175-194. Doi:10.1007/s10551006-9084-7.

Martin, R., Guillaume, Y., Thomas, G., Lee, A., \& Epitropaki, O. (2016). Leadermember exchange $(\operatorname{lmx})$ and performance: A meta-analytic review. Personnel Psychology, 69(1). 67-121. Doi:10.1111/peps.12100.

Martinko, M. J., Harvey, P., Brees, J. R., \& Mackey, J. (2013). A review of abusive supervision research. Journal of Organizational Behavior, 34(S1), 120-137.

Martinko, M. J., Harvey, P., Sikora, D., \& Douglas, S. C. (2011). Perceptions of abusive supervision: The role of subordinates' attribution styles. The Leadership Quarterly, 22(4), 751-764.

McColl-Kennedy, J. R., \& Anderson, R. D. (2002). Impact of leadership style and emotions on subordinate performance. The Leadership Quarterly, 13(5), 545-559.

Meyer, J. P., Paunonen, S. V., Gellatly, I. R., Goffin, R. D., \& Jackson, D. N. (1989). Organizational commitment and job performance: It's the nature of the commitment that counts. Journal of Applied Psychology, 74(1), 152-156.

Meyer, J. P., Stanley, D. J., Herscovitch, L., \& Topolnytsky, L. (2002). Affective, continuance, and normative commitment to the organization: A meta-analysis of 
antecedents, correlates, and consequences. Journal of Vocational Behavior, 61(1), 20-52. Doi:10.1006/jvbe.2001.1842.

Michel, J. S., Newness, K., \& Duniewicz, K. (2016). How abusive supervision affects workplace deviance: A moderated-mediation examination of aggressiveness and workrelated negative affect. Journal of Business and Psychology, 31(1). 1-22.

Mikula, G. (1993). On the experience of injustice. European Review of Social Psychology, 4(1).223-244. Doi:10.1080/14792779343000077.

Miles, D. E., Borman, W. E., Spector, P. E., \& Fox, S. (2002). Building an integrative model of extra-role work behaviors: A comparison of counterproductive work behavior with organizational citizenship behavior. International Journal of Selection and Assessment, 10(1/2), 51-57.

Miner, A. G., \& Glomb, T. M. (2010). State mood, task performance, and behavior at work: A within-person approach. Organizational Behavior and Human Decision Processes, 112(1), 43-57. Doi:10.1016/j.obhdp.2009.11.009.

Mitchell, M. S., \& Ambrose, M. L. (2007). Abusive supervision and workplace deviance and the moderating effects of negative reciprocity beliefs. Journal of Applied Psychology, 92(4), 1159-1168. Doi:10.1037/0021-9010.92.4.1159.

Moorman, R. H. (1991). Relationship between organizational justice and organizational citizenship behaviors: Do fairness perceptions influence employee citizenship? Journal of Applied Psychology, 76(6), 845-855.

Motowidlo, S. J., \& van Scotter, J. R. (1994). Evidence that task performance should be distinguished from contextual performance. Journal of Applied Psychology, 79(4), 475480 .

Mulki, J. P., Jaramillo, F., \& Locander, W. B. (2006). Effects of ethical climate and supervisory trust on salesperson's job attitudes and intentions to quit. Journal of Personal Selling \& Sales Management, 26(1), 19-26.

Muraven, M., \& Baumeister, R. F. (2000). Self-regulation and depletion of limited resources: Does self-control resemble a muscle? Psychological Bulletin, 126(2), 247-259.

Nandkeolyar, A. K., Shaffer, J. A., Li, A., Ekkirala, S., \& Bagger, J. (2014). Surviving an abusive supervisor: the joint roles of conscientiousness and coping strategies. Journal of Applied Psychology, 99(1), 138-150.

Neubert, M.J., Carlson, D. S., Kacmar, K. M., Roberts, J. A., \& Chonko, L. B. (2009). The virtuous influence of ethical leadership behavior: Evidence from the field. Journal of Business Ethics, 90(2), 157-170. Doi:10.1007/s10551-009-0037-9. 
Neuman, J.H., \& Baron, R.A., (1998). Workplace violence and workplace aggression: Evidence of specific forms, potential causes, and preferred targets. Journal of Management 24(3), 391-419.

Nixon, A. E., \& Spector, P.E. (2015). Seeking clarity in a linguistic fog: Moderators of the workplace aggression-strain relationship. Human Performance, 28(2). 137-164. Doi:10.1080/08959285.2015.1006325.

O*NET (2018). Work activities. National Center for $O^{*}$ NET Development. Retrieved June 2018 from https://www.onetonline.org/find/descriptor/browse/Work_Activities/.

Oh, J. K., \& Farh, C. I. C. (2017). An emotional process theory of how subordinates appraise, experience, and respond to abusive supervision over time. The Academy of Management Review, 42(2), 207-232. Doi:10.5465/amr.2014.0347.

O'Leary-Kelly, A. M., Griffin, R. W., \& Glew, D. J. (1996). Organization-motivated aggression: A research framework. The Academy of Management Review, 21(1), $225-$ 253.

Oncale v. Sundowner Offshore Service, Inc. (October, 1997): US court of appeals: $5^{\text {th }}$ district. Case summary. Retrieved June 2018 from https://supreme.justia.com/cases/federal/us/523/75/case.pdf.

Peng, A. C., Schaubroeck, J. M., \& Li, Y. (2014). Social exchange implications of own coworkers' experiences of supervisory abuse. The Academy of Management Journal, 57(5). 1385-1405.

Peng, A. C., Schaubroeck, J. M., Chong, S., \& Li, Y. (in press). Discrete emotions linking abusive supervision to employee intention and behavior. Personnel Psychology. Doi:10.1111/peps.12310.

Penney, L. M., \& Spector, P. E. (2005). Job stress, incivility, and counterproductive work behavior (cwb): The moderating role of negative affectivity. Journal of Organizational Behavior, 26(7). 777-796.

Petty, R. E., Wegener, D. T., \& Fabrigar, L.R., (1997). Attitudes and attitude change. Annual Review of Psychology, 48. 609-647.

Podsakoff, N., Whiting, S.W., Podsakoff, P. M., \& Blume, B. D. (2009). Individual and organization-level consequences organizational citizenship behaviors: A meta-analysis. Journal of Applied Psychology, 94(1), 122-141.

Porath, C. L., \& Pearson, C. (2013). The price of incivility: Lack of respect hurts morale - and the bottom line. Harvard Business Review. Retrieved June 2018 from https://hbr.org/2013/01/the-price-of-incivility. 
Porath, C. L., \& Erez, A. (2007). Does rudeness really matter? The effects of rudeness on task performance and helpfulness. The Academy of Management Journal, 50(5), 11811197.

Porath, C., L., MacInnis, D., \& Folkes, V. S. (2011). Its unfair: Why customers who merely observe an uncivil employee abandon the company. Journal of Service Research, 14(3), 302-317. Doi:10.1177/1094670511404393.

Preacher, K. J., \& Hayes, A. F. (2008). Asymptotic and resampling strategies for assessing and comparing indirect effects in multiple mediator models. Behavior Research Methods, 40(3). 879-891.

Preacher, K. J., \& Hayes, A. F. (2004) Spss and sas procedures for estimating indirect effects in simple mediation models. Behavior Research Methods, Instruments, \& Computers, 36(4), 717-731.

Priesemuth M., Schminke M., Ambrose, M. L., \& Folger, R. (2014). Abusive supervision climate: A multiple-mediation model of its impact on group outcomes. The Academy of Management Journal, 57(5), 1513-1534.

Rafferty, A. E., \& Restubog, S. L. D. (2011). The influence of abusive supervisors on followers' organizational citizenship behaviours: The hidden costs of abusive supervision. British Journal of Management, 22(2), 270-285.

Razali, N.M., \& Wah, Y.B., (2011). Power comparisons of shapiro-wilk, kolmogorovsmirnov, liliefors, and anderson-darling tests. Journal of Statistical Modeling and Analytics, 2(1). 21-33.

Restubog, S. L. D., Scott, K. L., \& Zagenczyk, T. J. (2011). When distress hits home: The role of contextual factors and psychological distress in predicting employees' responses to abusive supervision. Journal of Applied Psychology, 96(4), 713-729.

Rhoades, L., \& Eisenberger, R. (2002). Perceived organizational support: A review of the literature. Journal of Applied Psychology, 87(4), 698-714. Doi: 10.1037//00219010.87.4.698.

Riketta, M. (2002). Attitudinal organizational commitment and job performance: A metaanalysis. Journal of Organizational Behavior, 23(3). 257-266. Doi:10.1002/job.141.

Robinson, S. L., \& Bennett, R. J. (1995). A typology of deviant workplace behaviors: A multidimensional scaling study. The Academy of Management Journal, 38(2), 555-572.

Rupp, D. E., \& Cropanzano, R. (2002). The mediating effects of social exchange relationship in predicting workplace outcomes from multifoci organizational justice. Organizational Behavior and Human Decision Processes, 89(1), 925-946. 
Schat, A. C. H., \& Kelloway, E. K. (2003). Reducing the adverse consequences of workplace aggression and violence: The buffering effects of organizational support. Journal of Occupational Health Psychology, 8(2), 110-122. Doi:10.1037/10768998.8.2.110.

Schat, A. C. H., Frone, M., \& Kelloway, E. K. (2006). The prevalence of workplace aggression in the U.S. workforce: Findings from a national study. In E. K. Kelloway, J. Barling, \& J. J. Hurrell Jr. (Eds.), Handbook of workplace violence (pp. 579-606). Thousand Oaks, CA: Sage. doi:10.4135/9781412976947.n25.

Schneider, B. (1975). Organizational climates: An essay. Personnel Psychology, 28(4), 447-479.

Schneider, B., \& Reichers, A. E. (1983). On the etiology of climates. Personnel Psychology, 36(1), 19-39.

Schwepker, C. H., Jr. (2001). Ethical climate's relationship to job satisfaction, organizational commitment, and turnover intention in the salesforce. Journal of Business Research, 54(1), 39-52.

Shapiro, S.S. \& Wilk, M.B. (1965). An analysis of variance test for normality (complete samples). Biometrika, 52(3/4). 591-611.

Shockley, K. M., Ipsas, D., Rossi, M. E., \& Levine, E. L. (2012). A meta-analytic investigation of the relationship between state affect, discrete emotions, and job performance. Human Performance, 25(5), 377-411.

DOI:10.1080/08959285.2012.721832.

Shoss, M. K., Eisenberger, R., Restubog, S. L. D., \& Zagenczyk, T. J. (2013). Blaming the organization for abusive supervision: The roles of perceived organizational support and supervisor's organizational embodiment. Journal of Applied Psychology, 98(1), 158168. Doi:10.1037/a0030687.

Simha, A., \& Cullen, J. B. (2012). Ethical climates and their effects on organizational outcomes: Implications from the past and prophecies for the future. The Academy of Management Perspectives, 26(4), 20-34. Doi:10.546S/amp.2011.01S6.

Simon, L.S., Hurst, C., Kelley, K., \& Judge, T. A. (2015). Understanding cycles of abuse: A multimotive approach. Journal of Applied Psychology, 100(6), 1798-1810.

Sobel, M. E., (1982). Asymptotic confidence intervals for indirect effects in structural equation models. Sociological Methodology, 13, 290-312. doi:10.2307/270723.

Society for Human Resource Management (2012, February). Findings from survey on workplace bullying. Retrieved from https://www.shrm.org/hr-today/trends-andforecasting/research-and-surveys/Documents/WorkplaceBullying_Final.pptx. 
Spector, P. E., \& Fox, S. (2002). An emotion-centered model of voluntary work behavior: Some parallels between counterproductive work behavior and organizational citizenship behavior. Human Resource Management Review, 12(2), 269-292.

Spector, P. E., \& Jex, S. M. (1998). Development of four self-report measures of job stressors and strain: interpersonal conflict at work scale, organizational constraints scale, quantitative workload inventory, and physical symptoms inventory. Journal of Occupational Health Psychology, 3(4), 356-367.

Spector, P. E., Coulter, M. L., Stockwell, H. G., \& Matz, M. W. (2007). Perceived violence climate: A new construct and its relationship to workplace physical violence and verbal aggression, and their potential consequences. Work \& Stress, 21(2), 117-130. Doi:10.1080/02678370701410007.

Spector, P. E., Fox, S., \& Van Katwyk, P. T. (1999). The role of negative affectivity in employee reactions to job characteristics: Bias effect or substantive effect? Journal of Occupational and Organizational Psychology, 72(2), 205-218.

Steinert, J. K., (2015). Workplace aggression: A reconceptualization of the construct \& an exploration of strain-based outcomes. ProQuest Dissertations and Theses, 3721568.

Sund, B. \& Lines, O. R., (2017). When leadership leads to loathing: The effect of culturally (in)congruent leadership on employee contempt and voluntary work behaviors. The Journal of Values-Based Leadership, 10(2), 11. Doi:10.22543/0733.102.1191.

Tellegen, A., Watson, D., \& Clark, L. A. (1999). Further support for a hierarchical model of affect: Reply to green and salovey. Psychological Science, 10(4), 307-309.

Tepper, B. J. (2000). Consequences of abusive supervision. Academy of Management Journal, 43(2), 178-190. Doi:10.2307/1556375.

Tepper, B. J. (2007). Abusive supervision in work organizations: Review synthesis, and research agenda. Journal of Management, 33(3), 261-289.

Tepper, B. J., \& Almeda, M. (2012). Negative exchanges with supervisors. In Eby, L, \& Allen, T. (Eds.) Personal Relationships at Work: The Effect of Supervisory, Co-worker, Team, and Customer and Nonwork Exchanges on Employee Attitudes, Behavior, and Well-being (pp. 67-93). New York: Routledge Press.

Tepper, B. J., Carr, J. C., Breaux, D. M., Geider, S., Hu, C., \& Hua, W. (2009). Abusive supervision, intentions to quit, and employee's workplace deviance: A power/dependence analysis. Organizational Behavior and Human Decision Processes, 109(2), 156-167.

Tepper, B. J., Henle, C. A., Lambert, L. S., Giacalone, R. A., \& Duffy, M. K. (2008). Abusive supervision and subordinates' organization deviance. Journal of Applied Psychology, 93(4), 721-732. 
Tepper, B. J., Moss, S. E., \& Duffy, M. K. (2011). Predictors of abusive supervision: Supervisor perceptions of deep-level dissimilarity, relationship conflict, and subordinate performance. The Academy of Management Journal, 54(2), 279-294.

Tepper, B. J., Moss, S. E., Lockhart, D. E., \& Carr, J. C. (2007). Abusive supervision, upward maintenance communication, and subordinates' psychological distress. The Academy of Management Journal, 50(5), 1169-1180.

Tepper, B. J., Simon, L., \& Park, H. M. (2017). Abusive supervision. Annual Review of Organizational Psychology and Organizational Behavior, 4, 123-152.

Tepper, B.J., Simon, L.S., (2015). Employee maintenance: Examining employment relationships from the perspective of managerial leaders. In Buckley, M. R., Wheeler, A.R., \& Halbesleben, J. R. B., (Eds.) Research in Personnel and Human Resources Management (33, pp. 1-50). Emerald Group Publishing Limited.

Tsai, W., Chen, C., \& Liu, H. (2007). Test of a model linking employee positive moods and task performance. Journal of Applied Psychology, 92(6), 1570-1583.

Doi:10.1037/0021-9010.92.6.1570.

U.S. Postal Service Commission (2000, August). Report of the united states postal service commission on a safe and secure workplace. National Center on Addiction and Substance Abuse at Columbia University: New York, NY.

Vardi, Y. (2001). The effects of organizational and ethical climates on misconduct at work. Journal of Business Ethics, 29(4), 325-337.

Victor, B., \& Cullen, J. B. (1988). The organizational bases of ethical work climates. Administrative Science Quarterly, 33(1), 101-125.

Viswesvaran, C. \& Ones, D. S. (2000). Perspectives on models of job performance. International Journal of Selection and Assessment, 8(4), 216-226.

Viswesvaran, C., \& Ones, D. S. (2002). Examining the construct of organizational justice: A meta-analytic evaluation of relations with work attitudes and behaviors. Journal of Business Ethics, 38(3), 193-203.

Walter, F., Lam, C. K., van der Vegt, G. S., Huang, X., \& Miao, Q. (2015). Abusive supervision and subordinate performance: instrumentality considerations in the emergence and consequences of abusive supervision. Journal of Applied Psychology, 100(4), 1056-1072.

Wang, R., \& Jiang, J. (2015). How abusive supervisors influence employees' voice and silence: The effects of interactional justice and organizational attribution. The Journal of Social Psychology, 155(3). 204-220. 
Watkins, Fehr, \& He (in press). Whatever it takes: Leaders' perceptions of abusive supervision instrumentality. The Leadership Quarterly. Doi:

10.1016/j.leaqua.2018.09.002.

Weiss, H. M. (2002). Deconstructing job satisfaction: Separating evaluations, beliefs, and affective experiences. Human Resource Management Review, 12(2), 173-194.

Weiss, H. M., \& Cropanzano, R. (1996). Affective events theory: A theoretical discussion of the structure, causes and consequences of affective experiences at work. In B. M. Staw \& L. L. Cummings (Eds.), Research in Organizational Behavior (pp. 1-74). Greenwich, CT: JAI Press.

Weiss, H. M., Suckow, K., \& Cropanzano, R. (1999). Effects of justice conditions on discrete emotions. Journal of Applied Psychology, 84(5), 786-794.

Welbourne, J. L., \& Sariol, A. M. (2017). When does incivility lead to counterproductive work behavior? Roles of job involvement, task interdependence, and gender. Journal of Occupational Health Psychology, 22(2), 194-206.

Westland, J.C. (2010). Lower bounds on sample size in structural equation modeling. Electronic Commerce Research and Applications, 9(6). 476-487.

Whitman, M. V., Halbesleben, J. R. B., \& Holmes IV, O. (2014). Abusive supervision and feedback avoidance: The mediating role of emotional exhaustion. Journal of Organizational Behavior, 35(1): 38-53.

Williams, L. J., \& Anderson, S. E. (1991). Job satisfaction and organizational commitment as predictors of organizational citizenship and in-role behaviors. Journal of Management, 17(3), 601-617.

Wimbush, J. C, \& Shepard, J. M. (1994). Toward an understanding of ethical climate: Its relationship to ethical behavior and supervisory influence. Journal of Business Ethics, 13(8), 637-647.

Wolf, E. J., Harrington, K. M., Clark, S. L., \& Miller, M. W. (2013). Sample size requirements for structural equation models: An evaluation of power, bias, and solution propriety. Educational and Psychological Measurement, 73(6), 913-934.

Doi:10.1177/0013164413495237.

Wu, T.Y., \& Hu, C. (2009). Abusive supervision and employee emotional exhaustion: Dispositional antecedents and boundaries. Group \& Organization Management, 34(2), 143-169.

Xu, A. J., Loi, R., \& Lam, L. W. (2015). The bad boss takes it all: how abusive supervision and leader-member exchange interact to influence employee silence. The Leadership Quarterly, 26(5), 763-774. 
Xu, E., Huang, X., Lam, C. K., \& Miao, Q. (2012). Abusive supervision and work behaviors: The mediating role of lmx. Journal of Organizational Behavior, 33(4), 531543. Doi:10.1002/job.768.

Yagil, D. (2006). The relationship of abusive and supportive workplace supervision to employee burnout and upward influence tactics. Journal of Emotional Abuse, 6(1). 49-65.

Yagil, D., Ben-Zur, H., \& Tamir, I. (2011). Do employees cope effectively with abusive supervision at work? An exploratory study. International Journal of Stress Management, 18(1), 5-23.

Yu, K., Lin, W., Wang, L., Ma, J., Wei, W., Wang, H., Guo, W., \& Shi, J. (2016). The role of affective commitment and future work self-salience in the abusive supervision-job performance relationship. Journal of Occupational and Organizational Psychology, 89(1), 28-45. Doi:10.1111/joop.12101.

Zellars, K. L., Tepper, B. J., \& Duffy, M. K. (2002). Abusive supervision and subordinates' organizational citizenship behavior. Journal of Applied Psychology, 87(6), $1068-1076$.

Zhang Y., \& Liao Z. (2015). Consequences of abusive supervision: a meta-analytic review. Asia Pacific Journal of Management, 32(4), 959-987.

Zhang, Y., \& Bednall, T. M. (2016). Antecedents of abusive supervision: A meta-analytic review. Journal of Business Ethics, 139(3), 455-471. Doi:10.1007/s10551-015-2657-6.

Zhao, X., Lynch, J. G., \& Chen, Q., (2010). Reconsidering baron and kenny: Myths and truths about mediation analysis. Journal of Consumer Research, 37(2). 197-206.

Zohar, D. (2010). Thirty years of safety climate research: Reflections and future directions. Accident Analysis and Prevention, 42(5), 1517-1522. 
$\underline{\text { Table 1. Meta-analytic relationships between abusive supervision and study variables. }}$

\begin{tabular}{cccccccccc}
\hline & \multicolumn{1}{c}{ Meta-analysis } \\
Study Variable & \multicolumn{7}{c}{ Mackey } & et al. $(2015)$ & \multicolumn{7}{c}{ Zhang \& Liao (2015) } \\
\hline & $\mathrm{n}$ & $\mathrm{k}$ & $\bar{r}$ & $95 \mathrm{CI}$ & $\mathrm{n}$ & $\mathrm{k}$ & $\bar{r}$ & $95 \mathrm{CI}$ \\
Attitudes - Supervisor & & & & & & & & \\
LMX & 2786 & 11 & -.54 & $-.70,-.39$ & & & & \\
IJ & 829 & 5 & -.39 & $-.64,-.15$ & 3916 & 14 & -.51 & $-.60,-.42$ \\
Attitudes - Organization & & & & & & & & \\
POS & 1603 & 7 & -.34 & $-.55,-.25$ & & & & \\
AOC & 2758 & 9 & -.26 & $-.31,-.21$ & 2423 & 10 & -.30 & $-.34,-.25$ \\
Performance & & & & & & & & \\
Task & 4012 & 16 & -.19 & $-.33,-.05$ & 3346 & 14 & -.16 & $-.23,-.09$ \\
OCB & 2842 & 13 & -.24 & $-.31,-.17$ & 1319 & 6 & -.24 & $-.31,-.17$ \\
OCBI & & & & & 2007 & 7 & -.21 & $-.28,-.14$ \\
OCBO & & & & & 1848 & 8 & -.17 & $-.24,-.09$ \\
CWB & 1715 & 7 & .37 & $.11, .72$ & & & & \\
CWBI & 3726 & 13 & .35 & $.23, .47$ & 4470 & 14 & .37 & $.30, .42$ \\
CWBO & 7761 & 22 & .41 & $.24, .57$ & 9917 & 28 & .38 & $.31, .44$ \\
\hline
\end{tabular}

Note. $\mathrm{LMX}=$ Leader-member exchange, $\mathrm{IJ}=$ Interactional Justice, POS=Perceived organizational support, $\mathrm{AOC}=\mathrm{Affective}$ organizational commitment, OCBI $=$ organizational citizenship behaviors directed towards individuals, $\mathrm{OCBO}=$ organizational citizenship behaviors directed towards organization, $\mathrm{CWBI}=$ counterproductive work behaviors directed towards individuals, $\mathrm{CWBO}=$ counterproductive work behaviors directed towards organization. 
Table 2. Corrected correlations between mediators and job performance variables

\begin{tabular}{llllllll}
\hline & \multicolumn{7}{c}{ Job Performance Dimension } \\
Mediator & Task & OCB & OCBI & OCBO & CWB & CWBI & CWBO \\
\hline State PA & $.20^{1}$ & $.32^{2}$ & & & $-.25^{3}$ & & \\
State NA & $-.14^{4}$ & $-.02^{5}$ & & & $.44^{6}$ & & \\
LMX & $.30^{7}$ & $.34^{8}$ & $.38^{9}$ & $.31^{10}$ & $-.24^{11}$ & & \\
IJ $^{\mathrm{a}}$ & $.16^{12}$ & $.43^{13}$ & $.31^{14}$ & $.37^{15}$ & $-.24^{16}$ & $-.25^{17}$ & $-.21^{18}$ \\
POS & $.18^{19}$ & $.22^{20}$ & $.22^{21}$ & $.29^{22}$ & & $-.19^{23}$ & $-.21^{24}$ \\
AOC & $.18^{25}$ & $.23^{26}$ & $.32^{27}$ & $.37^{28}$ & & & \\
\hline
\end{tabular}

Note. A. Some values reflect interpersonal justice and others interactional justice. Across the 47 studies in Colquitt et al.'s (2013) meta-analysis, the corrected correlation between interpersonal and informational justice - the two subdimensions of interactional justice-was .74

$(95 \% \mathrm{CI}=.55, .94)$.

${ }^{1}$ Shockley, Ipsas, Rossi, \& Levine, 2012; $\mathrm{k}=14, \mathrm{n}=1306, \mathrm{SD}=.06$

${ }^{2}$ Shockley, et al., 2012; $\mathrm{k}=24, \mathrm{n}=3411, \mathrm{SD}=.05$

${ }^{3}$ Shockley, et al., 2012; $\mathrm{k}=13, \mathrm{n}=2028, \mathrm{SD}=10$

${ }^{4}$ Shockley, et al., 2012; $\mathrm{k}=18, \mathrm{n}=2134, \mathrm{SD}=.09$

${ }^{5}$ Shockley, et al., 2012; $\mathrm{k}=17, \mathrm{n}=2595, \mathrm{SD}=.04$

${ }^{6}$ Shockley, et al., 2012; $\mathrm{k}=15, \mathrm{n}=2658, \mathrm{SD}=.07$

${ }^{7}$ Martin, Guillaume, Thomas, Lee, Epitropaki, 2016; $\mathrm{k}=146, \mathrm{n}=32670, \mathrm{SD}=.13$

${ }^{8}$ Martin, et al., 2016; $\mathrm{k}=97, \mathrm{n}=23039, \mathrm{SD}=.15$

${ }^{9}$ Ilies, et al., $2007 ; \mathrm{k}=27, \mathrm{n}=5296, \mathrm{SD}=.12$

${ }^{10}$ Ilies, et al., 2007; $\mathrm{k}=21, \mathrm{n}=4119, \mathrm{SD}=.07$

${ }^{11}$ Martin, Guillaume, Thomas, Lee, Epitropaki, 2016; $\mathrm{k}=19, \mathrm{n}=6342, \mathrm{SD}=.18$

${ }^{12}$ Colquitt, Scott, Rodell, Long, Zapata, Conlon \& Wesson, 2013; $\mathrm{k}=11, \mathrm{n}=3542, \mathrm{SD}=.11$

${ }^{13}$ Colquitt et al., 2013; $\mathrm{k}=13, \mathrm{~N}=2533, \mathrm{SD}=.22$

${ }^{14}$ Colquitt et al., 2013; $\mathrm{K}=4, \mathrm{n}=1146, \mathrm{SD}=.10$

${ }^{15}$ Colquitt et al., 2013; $\mathrm{K}=9, \mathrm{n}=1760, \mathrm{SD}=.17$

${ }^{16}$ Colquitt et al., 2013; $\mathrm{k}=10, \mathrm{n}=2043, \mathrm{SD}=.10$

${ }^{17}$ Berry, Ones \& Sackett, 2007; $\mathrm{k}=6, \mathrm{n}=1208, \mathrm{SD}=13$

${ }^{18}$ Berry, et al., 2007, $\mathrm{k}=6, \mathrm{n}=1190, \mathrm{SD}=.07$

${ }^{19}$ Rhoades \& Ei senberger, 2002; $\mathrm{k}=12, \mathrm{n}=2873, \mathrm{SD}=.13$

${ }^{20}$ Rhoades \& Eisenberger, 2002; $\mathrm{k}=13, \mathrm{n}=3760, \mathrm{SD}=.05$

${ }^{21}$ Rhoades \& Ei senberger, 2002; $\mathrm{k}=9, \mathrm{n}-1924, \mathrm{SD}=.12$

${ }^{22}$ Rhoades \& Eisenberger, 2002; $\mathrm{k}=7, \mathrm{n}=2010, \mathrm{SD}=.04$

${ }^{23}$ Kurtessis, Eisenberger, Ford, Buffardi, Stewart, \& Adis, 2016; k=7, n=1860, SD=.11

${ }^{24}$ Kurtessis, et al., 2016; $\mathrm{k}=14, \mathrm{n}=4315, \mathrm{SD}=.10$

${ }^{25}$ Riketta, 2002; $\mathrm{k}=87, \mathrm{n}=20973 ; \mathrm{SD}=.01$

${ }^{26} \mathrm{Ng} \&$ Feldman, $2011 ; \mathrm{k}=64, \mathrm{n}=17509, \mathrm{SD}=.08$ [externally rated $\mathrm{OCB}$ ]

${ }^{27}$ Cetin, et al., 2015; $\mathrm{k}=27, \mathrm{~N}=8942, \mathrm{SD}=0.17$

${ }^{28}$ Cetin, et al., 2015; $\mathrm{k}=26, \mathrm{~N}=8931, \mathrm{SD}=0.20$ 
Table 3a. Data cleaning efforts for focal employee participants

\begin{tabular}{ccc}
\hline Criterion & No. of Cases & Percent \\
\hline PERSONS WHO AGREED TO PARTICIPATE & \\
Completed eligibility & 6905 & 80.69 \\
Did not complete eligibility & 1652 & 19.31 \\
Agreed to participate & 8557 & 100.00 \\
\hline PARTICIPANTS WHO COMPLETED ELIGIBILITY SECTION \\
Met eligibility criteria & 3292 & 47.68 \\
Did not meet eligibility criteria & 3613 & 52.32 \\
CASES REMOVED due to: & 1660 & \\
Time < 400 secs & 1 & \\
Age $<18$ & 522 & \\
Employment Status & 384 & \\
No boss & 7 & \\
Work outside US & 546 & \\
Weekly work hours $<$ 20 & 493 & \\
Org Tenure $<$ one year & 6905 & 100.00 \\
\hline PARTICIPANTS WHO MET ELIGIBILITY CRITERIA \\
Provided usable data & 2738 & 83.17 \\
Did not provide usable data & 554 & 16.83 \\
TOTAL CASES REMOVED due to: & & \\
ACQ fails $>1$ & 11 & \\
Missing Data & 543 & 100.00 \\
\hline
\end{tabular}


Table 3b. Data cleaning efforts for coworker participants

\begin{tabular}{ccc}
\hline Criterion & No. of Cases & Percent \\
\hline \multicolumn{4}{c}{ PERSONS WHO AGREED TO PARTICIPATE } \\
Provided valid InviteCode & 572 & 71.95 \\
Did not provide valid InviteCode & 223 & 28.05 \\
Agreed to participate & 795 & 100.00 \\
\hline \multicolumn{3}{c}{ PARTICIPANTS WHO PROVIDED VALID INVITE CODE } \\
Completed eligibility & 511 & 89.34 \\
Did not complete eligibility & 61 & 10.66 \\
Provided valid InviteCode & 572 & 100.00 \\
\hline PARTICIPANTS WHO COMPLETED ELIGIBILITY SECTION \\
Met eligibility criteria & 300 & 58.71 \\
Did not meet eligibility criteria & 211 & 41.29 \\
\multicolumn{4}{c}{ CASES REMOVED due to: } & 29 & \\
Time $<240$ secs & 0 & \\
Age $<$ 18 & 4 & \\
Employment Status & 110 & \\
Peer relationship & 13 & \\
Coworker interactions & 24 & \\
Job Knowledge & 1 & \\
Work outside US & 10 & \\
Weekly work hours $<20$ & 20 & \\
Org Tenure $<$ one year & 511 & 100.00 \\
\hline \multicolumn{4}{c}{ PARTICIPANTS WHO MET ELIGIBILITY CRITERIA } \\
Provided usable data & 281 & 93.67 \\
Did not provide usable data & 19 & 6.33 \\
TOTAL CASES REMOVED due to: & & \\
ACQ fails $>0$ & 17 & \\
Missing Data & 0 & \\
More than one coworker & 2 & \\
Met eligibility criteria & 300 & 100.00 \\
\hline
\end{tabular}


Table 4. Variable distributions across matched and unmatched groups (continuous variables)

\begin{tabular}{|c|c|c|c|c|c|c|}
\hline \multirow[b]{2}{*}{ Characteristic } & \multicolumn{2}{|c|}{ Full Dataset ${ }^{\mathrm{A}}$} & \multicolumn{2}{|c|}{$\begin{array}{l}\text { Matched- } \\
\text { Only }^{\mathrm{B}}\end{array}$} & \multirow{2}{*}{$\begin{array}{c}\text { Sig. (2- } \\
\text { tailed) } \\
\text { Difference } \\
\text { in Means }\end{array}$} & \multirow[t]{2}{*}{$\begin{array}{c}\text { Effect Size } \\
\text { (Hedge's } \\
g \text { ) }\end{array}$} \\
\hline & Mean & $\mathrm{SD}$ & Mean & SD & & \\
\hline Age (Years) & 33.83 & 9.35 & 33.56 & 8.99 & .64 & .03 \\
\hline Org. Tenure (Years) & 5.88 & 5.36 & 5.98 & 5.34 & .67 & .02 \\
\hline Average Hours Worked & 40.29 & 8.15 & 40.09 & 7.27 & .76 & .02 \\
\hline Employee PEC & 3.42 & .47 & 3.49 & .52 & .03 & .15 \\
\hline Abusive Supervision & 1.96 & 1.14 & 1.49 & .78 & .00 & .42 \\
\hline Perceived Org. Support & 3.47 & .78 & 3.51 & .88 & .49 & .05 \\
\hline Affective Org. & 3.26 & .79 & 3.29 & .87 & .58 & .04 \\
\hline Commitment & & & & & & \\
\hline $\begin{array}{l}\text { Leader-Member } \\
\text { Exchange }\end{array}$ & 3.50 & .85 & 3.58 & .87 & .15 & .09 \\
\hline Interactional Justice & 3.81 & .82 & 3.85 & .88 & .43 & .05 \\
\hline Positive Affect & 3.38 & .90 & 3.15 & .91 & .00 & .26 \\
\hline Negative Affect & 2.35 & 1.02 & 1.98 & .86 & .00 & .37 \\
\hline
\end{tabular}

Note. A. $N=281 ;$ B. $N=2738 ;$ PEC $=$ Perceived ethical climate. Median age $=31,32$, and 33 for Full dataset, Matched-Only, and US Labor Force (2018), respectively. 
Table 5. Demographic characteristics across study datasets and US labor force

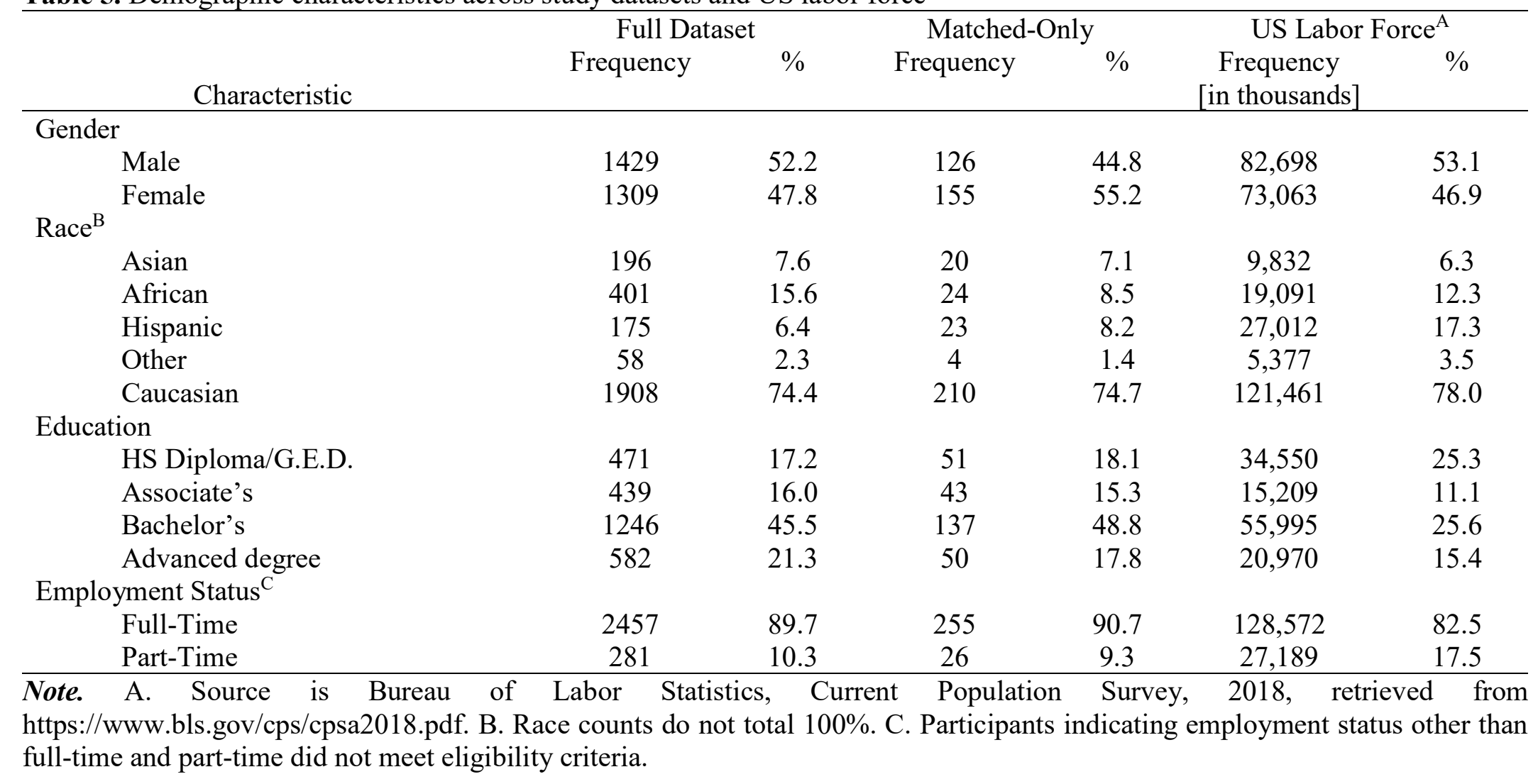


Table 6a. Pearson zero-order correlations and internal reliability estimates (full dataset; $\mathrm{N}=2738$ )

\begin{tabular}{|c|c|c|c|c|c|c|c|c|}
\hline Variable & 1 & 2 & 3 & 4 & 5 & 6 & 7 & 8 \\
\hline 1. AS (Self-reported) & $(.98)$ & & & & & & & \\
\hline 2. Employee PEC & $-.27^{*}$ & $(.80)$ & & & & & & \\
\hline 3. POS & $-.26^{*}$ & $.70^{*}$ & $(.86)$ & & & & & \\
\hline 4. $\mathrm{AOC}$ & $-.28^{*}$ & $.58^{*}$ & $.70^{*}$ & $(.82)$ & & & & \\
\hline 5. LMX & $-.35^{*}$ & $.52^{*}$ & $.60^{*}$ & $.52^{*}$ & $(.90)$ & & & \\
\hline 6. IJ & $-.27^{*}$ & $.63^{*}$ & $.67^{*}$ & $.51^{*}$ & $.74^{*}$ & $(.90)$ & & \\
\hline 7. PA (State) & $.04^{*}$ & $.55^{*}$ & $.63^{*}$ & $.59^{*}$ & $.51^{*}$ & $.58^{*}$ & $(.93)$ & \\
\hline 8. NA (State) & $.67^{*}$ & $-.38^{*}$ & $-.42^{*}$ & $-.47^{*}$ & $-.37^{*}$ & $-.32^{*}$ & $-.29^{*}$ & $(.96)$ \\
\hline
\end{tabular}


Table 6b. Pearson zero-order correlations and internal reliability estimates (matched-only dataset; N=281)

\begin{tabular}{|c|c|c|c|c|c|c|c|c|c|c|c|c|c|c|c|}
\hline Variable & 1 & 2 & 3 & 4 & 5 & 6 & 7 & 8 & 9 & 10 & 11 & 12 & 13 & 14 & 15 \\
\hline 1 AS (Self) & $(.97)$ & & & & & & & & & & & & & & \\
\hline $2 \mathrm{AS}$ (Other) & $.86^{*}$ & $(.97)$ & & & & & & & & & & & & & \\
\hline 3 E-PEC & $-.40 *$ & $-.39 *$ & $(.85)$ & & & & & & & & & & & & \\
\hline 4 C-PEC & $-.37 *$ & $-.40 *$ & $.77^{*}$ & $(.85)$ & & & & & & & & & & & \\
\hline $5 \mathrm{POS}$ & $-.36^{*}$ & $-.35 *$ & $.76^{*}$ & $.62^{*}$ & $(.91)$ & & & & & & & & & & \\
\hline $6 \mathrm{AOC}$ & $-.25 *$ & $-.26^{*}$ & $.63 *$ & $.50 *$ & $.72 *$ & $(.88)$ & & & & & & & & & \\
\hline 7 LMX & $-.48 *$ & $-.44 *$ & $.63^{*}$ & $.52 *$ & $.68 *$ & $.60^{*}$ & $(.92)$ & & & & & & & & \\
\hline $8 \mathrm{IJ}$ & $-.51 *$ & $-.46^{*}$ & $.66^{*}$ & $.52 *$ & $.71^{*}$ & $.56^{*}$ & $.81^{*}$ & $(.93)$ & & & & & & & \\
\hline 9 PA (State) & $-.13 \dagger$ & $-.17 *$ & $.60 *$ & $.49^{*}$ & $.67^{*}$ & $.64 *$ & $.60 *$ & $.60^{*}$ & (.94) & & & & & & \\
\hline 10 NA (State) & $.55^{*}$ & $.58 *$ & $-.47 *$ & $-.43 *$ & $-.48^{*}$ & $-.39 *$ & $-.40 *$ & $-.41 *$ & $-.44 *$ & (.93) & & & & & \\
\hline 11 TASK & $-.42 *$ & $-.34 *$ & $.23^{*}$ & $.26^{*}$ & $.13 \dagger$ & .02 & $.25^{*}$ & $.25^{*}$ & .06 & $-.21^{*}$ & $(.91)$ & & & & \\
\hline $12 \mathrm{OCBO}$ & $-.16 \dagger$ & -.10 & $.23 *$ & $.24 *$ & $.24 *$ & $.23 *$ & $.33 *$ & $.34 *$ & $.33 *$ & -.06 & $.41 *$ & $(.84)$ & & & \\
\hline 13 OCBI & -.10 & -.08 & $.23 *$ & $.29 *$ & $.21 *$ & $.18^{*}$ & $.27 *$ & $.22 *$ & $.24 *$ & -.01 & $.45^{*}$ & $.69^{*}$ & $(.84)$ & & \\
\hline $14 \mathrm{CWBO}$ & $.59^{*}$ & $.55^{*}$ & $-.17 *$ & $-.21 *$ & $-.14 \dagger$ & -.07 & -.12 & -.10 & .09 & $.36^{*}$ & $-.51 *$ & -.11 & -.12 & $(.95)$ & \\
\hline $15 \mathrm{CWBI}$ & $.58 *$ & $.56^{*}$ & $-.14 *$ & $-.19 *$ & $-.16^{*}$ & -.06 & $-.12 \dagger$ & $-.13 \dagger$ & .11 & $.36^{*}$ & $-.47 *$ & $-.14 \dagger$ & $-.15 \dagger$ & $.89 *$ & $(.95)$ \\
\hline
\end{tabular}

Note. Internal reliability estimates (Cronbach alphas) appear, in parentheses, along diagonal. $\uparrow=p<.05, * p<.01 ;$ AS $=$ Abusive supervision; E-PEC = Employee perceived ethical climate; C-PEC = coworker perceived ethical climate; AOC = Affective Organizational Commitment; POS = Perceived Organizational Support; LMX = Leader-Member Exchange; IJ= Interactional Justice; PA = Positive Affect; NA = Negative Affect; TASK=Task Performance; OCBO=Organizational Citizenship BehaviorsOrganizational; OCBI=Organizational Citizenship Behaviors-Interpersonal; CWBO=Counterproductive Work BehaviorsOrganizational; $\mathrm{CWBI}=$ Counterproductive Work Behaviors-Interpersonal. 
Table 7a. Spearman's rho correlations (full dataset; N=2738)

\begin{tabular}{|c|c|c|c|c|c|c|c|c|}
\hline Variable & 1 & 2 & 3 & 4 & 5 & 6 & 7 & 8 \\
\hline 1. AS (Self-reported) & 1.00 & & & & & & & \\
\hline 2. Employee PEC & $-.35^{*}$ & 1.00 & & & & & & \\
\hline 3. POS & $-.37^{*}$ & $.68^{*}$ & 1.00 & & & & & \\
\hline 4. $\mathrm{AOC}$ & $-.35^{*}$ & $.55^{*}$ & $.66^{*}$ & 1.00 & & & & \\
\hline 5. LMX & $-.45^{*}$ & $.52^{*}$ & $.59^{*}$ & $.49^{*}$ & 1.00 & & & \\
\hline 6. IJ & $-.37^{*}$ & $.62^{*}$ & $.63^{*}$ & $.46^{*}$ & $.70^{*}$ & 1.00 & & \\
\hline 7. PA (State) & $-.04^{\dagger}$ & $.51^{*}$ & $.57^{*}$ & $.52^{*}$ & $.48^{*}$ & $.55^{*}$ & 1.00 & \\
\hline 8. NA (State) & $-.63^{*}$ & $-.40^{*}$ & $-.46^{*}$ & $-.50^{*}$ & $-.40^{*}$ & $-.34^{*}$ & $-.31^{*}$ & 1.00 \\
\hline
\end{tabular}

Note. ${ }^{\dagger}=\mathrm{p}<.05,{ }^{*} \mathrm{p}<.01 ; \mathrm{AS}=$ Abusive Supervision; PEC= Perceived Ethical Climate PA= Positive Affect; NA = Negative Affect; $\mathrm{AOC}=$ Affective Organizational Commitment; $\mathrm{POS}=$ Perceived Organizational Support; LMX= Leader-Member Exchange; IJ= Interactional Justice. 
Table 7b. Spearman's rho correlations (matched-only group; $\mathrm{N}=281$ )

\begin{tabular}{|c|c|c|c|c|c|c|c|c|c|c|c|c|c|c|c|}
\hline Variable & 1 & 2 & 3 & 4 & 5 & 6 & 7 & 8 & 9 & 10 & 11 & 12 & 13 & 14 & 15 \\
\hline 1 AS (Self) & 1.00 & & & & & & & & & & & & & & \\
\hline 2 AS (Other) & $.83^{*}$ & 1.00 & & & & & & & & & & & & & \\
\hline 3 E-PEC & $-.44^{*}$ & $-.39^{*}$ & 1.00 & & & & & & & & & & & & \\
\hline 4 C-PEC & -.07 & $-.13^{\dagger}$ & $.75^{*}$ & 1.00 & & & & & & & & & & & \\
\hline 5 POS & $-.51^{*}$ & $-.46^{*}$ & $.74^{*}$ & $.33^{*}$ & 1.00 & & & & & & & & & & \\
\hline $6 \mathrm{AOC}$ & $-.33^{*}$ & $-.32^{*}$ & $.62^{*}$ & $.18^{*}$ & $.70^{*}$ & 1.00 & & & & & & & & & \\
\hline 7 LMX & $-.56^{*}$ & $-.50^{*}$ & $.63^{*}$ & $.24^{*}$ & $.70^{*}$ & $.59^{*}$ & 1.00 & & & & & & & & \\
\hline $8 \mathrm{IJ}$ & $-.61^{*}$ & $-.51^{*}$ & $.62^{*}$ & $.27^{*}$ & $.72^{*}$ & $.55^{*}$ & $.79^{*}$ & 1.00 & & & & & & & \\
\hline 9 PA (State) & $-.22^{*}$ & $-.25^{*}$ & $.56^{*}$ & $.35^{*}$ & $.64^{*}$ & $.64^{*}$ & $.58^{*}$ & $.55^{*}$ & 1.00 & & & & & & \\
\hline 10 NA (State) & $.54^{*}$ & $.56^{*}$ & $-.44^{*}$ & $-.14^{\dagger}$ & $-.49^{*}$ & $-.43^{*}$ & $-.40^{*}$ & $-.42^{*}$ & $-.46^{*}$ & 1.00 & & & & & \\
\hline 11 TASK & $-.37^{*}$ & $-.33^{*}$ & $.26^{*}$ & .10 & $.18^{*}$ & .04 & $.26^{*}$ & $.28^{*}$ & .05 & $-.19^{*}$ & 1.00 & & & & \\
\hline $12 \mathrm{OCBO}$ & $-.20^{*}$ & $-.15^{*}$ & $.24^{*}$ & $.30^{*}$ & $.26^{*}$ & $.22^{*}$ & $.33^{*}$ & $.32^{*}$ & $.30^{*}$ & -.09 & $.35^{*}$ & 1.00 & & & \\
\hline 13 OCBI & $-.15^{\dagger}$ & $-.14^{\dagger}$ & $.28^{*}$ & $.22^{*}$ & $.25^{*}$ & $.19^{*}$ & $.27^{*}$ & $.24^{*}$ & $.22^{*}$ & -.07 & $.40^{*}$ & $.66^{*}$ & 1.00 & & \\
\hline $14 \mathrm{CWBO}$ & $.45^{*}$ & $.43^{*}$ & $-.27^{*}$ & -.05 & $-.22^{*}$ & -.11 & $-.20^{*}$ & $-.20^{*}$ & -.03 & $.38^{*}$ & $-.55^{*}$ & $-.21^{*}$ & $-.21^{*}$ & 1.00 & \\
\hline $15 \mathrm{CWBI}$ & $.49^{*}$ & $.52^{*}$ & $-.25^{*}$ & -.05 & $-.24^{*}$ & $-.13^{\dagger}$ & $-.20^{*}$ & $-.25^{*}$ & -.00 & $.34^{*}$ & $-.47^{*}$ & $-.22^{*}$ & $-.27^{*}$ & $.65^{*}$ & 1.00 \\
\hline
\end{tabular}

Note $^{\dagger}=\mathrm{p}<.05,{ }^{*} \mathrm{p}<.01 ; \mathrm{AS}=$ Abusive supervision; E-PEC $=$ Employee perceived ethical climate; C-PEC = coworker perceived ethical climate; $\mathrm{AOC}=$ Affective Organizational Commitment; POS = Perceived Organizational Support; LMX $=$ Leader-Member Exchange; IJ= Interactional Justice; $\mathrm{PA}=$ Positive Affect; NA = Negative Affect; TASK=Task Performance; OCBO=Organizational Citizenship Behaviors-Organizational; OCBI=Organizational Citizenship Behaviors-Interpersonal; $\mathrm{CWBO}=\mathrm{Counterproductive}$ Work Behaviors-Organizational; CWBI=Counterproductive Work Behaviors-Interpersonal. 
Table 8a. Univariate normality tests for all study variables (full dataset)

\begin{tabular}{|c|c|c|c|}
\hline Variable Name & S-W & Df. & Sig. \\
\hline Mean of Abusive Supervision (AS) Items & .796 & 2738 & 0.0000 \\
\hline AS 1 & .705 & 2738 & 0.0000 \\
\hline AS 2 & 659 & 2738 & 0.0000 \\
\hline AS 3 & .727 & 2738 & 0.0000 \\
\hline AS 4 & .697 & 2738 & 0.0000 \\
\hline AS 5 & .701 & 2738 & 0.0000 \\
\hline AS 6 & .752 & 2738 & 0.0000 \\
\hline AS 7 & .793 & 2738 & 0.0000 \\
\hline AS 8 & .705 & 2738 & 0.0000 \\
\hline AS 9 & .785 & 2738 & 0.0000 \\
\hline AS 10 & .759 & 2738 & 0.0000 \\
\hline AS 11 & .715 & 2738 & 0.0000 \\
\hline AS 12 & .746 & 2738 & 0.0000 \\
\hline AS 13 & .672 & 2738 & 0.0000 \\
\hline AS 14 & .634 & 2738 & 0.0000 \\
\hline AS 15 & .726 & 2738 & 0.0000 \\
\hline Mean of Perceived Ethical Climate (PEC) Items & .980 & 2738 & 0.0000 \\
\hline PEC 1 & .856 & 2738 & 0.0000 \\
\hline PEC 2 & .880 & 2738 & 0.0000 \\
\hline PEC 3 & .907 & 2738 & 0.0000 \\
\hline PEC 4 & .871 & 2738 & 0.0000 \\
\hline PEC 5 & .826 & 2738 & 0.0000 \\
\hline PEC 6 & .843 & 2738 & 0.0000 \\
\hline PEC 7 & .829 & 2738 & 0.0000 \\
\hline PEC 8 & .870 & 2738 & 0.0000 \\
\hline PEC 9 & .824 & 2738 & 0.0000 \\
\hline PEC 10 & .826 & 2738 & 0.0000 \\
\hline PEC 11 & .892 & 2738 & 0.0000 \\
\hline PEC 12 & .885 & 2738 & 0.0000 \\
\hline PEC 13 & .907 & 2738 & 0.0000 \\
\hline PEC 14 & .910 & 2738 & 0.0000 \\
\hline PEC 15 & .913 & 2738 & 0.0000 \\
\hline PEC 16 & .897 & 2738 & 0.0000 \\
\hline PEC 17 & .903 & 2738 & 0.0000 \\
\hline PEC 18 & .907 & 2738 & 0.0000 \\
\hline PEC 19 & .913 & 2738 & 0.0000 \\
\hline PEC 20 & .910 & 2738 & 0.0000 \\
\hline PEC 21 & .915 & 2738 & 0.0000 \\
\hline PEC 22 & .913 & 2738 & 0.0000 \\
\hline Mean of Positive Affective States (PA) Items & .980 & 2738 & 0.0000 \\
\hline PA 1 & .896 & 2738 & 0.0000 \\
\hline PA 2 & .905 & 2738 & 0.0000 \\
\hline
\end{tabular}




\begin{tabular}{|c|c|c|c|}
\hline PA 3 & .894 & 2738 & 0.0000 \\
\hline PA 4 & .916 & 2738 & 0.0000 \\
\hline PA 5 & .907 & 2738 & 0.0000 \\
\hline PA 6 & .901 & 2738 & 0.0000 \\
\hline PA 7 & .910 & 2738 & 0.0000 \\
\hline PA 8 & .901 & 2738 & 0.0000 \\
\hline PA 9 & .911 & 2738 & 0.0000 \\
\hline PA 10 & .890 & 2738 & 0.0000 \\
\hline Mean of Negative Affective States (NA) Items & .939 & 2738 & 0.0000 \\
\hline NA 1 & .876 & 2738 & 0.0000 \\
\hline NA 2 & .901 & 2738 & 0.0000 \\
\hline NA 3 & .883 & 2738 & 0.0000 \\
\hline NA 4 & .826 & 2738 & 0.0000 \\
\hline NA 5 & .857 & 2738 & 0.0000 \\
\hline NA 6 & .807 & 2738 & 0.0000 \\
\hline NA 7 & .896 & 2738 & 0.0000 \\
\hline NA 8 & .754 & 2738 & 0.0000 \\
\hline NA 9 & .803 & 2738 & 0.0000 \\
\hline NA 10 & .828 & 2738 & 0.0000 \\
\hline Mean of Perceived Organizational Support (POS) Items & .968 & 2738 & 0.0000 \\
\hline POS 1 & .844 & 2738 & 0.0000 \\
\hline POS 2 & .872 & 2738 & 0.0000 \\
\hline POS 3 & .877 & 2738 & 0.0000 \\
\hline POS 4 & .852 & 2738 & 0.0000 \\
\hline POS 5 & .849 & 2738 & 0.0000 \\
\hline POS 6 & .906 & 2738 & 0.0000 \\
\hline POS 7 & .904 & 2738 & 0.0000 \\
\hline POS 8 & .886 & 2738 & 0.0000 \\
\hline Mean of Affective Organizational Commitment (AOC) Items & .985 & 2738 & 0.0000 \\
\hline AOC 1 & .869 & 2738 & 0.0000 \\
\hline AOC 2 & .882 & 2738 & 0.0000 \\
\hline AOC 3 & .897 & 2738 & 0.0000 \\
\hline $\mathrm{AOC} 4$ & .897 & 2738 & 0.0000 \\
\hline AOC 5 & .893 & 2738 & 0.0000 \\
\hline AOC 6 & .896 & 2738 & 0.0000 \\
\hline AOC 7 & .888 & 2738 & 0.0000 \\
\hline $\mathrm{AOC} 8$ & .898 & 2738 & 0.0000 \\
\hline Mean of Leader-Member Exchange (LMX) Items & .981 & 2738 & 0.0000 \\
\hline LMX 1 & .887 & 2738 & 0.0000 \\
\hline LMX 2 & .891 & 2738 & 0.0000 \\
\hline LMX 3 & .890 & 2738 & 0.0000 \\
\hline LMX 4 & .899 & 2738 & 0.0000 \\
\hline LMX 5 & .917 & 2738 & 0.0000 \\
\hline LMX 6 & .867 & 2738 & 0.0000 \\
\hline
\end{tabular}




\begin{tabular}{llll}
\hline LMX 7 & .875 & 2738 & 0.0000 \\
Mean of Interactional Justice (IJ) Items & .927 & 2738 & 0.0000 \\
IJ 1 & .794 & 2738 & 0.0000 \\
IJ 2 & .876 & 2738 & 0.0000 \\
IJ 3 & .844 & 2738 & 0.0000 \\
IJ 4 & .838 & 2738 & 0.0000 \\
IJ 5 & .835 & 2738 & 0.0000 \\
IJ 6 & .832 & 2738 & 0.0000 \\
\hline
\end{tabular}

Note. $\mathrm{S}-\mathrm{W}=$ Shapiro-Wilk Statistic $(\mathrm{W}) ; \mathrm{Df}=$ Degrees of freedom; Sig. = p-value.; ${ }^{*} \mathrm{p}>$.00053 (Bonferroni-corrected p-value) 
Table 8b. Univariate normality tests for all study variables (matched-only dataset)

\begin{tabular}{|c|c|c|c|}
\hline Variable Name & S-W & Df. & Sig. \\
\hline Mean of Abusive Supervision (AS) Items & .686 & 281 & 0.0000 \\
\hline AS 1 & .574 & 276 & 0.0000 \\
\hline AS 2 & .454 & 276 & 0.0000 \\
\hline AS 3 & .557 & 276 & 0.0000 \\
\hline AS 4 & .511 & 276 & 0.0000 \\
\hline AS 5 & .545 & 276 & 0.0000 \\
\hline AS 6 & .648 & 276 & 0.0000 \\
\hline AS 7 & .691 & 276 & 0.0000 \\
\hline AS 8 & .504 & 276 & 0.0000 \\
\hline AS 9 & .697 & 276 & 0.0000 \\
\hline AS 10 & .610 & 276 & 0.0000 \\
\hline AS 11 & .551 & 276 & 0.0000 \\
\hline AS 12 & .597 & 276 & 0.0000 \\
\hline AS 13 & .460 & 276 & 0.0000 \\
\hline AS 14 & .417 & 276 & 0.0000 \\
\hline AS 15 & .618 & 276 & 0.0000 \\
\hline Mean of Perceived Ethical Climate (PEC) Items & .976 & 281 & 0.0001 \\
\hline PEC 1 & .856 & 276 & 0.0000 \\
\hline PEC 2 & .867 & 276 & 0.0000 \\
\hline PEC 3 & .900 & 276 & 0.0000 \\
\hline PEC 4 & .865 & 276 & 0.0000 \\
\hline PEC 5 & .805 & 276 & 0.0000 \\
\hline PEC 6 & .820 & 276 & 0.0000 \\
\hline PEC 7 & .800 & 276 & 0.0000 \\
\hline PEC 8 & .862 & 276 & 0.0000 \\
\hline PEC 9 & .781 & 276 & 0.0000 \\
\hline PEC 10 & .784 & 276 & 0.0000 \\
\hline PEC 11 & .893 & 276 & 0.0000 \\
\hline PEC 12 & .882 & 276 & 0.0000 \\
\hline PEC 13 & .912 & 276 & 0.0000 \\
\hline PEC 14 & .912 & 276 & 0.0000 \\
\hline PEC 15 & .911 & 276 & 0.0000 \\
\hline PEC 16 & .879 & 276 & 0.0000 \\
\hline PEC 17 & .901 & 276 & 0.0000 \\
\hline PEC 18 & .909 & 276 & 0.0000 \\
\hline PEC 19 & .893 & 276 & 0.0000 \\
\hline PEC 20 & .892 & 276 & 0.0000 \\
\hline PEC 21 & .903 & 276 & 0.0000 \\
\hline PEC 22 & .912 & 276 & 0.0000 \\
\hline Mean of Positive Affective States (PA) Items & $.981^{*}$ & 281 & 0.0008 \\
\hline PA 1 & .889 & 276 & 0.0000 \\
\hline PA 2 & .904 & 276 & 0.0000 \\
\hline PA 3 & .886 & 276 & 0.0000 \\
\hline PA 4 & .902 & 276 & 0.0000 \\
\hline PA 5 & .914 & 276 & 0.0000 \\
\hline PA 6 & .897 & 276 & 0.0000 \\
\hline
\end{tabular}




\begin{tabular}{|c|c|c|c|}
\hline PA 7 & .907 & 276 & 0.0000 \\
\hline PA 8 & .903 & 276 & 0.0000 \\
\hline PA 9 & .912 & 276 & 0.0000 \\
\hline PA 10 & .892 & 276 & 0.0000 \\
\hline Mean of Negative Affective States (NA) Items & .906 & 281 & 0.0000 \\
\hline NA 1 & .842 & 276 & 0.0000 \\
\hline NA 2 & .875 & 276 & 0.0000 \\
\hline NA 3 & .856 & 276 & 0.0000 \\
\hline NA 4 & .733 & 276 & 0.0000 \\
\hline NA 5 & .806 & 276 & 0.0000 \\
\hline NA 6 & .697 & 276 & 0.0000 \\
\hline NA 7 & .878 & 276 & 0.0000 \\
\hline NA 8 & .612 & 276 & 0.0000 \\
\hline NA 9 & .684 & 276 & 0.0000 \\
\hline NA 10 & .712 & 276 & 0.0000 \\
\hline Mean of Perceived Organizational Support (POS) Items & .971 & 281 & 0.0000 \\
\hline POS 1 & .849 & 276 & 0.0000 \\
\hline POS 2 & .877 & 276 & 0.0000 \\
\hline POS 3 & .887 & 276 & 0.0000 \\
\hline POS 4 & .834 & 276 & 0.0000 \\
\hline POS 5 & .839 & 276 & 0.0000 \\
\hline POS 6 & .907 & 276 & 0.0000 \\
\hline POS 7 & .887 & 276 & 0.0000 \\
\hline POS 8 & .881 & 276 & 0.0000 \\
\hline Mean of Affective Organizational Commitment (AOC) Items & .978 & 281 & 0.0003 \\
\hline AOC 1 & .876 & 276 & 0.0000 \\
\hline AOC 2 & .875 & 276 & 0.0000 \\
\hline AOC 3 & .894 & 276 & 0.0000 \\
\hline AOC 4 & .886 & 276 & 0.0000 \\
\hline AOC 5 & .883 & 276 & 0.0000 \\
\hline AOC 6 & .885 & 276 & 0.0000 \\
\hline AOC 7 & .883 & 276 & 0.0000 \\
\hline AOC 8 & .886 & 276 & 0.0000 \\
\hline Mean of Leader-Member Exchange (LMX) Items & .974 & 281 & 0.0000 \\
\hline LMX 1 & .863 & 276 & 0.0000 \\
\hline LMX 2 & .873 & 276 & 0.0000 \\
\hline LMX 3 & .868 & 276 & 0.0000 \\
\hline LMX 4 & .893 & 276 & 0.0000 \\
\hline LMX 5 & .915 & 276 & 0.0000 \\
\hline LMX 6 & .867 & 276 & 0.0000 \\
\hline LMX 7 & .879 & 276 & 0.0000 \\
\hline Mean of Interactional Justice (IJ) Items & .919 & 281 & 0.0000 \\
\hline IJ 1 & .793 & 276 & 0.0000 \\
\hline IJ 2 & .872 & 276 & 0.0000 \\
\hline IJ 3 & .819 & 276 & 0.0000 \\
\hline IJ 4 & .817 & 276 & 0.0000 \\
\hline IJ 5 & .819 & 276 & 0.0000 \\
\hline
\end{tabular}




\begin{tabular}{|c|c|c|c|}
\hline IJ 6 & .825 & 276 & 0.0000 \\
\hline Mean of Task Performance (TASK) Items & .793 & 281 & 0.0000 \\
\hline TASK 1 & .711 & 276 & 0.0000 \\
\hline TASK 2 & .706 & 276 & 0.0000 \\
\hline TASK 3 & 677 & 276 & 0.0000 \\
\hline TASK 4 & 690 & 276 & 0.0000 \\
\hline Mean of Organizational Citizenship Behavior (Org.; OCBO) Items & .973 & 281 & 0.0000 \\
\hline OCBO 1 & .845 & 276 & 0.0000 \\
\hline OCBO 2 & .857 & 276 & 0.0000 \\
\hline OCBO 3 & .869 & 276 & 0.0000 \\
\hline OCBO 4 & .898 & 272 & 0.0000 \\
\hline OCBO 5 & .880 & 276 & 0.0000 \\
\hline OCBO 6 & .903 & 276 & 0.0000 \\
\hline Mean of Organizational Citizenship Behavior (Ind.; OCBI) Items & .973 & 281 & 0.0000 \\
\hline OCBI 1 & .827 & 276 & 0.0000 \\
\hline OCBI 2 & .841 & 276 & 0.0000 \\
\hline OCBI 3 & .902 & 276 & 0.0000 \\
\hline OCBI 4 & .882 & 276 & 0.0000 \\
\hline OCBI 5 & .863 & 276 & 0.0000 \\
\hline OCBI 6 & .876 & 276 & 0.0000 \\
\hline Mean of Counterproductive Work Behaviors (Org.; CWBO) Items & .683 & 281 & 0.0000 \\
\hline CWBO 1 & .611 & 276 & 0.0000 \\
\hline CWBO 2 & .730 & 276 & 0.0000 \\
\hline CWBO 3 & .696 & 276 & 0.0000 \\
\hline CWBO 4 & .615 & 276 & 0.0000 \\
\hline CWBO 5 & .529 & 276 & 0.0000 \\
\hline CWBO 6 & .597 & 276 & 0.0000 \\
\hline CWBO 7 & .551 & 276 & 0.0000 \\
\hline CWBO 8 & .476 & 276 & 0.0000 \\
\hline CWBO 9 & .375 & 276 & 0.0000 \\
\hline CWBO 10 & .552 & 276 & 0.0000 \\
\hline CWBO 11 & .486 & 276 & 0.0000 \\
\hline Mean of Counterproductive Work Behaviors (Ind.; CWBI) Items & .612 & 281 & 0.0000 \\
\hline CWBI 1 & .657 & 276 & 0.0000 \\
\hline CWBI 2 & .541 & 276 & 0.0000 \\
\hline CWBI 3 & .397 & 276 & 0.0000 \\
\hline CWBI 4 & .562 & 276 & 0.0000 \\
\hline CWBI 5 & .504 & 276 & 0.0000 \\
\hline CWBI 6 & .559 & 276 & 0.0000 \\
\hline CWBI 7 & .417 & 276 & 0.0000 \\
\hline
\end{tabular}

Note. $\mathrm{S}-\mathrm{W}=$ Shapiro-Wilk Statistic $(\mathrm{W}) ; \mathrm{Df}=$ Degrees of freedom; Sig. = p-value.; " p>.00042 (Bonferroni-corrected p-value) 
Table 9a. Direct and Indirect Effects of AS on Task Performance via PA (Hypothesis 1a).

\begin{tabular}{lcccc}
\hline \multicolumn{1}{c}{ Model } & $B$ & Boot SE & \multicolumn{2}{c}{ Boot 95\% CI } \\
& & & LLCI & ULCI \\
\hline Model A1 (DV=PA) & & & & \\
Direct effect of AS (Path $a$ ) & -.150 & .078 & -.314 & -.010 \\
Model A2 (DV= Task) & & & & \\
Direct effect of AS (Path $c$ ) & -.363 & .061 & -.489 & -.253 \\
Direct effect of PA (Path $b$ ) & .000 & .044 & -.083 & .089 \\
Model A3 (DV = Task) & .000 & .008 & -.014 & .018 \\
$\quad$ Indirect effect of AS via PA (Path $a b)$ & & &
\end{tabular}

Table 9b. Direct and Indirect Effects of AS on OCBO via PA (Hypothesis 1b).

\begin{tabular}{|c|c|c|c|c|}
\hline \multirow[t]{2}{*}{ Model } & \multirow[t]{2}{*}{$B$} & \multirow[t]{2}{*}{ Boot SE } & \multicolumn{2}{|c|}{ Boot $95 \%$ CI } \\
\hline & & & LLCI & ULCI \\
\hline Model B1 $(\mathrm{DV}=\mathrm{PA})$ & & & & \\
\hline Direct effect of AS (Path $a$ ) & -.150 & .078 & -.315 & -.013 \\
\hline Model B2 $(\mathrm{DV}=\mathrm{OCBO})$ & & & & \\
\hline Direct effect of AS (Path $c^{\prime}$ ) & -.128 & .063 & -.260 & -.009 \\
\hline Direct effect of PA (Path $b$ ) & .274 & .058 & .159 & .388 \\
\hline Model B3 $(\mathrm{DV}=\mathrm{OCBO})$ & & & & \\
\hline Indirect effect of AS via PA (Path $a b$ ) & -.041 & .022 & -.090 & -.004 \\
\hline
\end{tabular}

Table 9c. Direct and Indirect Effects of AS on OCBI via PA (Hypothesis 1c).

\begin{tabular}{lcccc}
\hline \multicolumn{1}{c}{ Model } & $B$ & Boot SE & \multicolumn{2}{c}{ Boot 95\% CI } \\
& & & LLCI & ULCI \\
\hline Model C1 (DV=PA) & & & & \\
Direct effect of AS (Path $a$ ) & -.150 & .079 & -.316 & -.009 \\
Model C2 (DV= OCBI) & & & & \\
Direct effect of AS (Path $c$ ') & -.072 & .058 & -.187 & .039 \\
Direct effect of PA (Path $b$ ) & .195 & .057 & .085 & .304 \\
Model C3 (DV = OCBI) & -.029 & .017 & -.068 & -.002 \\
$\quad$ Indirect effect of AS via PA (Path $a b)$ & & & \\
Note. $N=281 ; B=$ unstandardized coefficient; $S E=$ Standard error; CI= Confidence interval, AS = \\
Abusive supervision; PA = Positive affect (state). Boot= Bootstrap based on 5000 samples. Boot \\
95\% CIs constructed using percentile method.
\end{tabular}


Table 9d. Direct and Indirect Effects of AS on CWBO via PA (Hypothesis 1d).

\begin{tabular}{|c|c|c|c|c|}
\hline \multirow[t]{2}{*}{ Model } & \multirow[t]{2}{*}{$B$} & \multirow[t]{2}{*}{ Boot SE } & \multicolumn{2}{|c|}{ Boot 95\% CI } \\
\hline & & & LLCI & ULCI \\
\hline \multicolumn{5}{|l|}{ Model D1 (DV=PA) } \\
\hline Direct effect of AS (Path $a$ ) & -.150 & .077 & -.317 & -.009 \\
\hline \multicolumn{5}{|l|}{ Model D2 $(\mathrm{DV}=\mathrm{CWBO})$} \\
\hline Direct effect of AS (Path $c^{\prime}$ ) & .616 & .073 & .468 & .756 \\
\hline Direct effect of PA (Path $b$ ) & .149 & .049 & .052 & .246 \\
\hline \multicolumn{5}{|l|}{ Model D3 (DV = CWBO) } \\
\hline Indirect effect of AS via PA (Path $a b$ ) & -.022 & .014 & -.056 & -.001 \\
\hline
\end{tabular}

Table 9e. Direct and Indirect Effects of AS on CWBI via PA (Hypothesis 1e).

\begin{tabular}{ccccc}
\hline \multicolumn{1}{c}{ Model } & $B$ & Boot SE & \multicolumn{2}{c}{ Boot 95\% CI } \\
& & & & \\
& & & & \\
Model E1 (DV=PA) & -.150 & .080 & -.318 & -.006 \\
Direct effect of AS (Path $a)$ & & & & \\
Model E2 (DV= CWBI) & .595 & .077 & .440 & .742 \\
$\quad$ Direct effect of AS (Path $c^{\prime}$ ) & .157 & .043 & .069 & .237 \\
$\quad$ Direct effect of PA (Path $b$ ) & & & & \\
Model E3 (DV = CWBI) & -.024 & .015 & -.058 & -.001 \\
$\quad$ Indirect effect of AS via PA (Path $a b)$ &
\end{tabular}

Note. $N=281 ; B=$ unstandardized coefficient; $S E=$ Standard error; $\mathrm{CI}=$ Confidence interval, $\mathrm{AS}=$ Abusive supervision; PA $=$ Positive affect (state). Boot $=$ Bootstrap based on 5000 samples. Boot $95 \%$ CIs constructed using percentile method.

Table 10a. Direct and Indirect Effects of AS on Task Performance via NA (Hypothesis 2a).

\begin{tabular}{|c|c|c|c|c|}
\hline \multirow[t]{2}{*}{ Model } & \multirow[t]{2}{*}{$B$} & \multirow[t]{2}{*}{ Boot SE } & \multicolumn{2}{|c|}{ Boot 95\% CI } \\
\hline & & & LLCI & ULCI \\
\hline Model A1 (DV=NA) & & & & \\
\hline Direct effect of AS (Path $a$ ) & .608 & .075 & .462 & .755 \\
\hline Model A2 (DV= Task) & & & & \\
\hline Direct effect of AS (Path $c^{\prime}$ ) & -.379 & .067 & -.524 & -.259 \\
\hline Direct effect of NA (Path $b$ ) & .026 & .051 & -.072 & .128 \\
\hline Model A3 (DV = Task) & & & & \\
\hline Indirect effect of AS via NA (Path $a b$ ) & .016 & .032 & -.041 & .085 \\
\hline
\end{tabular}

Note. $N=281 ; B=$ unstandardized coefficient; $S E=$ Standard error; $\mathrm{CI}=$ Confidence interval, $\mathrm{AS}=$ Abusive supervision; NA = Negative affect (state). Boot= Bootstrap based on 5000 samples. Boot 95\% CIs constructed using percentile method. 
Table 10b. Direct and Indirect Effects of AS on OCBO via NA (Hypothesis 2b).

\begin{tabular}{|c|c|c|c|c|}
\hline \multirow[t]{2}{*}{ Model } & \multirow[t]{2}{*}{$B$} & \multirow[t]{2}{*}{ Boot SE } & \multicolumn{2}{|c|}{ Boot 95\% CI } \\
\hline & & & LLCI & ULCI \\
\hline \multicolumn{5}{|l|}{ Model B1 (DV=NA) } \\
\hline Direct effect of AS (Path $a$ ) & .608 & .074 & .457 & .748 \\
\hline \multicolumn{5}{|l|}{ Model B2 $(\mathrm{DV}=\mathrm{OCBO})$} \\
\hline Direct effect of AS (Path $c^{\prime}$ ) & -.199 & .071 & -.348 & -.070 \\
\hline Direct effect of NA (Path $b$ ) & .048 & .067 & -.083 & .179 \\
\hline \multicolumn{5}{|l|}{ Model B3 $(\mathrm{DV}=\mathrm{OCBO})$} \\
\hline Indirect effect of AS via NA (Path $a b$ ) & .029 & .042 & -.049 & .116 \\
\hline
\end{tabular}

Table 10c. Direct and Indirect Effects of AS on OCBI via NA (Hypothesis 2c).

\begin{tabular}{lcccc}
\hline Model & $B$ & Boot SE & \multicolumn{2}{c}{ Boot 95\% CI } \\
& & & LLCI & ULCI \\
\hline Model C1 (DV=NA) & & & & \\
Direct effect of AS (Path $a$ ) & .608 & .076 & .457 & .749 \\
Model C2 (DV= OCBI) & & & & \\
Direct effect of AS (Path $c^{\prime}$ ) & -.138 & .065 & -.265 & -.012 \\
Direct effect of NA (Path $b$ ) & .060 & .068 & -.072 & .190 \\
Model C3 (DV = OCBI) & .037 & .043 & -.045 & .124 \\
$\quad$ Indirect effect of AS via NA (Path $a b)$ & & & \\
Note. $N=281 ; B=$ unstandardized coefficient; $S E=$ Standard error; CI= Confidence interval, AS = \\
Abusive supervision; NA = Negative affect (state). Boot= Bootstrap based on 5000 samples. Boot \\
95\% CIs constructed using percentile method.
\end{tabular}

Table 10d. Direct and Indirect Effects of AS on CWBO via NA (Hypothesis 2d).

\begin{tabular}{|c|c|c|c|c|}
\hline \multirow[t]{2}{*}{ Model } & \multirow[t]{2}{*}{$B$} & \multirow[t]{2}{*}{ Boot SE } & \multicolumn{2}{|c|}{ Boot $95 \%$ CI } \\
\hline & & & LLCI & ULCI \\
\hline Model D1 (DV=NA) & & & & \\
\hline Direct effect of AS (Path $a$ ) & .608 & .076 & .458 & .749 \\
\hline Model D2 (DV $=\mathrm{CWBO})$ & & & & \\
\hline Direct effect of AS (Path $c^{\prime}$ ) & .561 & .090 & .380 & .735 \\
\hline Direct effect of NA (Path $b$ ) & .054 & .064 & -.065 & .182 \\
\hline Model D3 (DV = CWBO) & & & & \\
\hline Indirect effect of AS via NA (Path $a b$ ) & .033 & .038 & -.042 & .106 \\
\hline
\end{tabular}


Table 10e. Direct and Indirect Effects of AS on CWBI via NA (Hypothesis 2e).

\begin{tabular}{|c|c|c|c|c|}
\hline \multirow[t]{2}{*}{ Model } & \multirow[t]{2}{*}{$B$} & \multirow[t]{2}{*}{ Boot SE } & \multicolumn{2}{|c|}{ Boot 95\% CI } \\
\hline & & & LLCI & ULCI \\
\hline \multicolumn{5}{|l|}{ Model E1 (DV=NA) } \\
\hline Direct effect of AS (Path $a)$ & .608 & .075 & .458 & .751 \\
\hline \multicolumn{5}{|l|}{ Model E2 (DV= CWBI) } \\
\hline Direct effect of AS (Path $c^{\prime}$ ) & .536 & .085 & .371 & .704 \\
\hline Direct effect of NA (Path $b$ ) & .059 & .056 & -.051 & .166 \\
\hline \multicolumn{5}{|l|}{ Model E3 (DV = CWBI) } \\
\hline Indirect effect of AS via NA (Path $a b$ ) & .036 & .033 & -.034 & .097 \\
\hline
\end{tabular}

Table 11a. Direct and Indirect Effects of AS on Task Performance via LMX (Hypothesis 3a).

\begin{tabular}{|c|c|c|c|c|}
\hline \multirow[t]{2}{*}{ Model } & \multirow[t]{2}{*}{$B$} & \multirow[t]{2}{*}{ Boot SE } & \multicolumn{2}{|c|}{ Boot $95 \%$ CI } \\
\hline & & & LLCI & ULCI \\
\hline Model A1 (DV= LMX) & & & & \\
\hline Direct effect of AS (Path $a$ ) & -.538 & .073 & -.690 & -.406 \\
\hline Model A2 (DV= Task) & & & & \\
\hline Direct effect of AS (Path $c^{\prime}$ ) & -.341 & .065 & -.482 & -.221 \\
\hline Direct effect of LMX (Path $b$ ) & .041 & .048 & -.051 & .138 \\
\hline Model A3 (DV = Task $)$ & & & & \\
\hline Indirect effect of AS via LMX (Path $a b$ ) & -.022 & .026 & -.074 & .029 \\
\hline
\end{tabular}

Note. $N=281 ; B=$ unstandardized coefficient; $S E=$ Standard error; $\mathrm{CI}=$ Confidence interval, AS

$=$ Abusive supervision; LMX $=$ Leader-Member Exchange. Boot $=$ Bootstrap based on 5000 samples. Boot $95 \%$ CIs constructed using percentile method.

Table 11b. Direct and Indirect Effects of AS on OCBO via LMX (Hypothesis 3b).

\begin{tabular}{|c|c|c|c|c|}
\hline \multirow[t]{2}{*}{ Model } & \multirow[t]{2}{*}{$B$} & \multirow[t]{2}{*}{ Boot SE } & \multicolumn{2}{|c|}{ Boot $95 \%$ CI } \\
\hline & & & LLCI & ULCI \\
\hline Model B1 (DV= LMX) & & & & \\
\hline Direct effect of AS (Path $a$ ) & -.538 & .070 & -.686 & -.411 \\
\hline Model B2 (DV= OCBO) & & & & \\
\hline Direct effect of AS (Path $c^{\prime}$ ) & -.005 & .070 & -.152 & .121 \\
\hline Direct effect of LMX (Path $b$ ) & .305 & .065 & .172 & .426 \\
\hline Model B3 $(\mathrm{DV}=\mathrm{OCBO})$ & & & & \\
\hline Indirect effect of AS via LMX (Path $a b$ ) & -.164 & .041 & -.252 & -.089 \\
\hline
\end{tabular}

Note. $N=281 ; B=$ unstandardized coefficient; $S E=$ Standard error; $\mathrm{CI}=$ Confidence interval, $\mathrm{AS}=$ Abusive supervision; LMX $=$ Leader-Member Exchange. Boot $=$ Bootstrap based on 5000 samples. Boot $95 \%$ CIs constructed using percentile method. 
Table 11c. Direct and Indirect Effects of AS on OCBI via LMX (Hypothesis 3c).

\begin{tabular}{|c|c|c|c|c|}
\hline \multirow[t]{2}{*}{ Model } & \multirow[t]{2}{*}{$B$} & \multirow[t]{2}{*}{ Boot SE } & \multicolumn{2}{|c|}{ Boot 95\% CI } \\
\hline & & & LLCI & ULCI \\
\hline \multicolumn{5}{|l|}{ Model C1 (DV= LMX) } \\
\hline Direct effect of AS (Path $a$ ) & -.538 & .072 & -.696 & -.412 \\
\hline \multicolumn{5}{|l|}{ Model C2 (DV= OCBI) } \\
\hline Direct effect of AS (Path $c^{\prime}$ ) & .035 & .062 & -.089 & .157 \\
\hline Direct effect of LMX (Path $b$ ) & .254 & .066 & .122 & .384 \\
\hline \multicolumn{5}{|l|}{ Model C3 $(\mathrm{DV}=\mathrm{OCBI})$} \\
\hline Indirect effect of AS via LMX (Path $a b$ ) & -.137 & .041 & -.224 & -.065 \\
\hline
\end{tabular}

Table 11d. Direct and Indirect Effects of AS on CWBO via LMX (Hypothesis 3d).

\begin{tabular}{|c|c|c|c|c|}
\hline \multirow[t]{2}{*}{ Model } & \multirow[t]{2}{*}{$B$} & \multirow[t]{2}{*}{ Boot SE } & \multicolumn{2}{|c|}{ Boot 95\% CI } \\
\hline & & & LLCI & ULCI \\
\hline Model D1 (DV= LMX) & & & & \\
\hline Direct effect of AS (Path $a$ ) & -.538 & .070 & -.688 & -.409 \\
\hline Model D2 (DV=CWBO) & & & & \\
\hline Direct effect of AS (Path $c^{\prime}$ ) & .701 & .077 & .538 & .837 \\
\hline Direct effect of LMX (Path $b$ ) & .199 & .052 & .096 & .301 \\
\hline Model D3 (DV = CWBO) & & & & \\
\hline Indirect effect of AS via LMX (Path $a b$ ) & -.107 & .030 & -.170 & -.050 \\
\hline
\end{tabular}

Table 11e. Direct and Indirect Effects of AS on CWBI via LMX (Hypothesis 3e).

\begin{tabular}{|c|c|c|c|c|}
\hline \multirow{2}{*}{ Model } & \multirow[t]{2}{*}{$B$} & \multirow[t]{2}{*}{ Boot SE } & \multicolumn{2}{|c|}{ Boot 95\% CI } \\
\hline & & & LLCI & ULCI \\
\hline Model E1 (DV= LMX) & & & & \\
\hline Direct effect of AS (Path $a$ ) & -.538 & .072 & -.687 & -.406 \\
\hline Model E2 $(\mathrm{DV}=\mathrm{CWBI})$ & & & & \\
\hline Direct effect of AS (Path $c^{\prime}$ ) & .671 & .081 & .502 & .820 \\
\hline Direct effect of LMX (Path $b$ ) & .185 & .056 & .073 & .291 \\
\hline Model E3 $(\mathrm{DV}=\mathrm{CWBI})$ & & & & \\
\hline Indirect effect of AS via LMX (Path $a b$ ) & -.100 & .032 & -.166 & -.039 \\
\hline
\end{tabular}


Table 12a. Direct and Indirect Effects of AS on Task Performance via IJ (Hypothesis 4a).

\begin{tabular}{ccccc}
\hline \multicolumn{1}{c}{ Model } & $B$ & Boot SE & \multicolumn{2}{c}{ Boot 95\% CI } \\
& & & & \\
LLCI & ULCI \\
\hline Model A1 (DV= IJ) & & & & \\
Direct effect of AS (Path $a)$ & -.582 & .083 & -.750 & -.430 \\
Model A2 (DV= Task) & & & & \\
Direct effect of AS (Path $c^{\prime}$ ) & -.341 & .066 & -.482 & -.222 \\
$\quad$ Direct effect of IJ (Path $b$ ) & .037 & .059 & -.069 & .163 \\
Model A3 (DV = Task) & & & & \\
Indirect effect of AS via IJ (Path $a b)$ & -.022 & .034 & -.090 & .045 \\
\hline
\end{tabular}

Note. $N=281 ; B=$ unstandardized coefficient; $S E=$ Standard error; $\mathrm{CI}=$ Confidence interval, $\mathrm{AS}=$ Abusive supervision; IJ = Interactional Justice. Boot= Bootstrap based on 5000 samples. Boot 95\% CIs constructed using percentile method.

Table 12b. Direct and Indirect Effects of AS on OCBO via IJ (Hypothesis 4b).

\begin{tabular}{lcccc}
\hline \multicolumn{1}{c}{ Model } & $B$ & Boot SE & \multicolumn{2}{c}{ Boot 95\% CI } \\
& & & & \\
& & & & \\
& & & & \\
Model B1 (DV= IJ) & -.582 & .083 & -.759 & -.432 \\
Direct effect of AS (Path $a)$ & & & & \\
Model B2 (DV= OCBO) & .014 & .068 & -.129 & .142 \\
Direct effect of AS (Path $c^{\prime}$ ) & .314 & .077 & .160 & .465 \\
$\quad$ Direct effect of IJ (Path $b$ ) & & & & \\
Model B3 (DV = OCBO) & -.183 & .050 & -.288 & -.092 \\
$\quad$ Indirect effect of AS via IJ (Path $a b)$ &
\end{tabular}

Note. $N=281 ; B=$ unstandardized coefficient; $S E=$ Standard error; $\mathrm{CI}=$ Confidence interval, $\mathrm{AS}=$ Abusive supervision; IJ = Interactional Justice. Boot= Bootstrap based on 5000 samples. Boot 95\% CIs constructed using percentile method.

Table 12c. Direct and Indirect Effects of AS on OCBI via IJ (Hypothesis 4c).

\begin{tabular}{|c|c|c|c|c|}
\hline \multirow[t]{2}{*}{ Model } & \multirow[t]{2}{*}{$B$} & \multirow[t]{2}{*}{ Boot SE } & \multicolumn{2}{|c|}{ Boot 95\% CI } \\
\hline & & & LLCI & ULCI \\
\hline Model C1 (DV= IJ) & & & & \\
\hline Direct effect of AS (Path $a$ ) & -.582 & .084 & -.758 & -.427 \\
\hline Model C2 (DV= OCBI) & & & & \\
\hline Direct effect of AS (Path $c^{\prime}$ ) & .012 & .063 & -.117 & .131 \\
\hline Direct effect of IJ (Path $b$ ) & .195 & .076 & .051 & .342 \\
\hline Model C3 (DV = OCBI) & & & & \\
\hline Indirect effect of AS via IJ (Path $a b$ ) & -.113 & .046 & -.206 & -.030 \\
\hline
\end{tabular}

Note. $N=281 ; B=$ unstandardized coefficient; $S E=$ Standard error; $\mathrm{CI}=$ Confidence interval, $\mathrm{AS}=$ Abusive supervision; IJ = Interactional Justice. Boot= Bootstrap based on 5000 samples. Boot 95\% CIs constructed using percentile method. 
Table 12d. Direct and Indirect Effects of AS on CWBO via IJ (Hypothesis 4d).

\begin{tabular}{|c|c|c|c|c|}
\hline \multirow[t]{2}{*}{ Model } & \multirow[t]{2}{*}{$B$} & \multirow[t]{2}{*}{ Boot SE } & \multicolumn{2}{|c|}{ Boot 95\% CI } \\
\hline & & & LLCI & ULCI \\
\hline \multicolumn{5}{|l|}{ Model D1 (DV= IJ) } \\
\hline Direct effect of AS (Path $a$ ) & -.582 & .081 & -.747 & -.425 \\
\hline \multicolumn{5}{|l|}{ Model D2 (DV= CWBO) } \\
\hline Direct effect of AS (Path $c^{\prime}$ ) & .735 & .073 & .581 & .873 \\
\hline Direct effect of IJ (Path $b$ ) & .242 & .046 & .146 & .327 \\
\hline \multicolumn{5}{|l|}{ Model D3 (DV = CWBO) } \\
\hline Indirect effect of AS via IJ (Path $a b$ ) & -.141 & .032 & -.205 & -.080 \\
\hline
\end{tabular}

Table 12e. Direct and Indirect Effects of AS on CWBI via IJ (Hypothesis 4e).

\begin{tabular}{|c|c|c|c|c|}
\hline \multirow[t]{2}{*}{ Model } & \multirow[t]{2}{*}{$B$} & \multirow[t]{2}{*}{ Boot SE } & \multicolumn{2}{|c|}{ Boot $95 \%$ CI } \\
\hline & & & LLCI & ULCI \\
\hline \multicolumn{5}{|l|}{ Model E1 $(\mathrm{DV}=\mathrm{IJ})$} \\
\hline Direct effect of AS (Path $a$ ) & -.582 & .083 & -.754 & -.433 \\
\hline \multicolumn{5}{|l|}{ Model E2 (DV= CWBI) } \\
\hline Direct effect of AS (Path $c^{\prime}$ ) & .689 & .081 & .516 & .832 \\
\hline Direct effect of IJ (Path $b$ ) & .201 & .051 & .098 & .301 \\
\hline \multicolumn{5}{|l|}{ Model E3 $(\mathrm{DV}=\mathrm{CWBI})$} \\
\hline Indirect effect of AS via IJ (Path $a b$ ) & -.117 & .034 & -.188 & -.054 \\
\hline
\end{tabular}

Table 13a. Direct and Indirect Effects of AS on Task Performance via POS (Hypothesis 5a).

\begin{tabular}{|c|c|c|c|c|}
\hline \multirow[t]{2}{*}{ Model } & \multirow[t]{2}{*}{$B$} & \multirow[t]{2}{*}{ Boot SE } & \multicolumn{2}{|c|}{ Boot $95 \% \mathrm{CI}$} \\
\hline & & & LLCI & ULCI \\
\hline \multicolumn{5}{|l|}{ Model A1 $(\mathrm{DV}=$ POS $)$} \\
\hline Direct effect of AS (Path $a$ ) & -.404 & .068 & -.554 & -.286 \\
\hline \multicolumn{5}{|l|}{ Model A2 (DV= Task) } \\
\hline Direct effect of AS (Path $c^{\prime}$ ) & -.371 & .064 & -.507 & -.260 \\
\hline Direct effect of POS (Path $b$ ) & -.020 & .043 & -.102 & .068 \\
\hline \multicolumn{5}{|l|}{ Model A3 $(\mathrm{DV}=$ Task $)$} \\
\hline Indirect effect of AS via POS (Path $a b$ ) & .008 & .018 & -.026 & .047 \\
\hline
\end{tabular}


Table 13b. Direct and Indirect Effects of AS on OCBO via POS (Hypothesis 5b).

\begin{tabular}{|c|c|c|c|c|}
\hline \multirow[t]{2}{*}{ Model } & \multirow[t]{2}{*}{$B$} & \multirow[t]{2}{*}{ Boot SE } & \multicolumn{2}{|c|}{ Boot $95 \% \mathrm{CI}$} \\
\hline & & & LLCI & ULCI \\
\hline \multicolumn{5}{|l|}{ Model B1 $(\mathrm{DV}=$ POS $)$} \\
\hline Direct effect of AS (Path $a$ ) & -.404 & .068 & -.551 & -.287 \\
\hline \multicolumn{5}{|l|}{ Model B2 (DV= OCBO) } \\
\hline Direct effect of AS (Path $c^{\prime}$ ) & -.092 & .068 & -.232 & .030 \\
\hline Direct effect of POS (Path $b$ ) & .191 & .070 & .057 & .326 \\
\hline \multicolumn{5}{|l|}{ Model B3 (DV = OCBO) } \\
\hline Indirect effect of AS via POS (Path $a b$ ) & -.077 & .030 & -.140 & -.023 \\
\hline
\end{tabular}

Table 13c. Direct and Indirect Effects of AS on OCBI via POS (Hypothesis 5c).

\begin{tabular}{|c|c|c|c|c|}
\hline \multirow[t]{2}{*}{ Model } & \multirow[t]{2}{*}{$B$} & \multirow[t]{2}{*}{ Boot SE } & \multicolumn{2}{|c|}{ Boot $95 \%$ CI } \\
\hline & & & LLCI & ULCI \\
\hline Model C1 $(\mathrm{DV}=$ POS $)$ & & & & \\
\hline Direct effect of AS (Path $a$ ) & -.404 & .069 & -.555 & -.285 \\
\hline Model C2 (DV= OCBI) & & & & \\
\hline Direct effect of AS (Path $c^{\prime}$ ) & -.030 & .061 & -.154 & .088 \\
\hline Direct effect of POS (Path $b$ ) & .176 & .070 & .041 & .316 \\
\hline Model C3 $(\mathrm{DV}=\mathrm{OCBI})$ & & & & \\
\hline Indirect effect of AS via POS (Path $a b$ ) & -.071 & .030 & -.135 & -.017 \\
\hline
\end{tabular}

Table 13d. Direct and Indirect Effects of AS on CWBO via POS (Hypothesis 5d).

\begin{tabular}{|c|c|c|c|c|}
\hline \multirow[t]{2}{*}{ Model } & \multirow[t]{2}{*}{$B$} & \multirow[t]{2}{*}{ Boot SE } & \multicolumn{2}{|c|}{ Boot $95 \%$ CI } \\
\hline & & & LLCI & ULCI \\
\hline \multicolumn{5}{|l|}{ Model D1 $(\mathrm{DV}=$ POS $)$} \\
\hline Direct effect of AS (Path $a$ ) & -.404 & .068 & -.552 & -.286 \\
\hline \multicolumn{5}{|l|}{ Model D2 (DV= CWBO) } \\
\hline Direct effect of AS (Path $c^{\prime}$ ) & .624 & .088 & .450 & .790 \\
\hline Direct effect of POS (Path $b$ ) & .076 & .069 & -.071 & .197 \\
\hline \multicolumn{5}{|l|}{ Model D3 $(\mathrm{DV}=\mathrm{CWBO})$} \\
\hline Indirect effect of AS via POS (Path $a b$ ) & -.031 & .030 & -.090 & .028 \\
\hline
\end{tabular}


Table 13e. Direct and Indirect Effects of AS on CWBI via POS (Hypothesis 5e).

\begin{tabular}{|c|c|c|c|c|}
\hline \multirow[t]{2}{*}{ Model } & \multirow[t]{2}{*}{$B$} & \multirow[t]{2}{*}{ Boot SE } & \multicolumn{2}{|c|}{ Boot $95 \% \mathrm{CI}$} \\
\hline & & & LLCI & ULCI \\
\hline \multicolumn{5}{|l|}{ Model E1 (DV= POS) } \\
\hline Direct effect of AS (Path $a$ ) & -.404 & .068 & -.556 & -.286 \\
\hline \multicolumn{5}{|l|}{ Model E2 (DV= CWBI) } \\
\hline Direct effect of AS (Path $c^{\prime}$ ) & .591 & .091 & .407 & .764 \\
\hline Direct effect of POS (Path $b$ ) & .048 & .069 & -.097 & .170 \\
\hline \multicolumn{5}{|l|}{ Model E3 (DV = CWBI) } \\
\hline Indirect effect of AS via POS (Path $a b$ ) & -.019 & .029 & -.074 & .038 \\
\hline
\end{tabular}

Table 14a. Direct and Indirect Effects of AS on Task Performance via AOC (Hypothesis 6a).

\begin{tabular}{|c|c|c|c|c|}
\hline \multirow[t]{2}{*}{ Model } & \multirow[t]{2}{*}{$B$} & \multirow[t]{2}{*}{ Boot SE } & \multicolumn{2}{|c|}{ Boot 95\% CI } \\
\hline & & & LLCI & ULCI \\
\hline Model A1 (DV= AOC) & & & & \\
\hline Direct effect of AS (Path $a$ ) & -.274 & .059 & -.398 & -.168 \\
\hline Model A2 (DV= Task) & & & & \\
\hline Direct effect of AS (Path $c^{\prime}$ ) & -.382 & .062 & -.512 & -.269 \\
\hline Direct effect of AOC (Path $b$ ) & -.069 & .037 & -.140 & .006 \\
\hline Model A3 (DV = Task) & & & & \\
\hline Indirect effect of AS via AOC (Path $a b)$ & .019 & .012 & -.002 & .045 \\
\hline
\end{tabular}

Note. $N=281 ; B=$ unstandardized coefficient; $S E=$ Standard error; $\mathrm{CI}=$ Confidence interval, $\mathrm{AS}=$ Abusive supervision; AOC $=$ Affective organizational commitment. Boot $=$ Bootstrap based on 5000 samples. Boot $95 \%$ CIs constructed using percentile method.

Table 14b. Direct and Indirect Effects of AS on OCBO via AOC (Hypothesis 6b).

\begin{tabular}{|c|c|c|c|c|}
\hline \multirow{2}{*}{ Model } & \multirow[t]{2}{*}{$B$} & \multirow{2}{*}{ Boot SE } & \multicolumn{2}{|c|}{ Boot $95 \%$ CI } \\
\hline & & & LLCI & ULCI \\
\hline Model B1 (DV= AOC) & & & & \\
\hline Direct effect of AS (Path $a$ ) & -.274 & .058 & -.399 & -.170 \\
\hline Model B2 $(\mathrm{DV}=\mathrm{OCBO})$ & & & & \\
\hline Direct effect of AS (Path $c^{\prime}$ ) & -.118 & .066 & -.253 & .004 \\
\hline Direct effect of AOC (Path $b$ ) & .186 & .061 & .068 & .306 \\
\hline Model B3 $(\mathrm{DV}=\mathrm{OCBO})$ & & & & \\
\hline Indirect effect of AS via AOC (Path $a b$ ) & -.051 & .021 & -.099 & -.016 \\
\hline
\end{tabular}


Table 14c. Direct and Indirect Effects of AS on OCBI via AOC (Hypothesis 6c).

\begin{tabular}{|c|c|c|c|c|}
\hline \multirow[t]{2}{*}{ Model } & \multirow[t]{2}{*}{$B$} & \multirow[t]{2}{*}{ Boot SE } & \multicolumn{2}{|c|}{ Boot $95 \% \mathrm{CI}$} \\
\hline & & & LLCI & ULCI \\
\hline Model C1 (DV= AOC) & & & & \\
\hline Direct effect of AS (Path $a$ ) & -.274 & .059 & -.401 & -.168 \\
\hline Model C2 $(\mathrm{DV}=\mathrm{OCBI})$ & & & & \\
\hline Direct effect of AS (Path $c^{\prime}$ ) & -.061 & .060 & -.179 & .054 \\
\hline Direct effect of AOC (Path $b$ ) & .148 & .061 & .031 & .270 \\
\hline Model C3 $(\mathrm{DV}=\mathrm{OCBI})$ & & & & \\
\hline Indirect effect of AS via AOC (Path $a b$ ) & -.040 & .020 & -.085 & -.008 \\
\hline
\end{tabular}

Note. $N=281 ; B=$ unstandardized coefficient; $S E=$ Standard error; $\mathrm{CI}=$ Confidence interval, $\mathrm{AS}=$ Abusive supervision; AOC $=$ Affective organizational commitment. Boot $=$ Bootstrap based on 5000 samples. Boot $95 \%$ CIs constructed using percentile method.

Table 14d. Direct and Indirect Effects of AS on CWBO via AOC (Hypothesis 6d).

\begin{tabular}{lcccr}
\hline \multicolumn{1}{c}{ Model } & $B$ & Boot SE & \multicolumn{2}{c}{ Boot 95\% CI } \\
& & & & \\
& LLCI & ULCI \\
\hline Model D1 (DV= AOC) & -.274 & .058 & -.398 & -.172 \\
Direct effect of AS (Path $a)$ & & & & \\
Model D2 (DV= CWBO) & .613 & .084 & .441 & .773 \\
Direct effect of AS (Path $c^{\prime}$ ) & .072 & .049 & -.035 & .157 \\
Direct effect of AOC (Path $b$ ) & & & & \\
Model D3 (DV = CWBO) & -.020 & .015 & -.052 & .009 \\
$\quad$ Indirect effect of AS via AOC (Path $a b)$ &
\end{tabular}
Note. $N=281 ; B=$ unstandardized coefficient; $S E=$ Standard error; $\mathrm{CI}=$ Confidence interval, $\mathrm{AS}=$ Abusive supervision; AOC $=$ Affective organizational commitment. Boot $=$ Bootstrap based on 5000 samples. Boot $95 \%$ CIs constructed using percentile method.

Table 14e. Direct and Indirect Effects of AS on CWBI via AOC (Hypothesis 6e).

\begin{tabular}{|c|c|c|c|c|}
\hline \multirow{2}{*}{ Model } & \multirow[t]{2}{*}{$B$} & \multirow[t]{2}{*}{ Boot SE } & \multicolumn{2}{|c|}{ Boot 95\% CI } \\
\hline & & & LLCI & ULCI \\
\hline Model E1 (DV=AOC) & & & & \\
\hline Direct effect of AS (Path $a$ ) & -.274 & .059 & -.403 & -.168 \\
\hline Model E2 $(\mathrm{DV}=\mathrm{CWBI})$ & & & & \\
\hline Direct effect of AS (Path $c^{\prime}$ ) & .592 & .085 & .422 & .752 \\
\hline Direct effect of AOC (Path $b$ ) & .075 & .048 & -.027 & .159 \\
\hline Model E3 $(\mathrm{DV}=\mathrm{CWBI})$ & & & & \\
\hline Indirect effect of AS via AOC (Path $a b$ ) & -.021 & .015 & -.052 & .007 \\
\hline
\end{tabular}


Table 15a. Indirect effects of abusive supervision on task performance.

\begin{tabular}{lccc}
\hline \multicolumn{1}{c}{ Mediator } & $\begin{array}{c}\text { Effect } \\
(a b)\end{array}$ & $\begin{array}{c}\text { Boot } \\
\text { SE }(a b)\end{array}$ & $\begin{array}{c}\text { Boot } \\
95 \% \text { CI }\end{array}$ \\
\hline Positive Affect & -.00 & .01 & $-.03, .02$ \\
Negative Affect & .01 & .04 & $-.07, .09$ \\
Affective Organizational Commitment & .04 & .02 & $.01, .08^{*}$ \\
Perceived Organizational Support & .00 & .03 & $-.05, .05$ \\
Leader-Member Exchange & -.05 & .05 & $-.15, .04$ \\
Interactional Justice & -.03 & .05 & $-.14, .08$ \\
\hline
\end{tabular}

Note. $N=281 ; \mathrm{CI}=$ confidence interval; ${ }^{*} \mathrm{CI}$ excludes zero. Boot $=$ based on 5000 samples. Boot CIs is based on bootstrap percentile method.

Table 15b. Indirect effects of abusive supervision on OCBO.

\begin{tabular}{lccc}
\hline \multicolumn{1}{c}{ Mediator } & $\begin{array}{c}\text { Effect } \\
(a b)\end{array}$ & $\begin{array}{c}\text { Boot } \\
\text { SE }(a b)\end{array}$ & $\begin{array}{c}\text { Boot } \\
95 \% \mathrm{CI}\end{array}$ \\
\hline Positive Affect & -.04 & .03 & $-.11,-.00^{*}$ \\
Negative Affect & .13 & .05 & $.04, .24^{*}$ \\
Affective Organizational Commitment & -.00 & .02 & $-.05, .04$ \\
Perceived Organizational Support & .04 & .04 & $-.03, .12$ \\
Leader-Member Exchange & -.05 & .05 & $-.16, .05$ \\
Interactional Justice & -.08 & .06 & $-.21, .05$ \\
\hline
\end{tabular}

Note. $N=281 ; \mathrm{CI}=$ confidence interval; ${ }^{*} \mathrm{CI}$ excludes zero. $\mathrm{Boot}=$ based on 5000 samples. Boot CIs is based on bootstrap percentile method.

Table 15c. Indirect effects of abusive supervision on OCBI.

\begin{tabular}{lccc}
\hline \multicolumn{1}{c}{ Mediator } & $\begin{array}{c}\text { Effect } \\
(a b)\end{array}$ & $\begin{array}{c}\text { Boot } \\
\text { SE }(a b)\end{array}$ & $\begin{array}{c}\text { Boot } \\
95 \% \mathrm{CI}\end{array}$ \\
\hline Positive Affect & -.03 & .02 & $-.08, .00$ \\
Negative Affect & .12 & .05 & $.02, .22^{*}$ \\
Affective Organizational Commitment & .00 & .02 & $-.04, .05$ \\
Perceived Organizational Support & -.02 & .04 & $-.10, .05$ \\
Leader-Member Exchange & -.10 & .06 & $-.22, .01$ \\
Interactional Justice & .04 & .06 & $-.08, .17$ \\
\hline
\end{tabular}

Note. $N=281 ; \mathrm{CI}=$ confidence interval; ${ }^{*} \mathrm{CI}$ excludes zero. $\mathrm{Boot}=$ based on 5000 samples. Boot CIs is based on bootstrap percentile method. 
Table 15d. Indirect effects of abusive supervision on CWBO.

\begin{tabular}{lccc}
\hline \multicolumn{1}{c}{ Mediator } & $\begin{array}{c}\text { Effect } \\
(a b)\end{array}$ & $\begin{array}{c}\text { Boot } \\
\text { SE }(a b)\end{array}$ & $\begin{array}{c}\text { Boot } \\
95 \% \mathrm{CI}\end{array}$ \\
\hline Positive Affect & -.02 & .02 & $-.06, .00$ \\
Negative Affect & .07 & .04 & $-.00, .15$ \\
Affective Organizational Commitment & .01 & .02 & $-.03, .05$ \\
Perceived Organizational Support & .06 & .05 & $-.02, .16$ \\
Leader-Member Exchange & -.03 & .05 & $-.13, .08$ \\
Interactional Justice & -.14 & .05 & $-.25,-.04^{*}$ \\
\hline
\end{tabular}

Note. $N=281$; $\mathrm{CI}=$ confidence interval; ${ }^{*} \mathrm{CI}$ excludes zero. $\mathrm{Boot}=$ based on 5000 samples. Boot CIs is based on bootstrap percentile method.

Table 15e. Indirect effects of abusive supervision on CWBI.

\begin{tabular}{lccc}
\hline \multicolumn{1}{c}{ Mediator } & $\begin{array}{c}\text { Effect } \\
(a b)\end{array}$ & $\begin{array}{c}\text { Boot } \\
\text { SE }(a b)\end{array}$ & $\begin{array}{c}\text { Boot } \\
95 \% \mathrm{CI}\end{array}$ \\
\hline Positive Affect & -.03 & .02 & $-.08,-.00^{*}$ \\
Negative Affect & .08 & .04 & $.01, .15^{*}$ \\
Affective Organizational Commitment & .00 & .02 & $-.04, .04$ \\
Perceived Organizational Support & .08 & .05 & $-.01, .18$ \\
Leader-Member Exchange & -.04 & .06 & $-.15, .07$ \\
Interactional Justice & -.09 & .05 & $-.19,-.00^{*}$ \\
\hline
\end{tabular}

Note. $N=281 ; \mathrm{CI}=$ confidence interval; ${ }^{*} \mathrm{CI}$ excludes zero. $\mathrm{Boot}=$ based on 5000 samples. Boot CIs is based on bootstrap percentile method.

Table 16. Contrasts between specific indirect effects associated with PA and AOC (Hypotheses 7a-7e).

\begin{tabular}{lcccc}
\hline \multicolumn{1}{c}{ Outcome } & Absolute Difference & Boot SE & \multicolumn{2}{c}{ Boot 95\% CI } \\
& $\left(\left|a_{P A} b_{P A}\right|-\left|a_{A O C} b_{A O C}\right|\right)$ & & LLCI & ULCI \\
\hline Task performance & -0.04 & 0.02 & -0.07 & 0.01 \\
OCBO & 0.04 & 0.03 & -0.02 & 0.08 \\
OCBI & 0.02 & 0.02 & -0.03 & 0.06 \\
CWBO & 0.01 & 0.02 & -0.02 & 0.04 \\
CWBI & 0.03 & 0.02 & -0.02 & 0.07 \\
\hline
\end{tabular}

Note. $N=281 ; \mathrm{CI}=$ confidence interval; Boot $=5000$ samples. Boot CIs based on percentile method. 
Table 17. Contrasts between specific indirect effects associated with PA and POS (Hypotheses 8a-8e).

\begin{tabular}{lcccc}
\hline \multicolumn{1}{c}{ Outcome } & Difference & Boot SE & \multicolumn{2}{c}{ Boot 95\% CI } \\
& $\left(\left|a_{P A} b_{P A}\right|-\left|a_{A P O S} b_{P O S}\right|\right)$ & & LLCI & ULCI \\
\hline Task performance & 0.00 & 0.02 & -0.05 & 0.02 \\
OCBO & 0.01 & 0.03 & -0.07 & 0.07 \\
OCBI & 0.01 & 0.03 & -0.08 & 0.06 \\
CWBO & -0.04 & 0.04 & -0.12 & 0.03 \\
CWBI & -0.05 & 0.04 & -0.14 & 0.03 \\
\hline
\end{tabular}

Note. $N=281 ; \mathrm{CI}=$ confidence interval; Boot $=5000$ samples. Boot CIs based on percentile method.

Table 18. Contrasts between specific indirect effects associated with PA and LMX (Hypotheses 9a-9e).

\begin{tabular}{lcccc}
\hline \multicolumn{1}{c}{ Outcome } & Difference & Boot SE & \multicolumn{2}{c}{ Boot 95\% CI } \\
& $\left(\left|a_{P A} b_{P A}\right|-\left|a_{L M X} b_{L M X}\right|\right)$ & & LLCI & ULCI \\
\hline Task performance & -0.04 & 0.04 & -0.14 & 0.01 \\
OCBO & 0.00 & 0.05 & -0.11 & 0.07 \\
OCBI & -0.07 & 0.06 & -0.19 & 0.03 \\
CWBO & -0.01 & 0.04 & -0.11 & 0.04 \\
CWBI & -0.01 & 0.04 & -0.12 & 0.05 \\
\hline
\end{tabular}

Note. $N=281 ; \mathrm{CI}=$ confidence interval; Boot $=5000$ samples. Boot $C$ Is based on percentile method.

Table 19. Contrasts between specific indirect effects associated with PA and IJ (Hypotheses 10a-10e).

\begin{tabular}{lcrrr}
\hline \multicolumn{1}{c}{ Outcome } & \multicolumn{2}{c}{ Difference } & Boot SE & \multicolumn{2}{c}{ Boot 95\% CI } \\
& $\left(\left|a_{P A} b_{P A}\right|-\left|a_{I J} b_{I J}\right|\right)$ & & LLCI & ULCI \\
\hline Task performance & -0.02 & 0.04 & -0.13 & 0.01 \\
OCBO & -0.04 & 0.06 & -0.18 & 0.06 \\
OCBI & -0.01 & 0.05 & -0.14 & 0.04 \\
CWBO & -0.11 & 0.05 & -0.22 & -0.01 \\
CWBI & -0.06 & 0.05 & -0.16 & 0.03 \\
\hline
\end{tabular}

Note. $N=281 ; \mathrm{CI}=$ confidence interval; Boot $=5000$ samples. Boot CIs based on percentile method. 
Table 20. Contrasts between specific indirect effects associated with NA and AOC (Hypotheses 11a-11e).

\begin{tabular}{lcccc}
\hline \multicolumn{1}{c}{ Outcome } & Difference & Boot SE & \multicolumn{2}{c}{ Boot 95\% CI } \\
& $\left(\left|a_{N A} b_{N A}\right|-\left|a_{A O C} b_{A O C}\right|\right)$ & & LLCI & ULCI \\
\hline Task performance & -0.03 & 0.03 & -0.06 & 0.06 \\
OCBO & 0.13 & 0.05 & 0.01 & 0.22 \\
OCBI & 0.11 & 0.05 & 0.00 & 0.21 \\
CWBO & 0.06 & 0.04 & -0.02 & 0.13 \\
CWBI & 0.08 & 0.04 & -0.01 & 0.14 \\
\hline
\end{tabular}

Note. $N=281 ; \mathrm{CI}=$ confidence interval; Boot $=5000$ samples. Boot CIs based on percentile method.

Table 21. Contrasts between specific indirect effects associated with NA and POS (Hypotheses 12a-12e).

\begin{tabular}{lcccc}
\hline \multicolumn{1}{c}{ Outcome } & Difference & Boot SE & \multicolumn{2}{c}{ Boot 95\% CI } \\
& $\left(\left|a_{N A} b_{N A}\right|-\left|a_{P O S} b_{P O S}\right|\right)$ & & LLCI & ULCI \\
\hline Task performance & 0.00 & 0.03 & -0.04 & 0.08 \\
OCBO & 0.09 & 0.06 & -0.02 & 0.21 \\
OCBI & 0.10 & 0.06 & -0.02 & 0.20 \\
CWBO & 0.01 & 0.06 & -0.12 & 0.12 \\
CWBI & 0.00 & 0.07 & -0.14 & 0.12 \\
\hline
\end{tabular}

Note. $N=281 ; \mathrm{CI}=$ confidence interval; Boot $=5000$ samples. Boot $C$ Is based on percentile method.

Table 22. Contrasts between specific indirect effects associated with NA and LMX (Hypotheses 13a-13e).

\begin{tabular}{lcccc}
\hline \multicolumn{1}{c}{ Outcome } & Difference & Boot SE & \multicolumn{2}{c}{ Boot 95\% CI } \\
& $\left(\left|a_{N A} b_{N A}\right|-\left|a_{L M X} b_{L M X}\right|\right)$ & & LLCI & ULCI \\
\hline Task performance & -0.04 & 0.05 & -0.12 & 0.06 \\
OCBO & 0.08 & 0.07 & -0.07 & 0.21 \\
OCBI & 0.02 & 0.08 & -0.14 & 0.17 \\
CWBO & 0.04 & 0.05 & -0.09 & 0.12 \\
CWBI & 0.04 & 0.06 & -0.10 & 0.13 \\
\hline
\end{tabular}

Note. $N=281 ; \mathrm{CI}=$ confidence interval; Boot $=5000$ samples. Boot CIs based on percentile method. 
Table 23. Contrasts between specific indirect effects associated with NA and IJ (Hypotheses 14a-14e).

\begin{tabular}{lcccc}
\hline \multicolumn{1}{c}{ Outcome } & \multicolumn{2}{c}{ Difference } & Boot SE & \multicolumn{2}{c}{ Boot 95\% CI } \\
& $\left(\left|a_{N A} b_{N A}\right|-\left|a_{I J} b_{I J}\right|\right)$ & & LLCI & ULCI \\
\hline Task performance & -0.02 & 0.04 & -0.11 & 0.06 \\
OCBO & 0.05 & 0.08 & -0.11 & 0.19 \\
OCBI & 0.08 & 0.07 & -0.09 & 0.19 \\
CWBO & -0.07 & 0.07 & -0.20 & 0.07 \\
CWBI & -0.01 & 0.06 & -0.14 & 0.11 \\
\hline
\end{tabular}

Note. $N=281 ; \mathrm{CI}=$ confidence interval; Boot $=5000$ samples. Boot CIs based on percentile method.

Table 24. Contrasts between specific indirect effects associated with AOC and LMX (Hypotheses 15a-15e).

\begin{tabular}{lcccc}
\hline \multicolumn{1}{c}{ Outcome } & \multicolumn{2}{c}{ Difference } & Boot SE & \multicolumn{2}{c}{ Boot 95\% CI } \\
& $\left(\left|a_{A O C} b_{A O C}\right|-\left|a_{L M X} b_{L M X}\right|\right)$ & & LLCI & ULCI \\
\hline Task performance & -0.01 & 0.04 & -0.11 & 0.05 \\
OCBO & 0.05 & 0.08 & -0.11 & 0.19 \\
OCBI & -0.09 & 0.06 & -0.20 & 0.01 \\
CWBO & -0.02 & 0.04 & -0.11 & 0.03 \\
CWBI & -0.04 & 0.04 & -0.14 & 0.02 \\
\hline
\end{tabular}

Note. $N=281 ; \mathrm{CI}=$ confidence interval; Boot $=5000$ samples. Boot $\mathrm{CIs}$ based on percentile method.

Table 25. Contrasts between specific indirect effects associated with AOC and IJ (Hypotheses 16a-16e).

\begin{tabular}{lcccc}
\hline \multicolumn{1}{c}{ Outcome } & \multicolumn{2}{c}{ Difference } & Boot SE & \multicolumn{2}{c}{ Boot 95\% CI } \\
& $\left(\left|a_{A O C} b_{I J}\right|-\left|a_{L M X} b_{I J}\right|\right)$ & & LLCI & ULCI \\
\hline Task performance & 0.01 & 0.04 & -0.10 & 0.05 \\
OCBO & -0.08 & 0.06 & -0.20 & 0.02 \\
OCBI & -0.04 & 0.05 & -0.16 & 0.03 \\
CWBO & -0.13 & 0.05 & -0.23 & -0.02 \\
CWBI & -0.09 & 0.05 & -0.18 & 0.00 \\
\hline
\end{tabular}

Note. $N=281 ; \mathrm{CI}=$ confidence interval; $\mathrm{Boot}=5000$ samples. Boot $\mathrm{CIs}$ based on percentile method. 
Table 26. Contrasts between specific indirect effects associated with POS and LMX (Hypotheses 17a-17e).

\begin{tabular}{lcccc}
\hline \multicolumn{1}{c}{ Outcome } & \multicolumn{2}{c}{ Difference } & Boot SE & \multicolumn{2}{c}{ Boot 95\% CI } \\
& $\left(\left|a_{P O S} b_{P O S}\right|-\left|a_{L M X} b_{L M X}\right|\right)$ & & LLCI & ULCI \\
\hline Task performance & -0.04 & 0.04 & -0.13 & 0.03 \\
OCBO & -0.01 & 0.05 & -0.12 & 0.08 \\
OCBI & -0.08 & 0.06 & -0.19 & 0.05 \\
CWBO & 0.03 & 0.04 & -0.07 & 0.10 \\
CWBI & 0.04 & 0.05 & -0.06 & 0.12 \\
\hline
\end{tabular}

Note. $N=281 ; \mathrm{CI}=$ confidence interval; Boot $=5000$ samples. Boot CIs based on percentile method.

Table 27. Contrasts between specific indirect effects associated with POS and IJ (Hypotheses 18a-18e).

\begin{tabular}{lcccc}
\hline \multicolumn{1}{c}{ Outcome } & \multicolumn{2}{c}{ Difference } & Boot SE & \multicolumn{2}{c}{ Boot 95\% CI } \\
& $\left(\left|a_{P O S} b_{P O S}\right|-\left|a_{I J} b_{I J}\right|\right)$ & & LLCI & ULCI \\
\hline Task performance & -0.02 & 0.04 & -0.12 & 0.04 \\
OCBO & -0.04 & 0.06 & -0.17 & 0.05 \\
OCBI & -0.02 & 0.05 & -0.14 & 0.06 \\
CWBO & -0.08 & 0.05 & -0.19 & 0.02 \\
CWBI & -0.01 & 0.05 & -0.12 & 0.09 \\
\hline
\end{tabular}

Note. $N=281 ; \mathrm{CI}=$ confidence interval; Boot $=5000$ samples. Boot CIs based on percentile method.

Table 28a. Moderating effects of PEC on relationship between abusive supervision and LMX (Hypothesis 20a).

\begin{tabular}{llcccccccr}
\hline Model & Variable & $\mathrm{B}$ & $\begin{array}{c}\text { Boot } \\
\text { SE(B) }\end{array}$ & Sig.(B) & $\begin{array}{r}95 \% \\
\text { LLCI }\end{array}$ & $\begin{array}{r}\text { Boot } \\
\text { ULCI }\end{array}$ & $\mathrm{R}^{2}$ & $\Delta \mathrm{R}^{2}$ & Sig. $\left(\Delta \mathrm{R}^{2}\right)$ \\
\hline 1 & AS & -.20 & .01 & .000 & -.223 & -.172 & & & \\
& PEC & .79 & .03 & .000 & .732 & .855 & & & \\
& & & & & & & .33 & .33 & .000 \\
2 & AS & -.21 & .01 & .000 & -.231 & -.181 & & & \\
& PEC & .78 & .03 & .000 & .723 & .837 & & & \\
& AS*PEC & .16 & .03 & .000 & .099 & .125 & & & \\
& & & & & & & .34 & .01 & .000 \\
\hline
\end{tabular}

Note. $\mathrm{DV}=$ Leader-member exchange $(\mathrm{LMX}) . \mathrm{AS}=$ Abusive supervision; $\mathrm{PEC}=$ Perceived ethical climate. $\mathrm{B}=$ unstandardized coefficient, $\mathrm{SE}=$ Standard Error. Boot $=$ Bootstrap estimate based on 5000 samples. Boot CIs constructed with percentile method. 
Table 28b. Moderating effects of PEC on relationship between abusive supervision and IJ (Hypothesis 20b).

\begin{tabular}{llrrrrrrrr}
\hline Model & Variable & $\mathrm{B}$ & $\begin{array}{c}\text { Boot } \\
\text { SE(B) }\end{array}$ & Sig.(B) & $\begin{array}{r}95 \% \\
\text { LLCI }\end{array}$ & $\begin{array}{r}\text { Boot } \\
\text { ULCI }\end{array}$ & $\mathrm{R}^{2}$ & $\Delta \mathrm{R}^{2}$ & Sig. $\left(\Delta \mathrm{R}^{2}\right)$ \\
\hline 1 & AS & -.11 & .01 & .000 & -.136 & -.092 & & & \\
& PEC & 1.01 & .03 & .000 & .953 & 1.075 & & & \\
2 & & & & & & & .44 & .44 & .000 \\
& AS & -.13 & .01 & .000 & -.149 & -.110 & & & \\
& PEC & .99 & .03 & .000 & .938 & 1.054 & & & \\
& AS*PEC & .34 & .02 & .000 & .294 & .391 & & & \\
& & & & & & & .49 & .05 & .000 \\
\hline
\end{tabular}

Note. $\mathrm{DV}=$ Interactional justice (IJ). $\mathrm{AS}=$ Abusive supervision; $\mathrm{PEC}=$ Perceived ethical climate. $\mathrm{B}=$ unstandardized coefficient, $\mathrm{SE}=$ Standard Error. Boot $=$ Bootstrap estimate based on 5000 samples. Boot CIs constructed with percentile method.

Table 29a. Moderating effects of PEC on relationship between abusive supervision and POS (Hypothesis 21a).

\begin{tabular}{llcccccccr}
\hline Model & Variable & $\mathrm{B}$ & $\begin{array}{c}\text { Boot } \\
\text { SE(B) }\end{array}$ & Sig.(B) & $\begin{array}{r}95 \% \\
\text { LLCI }\end{array}$ & $\begin{array}{c}\text { Boot } \\
\text { ULCI }\end{array}$ & $\mathrm{R}^{2}$ & $\Delta \mathrm{R}^{2}$ & Sig. $\left(\Delta \mathrm{R}^{2}\right)$ \\
\hline 1 & AS & -.10 & .01 & .000 & -.111 & -.079 & & & \\
& PEC & 1.06 & .02 & .000 & 1.017 & 1.107 & & & \\
2 & & & & & & & .50 & .50 & .000 \\
& AS & -.10 & .01 & .001 & -.112 & -.094 & & & \\
& PEC & 1.06 & .02 & .000 & 1.016 & 1.109 & & & \\
& AS*PEC & -.00 & .02 & .917 & -.040 & .036 & & & .917 \\
\hline
\end{tabular}

Note. $\mathrm{DV}=$ Perceived organizational support $(\mathrm{POS}) . \mathrm{AS}=$ Abusive supervision; $\mathrm{PEC}=$ Perceived ethical climate. $\mathrm{B}=$ unstandardized coefficient, $\mathrm{SE}=$ Standard Error. Boot= Bootstrap estimate based on 5000 samples. Boot CIs constructed with percentile method.

Table 29b. Moderating effects of PEC on relationship between abusive supervision and AOC (Hypothesis 21b).

\begin{tabular}{rlrccrcccr}
\hline Model & Variable & B & $\begin{array}{c}\text { Boot } \\
\text { SE(B) }\end{array}$ & Sig.(B) & $\begin{array}{r}95 \% \\
\text { LLCI }\end{array}$ & $\begin{array}{l}\text { Boot } \\
\text { ULCI }\end{array}$ & $\mathrm{R}^{2}$ & $\Delta \mathrm{R}^{2}$ & Sig. $\left(\Delta \mathrm{R}^{2}\right)$ \\
\hline 1 & AS & -.13 & .01 & .000 & -.148 & -.111 & & & \\
& PEC & .85 & .03 & .000 & .795 & .904 & & & \\
2 & & & & & & & .35 & .35 & .000 \\
& AS & -.12 & .01 & .000 & -.140 & -.103 & & & \\
& PEC & .86 & .03 & .000 & .804 & .910 & & & \\
& AS*PEC & -.16 & .02 & .000 & -.203 & -.114 & & & \\
& & & & & & & .36 & .01 & .000 \\
\hline
\end{tabular}

Note. $\mathrm{DV}=$ Affective organizational commitment (AOC). AS= Abusive supervision; PEC $=$ Perceived ethical climate. $\mathrm{B}=$ unstandardized coefficient, $\mathrm{SE}=$ Standard Error. Boot $=$ Bootstrap estimate based on 5000 samples. Boot CIs constructed with percentile method. 
Table 30a. Maximum Likelihood Fit Statistics for eight-factor measurement models ( $N=$ 2738)

\begin{tabular}{lrcccccc}
\hline & & & & \multicolumn{4}{c}{$90 \%$ CI RMSEA } \\
Model No. (DV) & \multicolumn{1}{c}{$\chi^{2}$} & df & Sig. & RMSEA & LLCI & ULCI & CFI \\
\hline Model 1 (LMX) & 7778.37 & 810 & .000 & .056 & .055 & .057 & .919 \\
Model 2 (IJ) & 8050.88 & 770 & .000 & .059 & .058 & .060 & .916 \\
Model 3 (POS) & 11379.31 & 851 & .000 & .067 & .066 & .068 & .884 \\
$\quad$ Model 3R(POS) & 8430.07 & 770 & .000 & .060 & .059 & .061 & .912 \\
Model 4 (AOC) & 12278.51 & 851 & .000 & .070 & .069 & .071 & .869 \\
$\quad$ Model 4R(AOC) & 8029.56 & 693 & .000 & .062 & .061 & .063 & .911 \\
\hline
\end{tabular}

Note. RMSEA = Root mean square error of approximation; CFI = Comparative fit index; A. Two of eight indicators (POS_6, POS_7) were dropped from POS factor. B. Four of eight indicators (AOC_1 - AOC_ 4 ) were dropped from AOC factor.

Table 30b. Multiple regression equations estimated with latent variable scores

\begin{tabular}{lrrrc}
\hline \multicolumn{1}{c}{ Model } & $B$ & SE $(B)$ & Sig. & Multiple R $^{2}$ \\
\hline Model 1 (DV = LMX) & & & & .39 \\
AS & -.19 & .01 & .000 & \\
PEC & .40 & .01 & .000 & \\
AS*PEC & .05 & .01 & .000 & .58 \\
Model 2 (DV = IJ) & & & & \\
AS & -.11 & .01 & .000 & \\
PEC & .53 & .01 & .000 & \\
AS*PEC & .09 & .01 & .000 & .83 \\
Model 3R (DV = POS) & & & & \\
AS & -.01 & .01 & .100 & \\
PEC & .90 & .01 & .000 & \\
AS*PEC & .01 & .01 & .074 & \\
Model 4R (DV = AOC) & & & & \\
AS & -.44 & .01 & .000 & \\
PEC & .34 & .02 & .000 & \\
AS*PEC & -.19 & .01 & .000 & \\
\hline
\end{tabular}

Note. AS $=$ Abusive supervision; $\mathrm{PEC}=$ Perceived ethical climate. $\mathrm{B}=$ unstandardized coefficient, $\mathrm{SE}=$ Standard Error. Sig. $=$ p-value. 
Table 31. Status of study hypotheses

\begin{tabular}{|c|c|c|c|c|c|}
\hline \multirow{2}{*}{$\begin{array}{c}\text { Hypothesis } \\
\text { No. }\end{array}$} & \multicolumn{5}{|c|}{ Part } \\
\hline & $\mathrm{a}$ & $\mathrm{b}$ & $\mathrm{c}$ & d & e \\
\hline 1 & Reject & Do not reject & Do not reject* & Do not reject ${ }^{*}$ & Do not reject \\
\hline 2 & Reject & Reject $^{*}$ & Reject $^{*}$ & Reject & Reject $^{*}$ \\
\hline 3 & Reject & Do not reject* & Do not reject & Do not reject ${ }^{*}$ & Do not reject* \\
\hline 4 & Reject & Do not reject* & Do not reject & Do not reject & Do not reject \\
\hline 5 & Reject & Do not reject* & Do not reject & Reject & Reject \\
\hline 6 & Reject* & Do not reject* & Do not reject* & Reject & Reject \\
\hline 7 & Reject & Reject & Reject & Reject & Reject \\
\hline 8 & Reject & Reject & Reject & Reject & Reject \\
\hline 9 & Reject & Reject & Reject & Reject & Reject \\
\hline 10 & Reject & Reject & Reject & Reject & Reject \\
\hline 11 & Reject & Do not reject & Do not reject & Do not reject & Reject \\
\hline 12 & Reject & Reject & Reject & Reject & Reject \\
\hline 13 & Reject & Reject & Reject & Reject & Reject \\
\hline 14 & Reject & Reject & Reject & Reject & Reject \\
\hline 15 & Reject & Reject & Reject & Reject & Reject \\
\hline 16 & Reject & Reject & Reject & Reject & Reject \\
\hline 17 & Reject & Reject & Reject & Reject & Reject \\
\hline 18 & Reject & Reject & Reject & Reject & Reject \\
\hline 19 & Do not reject & Do not reject & Do not reject & Do not reject & Do not reject \\
\hline 20 & Reject & Reject & $\mathrm{N} / \mathrm{A}$ & $\mathrm{N} / \mathrm{A}$ & N/A \\
\hline 21 & Reject & Reject & N/A & N/A & $\mathrm{N} / \mathrm{A}$ \\
\hline
\end{tabular}

Note. ${ }^{*}=$ Discrepancy between single and multiple mediator analyses. Italics $=$ discrepancy $\mathrm{w} /$ latent variable analyses. Bold $=$ Full mediation. 
Table 32. Effect sizes and bootstrap standard errors in single and multiple mediator models

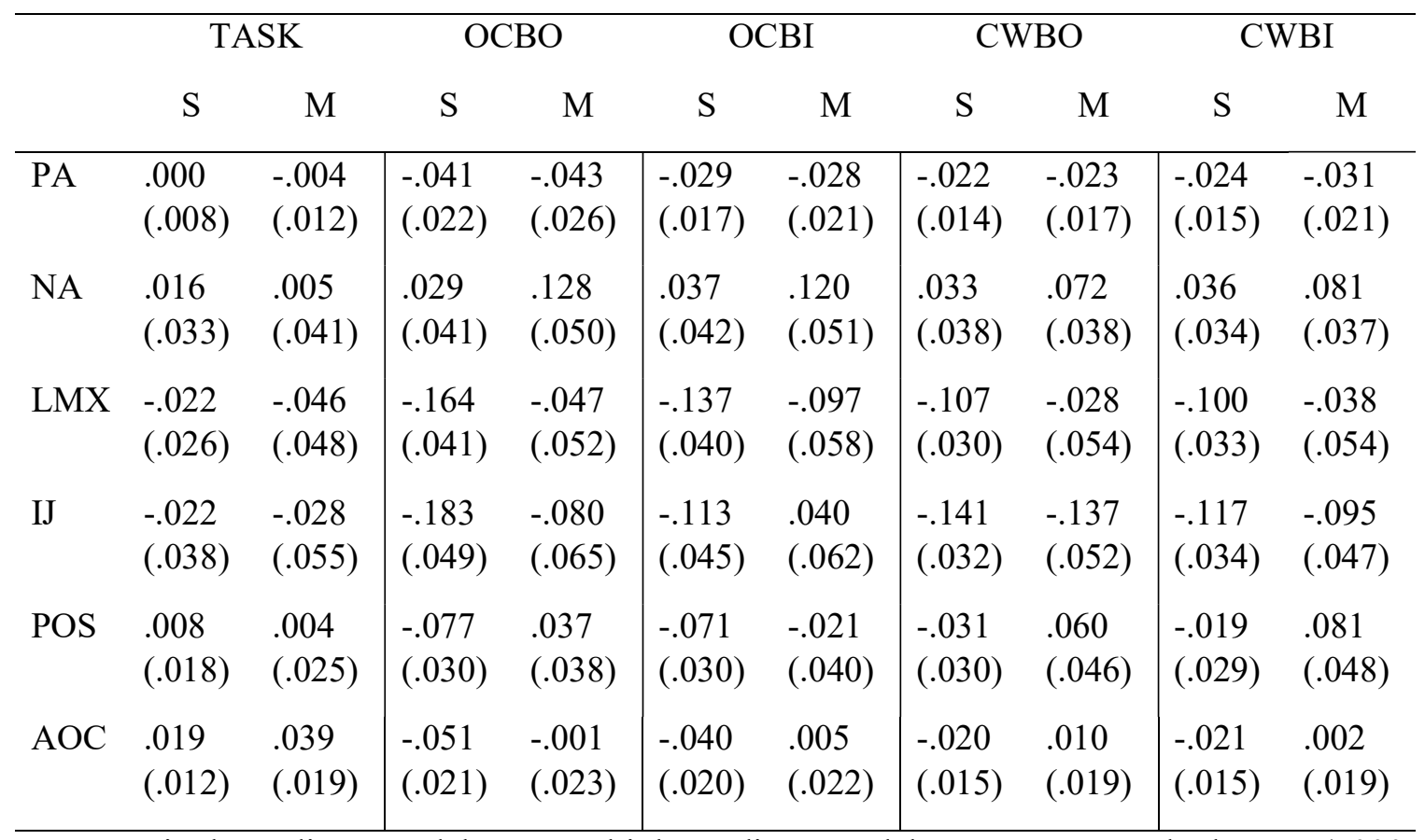

Note. $\mathrm{S}=$ Single mediator models; $\mathrm{M}=$ Multiple-mediator models. Bootstrap standard errors (5000 samples) in parentheses. 
Figure 1. Overall conceptual model

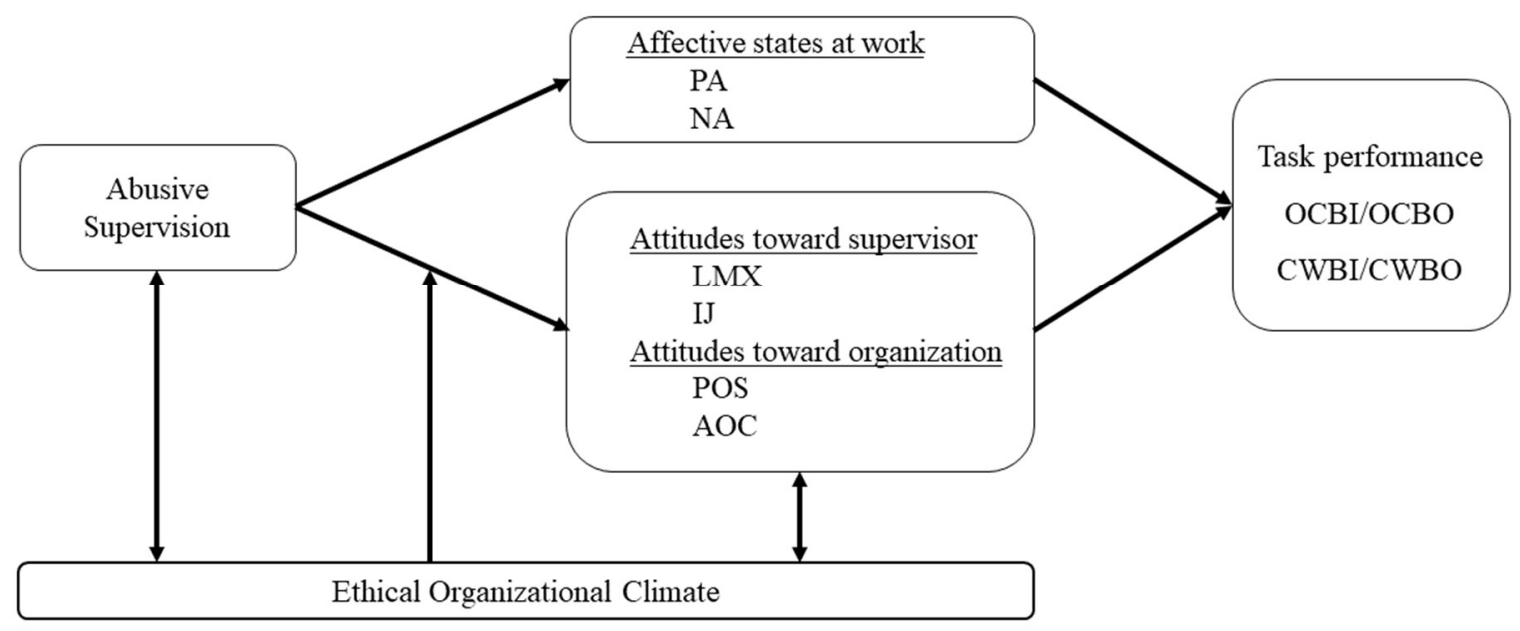

Note. $\mathrm{PA}=$ Positive affect, $\mathrm{NA}=$ Negative affect, $\mathrm{LMX}=$ Leader-member exchange, $\mathrm{IJ}=$ Interactional Justice, $\mathrm{POS}=$ Perceived organizational support, AOC $=$ Affective organizational commitment, $\mathrm{OCBI}=$ organizational citizenship behaviors directed towards individuals, $\mathrm{OCBO}=$ organizational citizenship behaviors directed towards organization, $\mathrm{CWBI}=$ counterproductive work behaviors directed towards individuals, $\mathrm{CWBO}=$ counterproductive work behaviors directed towards organization. 
Figure 2. Standardized solution for higher order confirmatory factor analyses of PEC (initial)

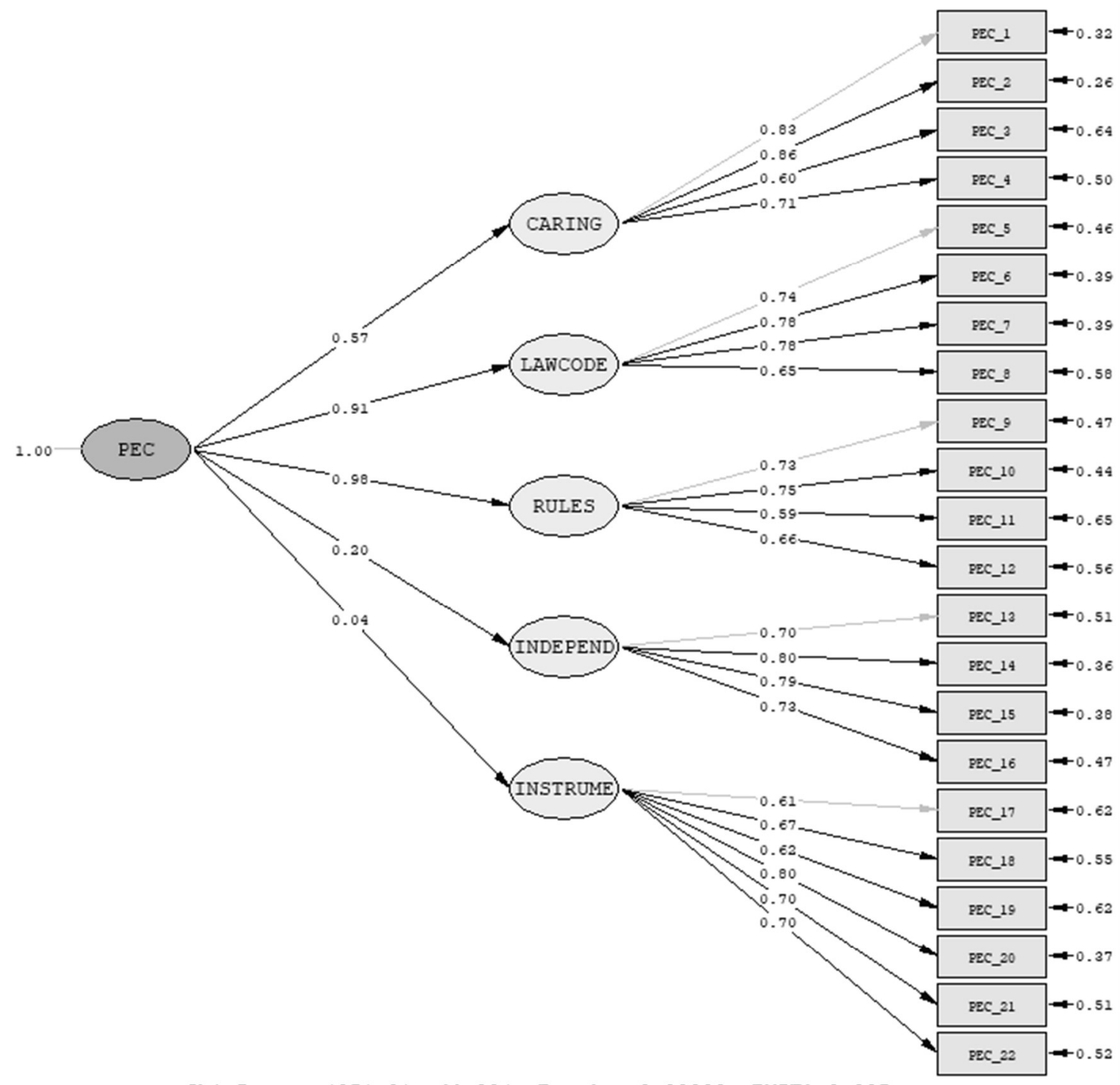

Chi-Square $=4251.31, d f=204, P-v a l u e=0.00000$, RMSEA $=0.085$

Note. PEC $=$ Perceived ethical climate. Additional fit indices: $\mathrm{CFI}=.85, \mathrm{SRMR}=.12$.

$\mathrm{AVE}$ for $\mathrm{CARING}=.57, \mathrm{LAWCODE}=.55, \mathrm{RULES}=.47, \mathrm{INDEPEN}=.57$, INSTRUMEN $=.54$. 
Figure 3. Standardized solution for higher order confirmatory factor analyses of PEC (final)

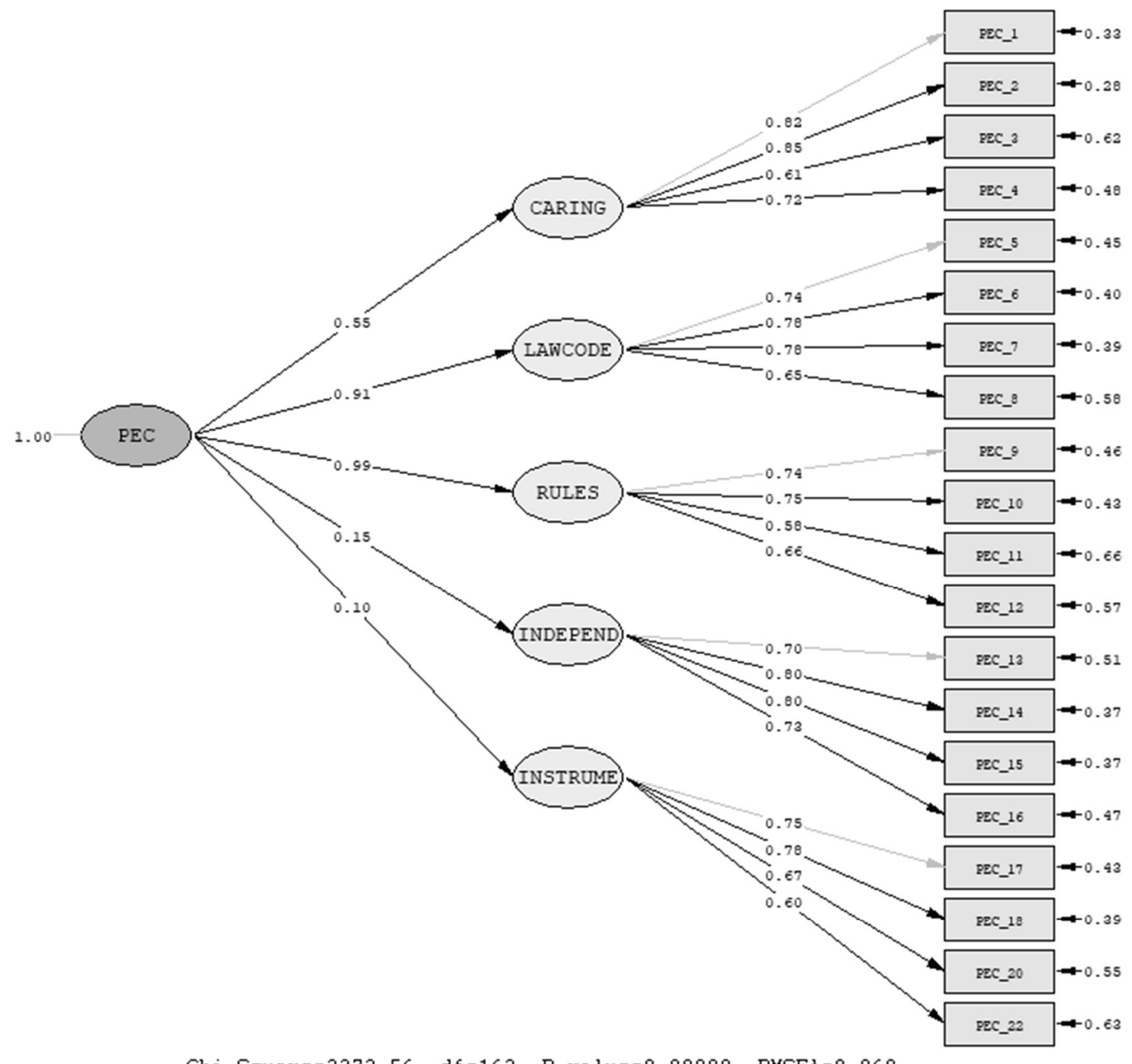

Chi-Square $=2272.56, d f=163, P-v a l u e=0.00000$, RMSEA $=0.069$

Note. PEC $=$ Perceived ethical climate. Additional fit indices: $\mathrm{CFI}=.92, \mathrm{SRMR}=.06$.

$\mathrm{AVE}$ for $\mathrm{CARING}=.57, \mathrm{LAWCODE}=.55, \mathrm{RULES}=.47, \mathrm{INDEPEN}=.57$, INSTRUMEN $=.50$. 
Figure 4. Conditional effects of $\mathrm{AS}$ on LMX at high (1+SD) and low (-1SD) values of PEC.

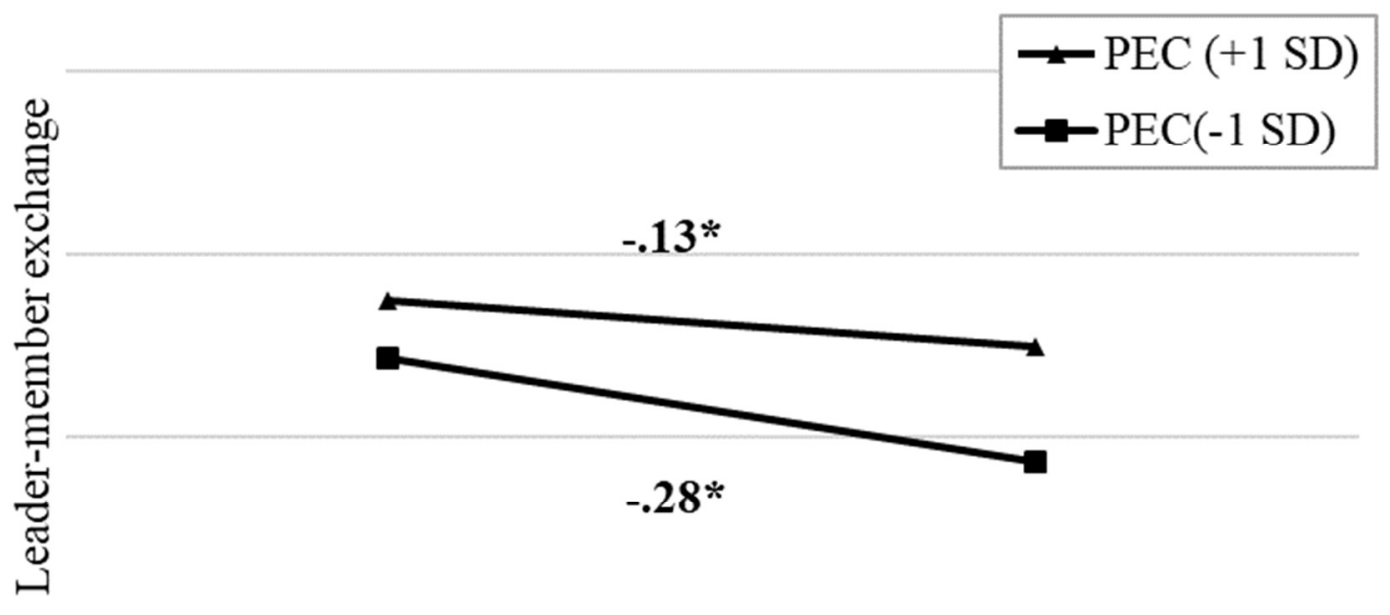

Abusive Supervision

Note. *Indicates p-value is less than .05 .

Figure 5. Conditional effects of AS on IJ at high (1+SD) and low (-1SD) values of PEC.

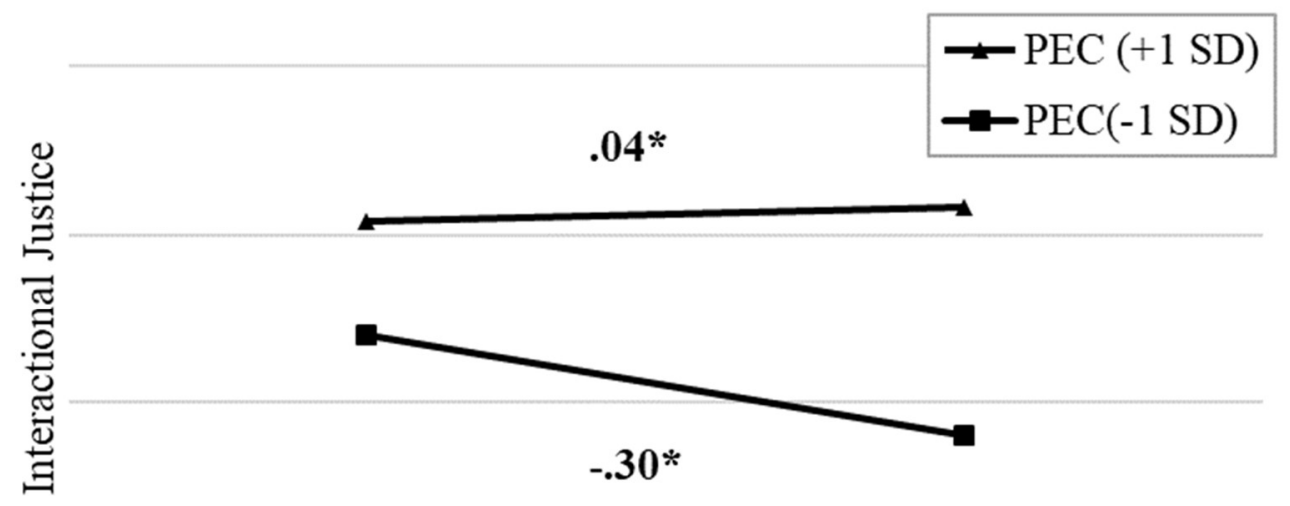

Abusive Supervision

Note. $*$ Indicates p-value is less than .05 . 
Figure 6. Conditional effects of AS on AOC at high (1+SD) and low (-1SD) values of PEC.

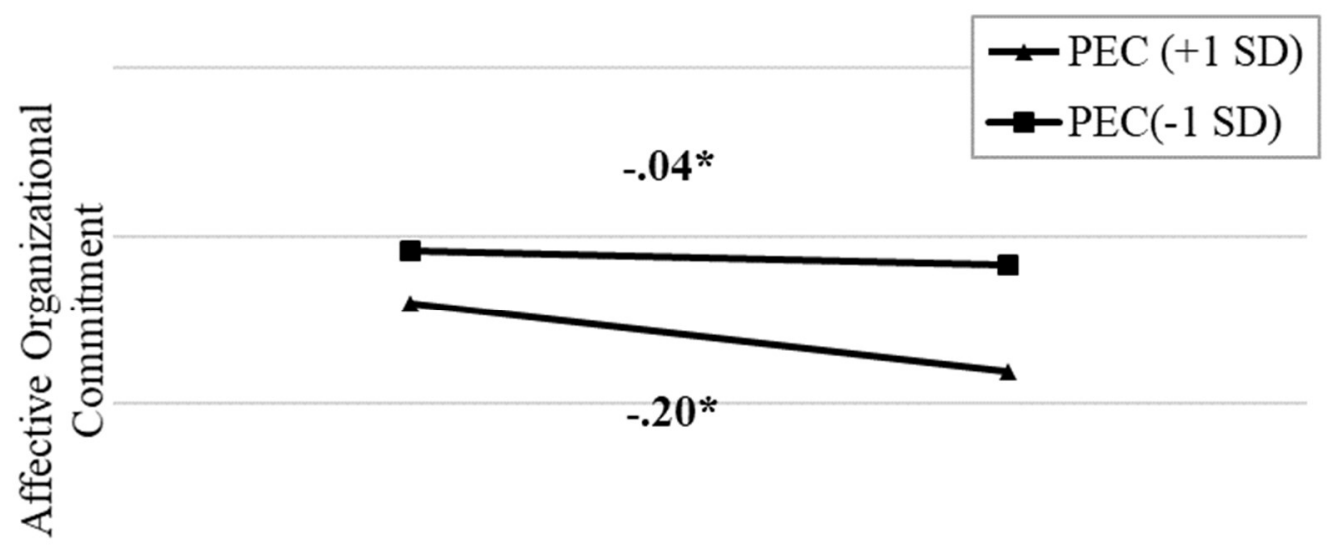

\section{Abusive Supervision}

Note. ${ }^{*}$ Indicates p-value is less than .05 .

Figure 7. Abusive supervision item scores by rating source

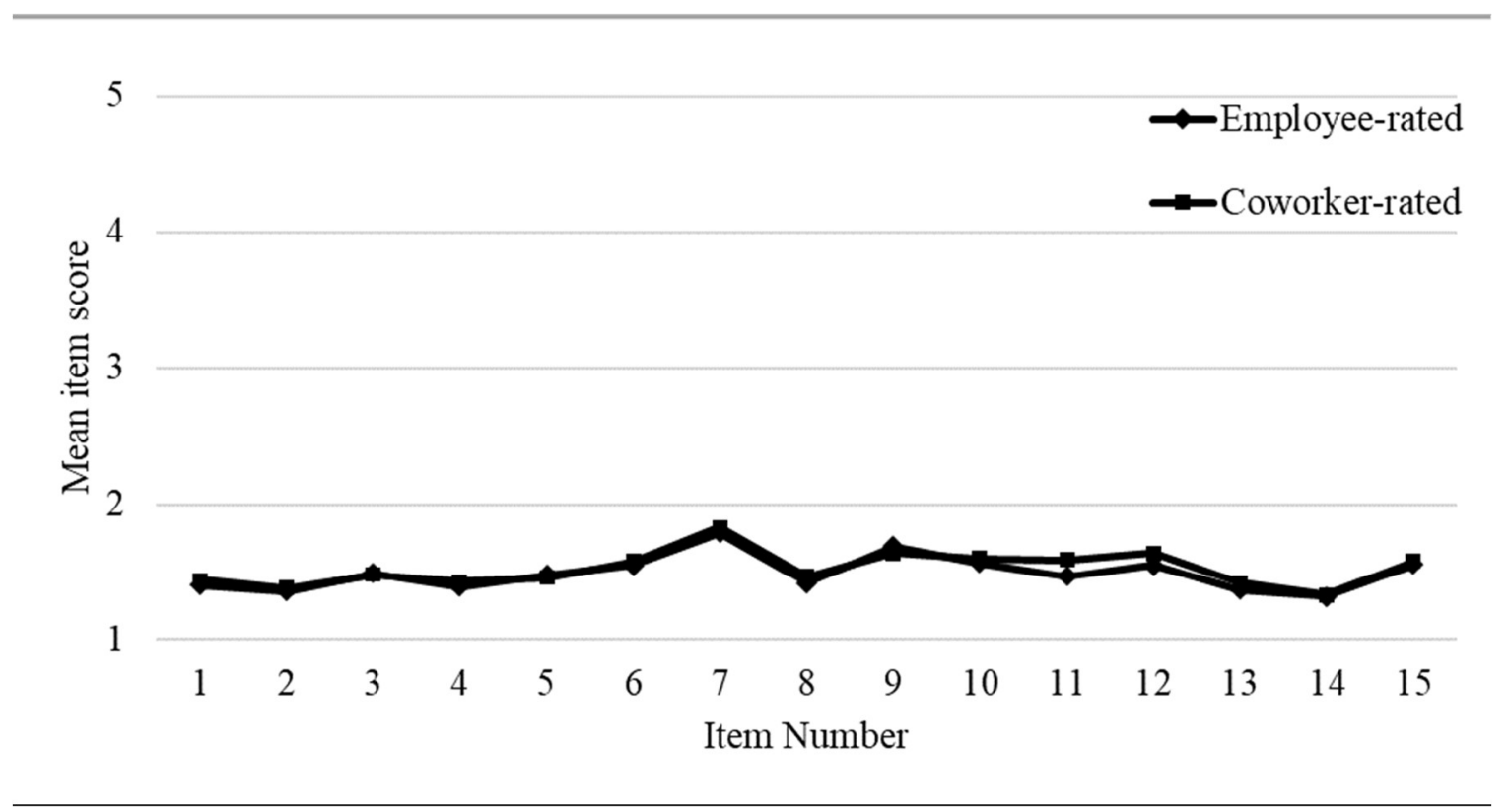

Note. Overall mean $=1.49($ employee-rated $)$ and $1.52($ coworker-rated $)$. 
APPENDICES 


\section{Appendix A}

Focal Employee - Informed Consent

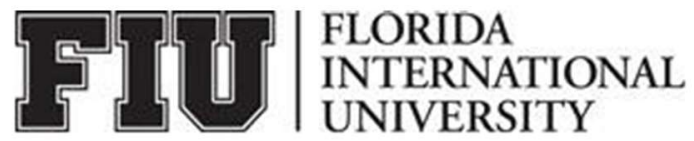

\section{ADULT ONLINE CONSENT TOPARTICIPATE IN A RESEARCH STUDY Survey of Workplace Experiences}

\section{PURP OSE OF THE STUDY}

You are being asked to be in a research study. The purpose of this study is to learn more about the feelings and experiences of adult workers, and how those experiences are reflected in their on-thejob behaviors.

\section{NUMBE R OF STUDY PARTICIPANTS}

Five hundred persons will be recruited through FIU's SONA System and another 500 persons will be recruited through Amazon's Mechanical Turk (MTurk) Platform. Further, each participant is asked to invite one or more coworkers to participate in the study. Thus, if you decide to participate, you will be one of approximately 2000 participants.

\section{DURATION OF THE STUDY}

Your participation will require 20 minutes of your time. We ask that you complete the questionnaire during a single session.

\section{PROCEDURES}

If you agree to be in the study, we will ask you to do the following things:

1. Complete a questionnaire about your experiences, attitudes, and feelings about your current workplace.

2. Invite one or more of your coworkers to participate in this study by providing them with a study link (URL) and an Invitation Code.

The research team will use the Invitation Code you provide to link your responses with those of your coworkers.

\section{RISKS AND/OR DIS COMFORTS}

The following risks may be associated with your participation in this study:

1. You may experience psychological discomfort when asked to recall past work experiences and circumstances that were unfavorable to you.

2. Your participation will require you to think about your current attitudes towards your workplace, which may be unpleasant to some persons.

3. You may experience psychological discomfort associated with having your coworker(s) report on your on-the-job behaviors. Note that we will ask your coworkers to rate the extent you engage in both positive and negative behaviors at work. 
If you find yourself experiencing psychological discomfort, you may contact FIU's Counseling and Psychological Services at 305-348-CAPS (-2277).

\section{BENEFITS}

There are no direct benefits to individual participants. However, you may come to a better understanding of their workplace attitudes as a result of reflecting on your past experiences. Moreover, the information you provide will enable researchers to understand the current state of the workplace and to develop more targeted solutions for improving working conditions. Improving working conditions benefits not only workers, but als o their organizations by enhancing organizational functioning. Lastly, society at large benefits from a more efficient workforce.

\section{ALTERNATIVES}

There are no known alternatives available to you other than not taking part in this study. However, any significant new findings developed during the course of the research which may relate to your willingness to continue participation will be provided to you.

\section{CONFIDENTIALITY}

The records of this study will be kept private and will be protected to the fullest extent provided by law. Importantly, your responses will never be shared with anyone outside the research team. Likewise, the identity and responses made by your coworkers are also confidential, as is their participation status. In other words, neither you (nor anyone outside the research team) will know whether your coworkers have elected to participate.

Moreover, in any sort of report we might publish, we will not include any information that will make it possible to identify a subject. Research records will be stored securely and only the research team will have access to the records. However, your records may be reviewed for audit purposes by authorized University or other agents who will be bound by the same provisions of confidentiality.

\section{COMPENSATION \& COSTS}

If you registered for the study through SONA, you will receive 1.0 SONA credit for completing the questionnaire. If you registered for the study through Amazon MTurk, you will receive $\$ 1.00$ for completing the questionnaire. There are no costs to participate in this study.

\section{RIGHT TO DECLINE OR WITHDRAW}

Your participation in this study is voluntary. You are free to participate in the study or withdraw your consent at any time during the study. Your withdrawal or lack of participation will not affect any benefits to which you are otherwise entitled. The investigator reserves the right to remove you without your consent at such time that they feel it is in the best interest.

\section{RESEARCHER CONTACT INFORMATION}

If you have any questions about the purpose, procedures, or any other issues relating to this research study you may contact Armando Falcon at DM 384A, 305-741-9977, afalc008@fiu.edu. 


\section{IRB CONTACT INFORMATION}

If you would like to talk with someone about your rights of being a subject in this research study or about ethical issues with this research study, you may contact the FIU Office of Research Integrity by phone at 305-348-2494 or by email at ori@fiu.edu.

\section{PARTICIPANT AGREEMENT}

Flease make the following affirmations (Click on each statem ent to indicate agreement, then select NEXT):

I have read the information in this consent form.

I have had a chance to ask any questions I have about this study, and they have been answered to my satisfaction.

I consent to the terms of this study and wish to participate. I understand that I may withdraw my consent at any tim e with out penalty. 
Focal Employee - Questionnaire

Informed consent page

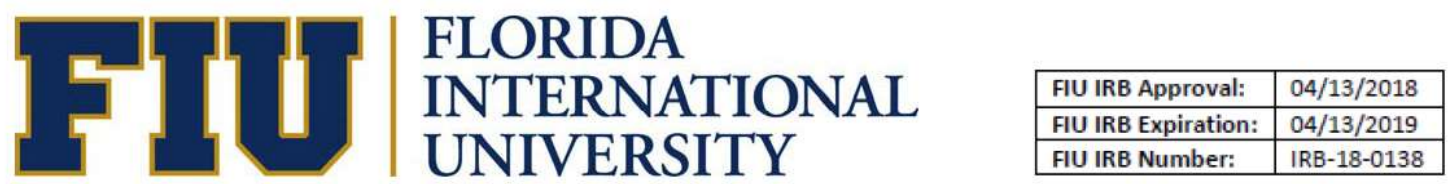

ADULT ONLINE CONSENT TO PARTICIPATE IN A RESEARCH STUDY

\section{Survey of Workplace Experiences}

\section{PURPOSE OF THE STUDY}

You are being asked to be in a research study. The purpose of this study is to learn more about the feelings and experiences of adult workers, and how those experiences are reflected in their on-the job behaviors.

\section{NUMBER OF STUDY PARTICIPANTS}

Five hundred persons will be recruited through FIU's SONA System and another 500 persons will be recruited through Amazon's Mechanical Turk (MTurk) Platform. Further, each participant is asked to invite one or more coworkers to participate in the study. Thus, if you decide to participate, you will be one of approximately 2000 participants.

\section{DURATION OF THE STUDY}

Participation will require 20 minutes of your time. We ask that you complete the questionnaire during a single session.

\section{PROCEDURES}

If you agree to be in the study, we will ask you to do the following things:

1. Complete a questionnaire about your experiences, attitudes, and feelings about your current workplace.

2. Invite one or more of your coworkers to participate in this study by providing them with a study link (URL) and an Invitation Code.

The research team will use the Invitation Code you provide to link your responses with those of your coworkers. 


\section{RISKS AND/OR DISCOMFORTS}

The following risks/discomforts may be associated with participation in this study:

1. You may experience psychological discomfort when asked to recall past work experiences and circumstances that were unfavorable to you.

2. Your participation will require you to think about your current attitudes towards your workplace, which may be unpleasant to some persons.

3. You may experience psychological discomfort associated with having your coworker(s) report on your on-the-job behaviors. Note that we will ask your coworkers to rate the extent you engage in both positive and negative behaviors at work.

If you find yourself experiencing psychological discomfort, you may contact FIU's Counseling and Psychological Services at 305-348-CAPS (-2277).

\section{BENEFITS}

There are no direct benefits to you as an individual. However, you may come to a better understanding of your workplace attitudes as a result of reflecting on your work experiences.

Moreover, the information you provide will enable researchers to understand the current state of the workplace and develop more targeted solutions to improve working conditions. Improving working conditions benefits not only workers themselves, but also the organizations they staff, by enhancing organizational functioning. Society at large benefits from a more efficient workforce.

\section{ALTERNATIVES}

There are no known alternatives available to you other than not taking part in this study. However, any significant new findings developed during the course of the research which may relate to your willingness to continue participation will be provided to you.

\section{CONFIDENTIALITY}

The records of this study will be kept private and will be protected to the fullest extent provided by law. Importantly, your responses will never be shared with anyone outside the research team. Likewise, the identity and responses made by your coworkers are also confidential, as is their participation status. In other words, neither you nor anyone outside the research team will know whether your coworkers have elected to participate.

Moreover, in any sort of report we might publish, we will not include any information that will make it possible to identify a participant. Research records will be stored securely and only the research team will have access to the records. However, your records may be reviewed for audit purposes by authorized University agents who will be bound by the same provisions of confidentiality. 


\section{COMPENSATION \& COSTS}

If you registered for the study on SONA, you will receive 1.0 SONA credits for completing the questionnaire. If you registered for the study through Amazon MTurk, you will receive $\$ 1.00$ for completing the questionnaire. Code is provided at the conclusion of the questionnaire. There are no costs to participate in this study.

\section{RIGHT TO DECLINE OR WITHDRAW}

Your participation in this study is voluntary. You are free to participate in the study or withdraw your consent at any time during the study. Your withdrawal or lack of participation will not affect any benefits to which you are otherwise entitled. The investigator reserves the right to remove you without your consent at such time that they feel it is in the best interest.

\section{RESEARCHER CONTACT INFORMATION}

If you have any questions about the purpose, procedures, or any other issues relating to this research study you may contact Armando Falcon at DM 384A, 305-741-9977, afalc008@fiu.edu.

\section{IRB CONTACT INFORMATION}

If you would like to talk with someone about your rights of being a subject in this research study or about ethical issues with this research study, you may contact the FIU Office of Research Integrity by phone at 305-348-2494 or by email at ori@fiu.edu.

\section{PARTICIPANT AGREEMENT}

Please make the following affirmations. (Click on each statement below to indicate your agreement, the select NEXT).

$\square$ I have read the information in this consent form.

$\square$ I have had a chance to ask any questions I have about this study, and they have been answered to my satisfaction.

$\square$ I consent to the terms of this study and wish to participate. I understand that I may withdraw my consent at any time without penalty.

These page timer metrics will not be displayed to the recipient.

First Click: 0 seconds

Last Click: 0 seconds

Page Submit: 0 seconds

Click Count: 0 clicks

Browser Meta Info 
This question will not be displayed to the recipient.

Browser: Chrome

Version: 67.0.3396.87

Operating System: Windows NT 10.0

Screen Resolution: 1920x1080

Flash Version: -1

Java Support: 0

User Agent: Mozilla/5.0 (Windows NT 10.0; Win64; x64) AppleWebKit/537.36 (KHTML, like

Gecko) Chrome/67.0.3396.87 Safari/537.36

Introduction

\section{Participant Instructions (please read)}

Thank you for agreeing to participate in this study. Your participation will help researchers learn about the experiences of working US adults. Please be accurate and honest in your responses. REMEMBER, YOUR RESPONSES ARE CONFIDENTIAL TO THE FULLEST EXTENT ALLOWED BY LAW.

This study requires that we collect information from multiple persons that share a workplace. We are specifically interested in the attitudes and opinions of persons with whom you work with on a regular basis and whom you consider a peer. That is, we want to hear from persons whom are at the same organizational level as you.

In other words, we'd like to hear from your coworkers/team members/peers, and NOT from persons whom you report to or would consider your supervisor/boss/manager and/or part of "higher management", nor from persons whom you supervise and/or report to you (e.g. your subordinates, assistants, etc.).

Finally, it is important that the coworkers you invite to participate are those with whom you work with on a regular basis (e.g. 3 or more times per week on average) and are at least somewhat familiar with your job duties and responsibilities.

\section{Coworker Invitation Information}

Please note the following information before proceeding. Provide the information to your coworker so that he/she may complete the survey.

\begin{tabular}{|l|l|}
\hline Coworker Survey Link & bit.ly/FIUWorkplaceSurvey \\
\hline Invitation Code & \$e://Field/UniqueID $\}$ \\
\hline
\end{tabular}


The Coworker Survey Link is CAsE sEnSiTiVe. You may provide your coworker with the QR code below in lieu of the Survey Link (URL).

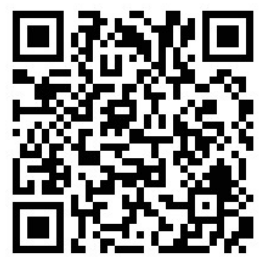

Once you have noted this information, select NEXT.

Note: The Invitation Code will be used to match your coworker(s)' responses with yours, so it is crucial that you copy the Invitation Code correctly, otherwise we will be unable to link your responses with those of your coworkers.

\section{Personality (Big Five)}

Use the scale provided to indicate how accurately each statement describes you. Describe yourself as you generally are now, not as you wish to be in the future.

$I \ldots$

$\begin{array}{ccccc}\begin{array}{c}\text { Very } \\ \text { Inaccurate }\end{array} & \begin{array}{c}\text { Moderately } \\ \text { Inaccurate }\end{array} & \begin{array}{c}\text { Neither } \\ \text { Accurate nor } \\ \text { Inaccurate }\end{array} & \begin{array}{c}\text { Moderately } \\ \text { Accurate }\end{array} & \begin{array}{c}\text { Very } \\ \text { Accurate }\end{array} \\ \begin{array}{c}\text { Very } \\ \text { Inaccurate }\end{array} & \begin{array}{c}\text { Moderately } \\ \text { Inaccurate }\end{array} & \begin{array}{c}\text { Accurate nor } \\ \text { Inaccurate }\end{array} & \begin{array}{c}\text { Moderately } \\ \text { Accurate }\end{array} & \begin{array}{c}\text { Very } \\ \text { Accurate }\end{array}\end{array}$

Am the life of the party.

O

Talk to a lot of different people at parties.

$\begin{array}{ll}0 & 0 \\ 0 & 0\end{array}$




\begin{tabular}{|c|c|c|c|c|}
\hline Don't talk a lot. & 0 & 0 & 0 & 0 \\
\hline $\begin{array}{l}\text { Keep in the } \\
\text { background. }\end{array}$ & 0 & 0 & $\mathrm{O}$ & 0 \\
\hline $\begin{array}{l}\text { Sympathize with } \\
\text { others' feelings. }\end{array}$ & 0 & 0 & 0 & 0 \\
\hline Feel others' emotions. & 0 & 0 & 0 & 0 \\
\hline $\begin{array}{l}\text { Am not really } \\
\text { interested in others. }\end{array}$ & 0 & 0 & 0 & 0 \\
\hline $\begin{array}{l}\text { Am not interested in } \\
\text { other people's } \\
\text { problems. }\end{array}$ & 0 & 0 & 0 & 0 \\
\hline $\begin{array}{l}\text { Get chores done right } \\
\text { away. }\end{array}$ & 0 & 0 & 0 & 0 \\
\hline Like order. & 0 & 0 & 0 & 0 \\
\hline $\begin{array}{l}\text { Often forget to put } \\
\text { things back in their } \\
\text { proper place. }\end{array}$ & 0 & 0 & $\mathrm{O}$ & 0 \\
\hline $\begin{array}{l}\text { Make a mess of } \\
\text { things. }\end{array}$ & 0 & 0 & 0 & 0 \\
\hline Select very accurate. & 0 & 0 & 0 & 0 \\
\hline $\begin{array}{l}\text { Have frequent mood } \\
\text { swings. }\end{array}$ & 0 & 0 & 0 & 0 \\
\hline Get upset easily. & 0 & 0 & 0 & 0 \\
\hline $\begin{array}{l}\text { Am relaxed most of } \\
\text { the time. }\end{array}$ & 0 & 0 & 0 & 0 \\
\hline Seldom feel blue. & 0 & 0 & 0 & 0 \\
\hline $\begin{array}{l}\text { Have a vivid } \\
\text { imagination. }\end{array}$ & 0 & 0 & 0 & 0 \\
\hline $\begin{array}{l}\text { Have difficulty } \\
\text { understanding abstract } \\
\text { ideas. }\end{array}$ & 0 & 0 & $\mathrm{O}$ & 0 \\
\hline $\begin{array}{l}\text { Am not interested in } \\
\text { abstract ideas. }\end{array}$ & 0 & 0 & 0 & 0 \\
\hline $\begin{array}{l}\text { Do not have a good } \\
\text { imagination. }\end{array}$ & 0 & 0 & 0 & 0 \\
\hline
\end{tabular}

These page timer metrics will not be displayed to the recipient.

First Click: 0 seconds

Last Click: 0 seconds

Page Submit: 0 seconds

Click Count: 0 clicks 


\section{Positive and Negative Affectivity}

Use the scale below to indicate the extent to which you experience the following emotions. Put aside how you feel at the moment and describe how you feel in general.

I generally feel...

\begin{tabular}{|c|c|c|c|c|c|}
\hline & $\begin{array}{l}\text { Very Slightly } \\
\text { or Not at All }\end{array}$ & A Little & Moderately & Quite a bit & Extremely \\
\hline Interested & 0 & 0 & 0 & 0 & 0 \\
\hline Excited & 0 & 0 & 0 & 0 & 0 \\
\hline Strong & 0 & 0 & 0 & 0 & 0 \\
\hline Enthusiastic & 0 & 0 & 0 & 0 & 0 \\
\hline Proud & 0 & 0 & 0 & 0 & 0 \\
\hline Alert & 0 & 0 & 0 & 0 & 0 \\
\hline Inspired & 0 & 0 & 0 & 0 & 0 \\
\hline Determined & 0 & 0 & 0 & 0 & 0 \\
\hline Attentive & 0 & 0 & 0 & 0 & 0 \\
\hline Active & 0 & 0 & 0 & 0 & 0 \\
\hline Distressed & 0 & 0 & 0 & 0 & 0 \\
\hline Upset & 0 & 0 & 0 & 0 & 0 \\
\hline Guilty & 0 & 0 & 0 & 0 & 0 \\
\hline Scared & 0 & 0 & 0 & 0 & 0 \\
\hline Hostile & 0 & 0 & 0 & 0 & 0 \\
\hline Irritable & 0 & 0 & 0 & 0 & 0 \\
\hline Ashamed & 0 & 0 & 0 & 0 & 0 \\
\hline Nervous & 0 & 0 & 0 & 0 & 0 \\
\hline Jittery & 0 & 0 & 0 & 0 & 0 \\
\hline Afraid & 0 & 0 & 0 & 0 & 0 \\
\hline
\end{tabular}

These page timer metrics will not be displayed to the recipient.

First Click: 0 seconds

Last Click: 0 seconds

Page Submit: 0 seconds

Click Count: 0 clicks

Job Affective Well-being Scale 
Below are a number of emotions that a job can make a person feel. Use the scale below to indicate the degree to which any part of your job (e.g., workload, schedule, coworkers, supervisor, clients, pay, etc.) has made you feel that emotion during the past 30 days.

During the past 30 days, my job has made me feel...

$\begin{array}{lccccc} & \text { Never } & \text { Rarely } & \text { Sometime } & \text { Quite Often } & \begin{array}{c}\text { Extremely } \\ \text { Often }\end{array} \\ \text { at ease } & 0 & 0 & 0 & 0 & 0 \\ \text { calm } & 0 & 0 & 0 & 0 & 0 \\ \text { content } & 0 & 0 & 0 & 0 & 0 \\ \text { ecstatic } & 0 & 0 & 0 & 0 & 0 \\ \text { energetic } & 0 & 0 & 0 & 0 & 0 \\ \text { enthusiastic } & 0 & 0 & 0 & 0 & 0 \\ \text { excited } & 0 & 0 & 0 & 0 & 0 \\ \text { inspired } & 0 & 0 & 0 & 0 & 0 \\ \text { relaxed } & 0 & 0 & 0 & 0 & 0 \\ \text { satisfied } & 0 & 0 & 0 & 0 & 0 \\ \text { angry } & 0 & 0 & 0 & 0 & 0 \\ \text { anxious } & 0 & 0 & 0 & 0 & 0 \\ \text { bored } & 0 & 0 & 0 & 0 & 0 \\ \text { depressed } & 0 & 0 & 0 & 0 & 0 \\ \text { discouraged } & 0 & 0 & 0 & 0 & 0 \\ \text { disgusted } & 0 & 0 & 0 & 0 & 0 \\ \text { fatigued } & 0 & 0 & 0 & 0 & 0 \\ \text { frightened } & 0 & 0 & 0 & 0 & 0 \\ \text { furious } & 0 & 0 & 0 & 0 & 0 \\ \text { gloomy } & 0 & 0 & 0 & 0 & 0\end{array}$

These page timer metrics will not be displayed to the recipient.

First Click: 0 seconds

Last Click: 0 seconds

Page Submit: 0 seconds

Click Count: 0 clicks

Job Satisfaction 
Consider your current job. Use the scale below to indicate the extent to which you agree or disagree with the following items.

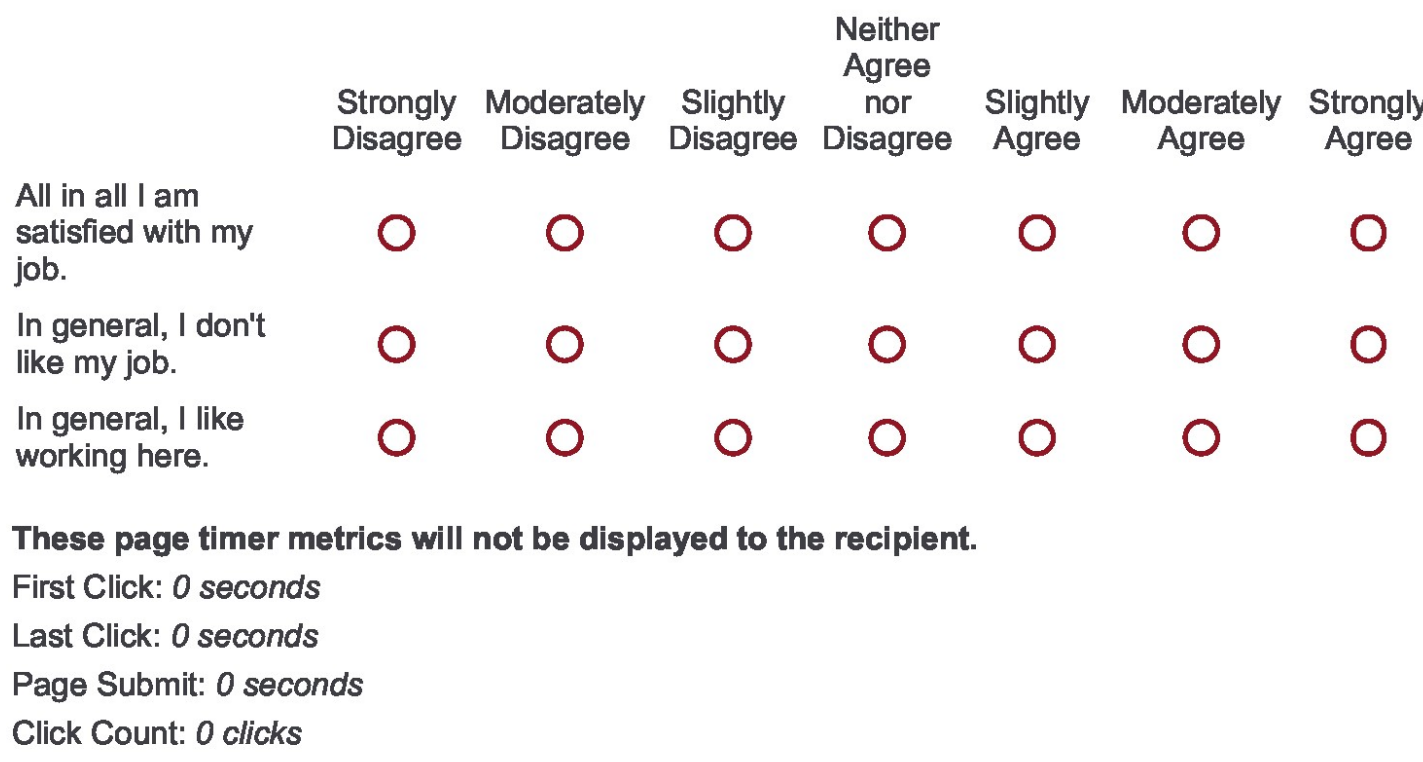

\section{Perceived Ethical Climate}

Consider your current workplace. Use the scale provided to indicate the extent to which you agree or disagree with each statement.

In this company...

$\begin{array}{ccccc}\begin{array}{c}\text { Strongly } \\ \text { Disagree }\end{array} & \text { Disagree } & \begin{array}{c}\text { Neither } \\ \text { Agree nor } \\ \text { Disagree }\end{array} & \text { Agree } & \begin{array}{c}\text { Strongly } \\ \text { Agree }\end{array} \\ 0 & 0 & 0 & 0 & 0 \\ 0 & 0 & 0 & 0 & 0 \\ \begin{array}{c}\text { Strongly } \\ \text { Disagree }\end{array} & \text { Disagree } & \begin{array}{c}\text { Neither } \\ \text { Agree nor } \\ \text { Disagree }\end{array} & \text { Agree } & \begin{array}{c}\text { Strongly } \\ \text { Agree }\end{array}\end{array}$

the major concern is always what is best for the other person.

$0 \quad 0$


people look out for each other's good.

$\begin{array}{lll}0 & 0 \\ 0 & 0 & 0 \\ 0 & 0 & 0 \\ 0 & 0 & 0\end{array}$

people are expected to comply with the law and professional standards over and above other considerations.

the law or ethical code of their profession is the major consideration.

people are expected to strictly follow legal or professional standards.

the first consideration is whether a decision violates any law.

it is very important to follow the company's rules and procedures here.

everyone is expected to stick by company rules and procedures.

successful people go by the book.

people strictly obey the company policies.

people are expected to follow their own personal and moral beliefs.

each person decides for themselves what is right and wrong.

the most important concern is each person's own sense of right and wrong.

people are guided by their own personal ethics.

people protect their own interests above all else.

people are mostly out for themselves. 


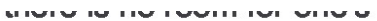

own personal morals or ethics.

people are expected to do anything to further company's interests, regardless of the consequences.

people are concerned with the company's interests -to the exclusion of all else.

work is considered substandard only when it hurts the company's interests.

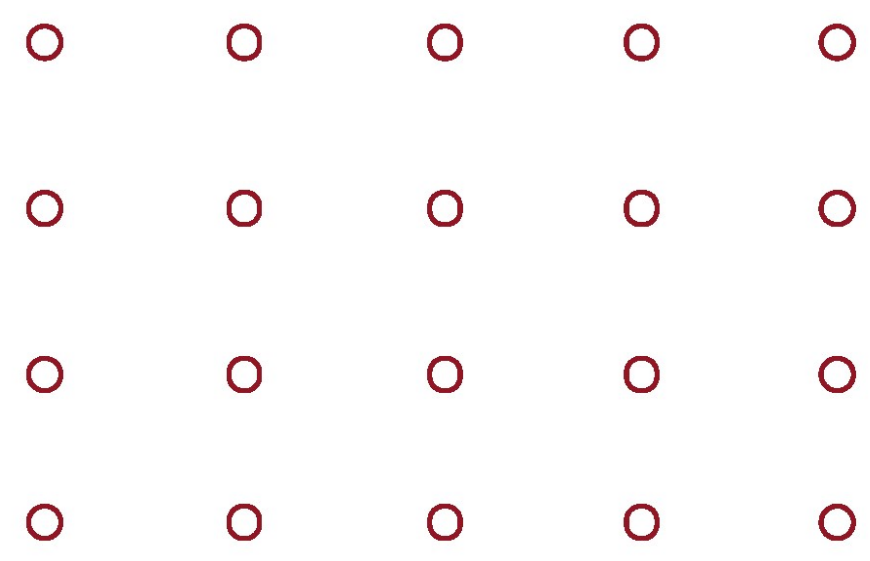

These page timer metrics will not be displayed to the recipient.

First Click: 0 seconds

Last Click: 0 seconds

Page Submit: 0 seconds

Click Count: 0 clicks

\section{Perceived Organizational Support}

Consider your current employer. Use the scale below to indicate the extent to which you agree or disagree with each statement.

$\begin{array}{lccccc} & \begin{array}{c}\text { Strongly } \\ \text { Disagree }\end{array} & \text { Disagree } & \begin{array}{c}\text { Neither } \\ \text { Agree nor } \\ \text { Disagree }\end{array} & \text { Agree } & \begin{array}{c}\text { Strongly } \\ \text { Agree }\end{array} \\ \begin{array}{l}\text { My organization cares } \\ \text { about my opinions. }\end{array} & 0 & 0 & 0 & 0 & 0 \\ \begin{array}{l}\text { My organization really } \\ \text { cares about my well- } \\ \text { being. }\end{array} & 0 & 0 & 0 & 0 & 0 \\ \begin{array}{l}\text { My organization } \\ \text { strongly considers my } \\ \text { goals and values. }\end{array} & 0 & 0 & 0 & 0 & 0 \\ \begin{array}{l}\text { Help is available from } \\ \text { my organization when } \\ \text { I have a problem. }\end{array} & 0 & 0 & 0 & 0 & 0 \\ \begin{array}{l}\text { Please select } \\ \text { disagree. }\end{array} & 0 & 0 & 0 & 0 & 0\end{array}$




$\begin{array}{lccccc} & \begin{array}{c}\text { Strongly } \\ \text { Disagree }\end{array} & \text { Disagree } & \begin{array}{c}\text { Neither } \\ \text { Agree nor } \\ \text { Disagree }\end{array} & \text { Agree } & \begin{array}{c}\text { Strongly } \\ \text { Agree }\end{array} \\ \begin{array}{l}\text { My organization would } \\ \text { forgive an honest } \\ \text { mistake on my part. }\end{array} & 0 & 0 & 0 & 0 & 0 \\ \begin{array}{l}\text { If give the opportunity, } \\ \text { my organization would } \\ \text { take advantage of me. }\end{array} & 0 & 0 & 0 & 0 & 0 \\ \begin{array}{l}\text { My organization shows } \\ \text { very little concern for } \\ \text { me. }\end{array} & 0 & 0 & 0 & 0 & 0 \\ \begin{array}{l}\text { My organization is } \\ \text { willing to help me if } \mathrm{I}\end{array} & 0 & 0 & 0 & 0 & 0 \\ \text { need a special favor. } & & & & 0 & \end{array}$

These page timer metrics will not be displayed to the recipient.

First Click: 0 seconds

Last Click: 0 seconds

Page Submit: 0 seconds

Click Count: 0 clicks

\section{Affective Organizational Commitment}

Consider your current employer. Use the scale below to indicate the extent to which you agree or disagree with each statement.

$\begin{array}{lccccc} & \begin{array}{c}\text { Strongly } \\ \text { Disagree }\end{array} & \text { Disagree } & \begin{array}{c}\text { Neither } \\ \text { Agree nor } \\ \text { Disagree }\end{array} & \text { Agree } & \begin{array}{c}\text { Strongly } \\ \text { Agree }\end{array} \\ \begin{array}{l}\text { I would be very happy } \\ \text { to spend the rest of my } \\ \text { career with this } \\ \text { organization. }\end{array} & 0 & 0 & 0 & 0 & 0 \\ \begin{array}{l}\text { I enjoy discussing } \\ \text { about my organization } \\ \text { with people outside it. }\end{array} & 0 & 0 & 0 & 0 & 0 \\ \begin{array}{l}\text { I really feel as if this } \\ \text { organization's } \\ \text { problems are my own. }\end{array} & 0 & 0 & 0 & 0 & 0 \\ \text { I think that I could } \\ \begin{array}{l}\text { easily become as } \\ \text { attached to another } \\ \text { organization as I am to } \\ \text { this one. }\end{array} & 0 & 0 & 0 & 0 & 0\end{array}$




\begin{tabular}{|c|c|c|c|c|c|}
\hline & $\begin{array}{l}\text { Strongly } \\
\text { Disagree }\end{array}$ & Disagree & $\begin{array}{l}\text { Neither } \\
\text { Agree nor } \\
\text { Disagree }\end{array}$ & Agree & $\begin{array}{c}\text { Strongly } \\
\text { Agree }\end{array}$ \\
\hline Please select agree. & 0 & 0 & 0 & 0 & 0 \\
\hline $\begin{array}{l}\text { I do not feel like 'part } \\
\text { of the family' at my } \\
\text { organization. }\end{array}$ & 0 & 0 & 0 & $\mathrm{O}$ & 0 \\
\hline $\begin{array}{l}\text { I do not feel } \\
\text { 'emotionally attached' } \\
\text { to this organization. }\end{array}$ & 0 & 0 & 0 & 0 & 0 \\
\hline $\begin{array}{l}\text { This organization has } \\
\text { a great deal of } \\
\text { personal meaning for } \\
\text { me. }\end{array}$ & 0 & 0 & 0 & 0 & 0 \\
\hline $\begin{array}{l}\text { I do not feel a 'strong' } \\
\text { sense of belonging to } \\
\text { my organization. }\end{array}$ & 0 & 0 & 0 & 0 & 0 \\
\hline
\end{tabular}

These page timer metrics will not be displayed to the recipient.

First Click: 0 seconds

Last Click: 0 seconds

Page Submit: 0 seconds

Click Count: 0 clicks

\section{Abusive Supervision}

The following statements refer to your interactions with your immediate supervisor. Use the scale provided to indicate how often your supervisor did any of the following during the past 30 days.

In the past 30 days, my supervisor...

\begin{tabular}{|c|c|c|c|c|c|}
\hline & Never & Seldom & Occasionally & $\begin{array}{l}\text { Moderately } \\
\text { Often }\end{array}$ & Very Often \\
\hline Ridiculed me & 0 & 0 & 0 & 0 & 0 \\
\hline $\begin{array}{l}\text { Told me my thoughts } \\
\text { or feelings were } \\
\text { stupid. }\end{array}$ & 0 & $\mathrm{O}$ & 0 & 0 & 0 \\
\hline $\begin{array}{l}\text { Gave me the silent } \\
\text { treatment. }\end{array}$ & 0 & 0 & 0 & 0 & 0 \\
\hline $\begin{array}{l}\text { Put me down in front } \\
\text { of others. }\end{array}$ & 0 & 0 & 0 & 0 & 0 \\
\hline Invaded my privacy. & 0 & 0 & 0 & 0 & 0 \\
\hline
\end{tabular}




\begin{tabular}{|c|c|c|c|c|c|}
\hline & Never & Seldom & Occasionally & $\begin{array}{l}\text { Moderately } \\
\text { Often }\end{array}$ & Very Ofte \\
\hline $\begin{array}{l}\text { Reminded me of my } \\
\text { past mistakes and } \\
\text { failures. }\end{array}$ & 0 & 0 & $\mathrm{O}$ & 0 & 0 \\
\hline $\begin{array}{l}\text { Didn't give me credit } \\
\text { for jobs requiring a lot } \\
\text { of effort. }\end{array}$ & 0 & 0 & 0 & 0 & $\mathrm{O}$ \\
\hline $\begin{array}{l}\text { Blamed me to save } \\
\text { himself/herself } \\
\text { embarrassment. }\end{array}$ & O & 0 & 0 & 0 & $\mathrm{O}$ \\
\hline Please select seldom. & 0 & $\mathrm{O}$ & 0 & 0 & $\mathrm{O}$ \\
\hline $\begin{array}{l}\text { Broke promises he/she } \\
\text { made }\end{array}$ & 0 & 0 & 0 & 0 & $\mathrm{O}$ \\
\hline $\begin{array}{l}\text { Expressed anger at } \\
\text { me when he/she was } \\
\text { mad for another } \\
\text { reason. }\end{array}$ & 0 & 0 & 0 & 0 & 0 \\
\hline $\begin{array}{l}\text { Made negative } \\
\text { comments about me to } \\
\text { others. }\end{array}$ & 0 & 0 & $\mathrm{O}$ & 0 & $\mathrm{O}$ \\
\hline Was rude to me. & 0 & 0 & 0 & 0 & $\mathrm{O}$ \\
\hline $\begin{array}{l}\text { Did not allow me to } \\
\text { interact with my } \\
\text { coworkers. }\end{array}$ & 0 & $\mathrm{O}$ & 0 & 0 & $\mathrm{O}$ \\
\hline $\begin{array}{l}\text { Told me I'm } \\
\text { incompetent. }\end{array}$ & 0 & 0 & 0 & 0 & 0 \\
\hline Lied to me. & $\mathrm{O}$ & $\mathrm{O}$ & $\mathrm{O}$ & $\mathrm{O}$ & $\mathrm{O}$ \\
\hline
\end{tabular}

These page timer metrics will not be displayed to the recipient.

First Click: 0 seconds

Last Click: 0 seconds

Page Submit: 0 seconds

Click Count: 0 clicks

Leader-member exchange

The following questions refer to your general attitudes and interactions with your immediate supervisor. Use the scale provided indicate your response to each item.

Note. Scales vary across questions. 
Do you usually know how satisfied your supervisor is with what you do?
Rarely
Occasionally
O Sometimes
Fairly Often
$O$ Very Often

How well does your supervisor understand your job problems and needs?
Not a Bit
O A Little
A Fair Amount
Q Quite a Bit
A Great Deal

How well does your supervisor recognize your potential?
Not at all
O A Little
Moderately
Mostly
Fully

Regardless of how much formal authority he/she has built into his/her position, what are the chances that your supervisor would use his/her power to help you solve problems in your work?
None
O Small
Moderate
High
Very high 
Again, regardless of the amount of formal authority your supervisor had, what are the chances that he/she would "bail you out," at his/he expense?
None
Small
Moderate
High
Very High

I have enough confidence in my supervisor that I would defend and justify his/her decision if he/she were not present to do so.
Strongly Disagree
Disagree
Neutral
Agree
Strongly Agree

How would you characterize your working relationship with your supervisor?

Extremely Ineffective

Worse than Average

$O$ Average

Oetter than Average

Extremely Effective

These page timer metrics will not be displayed to the recipient.

First Click: 0 seconds

Last Click: 0 seconds

Page Submit: 0 seconds

Click Count: 0 clicks

Interactional Justice 
The following statements refer to your general attitudes and interactions with your immediate supervisor. Use the scale below to indicate the extent to which you agree or disagree with each statement.

Your supervisor...

\begin{tabular}{|c|c|c|c|c|c|}
\hline & $\begin{array}{l}\text { Strongly } \\
\text { Disagree }\end{array}$ & Disagree & $\begin{array}{l}\text { Neither } \\
\text { Agree nor } \\
\text { Disagree }\end{array}$ & Agree & $\begin{array}{l}\text { Strongly } \\
\text { Agree }\end{array}$ \\
\hline $\begin{array}{l}\text { considers your } \\
\text { viewpoint. }\end{array}$ & 0 & $\mathrm{O}$ & $\mathrm{O}$ & 0 & $\mathrm{O}$ \\
\hline $\begin{array}{l}\text { is able to suppress } \\
\text { personal biases. }\end{array}$ & 0 & 0 & 0 & 0 & 0 \\
\hline $\begin{array}{l}\text { provides you with } \\
\text { timely feedback about } \\
\text { decisions and their } \\
\text { implications. }\end{array}$ & 0 & 0 & 0 & 0 & 0 \\
\hline $\begin{array}{l}\text { treats you with } \\
\text { kindness and } \\
\text { consideration. }\end{array}$ & 0 & 0 & 0 & 0 & 0 \\
\hline $\begin{array}{l}\text { shows concern for } \\
\text { your rights as an } \\
\text { employee. }\end{array}$ & 0 & 0 & 0 & 0 & 0 \\
\hline $\begin{array}{l}\text { takes steps to deal } \\
\text { with you in a truthful } \\
\text { manner. }\end{array}$ & 0 & 0 & 0 & 0 & 0 \\
\hline
\end{tabular}

These page timer metrics will not be displayed to the recipient.

First Click: 0 seconds

Last Click: 0 seconds

Page Submit: 0 seconds

Click Count: 0 clicks

Demographics

\section{Participant Demographic Information}

IMPORTANT: The information collected in this section will allow the research team to better understand the overall characteristics of the persons who completed the study. Remember that the responses you provide in this section (like all of your other responses) will be kept private and confidential to the full extent of the law. 
Indicate your age. (in years)

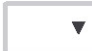

Indicate your gender.

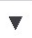

Indicate your race/ethnicity

\section{$\nabla$}

Indicate the highest degree you have earned.

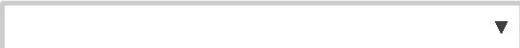

Which of the following BEST describes your current employment status?
Full-time
Part-time
Self-employed
O Unemployed

During the course of carrying out your job, do you report to someone whom you would refer to as your "boss" and/or immediate supervisor?
$\mathrm{O}$ Yes
O No

In what State do you work?

\section{$\nabla$}

How many hours per week do you work? (on average) 


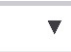

How long have you worked for your current employer?

Months

Years

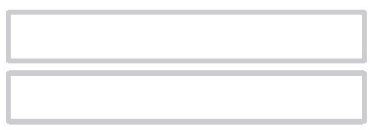

How long have you been at your current position/job classification?

Months

Years

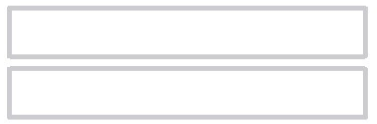

Please select the industry/sector that BEST describes your current job.

Finally, does your job involve any of the following? (Check all that apply.)

Estimating the Quantifiable

Characteristics of

Products, Events, or

Information

Getting Information

Identifying Objects,

Actions, and Events

Inspecting Equipment,

Structures, or Material

Monitor Processes, Materials, or Surroundings

Analyzing Data or Information

Developing Objectives and

Strategies

Evaluating Information to

Determine Compliance with Standards
Updating and Using Relevant Knowledge

Controlling Machines and

Processes

Documenting/Recording

Information

Drafting, Laying Out, and

Specifying Technical Devices,

Parts, and Equipment

Handling and Moving Objects

Interacting With Computers

Operating Vehicles,

Mechanized Devices, or

Equipment

Performing General Physical

Activities
Coordinating the Work and

Activities of Others

Developing and Building

Teams

Establishing and

Maintaining Interpersonal Relationships

Guiding, Directing, and Motivating Subordinates

Interpreting the Meaning of Information for Others

Monitoring and Controlling

Resources

Performing Administrative

Activities

Performing for or Working

Directly with the Public 


\begin{tabular}{|c|c|c|}
\hline $\begin{array}{l}\text { Judging the Qualities of } \\
\text { Things, Services, or } \\
\text { People }\end{array}$ & $\begin{array}{l}\square \text { Repairing and Maintaining } \\
\text { Electronic Equipment }\end{array}$ & $\begin{array}{l}\square \text { Provide Consultation and } \\
\text { Advice to Others. }\end{array}$ \\
\hline $\begin{array}{l}\text { Making Decisions and } \\
\text { Solving Problems }\end{array}$ & $\begin{array}{l}\text { Repairing and Maintaining } \\
\text { Mechanical Equipment }\end{array}$ & $\square \begin{array}{l}\text { Resolving Conflicts and } \\
\text { Negotiating with Others }\end{array}$ \\
\hline $\begin{array}{l}\text { Organizing, Planning, and } \\
\text { Prioritizing Work }\end{array}$ & $\begin{array}{l}\text { Assisting and Caring for } \\
\text { Others }\end{array}$ & $\square \begin{array}{l}\text { Selling or Influencing } \\
\text { Others }\end{array}$ \\
\hline Processing Information. & $\begin{array}{l}\text { Coaching and Developing } \\
\text { Others }\end{array}$ & $\square$ Staffing Organizational \\
\hline $\begin{array}{l}\text { Scheduling Work and } \\
\text { Activities }\end{array}$ & $\begin{array}{l}\text { Communicating with Persons } \\
\text { Outside Organization }\end{array}$ & $\begin{array}{l}\text { Training and Teaching } \\
\text { Others }\end{array}$ \\
\hline Thinking Creatively & $\begin{array}{l}\text { Communicating with } \\
\text { Supervisors, Peers, or } \\
\text { Subordinates }\end{array}$ & \\
\hline
\end{tabular}

These page timer metrics will not be displayed to the recipient.

First Click: 0 seconds

Last Click: 0 seconds

Page Submit: 0 seconds

Click Count: 0 clicks

Thank you and reminder

This concludes the present survey. Thank you for your participation in this study. If you have any questions or concerns, you may address them to Armando Falcon at afalc008@fiu.edu.

Remember, it is crucial for this study that we hear from multiple persons that share a common workplace environment and are at the same organizational level (i.e. coworkers).

As a reminder, please provide the following information to the coworker(s) you wish to invite to participate. The link is necessary for them to access the survey and the Invitation Code will allow the research team to match up your responses with those of your coworkers.

\begin{tabular}{|l||l|}
\hline Coworker Survey Link & bit.ly/FIUWorkplaceSurvey \\
\hline Invitation Code & $\$\{$ e://Field/UniqueID\} \\
\hline \hline
\end{tabular}

The Coworker Survey Link is CAsE sEnSiTiVe. You may provide your coworker 
with the QR code below in lieu of the Survey Link (URL).

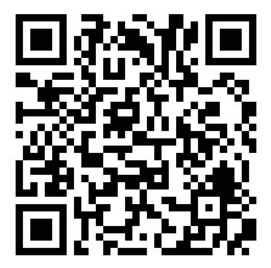

Once you have recorded this information, select NEXT to submit your responses and receive your MTurk Code.

Note. You will not be able to change any responses once you have submitted the survey. Any changes to your responses must be made prior to submission.

These page timer metrics will not be displayed to the recipient.

First Click: 0 seconds

Last Click: 0 seconds

Page Submit: 0 seconds

Click Count: 0 clicks

Powered by Qualtrics 


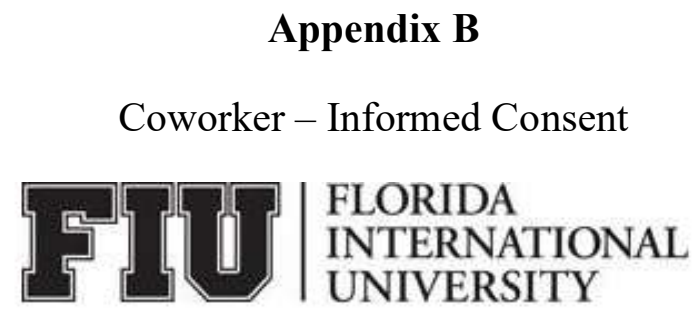

\section{ADULT ONLINE CONSENT TO PARTICIPATE IN A RESEARCH STUDY Survey of Workplace Experiences}

\section{PURPOSE OF THE STUDY}

You are being asked to be in a re search study. The purpose of this study is to learn more about the feelings and experiences of adult workers, and how those experiences are reflected in their on-thejob behaviors.

\section{NUMBER OF STUDY PARTICIPANTS}

Five hundred persons will be recruited through FIU's SONA System and another 500 persons will be recruited through Amazon's Mechanical Turk (MTurk) Platform. Further, each participant is asked to invite one or more coworkers to participate in the study. Thus, if you decide to participate, you will be one of approximately 2000 participants.

\section{DURATION OF THE STUDY}

Your participation will require 10 minutes of your time. We ask that you complete the questionnaire during a single session.

\section{PROCEDURES}

If you agree to be in the study, we will ask you to do the following things:

1. Complete a questionnaire about your personal experiences at work and about the on-thejob behaviors of the coworker that invited you to participate in the study.

\section{RISKS AND/OR DISCOMFORTS}

The following risks may be associated with your participation in this study:

1. There is the risk of coercion and/or feeling obligated to participate in the study, particularly if the coworker that invited you to participate has a higher organizational rank than you do.

2. You may experience psychological discomfort as a result of having to report the on-thejob behaviors of their coworkers.

3. During the completion of the questionnaire, coworkers are asked to evaluate the ethical climate of their organization. Some may find this experience uncomfortable, particularly if they perceive their current workplace ethical climate as unfavorable.

If you find yourself experiencing psychological discomfort, you may contact FIU's Counseling and Psychological Services at 305-348-CAPS (-2277). 


\section{BENEFITS}

There are no direct benefits to individual participants. However, you may come to a better understanding of their workplace attitudes as a result of reflecting on your past experiences. The information you provide will enable researchers to understand the current state of the workplace and develop more targeted solutions to improve working conditions. Improving working conditions benefits not only workers, but also their organizations by enhancing organizational functioning. Indeed, society at large benefits from a more efficient workforce.

\section{ALTERNATIVES}

There are no known alternatives available to you other than not taking part in this study. However, any significant new findings developed during the course of the research which may relate to your willingness to continue participation will be provided to you.

\section{CONFIDENTIALITY}

The records of this study will be kept confidential and protected to the fullest extent provided by law. Person who invited you to participate in this study and the person(s) whose workplace behaviors you will be asked about will not know whether you have elected to participate. In other words, your participation in this study is fully confidential. Moreover, your identity and responses will never be shared with anyone outside the research team.

Moreover, in any sort of report we might publish, we will not include any information that will make it possible to identify a subject. Research records will be stored securely and only the researcher team will have access to the records. However, your records may be reviewed for audit purposes by authorized University or other agents who will be bound by the same provisions of confidentiality.

\section{COMPENSATION \& COSTS}

You will receive $\$ 1.00$ for completing the questionnaire using a valid Invitation Code. There is no costs to participate in this study.

\section{RIGHT TO DECLINE OR WITHDRAW}

Your participation in this study is voluntary. You are free to participate in the study or withdraw your consent at any time during the study. Your withdrawal or lack of participation will not affect any benefits to which you are otherwise entitled. The investigator reserves the right to remove you without your consent at such time that they feel it is in the best interest.

\section{RESEARCHER CONTACT INFORMATION}

If you have any questions about the purpose, procedures, or any other issues relating to this research study you may contact Armando Falcon at DM 384A, 305-741-9977, afalc008@fiu.edu.

\section{IRB CONTACT INFORMATION}

If you would like to talk with someone about your rights of being a subject in this research study or about ethical issues with this research study, you may contact the FIU Office of Research Integrity by phone at 305-348-2494 or by email at ori@fiu.edu. 


\section{PARTICIPANT AGREEMENT}

Please make the following affirmations (Click on each statement to indicate agreement, then select NEXT):

$\square$ I have read the information in this consent form.

$\square$ I have had a chance to ask any questions I have about this study, and they have been answered to my satisfaction.

$\square$ I consent to the terms of this study and wish to participate. I understand that I may withdraw my consent at any time without penalty. 
Coworker - Questionnaire

Informed consent

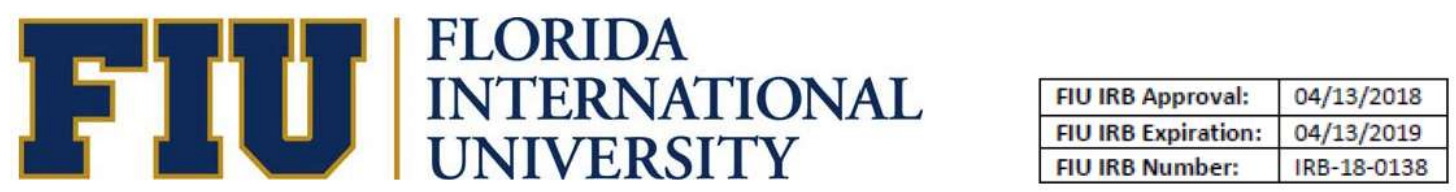

\section{ADULT ONLINE CONSENT TO PARTICIPATE IN A RESEARCH STUDY}

\section{Survey of Workplace Experiences}

\section{PURPOSE OF THE STUDY}

You are being asked to be in a research study. The purpose of this study is to leam more about the feelings and experiences of adult workers, and how those experiences are reflected in their on-the-job behaviors.

\section{NUMBER OF STUDY PARTICIPANTS}

Five hundred persons will be recruited through FIU's SONA System and another 500 persons will be recruited through Amazon's Mechanical Turk (MTurk) Platform. Further, each participant is asked to invite one or more coworkers to participate in the study. Thus, if you decide to participate, you will be one of approximately 2000 participants.

\section{DURATION OF THE STUDY}

Participation will require 10 minutes of your time. We ask that you complete the questionnaire during a single session.

\section{PROCEDURES}

If you agree to be in the study, we will ask you to do the following things:

1. Complete a questionnaire about your personal experiences at work and about the on-the-job behaviors of the coworker that invited you to participate in the study.

\section{RISKS AND/OR DISCOMFORTS}

The following risks/discomforts may be associated with participation in this study: 
1. There is the risk of coercion and/or feeling obligated to participate in the study, particularly if the coworker that invited you to participate has a higher organizational rank than you do.

2. You may experience psychological discomfort as a result of having to report the on-the-job behaviors of their coworkers.

3. You may expericence psychological discomfort when asked to recall your attitudes and experiences at work, particularly if you feel your current workplace situation is unfavorable.

If you find yourself experiencing psychological discomfort, you may contact FIU's Counseling and Psychological Services at 305-348-CAPS (-2277).

\section{BENEFITS}

There are no direct benefits to you as an individual. However, you may come to a better understanding of your workplace attitudes as a result of reflecting on your work experiences.

The information you provide will enable researchers to understand the current state of the workplace and develop targeted solutions to improve working conditions.

Improving working conditions benefits not only the workers themselves, but also the organizations they staff by enhancing organizational functioning. Indeed, society at large benefits from a more efficient workforce.

\section{ALTERNATIVES}

There are no known alternatives available to you other than not taking part in this study. However, any significant new findings developed during the course of the research which may relate to your willingness to continue participation will be provided to you.

\section{CONFIDENTIALITY}

The records of this study will be kept confidential and protected to the fullest extent provided by law. The person whom invited you to participate in this study--and whose workplace behaviors you will be asked about--will not know whether you have elected to 
participate. In other words, your participation in this study is fully confidential. Moreover, your identity and responses will never be shared with anyone outside the research team.

Moreover, in any sort of report we might publish, we will not include any information that will make it possible to identify a participant. Research records will be stored securely and only the researcher team will have access to the records. However, your records may be reviewed for audit purposes by authorized University agents who will be bound by the same provisions of confidentiality.

\section{COMPENSATION \& COSTS}

You will receive $\$ 1.00$ for completing the questionnaire using a valid Invitation Code.

There are no costs to participate in this study.

\section{RIGHT TO DECLINE OR WITHDRAW}

Your participation in this study is voluntary. You are free to participate in the study or withdraw your consent at any time during the study. Your withdrawal or lack of participation will not affect any benefits to which you are otherwise entitled. The researcher reserves the right to remove you without your consent at such time that they feel it is in the best interest.

\section{RESEARCHER CONTACT INFORMATION}

If you have any questions about the purpose, procedures, or any other issues relating to this research study you may contact Armando Falcon at DM 384A, 305-741-9977, afalc008@fiu.edu.

\section{IRB CONTACT INFORMATION}

If you would like to talk with someone about your rights of being a subject in this research study or about ethical issues with this research study, you may contact the FIU Office of Research Integrity by phone at 305-348-2494 or by email at ori@fiu.edu.

\section{PARTICIPANT AGREEMENT}

Please make the following affirmations (Click on each statement to indicate agreement, then select NEXT): 
I have read the information in this consent form.

I have had a chance to ask any questions I have about this study, and they have been answered to my satisfaction.

I consent to the terms of this study and wish to participate. I understand that I may withdraw my consent at any time without penalty.

Please enter your Invitation Code (This number is provided by the coworker whom invited you to participate in this study.)

\section{Browser Meta Info}

This question will not be displayed to the recipient.

Browser: Chrome

Version: 67.0.3396.87

Operating System: Windows NT 10.0

Screen Resolution: $1920 \times 1080$

Flash Version: -1

Java Support: 0

User Agent: Mozilla/5.0 (Windows NT 10.0; Win64; x64) AppleWebKit/537.36 (KHTML, like

Gecko) Chrome/67.0.3396.87 Safari/537.36

These page timer metrics will not be displayed to the recipient.

First Click: 0 seconds

Last Click: 0 seconds

Page Submit: 0 seconds

Click Count: 0 clicks

\section{Perceived Ethical Climate}

Consider your current workplace. Use the scale provided to indicate the extent to which you agree or disagree with each statement.

In this company...

$\begin{array}{cccc}\text { Strongly } & \text { Neither } & & \\ \text { Disagree } & \text { Disagree } & \begin{array}{c}\text { Agree nor } \\ \text { Disagree }\end{array} \quad \text { Agree } & \begin{array}{c}\text { Strongly } \\ \text { Agree }\end{array}\end{array}$


what is best for everyone in the company is the major consideration here.

the most important concern is the good of all the people in the company as a whole.

the major concern is always what is best for the other person.

people look out for each other's good.

people are expected to comply with the law and professional standards over and above other considerations.

the law or ethical code of their profession is the major consideration.

people are expected to strictly follow legal or professional standards.

the first consideration is whether a decision violates any law.

it is very important to follow the company's rules and procedures here.

everyone is expected to stick by company rules and procedures.

successful people go by the book.

people strictly obey the company policies.

people are expected to follow their own personal and moral beliefs.
O

O

0

$0 \quad 0$

0

0

0

$0 \quad 0$

$0 \quad 0$

$0 \quad 0$

$0 \quad 0$

$0 \quad 0$

$0 \quad 0$

$0 \quad 0$

O 0

$0 \quad 0$

0

O

O

$\mathrm{O}$

0

0

0

0

0

0

0

O

O
Neither

Strongly

Disagree

Disagree

Agree nor Disagree

Agree

Strongly

Agree

each person decides for themselves what is right and wrong.
$0 \quad 0$ 
tne most important concern is each person's own sense of right and wrong.

people are guided by their own personal ethics.

people protect their own interests above all else.

people are mostly out for themselves.

there is no room for one's own personal morals or ethics.

people are expected to do anything to further company's interests, regardless of the consequences.

people are concerned with the company's interests -to the exclusion of all else.

work is considered substandard only when it hurts the company's interests.

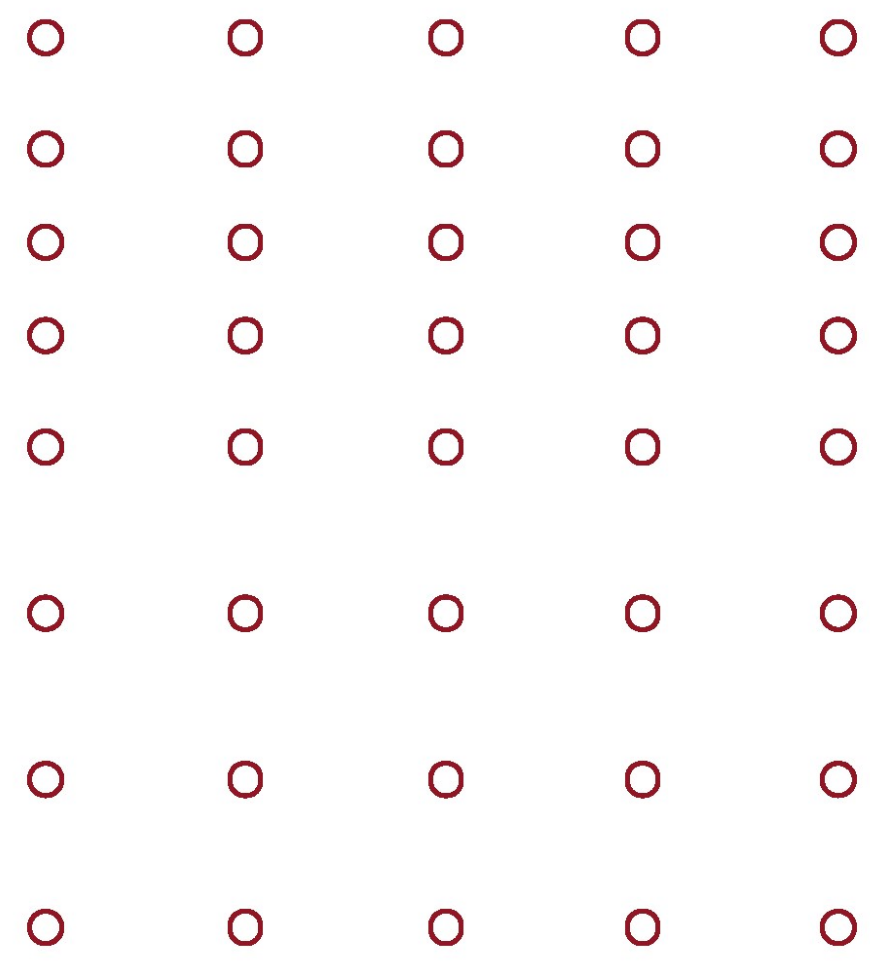

These page timer metrics will not be displayed to the recipient.

First Click: 0 seconds

Last Click: 0 seconds

Page Submit: 0 seconds

Click Count: 0 clicks

\section{Peer-rated Abusive Supervision}

During the past 30 days, how frequently did your coworker (i.e. the person whom invited you to participate in this study) experience following treatment from his or her supervisor?

$\begin{array}{lccccc} & \text { Never } & \text { Seldom } & \text { Occasionally } & \begin{array}{c}\text { Moderately } \\ \text { often }\end{array} & \text { Very often } \\ \text { Was Ridiculed } & \mathrm{O} & \mathrm{O} & \mathrm{O} & \mathrm{O} & \mathrm{O} \\ \begin{array}{l}\text { Was told their thoughts } \\ \text { or feelings are stupid. }\end{array} & \mathrm{O} & \mathrm{O} & \mathrm{O} & \mathrm{O} & \mathrm{O}\end{array}$




\begin{tabular}{|c|c|c|c|c|c|}
\hline & Never & Seldom & Occasionally & $\begin{array}{l}\text { Moderately } \\
\text { often }\end{array}$ & Very ofter \\
\hline $\begin{array}{l}\text { Gave them the silent } \\
\text { treatment. }\end{array}$ & 0 & 0 & 0 & 0 & 0 \\
\hline $\begin{array}{l}\text { Puts them down in } \\
\text { front of others. }\end{array}$ & 0 & 0 & 0 & 0 & 0 \\
\hline Invaded their privacy. & 0 & 0 & 0 & 0 & 0 \\
\hline $\begin{array}{l}\text { Reminded them of } \\
\text { their past mistakes } \\
\text { and failures. }\end{array}$ & 0 & 0 & 0 & 0 & 0 \\
\hline $\begin{array}{l}\text { Did not give them } \\
\text { credit for jobs requiring } \\
\text { a lot of effort. }\end{array}$ & 0 & 0 & 0 & 0 & 0 \\
\hline $\begin{array}{l}\text { Blamed them to save } \\
\text { himself/herself } \\
\text { embarrassment. }\end{array}$ & 0 & 0 & 0 & 0 & 0 \\
\hline Please select seldom. & 0 & 0 & 0 & 0 & $\mathrm{O}$ \\
\hline $\begin{array}{l}\text { Broke promises he/she } \\
\text { made. }\end{array}$ & 0 & 0 & 0 & 0 & 0 \\
\hline $\begin{array}{l}\text { Expressed anger at } \\
\text { them when he/she was } \\
\text { mad for another } \\
\text { reason. }\end{array}$ & 0 & $\mathrm{O}$ & 0 & 0 & 0 \\
\hline $\begin{array}{l}\text { Maked negative } \\
\text { comments about them } \\
\text { to others. }\end{array}$ & 0 & 0 & 0 & 0 & 0 \\
\hline Was rude to them. & 0 & 0 & 0 & 0 & $\mathrm{O}$ \\
\hline $\begin{array}{l}\text { Did not allow them to } \\
\text { interact with their } \\
\text { coworkers. }\end{array}$ & 0 & 0 & 0 & 0 & $\mathrm{O}$ \\
\hline $\begin{array}{l}\text { Told them they are } \\
\text { incompetent. }\end{array}$ & 0 & 0 & 0 & 0 & 0 \\
\hline Lied to them. & 0 & 0 & 0 & 0 & 0 \\
\hline
\end{tabular}

These page timer metrics will not be displayed to the recipient.

First Click: 0 seconds

Last Click: 0 seconds

Page Submit: 0 seconds

Click Count: 0 clicks

Peer-rated performance (task, ocbs, cwbs) 
Consider your coworker (i.e. the person whom invited you to participate in this study) and use the scale below to indicate how often he/she does the following things at work.

\begin{tabular}{|c|c|c|c|c|c|}
\hline & Never & Rarely & Sometimes & Quite often & $\begin{array}{l}\text { Extremely } \\
\text { often }\end{array}$ \\
\hline $\begin{array}{l}\text { Adequately completes } \\
\text { assigned duties. }\end{array}$ & 0 & 0 & 0 & 0 & 0 \\
\hline $\begin{array}{l}\text { Fulfills responsibilities } \\
\text { specified in job } \\
\text { description. }\end{array}$ & 0 & 0 & 0 & 0 & 0 \\
\hline $\begin{array}{l}\text { Performs tasks that } \\
\text { are expected of } \\
\mathrm{him} / \mathrm{her} \text {. }\end{array}$ & 0 & 0 & 0 & 0 & 0 \\
\hline $\begin{array}{l}\text { Meets formal } \\
\text { performance } \\
\text { requirements of the } \\
\text { job. }\end{array}$ & 0 & 0 & 0 & 0 & 0 \\
\hline $\begin{array}{l}\text { Helps new employees } \\
\text { get oriented to the job. }\end{array}$ & 0 & 0 & 0 & 0 & 0 \\
\hline $\begin{array}{l}\text { Offers suggestions to } \\
\text { improve how work is } \\
\text { done. }\end{array}$ & 0 & 0 & 0 & 0 & 0 \\
\hline $\begin{array}{l}\text { Offers suggestions for } \\
\text { improving the work } \\
\text { environment. }\end{array}$ & 0 & 0 & 0 & 0 & 0 \\
\hline $\begin{array}{l}\text { Volunteers for extra } \\
\text { work assignments. }\end{array}$ & 0 & 0 & 0 & 0 & 0 \\
\hline $\begin{array}{l}\text { Says good things } \\
\text { about your employer in } \\
\text { front of others. }\end{array}$ & 0 & 0 & 0 & 0 & 0 \\
\hline $\begin{array}{l}\text { Gives up meal and } \\
\text { other breaks to } \\
\text { complete work. }\end{array}$ & 0 & 0 & 0 & 0 & 0 \\
\hline $\begin{array}{l}\text { Lends a } \\
\text { compassionate ear } \\
\text { when someone had a } \\
\text { work problem. }\end{array}$ & 0 & 0 & 0 & 0 & 0 \\
\hline $\begin{array}{l}\text { Lends a } \\
\text { compassionate ear } \\
\text { when someone has a } \\
\text { personal problem. }\end{array}$ & 0 & 0 & 0 & 0 & 0 \\
\hline
\end{tabular}




Never Rarely Sometimes Quite often $\begin{gathered}\text { Extremely } \\ \text { often }\end{gathered}$

Changes vacation schedule, work days, or shifts to accommodate coworker's needs.

Helps a less capable co-worker lift a heavy box or other object.
0
0
O
O

0

Goes out of the way to give co-workers encouragement or express appreciation.

0

0

0

O

O

Defends a co-worker who is being "putdown" or spoken ill of by other co-workers or supervisors.

Takes property from work without permission.

Please select quite often.

Spends too much time fantasizing or daydreaming instead of working.

Takes an additional or longer break than is acceptable at your workplace.

Comes in late to work without permission.

O<smiles>O</smiles>

0

O

0

$\begin{array}{lllll}0 & 0 & 0 & 0 & 0\end{array}$

O

0

0

0

O

O

O

O

0

O

0

0

O

O

O

Litters his/her work environment.

O

$0 \quad 0$

0

0

0

0

0

O

0

0

0

0

Neglets to follow his/her boss's

instructions.

Intentionally works slower than he or she can work.

Discusses confidential information with an unauthorized person.

O

0

0

0

0

0

0

0

0

0

O

0

0 


\begin{tabular}{|c|c|c|c|c|c|}
\hline & Never & Rarely & Sometimes & Quite often & $\begin{array}{l}\text { Extremely } \\
\text { often }\end{array}$ \\
\hline $\begin{array}{l}\text { Uses an illegal drug or } \\
\text { consumes alcohol on } \\
\text { the job. }\end{array}$ & 0 & 0 & 0 & 0 & 0 \\
\hline $\begin{array}{l}\text { Puts llitle effort into his } \\
\text { or her work. }\end{array}$ & 0 & 0 & 0 & 0 & 0 \\
\hline $\begin{array}{l}\text { Drags out work in } \\
\text { order to get overtime. }\end{array}$ & 0 & 0 & 0 & 0 & 0 \\
\hline $\begin{array}{l}\text { Makes fun of someone } \\
\text { at work. }\end{array}$ & 0 & 0 & 0 & 0 & 0 \\
\hline $\begin{array}{l}\text { Says something hurtful } \\
\text { to someone at work. }\end{array}$ & 0 & 0 & 0 & 0 & 0 \\
\hline $\begin{array}{l}\text { Makes an ethnic, } \\
\text { religious, or racial } \\
\text { remark at work. }\end{array}$ & 0 & 0 & 0 & 0 & 0 \\
\hline $\begin{array}{l}\text { Curses at someone at } \\
\text { work. }\end{array}$ & 0 & 0 & 0 & 0 & 0 \\
\hline $\begin{array}{l}\text { Plays a mean prank on } \\
\text { someone at work. }\end{array}$ & 0 & 0 & 0 & 0 & 0 \\
\hline $\begin{array}{l}\text { Acts rudely toward } \\
\text { someone at work. }\end{array}$ & 0 & 0 & 0 & 0 & 0 \\
\hline $\begin{array}{l}\text { Publicly embarrasses } \\
\text { someone at work. }\end{array}$ & 0 & 0 & 0 & 0 & 0 \\
\hline
\end{tabular}

These page timer metrics will not be displayed to the recipient.

First Click: 0 seconds

Last Click: 0 seconds

Page Submit: 0 seconds

Click Count: 0 clicks

Demographics

\section{Participant Demographic Information}

IMPORTANT: The information collected in this section will allow the research team to better understand the overall characteristics of the persons who completed the study. Remember that the responses you provide in this section (like all of your other responses) will be kept private and confidential to the full extent of the law. 
Indicate your age. (in years)

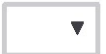

Indicate your gender.

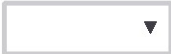

Indicate your race/ethnicity

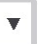

Indicate the highest degree you have earned.

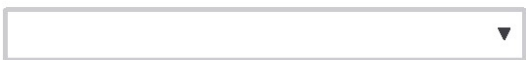

Which of the following BEST describes your current employment status?
Full-time
Part-time
Self-employed
Unemployed

During the course of carrying out your job, do you report to someone whom you would refer to as your "boss" and/or immediate supervisor?
$\mathrm{O}$ Yes
O No

In what State do you work? 


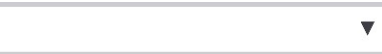

How many hours per week do you work? (on average)

\section{$\checkmark$}

How long have you worked for your current employer?

Months

Years

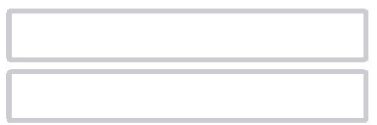

How long have you been at your current position/job classification?

Months

Years

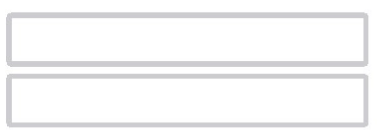

On average, how often do you work with your coworker (i.e. the person whom invited you to participate in this study).

Less than once per week

Once per week

Twice per week

Three or more times per week

Every day

How familiar are you with the job duties and responsibilities of your coworker(i.e. the person whom invited you to participate in this study)?

Extremely familiar

$O$ Very familiar

Moderately familiar

Slightly familiar 
Not familiar at all

Which of the following best describes the organizational relationship between yourself and your coworker (i.e. the person whom invited you to participate in this study)?

Supervisory relationship (he/she is above me, I report to him/her, he/she is my boss.)

Peer relationship (he/she is at the same organizational level as I am)

Subordinate relationship (he/she is below me, he/she reports to me, I am his/her boss)

Other (please describe)

Please describe the organizational relationship between yourself and your coworker (i.e. the person whom invited you to participate in this study).

Please select the industry/sector that BEST describes your current job.

Finally, does your job involve any of the following? (Check all that apply.)

Estimating the Quantifiable

Characteristics of

Products, Events, or Information

$\square$ Getting Information

Identifying Objects,

Actions, and Events

Inspecting Equipment,

Structures, or Material

Monitor Processes, Materials, or Surroundings

Analyzing Data or
Updating and Using Relevant Knowledge

Controlling Machines and

Processes

Documenting/Recording

Information

Drafting, Laying Out, and

Specifying Technical Devices,

Parts, and Equipment

Handling and Moving Objects

Interacting With Computers
Coordinating the Work and Activities of Others

Developing and Building Teams

Establishing and

Maintaining Interpersonal Relationships

Guiding, Directing, and Motivating Subordinates Interpreting the Meaning of Information for Others Monitoring and Controlling 


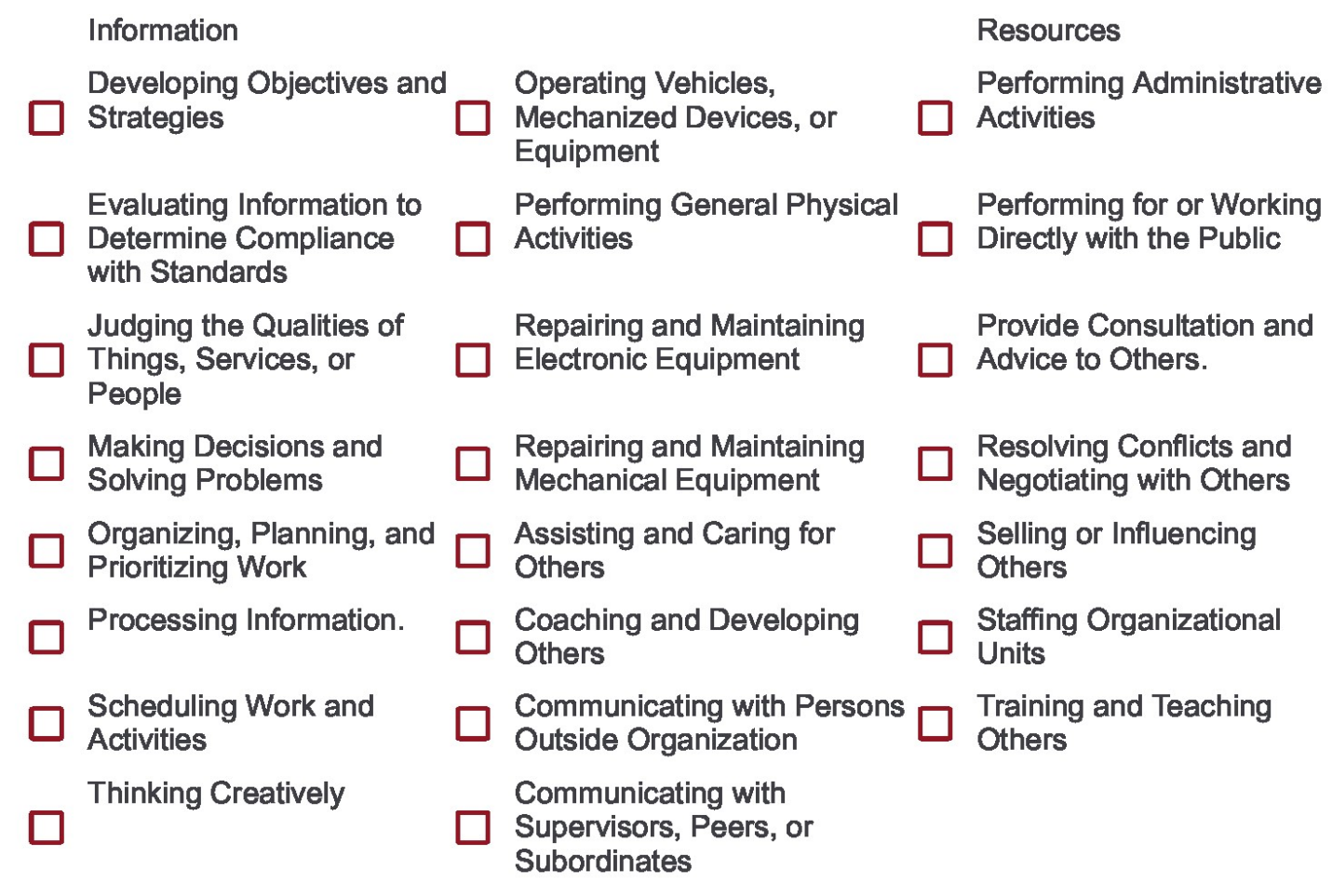

These page timer metrics will not be displayed to the recipient.

First Click: 0 seconds

Last Click: 0 seconds

Page Submit: 0 seconds

Click Count: 0 clicks 
VITA

\section{ARMANDO FALCON}

Born, Havana, Cuba

2010-2014

B.A., Psychology

Florida International University, Miami, FL

2014-2016

M.S., Psychology

Florida International University, Miami, FL

2016-2019

Doctoral Candidate, Psychology

Florida International University, Miami, FL

\section{PUBLICATIONS AND PRESENTATIONS}

Falcon, A., \& Viswesvaran, C. (2017, April). Comparison of counterproductive work behavior scores across single and multi-organization samples. Poster presented at the 32nd Annual Conference of the Society for Industrial and Organizational Psychology, Orlando, FL.

Falcon, A., \& Viswesvaran, C. (2017, April). Reliability generalization of Bennett and Robinson's workplace deviance scale. Poster presented at the 32nd Annual Conference of the Society for Industrial and Organizational Psychology, Orlando, FL.

Falcon, A., Manapragada, A. (2015, May). The mediating role of job-related negative affect in the job control-physical symptoms relationship. Interactive paper presented at the eleventh International Conference on Occupational Stress and Health. Atlanta, GA

Lanz, J. J., Falcon, A., Bruk-Lee, V., Manapragada, A., \& Schantz, A. (2016, April). Examining the nomological network of resilience. Poster presented at the 86th annual convention of the Rocky Mountain Psychological Association, Denver, CO.

Manapragada, A., Lanz, J.J., Falcon, A., Schantz, A. D., \& Bruk-Lee, V. (2014, May). Breaking the silence: An examination of employee silence behavior. Poster presented at the 29th Annual Conference of the Society for Industrial and Organizational Psychology, Honolulu, HI.

Schantz, A. D., Falcon, A., Lanz, J., Manapragada, A., \& Bruk-Lee, V. (2015, April). Drug, alcohol, and tobacco use to cope with workplace conflict. In M. A. Clark \& L. M. Zimmerman (Chairs), Addictions, vices, and work, Oh my! Symposium conducted at the 30th Annual Conference of the Society for Industrial and Organizational Psychology, Philadelphia, PA. 